\title{
Ultrafast Strong Field Dynamics in Dielectrics
}

\author{
Annkatrin Madlen Sommer
}

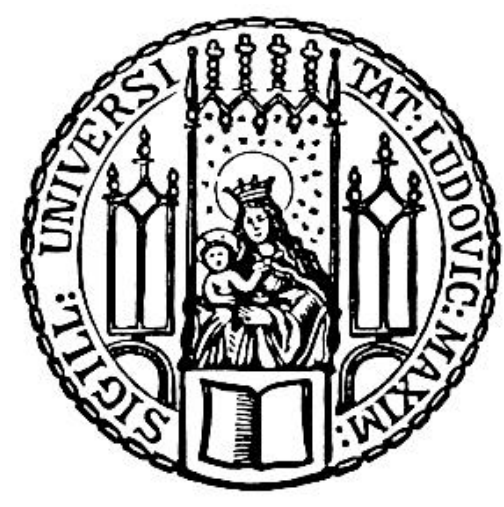

München 2015 



\title{
Ultrafast Strong Field Dynamics in Dielectrics
}

Annkatrin Madlen Sommer

\author{
Dissertation \\ an der Fakultät für Physik \\ der Ludwig-Maximilians-Universität \\ München
}

vorgelegt von

Annkatrin Madlen Sommer

aus Dachau

München, den 20.10.2015 
Erstgutachter: Prof. Dr. Ferenc Krausz

Zweitgutachter: Prof. Dr. Alexander Holleitner

Tag der mündlichen Prüfung: 02.12.2015 


\section{Contents}

$\begin{array}{llr}1 & \text { Introduction } & 1\end{array}$

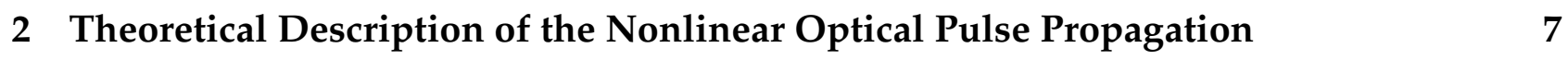

2.1 Self-Phase Modulation . . . . . . . . . . . . . . . . . . . . 10

2.2 Self-Focusing . . . . . . . . . . . . . . . . . . . . . . . 12

2.3 Self-Steepening . . . . . . . . . . . . . . . . . . . . . 13

2.4 Frequency Tripling . . . . . . . . . . . . . . . . . . . . . . . . . 15

2.5 Intrapulse Raman-Scattering . . . . . . . . . . . . . . . . . . . . . 16

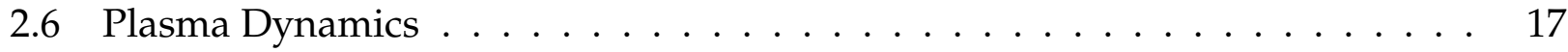

2.6 .1 The Contribution of Free Electrons to the Refractive Index . . . . . 17

2.6.2 The Nonresonant Contribution of Bound Electrons to the Refractive Index . . . . . . . . . . . . . . . . . . . . . 19

2.6 .3 The Polarization in a Plasma $\ldots \ldots \ldots$. . . . . . . . . . . . . 19

3 Time Integrated Investigation of the Nonlinear Kerr Coefficient 21

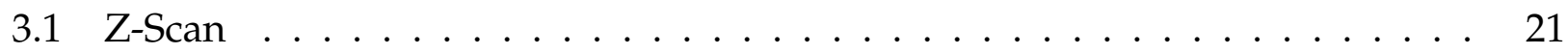

3.2 Intensity Dependent Reflectivity $\ldots \ldots \ldots \ldots \ldots \ldots \ldots$

\begin{tabular}{|lll}
4 & Attosecond Transient Absorption Spectroscopy & 29
\end{tabular}

5 Strong Field Modification of the Reflectivity 33

\begin{tabular}{|ll|}
6 & Attosecond Polarization Spectroscopy \\
\hline
\end{tabular}

6.1 Basic Concept . . . . . . . . . . . . . . . . . . . . . . . 38

6.2 Experimental Implementation $\ldots \ldots \ldots . \ldots \ldots 4 . \ldots \ldots$

6.3 Verification in the Gas Phase . . . . . . . . . . . . . . . . . . . . . . . . . 43

6.4 Nonlinear Interaction of few-cycle VIS/NIR Pulses with Solids. . . . . . . . . 50 
6.4 .1 The Nonlinear Refractive Index Change . . . . . . . . . . . . . . 50

6.4.2 Comparison of the Nonlinear Refractive Index of Different Dielectrics 56

6.4 .3 The Nonlinear Polarization Response . . . . . . . . . . . . . . . . 60

6.4 .4 Energy Transfer Dynamics . . . . . . . . . . . . . . . . . . . . . . . 63

6.4.5 Energy Consumption of a Hypothetical Ultrafast Optical Transistor . 70

6.4.6 Experimental Verification of the Derived Concept to Access the Nonlinear Polarization $\ldots \ldots \ldots \ldots \ldots \ldots \ldots \ldots \ldots$

6.5 Major Findings of APS $\ldots \ldots \ldots \ldots \ldots \ldots$

\begin{tabular}{lll}
\hline 7 & Outlook & 77
\end{tabular}

$7.1 \quad$ Wave Form Synthesis $\ldots \ldots \ldots$. . . . . . . . . . . . . 77

7.2 Investigation of Semiconductor Materials with APS $\ldots \ldots \ldots$. . . . . . 82

7.3 Extension of APS to Broadband Probe Fields . . . . . . . . . . . . . 82

$\begin{array}{llr}8 \text { Conclusion } & 85\end{array}$

\begin{tabular}{llr}
\hline & Appendix & 89
\end{tabular}

9.1 Laser System . . . . . . . . . . . . . . . . . . . . . . . . . . . . . . . . . . . . . . 89

9.1.1 Laser System Emitting Few Cycle Pulses with 500 $\mu$ J Pulse Energy _ . 89

9.1.2 Laser System Emitting Few-Cycle Pulses with $1 \mathrm{~mJ}$ Pulse Energy . . . 93

9.2 Chirped Dielectric Multilayer Mirrors … . . . . . . . . . . . . . . . . 93

9.3 Generation of Isolated Attosecond Pulses $\ldots \ldots \ldots$

9.4 Attosecond Streaking Spectroscopy . . . . . . . . . . . . . . . . . . . . . . . 102

9.5 Frequency Resolved Optical Gating ～. . . . . . . . . . . . . . . . . 106

9.6 Free Electron Trajectories in Neon Plasma . . . . . . . . . . . . . . . . . . . 112

9.7 Damage Threshold Measurement . . . . . . . . . . . . . . . . . . . . . . 112

9.8 The Impact of Linear Material Dispersion on the Nonlinearity . . . . . . . . 114

9.9 Technical Implementation of the Wave Form Synthesizer $\ldots \ldots \ldots$

$\begin{array}{ll}10 \text { Data Archiving } & 119\end{array}$

\begin{tabular}{lr}
\hline Bibliography & 123
\end{tabular}

\begin{tabular}{ll}
\hline Danksagung & 131
\end{tabular} 


\section{Abstract}

Due to the progress in laser technology in the last decades, stable and reliable sources for ultrashort light pulses are currently available [1]. These pulses contain only a couple of field cycles and possess a duration of a few femtoseconds at optical frequencies. If they were used in signal processing the clock frequency could be increased by more than a factor of 1000 compared to current electronic semiconductor technology [2]. The work presented in this thesis examines whether the ultrafast strong field dynamics in dielectrics can be exploited for such an application. For this purpose it is necessary to understand the physical origin of the different dynamics triggered in the light-matter interaction on femtossecond timescales.

If an electric field interacts with matter it excites oscillating dipoles which themselves emit a polarization wave. As long as the driving field is weak the dipoles follow the external force linearly. When the amplitude of the strong field becomes comparable to the interatomic forces the material response contains a nonlinear contribution. This nonlinear polarization includes all the characteristics of the nonlinear strong field interaction. It provides information about the response time of the system and the energy transfer dynamics throughout the interaction. The aim of this thesis is to extract the nonlinear polarization wave induced by optical few cycle fields in wide-gap solids.

To do so, the nonlinear interaction of intense sub-4-femtosecond $(f s)$ light pulses with insulators close to optical breakdown is investigated with different experimental approaches. In a first step the nonlinear Kerr coefficient is determined with time integrating techniques. The obtained result is $2-3$ times smaller than reference values reported in literature for the interaction with longer pulses [3]. To understand this deviation the induced material response has to be examined with a time resolved measurement. As the electron dynamics in solids evolve on sub femtosecond timescales, extreme ultraviolet (XUV) radiation with a few 100 attosecond (as) pulse duration provides the required temporal resolution. In the first experimental approach the modulation of the absorptivity of these attosecond pulses in the presence of the strong field is investigated. The examination reveals that the strong field triggers dynamics which can follow the driving force reversibly on sub-femtosecond timescales. In an additional nonlinear autocorrelation measurement, the highly nonlinear increase of the strong field reflectivity at intensities close to optical breakdown is observed.

To get a more comprehensive picture of the underlying dynamics which modify the XUV absorptivity and the amplitude of the reflected strong field, the experimental technique of 'Attosecond Polarization Spectroscopy' (APS) is developed. APS is an advancement of conventional pump-probe spectroscopy as it possesses attosecond resolution independent 
of the temporal extent of the probe pulse. It provides access to the complete nonlinear modification of the strong field throughout the light-matter interaction. As the experimental observables are analytically related to the nonlinear polarization, its amplitude and phase is directly accessible.

To verify the novel concept, APS is used to investigate the field induced ionization process and plasma dynamics in gas. The characteristic signature of the generated plasma can be detected in the modification of the optical material density. The nonlinear response of the medium is found to be dominated by the acceleration of the freed electrons in the field. The experimental results directly reveal that the excited electron wave packet oscillates on length scales of $2-3 \mathrm{~nm}$.

In the examination of the ultrafast strong field dynamics in dielectrics with APS, an increase of the optical material density in the presence of the strong field is observed. The modification is reversible on femtosecond timescales. Since APS provides access to the nonlinear polarization, the physical mechanisms underlying the nonlinear process can be revealed. The generation of virtual conduction band population [4] is identified as the source of the refractive index change. When the field strength increases at the beginning of the pulse, energy is transferred from the field to the material to move electrons away from their equilibrium positions. The projection of the laser dressed populated valence band states onto the empty conduction band states generates excited virtual carriers. As the field strength decreases the major part of the system returns to its undistorted initial state. The excited electrons decay via stimulated emission and energy flows back to the field. Only a small fraction of real carriers is excited into the conduction band throughout the interaction. Their negative contribution to the optical material density is not detectable in the experiment. The generated real conduction band population does not return to the valence band in the presence of the laser pulse. The carriers cannot return their excitation energy back to the field and therefore determine the energy irreversibly dissipated in the interaction.

The amount of energy transferred in the interaction influences the response time of the electronic system to the driving force. As absorption increases with the amplitude of the external field, the material response is decelerated with growing field strength. At intensities close to optical breakdown an upper limit of $<100$ as is extracted for the electronic response time of the system.

The maximum number of virtual carriers generated by the strong field scales with the square of its intensity as predicted by the Kerr effect. Since the presence of virtual conduction band population increases the optical density of the medium, the amplitude of the nonlinear refractive index change at the peak of the field envelope also follows the Kerr prediction. It grows linearly with intensity without any sign of saturation up to critical field strength. The temporal evolution of the refractive index change throughout the interaction cannot be described exclusively by the Kerr effect. Its sophisticated time structure is caused by the interplay between nonlinearity and dispersion for ultrashort broadband light pulses.

The manipulable refractive index could be used as a signal in future ultrafast signal processing. The real charges generated in the interaction are excited via multiphoton absorption. For wide-gap gap solids and optical fields this means that their number increases with the 
sixth power of the field intensity. The different scaling of the generation mechanism of virtual and real carriers with intensity can be used to maximize the ratio between the induced change in optical density and the energy dissipated in the nonlinear interaction. It can be exploited to optimize the parameters of a future optical switch operating at clock rates above 100 terahertz $(\mathrm{THz})$. By reducing the signal to the smallest detectable level, the energy density dissipated in one switching cycle can be reduced several orders of magnitude below the characteristic parameters of state-of-the-art gigahertz $(\mathrm{GHz})$ electronic transistors [5].

The results obtained from the APS experiments show that the optical material density of dielectrics can be reversibly manipulated with ultrashort strong fields on femtosecond timescales. The medium reacts fast enough to follow fields oscillating at petahertz $(\mathrm{PHz})$ frequencies. The amount of energy dissipated in the interaction is effectively tunable with the intensity of the driving field. These findings prove that the modulation of the optical properties of dielectrics with few femtosecond strong fields is a valid approach for future ultrafast signal processing. 


\section{Zusammenfassung}

Durch den Fortschritt, der in der Lasertechnologie in den letzten Jahrzehnten gemacht wurde, stehen heute zuverlässige und stabile Systeme zur Erzeugung intensiver ultrakurzer Lichtpulse zur Verfügung [1]. Diese Pulse enthalten nur einige Feldzyklen und sind im Sichtbaren wenige Femtosekunden lang. Wenn es möglich wäre, sie in der Signalverarbeitung zu nutzen, könnte die Schaltfrequenz um einen Faktor 1000 im Vergleich zu herkömmlichen elektronischen Transistoren gesteigert werden [2]. Das Ziel dieser Doktorarbeit ist es herauszufinden, ob Starkfeldeffekte, die ultrakurze Lichtpulse in Dielektrika induzieren, in der Signalverarbeitung genutzt werden können. Dazu ist es notwendig, die physikalischen Prozesse, die in der Wechselwirkung auf Femtosekunden Zeitskalen angeregten werden zu verstehen.

Ein elektrisches Feld erzeugt im Medium oszillierende Dipole, die selbst wieder eine Polarisationswelle emittieren. Solange das externe Feld schwach im Vergleich zu den interatomar wirkenden Kräften ist, können die induzierten Dipole ihm linear folgen. Starke Felder verändern die Energiestruktur des Materials so sehr, dass die abgestrahlte Polarisationsantwort einen nichtlinearen Beitrag enthält. Dieser nichtlineare Anteil der Materialantwort beinhaltet die gesamte Information über die nichtlineare Dynamik im Medium. Durch ihn wird die zeitliche Entwicklung des Energietransfers während der Wechselwirkung und die Antwortzeit des Systems zugänglich. In dieser Arbeit soll die nichtlineare Polarisation, die in der Wechselwirkung von ultrakurzen Lichtpulsen mit Dielektrika abgestrahlt wird, experimentell zugänglich gemacht werden.

Dazu werden die Starkfeldeffekte, die intensive Lichtpulse mit weniger als 4 Femtosekunden Pulsdauer in Isolatoren anregen mit verschiedenen experimentellen Techniken untersucht. Zunächst wird der nichtlineare Kerr Koeffizient mit Messmethoden bestimmt, die zeitlich über die gesamten Dauer der Licht-Materie Wechselwirkung mitteln. Um zu verstehen, warum das extrahierte Ergebnis deutlich unterhalb der Referenzwerte für längere Pulse liegt, [3], muss die Wechselwirkung zeitaufgelöst untersucht werden. Da sich Elektronendynamik in Festkörpern auf Atto- bzw. Femtosekunden Skalen abspielt, liefert extrem ultraviolette (XUV) Strahlung mit wenigen 100 Attosekunden Pulsdauer, die gewünschte Zeitauflösung. Es stellt sich heraus, dass die Wechselwirkung eines starken Femtosekunden Pulses mit dem Dielektrikum die Absorption des Attosekunden Pulses im Material beeinflusst. Die experimentellen Resultate ergeben, dass das starke Feld Dynamik im Material triggert, die dem hochfrequenten Feld reversibel folgt. Zusätzlich zeigt eine nichtlineare

Autokorrelation, dass die Reflektivität des intensiven Feldes nahe der Zerstörschwelle des Materials stark nichtlinear mit der Feldintensität zunimmt. 
Die Entwicklung der 'Attosekunden Polarisations-Spektroskopie' (APS), ermöglicht es, ein ganzheitlicheres Bild der Licht-Materie Wechselwirkung zu erhalten. APS ist eine Weiterentwicklung konventioneller Anrege-Abfrage Spektroskopie: die Zeitauflösung ist nicht mehr durch die Feldeinhüllende des Abfragepulses bestimmt, sondern liegt unabhängig davon bei wenigen 100 Attosekunden. Mit der experimentellen Technik ist der Einfluss der nichtlineare Materialantwort auf das starke Feld direkt messbar. Da die experimentell zugänglichen Größen analytisch mit der nichtlinearen Polarisation verknüpft sind, ist ihre komplette Amplituden- und Phaseninformation direkt verfügbar.

Zunächst wird das neue Konzept in der Gasphase verifiziert: die durch ein starkes Feld induzierte Ionisation und Plasmadynamik wird mit APS untersucht. Es ist möglich, die Abnahme des Brechungsindex durch die Erzeugung freier Ladungsträger nachzuweisen. Die nichtlineare Polarisation wird vom Beitrag der freien Ladungsträger dominiert. Aus den experimentellen Ergebnissen kann direkt bestimmt werden, dass das angeregte Elektronenwellenpakets auf Längenskalen von $2-3 \mathrm{~nm}$ hin und her oszilliert.

Die Untersuchung der nichtlinearen Wechselwirkung von ultrakurzen Lichtpulsen mit Dielektrika zeigt, dass die optische Dichte des Materials durch das starke Feld erhöht wird. Die Modifizierung verschwindet mit dem Puls. Da mit der APS Messtechnik die nichtlinearen Polarisation direkt zugänglich ist, können Rückschlüsse auf die physikalischen Mechanismen, die dem nichtlinearen Prozess zugrunde liegen gezogen werden. Es ist möglich, die Existenz virtueller Ladungsträger als Ursache für die Erhöhung der optischen Materialdichte im starken Feld zu identifizieren. Mit steigender Feldstärke zu Beginn des Pulses werden die Elektronen im Material immer weiter von ihrer Gleichgewichtslage weg bewegt. Dazu fließt Energie vom Feld zum Medium. Durch die Projektion der besetzten Valenzband Zustände auf die leeren Leitungsband Zustände wird eine virtuelle Leitungsbandpopulation erzeugt. Wenn die Feldstärke abnimmt, kehren die Ladungsträger größtenteils in ihre Ursprungszustände zurück und die dissipierte Energie fließt an das Feld zurück. Während der Wechselwirkung wird nur ein geringer Prozentsatz an realen Ladungsträgern im Leitungsband erzeugt. Ihr negativer Beitrag zur optischen Materialdichte ist in dem verwendeten Messaufbau nicht detektierbar. Die reale Leitungsbandpopulation bleibt während der Wechselwirkung angeregt und zerfällt erst nachdem der Puls verschwunden ist. Damit kann die Anregungsenergie nicht an das Feld zurückgegeben werden. Die Anzahl an real erzeugten Leitungsbandelektronen bestimmt also die Energie, die in der Licht-Materie Wechselwirkung dissipiert wird.

Die Antwortzeit des elektronischen Systems auf die externe Anregung hängt von der Menge an Energie, die zwischen Feld und Medium transferiert wird ab. Da Absorption mit steigender Feldstärke zunimmt, wächst auch die Zeitspanne, nach der das Material dem Feld folgt, mit der Amplitude des externen Feldes an. Bei Feldstärken kurz unterhalb der Zerstörschwelle des Materials reagiert das elektronische System innerhalb von $<100$ as.

Die Anzahl an virtuellen Ladungen wächst gemäß dem Kerr Effekt proportional zum Quadrat der Intensität des einfallenden Feldes. Da die Erzeugung virtueller Ladungsträger die optische Dichte des Materials erhöht, folgt auch die Brechungsindexänderung, die das starke Feld am Maximum seiner Einhüllenden erzeugt der Kerr Vorhersage. Sie wächst linear mit der Intensität des Feldes. Auch kurz unterhalb der Zerstörschwelle, ist keine Sättigung 
oder Abnahme der Nichtlinearität zu erkennen. Das zeitliche Profil der Brechungsindexänderung während des Pulses lässt sich hingegen nicht allein mit dem Kerr Effekt erklären. Es entsteht bei breitbandigen Femtosekunden Pulsen aus dem Zusammenwirken von Nichtlinearität und linearen Propagationseffekten.

Da die optische Dichte des Mediums mit ultrakurzen Pulsen reversibel moduliert werden kann, eignet sie sich als manipulierbares Signal in einem zukünftigen optischen Hochfrequenz Transistor

Die realen Ladungsträger werden via Multiphotonenabsorption ins Leitungsband angeregt. Damit skaliert ihre Anregungsrate deutlich nichtlinearer mit der Amplitude des starken Feldes als bei den virtuellen Ladungen. Das heißt, dass das Verhältnis zwischen induzierter Änderung der optischen Materialdichte und dissipierter Energie schnell invers zu der Intensität des Feldes ansteigt. Diese Abhängigkeit kann genutzt werden, um den Energieverbrauch eines zukünftigen optischen Transistor mit Taktraten über 100 Terahertz zu optimieren. Wenn mit dem kleinstmöglichen detektierbaren Signal gearbeitet wird, kann die in der Interaktion dissipierte Energiedichte um mehrere Größenordnungen unter die Kennwerte konventioneller $\mathrm{GHz}$ MOSFETs gesenkt werden [5].

Die Ergebnisse des APS Experiments zeigen, dass die optische Dichte von Dielektrika mit ultrakurzen intensiven Lichtpulsen reversibel manipuliert werden kann. Das System reagiert schnell genug, um Feldern mit Petahertz Frequenz zu folgen. Die in der Interaktion dissipierte Energie kann mit der Intensität des externen Feldes optimiert werden. Daher ist der Ansatz, Dielektrika mit ultrakurzen starken Feldern zu modulieren geeignet, um optischer hochfrequenz Transistoren zu realisieren. 


\section{List of Publications and Conference Contributions}

- A. Sommer, E. M. Bothschafter, S. A. Sato, C. Jakubeit, T. Latka, O. Razskazovskaya, H. Fattahi, M. Jobst, W. Schweinberger, V. Shirvanyan, V. S. Yakovlev, R. Kienberger, K. Yabana, N. Karpowicz, M. Schultze, F. Krausz," Attosecond nonlinear polarization and energy transfer in dielectrics.", submitted.

- M. Schultze, E.M. Bothschafter, A. Sommer, S. Holzner, W. Schweinberger, M. Fiess, M. Hofstetter, R. Kienberger, V. Apalkov, V. S. Yakovlev, M. I. Stockman, F. Krausz, "Controlling dielectrics with the electric field of light.", Nature 493, 758 (2013).

- W. Schweinberger, A. Sommer, E. M. Bothschafter, J. Li, F. Krausz, R. Kienberger, M. Schultze,"Waveform-controlled near-single-cycle milli-joule laser pulses generate sub-10nm extreme ultraviolet continua", Optics Letters 37(17), 3573-3575 (2012).

- A. Sommer, E. M. Bothschafter, S. A. Sato, C. Jakubeit, T. Latka, O. Razskazovskaya, H. Fattahi, M. Jobst, W. Schweinberger, V. Shirvanyan, V. S. Yakovlev, R. Kienberger, K. Yabana, N. Karpowicz, M. Schultze, F. Krausz,"Attosecond Spectroscopy of Nonlinear Polarization Dynamics.", Conference on Ultrafast Optics, Beijing (China) 2015.

- M. Schultze, E. M. Bothschafter, A. Sommer, S. Holzner, M. Fiess, M. Hofstetter, R. Kienberger, V. Apalkov, V. S. Yakovlev, M. I. Stockman, F. Krausz,"Strong-field-induced attosecond dynamics in $\mathrm{SiO}_{2} . "$, EPJ Web of Conferences 41, 02014 (2013). 


\section{Chapter 1}

\section{Introduction}

Scientific and technological progress in the last century provided access to powerful and cheap computing. This development strongly influenced the industrial structure and productivity. By enabling worldwide communication it modified social structures and procedures in everyday life. According to Gordon E. Moore's prediction in 1965 [6] this development will further proceed as the processing power of an integrated circuit is doubled every second year. This challenging goal can only be realized by steady increase of the amount of transistors in a microprocessor or rise of their clock frequency. One approach to achieve both aims is to decrease the size of the individual transistors. The invention of new fabrication techniques like UV (ultraviolet) lithography enables the manufacturing of transistors with dimensions below the diffraction limit of the UV light [7]. The microprocessor 'IBM Z13' released on 14th January 2015 includes $7 \cdot 10^{9}$ transistors each of $22 \mathrm{~nm}$ size which can be operated at a frequency of $5 \mathrm{GHz}[8]$. To even further decrease the spatial dimensions of the devices below $10 \mathrm{~nm}$ extreme ultraviolet light $(\lambda \approx 13 \mathrm{~nm})$ is utilized in the lithographic process [9]. Nevertheless, these elaborate manufacturing techniques are very expensive.

In the last 50 years technology has succeeded in downscaling the size of electronic structures into the nanoscale range and controlling their electronic properties at frequencies up to $100 \mathrm{GHz}[2,5,10-13]$. Further decrease of the transistor size is prevented by a new physical boundary: on atomic length scales quantum mechanical effects have to be considered. Field effect transistors used in modern information technology are fabricated from semiconductor materials. Silicon atoms have a spatial extent of approximately $0.3 \mathrm{~nm}$, so a distance of $5 \mathrm{~nm}$ can be filled with 17 atoms. Such a few atom system has to be treated in a quantum mechanical way. It is necessary to consider the non zero probability of electrons tunneling through the energetically forbidden regions between the individual transistors. This makes the performance of the electronic device unreliable and unstable [14].

In principle three basic types of solids, distinguishable by their characteristic electronic structure, exist: metals, semiconductors and insulators (Fig.[1.1]) [15]. In metals the Fermi level is located in the conduction band, which therefore is permanently populated with charge carriers. Under the influence of a weak external field these free charges flow in a current proportional to the amplitude of the driving force. Like this the buildup of a charge 
density gradient inside the material is avoided and the external field cannot penetrate into the medium. Due to the complex dynamics of this screening mechanism it is hard to predictably manipulate and control the electronic properties of metals and use them as switching devices in signal processing.

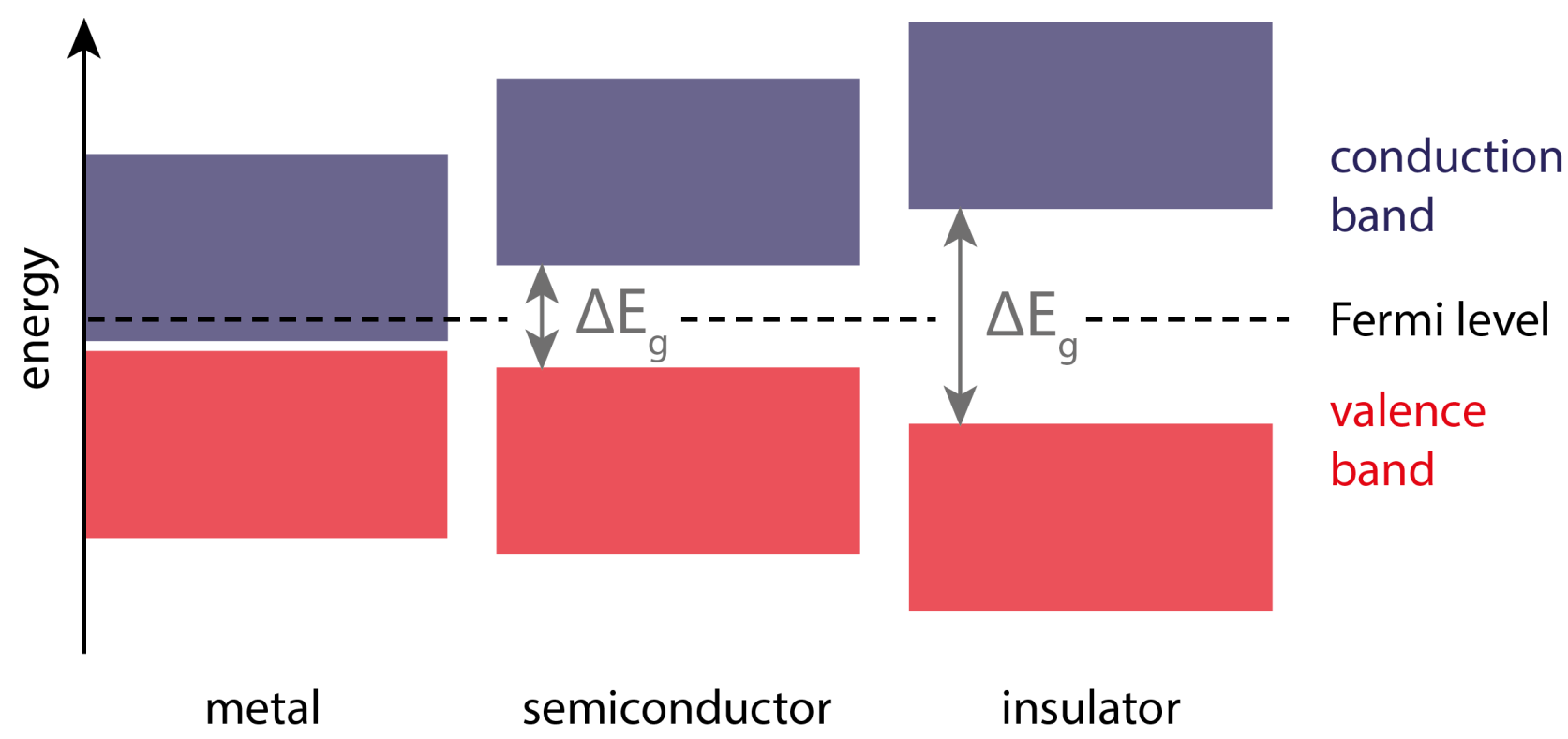

Figure 1.1: Schematic representation of the band structure of metals, semiconductors and dielectrics: In metals the Fermi level lies above the conduction band edge. In semiconductors and insulators the valence and conduction band are separated by an energetically forbidden gap $\Delta E_{g}$. Carriers can only be excited into the conduction band by an external force. The bandgap of semiconductors is smaller than for dielectrics.

Semiconductors and dielectrics are characterized by an energetically forbidden region between the valence and conduction band (Fig.(1.1)). In semiconductors this gap has a size of $0.1 \mathrm{eV}<\Delta E_{g}<4 \mathrm{eV}$. If an electron gains energy from an external source it can be excited across the gap. Then its wave function is described as a superposition of the valence band ground state and the excited state in the conduction band. The probability amplitude oscillates at a frequency of $0.02-1 \mathrm{PHz}$ between the two states. For insulators the band gap exceeds $\Delta E_{g}>4 \mathrm{eV}$ which corresponds to an oscillation period of less than $1 \mathrm{fs}$. Therefore dielectrics exhibit the fastest electronic dynamics [16-19].

The conduction band population in semiconductor structures can be manipulated with an external voltage. The induced transitions between conductive and non conductive state are currently exploited in signal processing. Thereby the clock rate is limited by the impedance of electrodes and interconnects. To overcome this boundary approaches for optical current injection and control have been developed [20-24]. Light fields with up to terahertz frequencies can be used to excite and direct conduction band carriers in semiconductor materials [25]. The field induced change of the electronic structure and population affects the optical properties of the medium. The nonlinear modification of the refractive index in the presence of a strong light pulse is exploited in concepts for all-optical switches [26]. A strong terahertz pump 
pulse is employed to trigger the nonlinearity which affects the propagation of a weak probe pulse on sub picosecond timescales [27, 28]. The modulation of the probe can be detected in an interferometric setup described i.e. by Lattes et al. [26].

Regarding dielectrics, only UV radiation or optical strong fields possess enough energy to excite electrons from the valence to the conduction band. Moreover a considerable amount of generated free charges in the conduction band is synonymous with irreversible material damage. For this reason dielectrics were for a long time considered unsuitable for signal processing applications. However recent research hints to the feasibility of controlling currents in insulators with intense optical few-cycle laser pulses [29,30]. For future application the energy dissipated in the light-matter interaction is an important and critical parameter. The nonlinear response of dielectrics is expected to contain a reversible contribution, which can be exploited for signal processing, and dynamics with a lifetime exceeding the duration of the driving field. This irreversible part determines the dissipated energy and has not been experimentally investigated in the strong field interaction of dielectrics with ultrashort pulses so far.

Ultrafast pulse technology quickly progressed in the last decades. With the decrease in pulse duration the achievable peak intensity grew [31]. Additionally the threshold for material breakdown is considerably increased in the interaction with ultrashort fields compared to longer pulses [32]. Titanium-Sapphire based laser systems (appendix 9.1.1) reliably emit intense optical few femtosecond pulses. If it were possible to use these ultrashort strong pulses to manipulate the electronic properties of dielectrics, optical switches with a clock rate of $>100 \mathrm{THz}$ could be realized.

To evaluate the feasibility of this approach two fundamental questions have to be answered:

- How fast does the material react to the external strong field and how quickly can it follow the driving wave?

- How big is the reversible contribution to the nonlinear material response compared to the irreversible part and is it possible to optimize the ratio between them for neardissipation-free optical signal processing?

The work presented in this thesis attempts to answer these questions by using different experimental approaches to investigate the strong field dynamics induced in dielectrics when illuminated with optical few cycle pulses. The principle proceeding is sketched in the following paragraph.

\section{A Descriptive Outline}

In insulators an external field excites electronic dipoles which themselves emit a polarization wave. If the amplitudes of the driving field is sufficiently strong, the generated dipoles cannot follow the external force linearly anymore. The emitted nonlinear polarization wave is the essential quantity characterizing the induced strong field dynamics. It provides the answers to the questions pointed out above. This thesis aims to experimentally access the 
nonlinear polarization excited by ultrashort pulses. The generation of these optical few cycle fields is presented in the appendix 9.1 and 9.2 .

For longer pulse durations the nonlinear polarization is a well explored quantity and described by perturbation theory. The basic theoretical concepts and phenomena emerging in the nonlinear light-matter interaction are summarized in section 2.

The perturbative description uses the concept of the intensity dependent refractive index. As no values of the nonlinear Kerr coefficient are available in literature for the interaction of few femtosecond pulses with dielectrics, it is determined experimentally with two time integrated techniques (section 3). The extracted results are considerably smaller than values reported for longer driving pulses [3]. To understand this discrepancy the induced nonlinear dynamics have to be investigated with temporal resolution.

Extreme ultraviolet (XUV) pulses with durations of a few 100 as are short enough to resolve the evolution of the electronic material properties. The attosecond pulses emerge in the nonlinear process of high harmonic generation explained in appendix 9.3 . The temporal characterization of the few cycle strong field is performed with attosecond streaking spectroscopy (appendix 9.4).

The first approach to temporally resolve the induced nonlinear dynamics is to investigate the strong field modification of the XUV absorptivity with attosecond resolution (section 44. The results of this transient absorption spectroscopy reveal dynamics which are related to the carrier field of the driving pulse and reversible on femtosecond timescales.

To examine the nonlinear scaling of the induced effect, the intensity dependent reflectivity of the strong field is explored in chapter 5. A highly nonlinear increase of the reflectivity with electric field strength is observed. In a nonlinear autocorrelation measurement the phenomena is found to be reversible on femtosecond timescales.

The two different experiments reveal a nonlinear field effect which vanishes with the driving field within femtoseconds. This result proves that it is principally possible to reversibly manipulate dielectrics with intense few cycle pulses. It demonstrates the attractiveness of this approach for signal processing. However many aspects of the fundamental questions raised above remain unanswered. Each experiment probes individual features of the strong field interaction and cannot deliver a complete picture of the nonlinear dynamics. The nonlinear polarization is only accessible via complex theoretical modeling with a low signal to noise ratio.

As described above the reflectivity of the few cycle pulse is found to increase highly nonlinear with intensity. In principle the complete information about the nonlinear dynamics is encoded in the strong field modification of the temporal structure of the reflected field. As the field transmitted through the material possesses complementary information, the technique of Attosecond Polarization Spectroscopy (APS) is developed to time resolve its modification in the nonlinear process (section 6). The theoretical concept derived in chapter 6.1 provides a direct analytic relation between the physical observables and the induced nonlinear polarization. In a first step APS is verified by detecting the decrease in optical material density due to plasma formation in the gas phase (section 6.3). Then it is employed 
to investigate the intensity dependent strong field dynamics in two different dielectrics with attosecond resolution (section 6.4). With APS the complete temporal structure of the induced nonlinear polarization, the electronic response time and energy transfer dynamics are accessible. The obtained results can answer the questions raised above regarding the nonlinear interaction of few femtosecond pulses with dielectrics. It is possible to benchmark the performance of a hypothetical ultrafast optical device for signal processing with respect to current semiconductor technology.

In section 7 the future approaches for further extension of the APS technique are described. The main ideas are to add another degree of freedom by flexible control of the temporal profile of the strong field. This is facilitated with a two channel waveform synthesizer (section 7.1. appendix 9.9). Furthermore, the current scheme will be extended to broadband probe fields which enable a more direct investigation of the induced dynamics.

\section{A Summary of the Most Important Results}

Throughout this thesis, the strong field dynamics induced in dielectrics by few femtosecond pulses in the visible to near infrared wavelength range are examined. An experimental technique which allows to investigate the excited nonlinear polarization with attosecond resolution is developed. The response time of the electronic system is found to depend on the energy absorbed in the light-matter interaction. At field strengths close to the damage threshold of the material dielectrics respond to the driving force within less than 100 as. The induced strong field dynamics are dominated by two physical mechanisms: The excitation of virtual [4] and real carriers. The generation of virtual population increases the optical density of the medium. As the carriers directly vanish with the driving field the nonlinear modification of the refractive index is completely reversible on sub-femtosecond timescales. It could be used as the signal in a future ultrafast all-optical switch. The maximum amount of generated virtual carriers follows intensity scaling predicted by the Kerr effect. The number of excited real carriers defines the energy dissipated in the interaction as the lifetime of the charges exceeds the duration of the driving pulse. Since they are generated by multiphoton absorption their excitation probability grows more rapidly with the strength of the driving field than for virtual carriers. Therefore it is possible to optimize the ratio between induced signal and dissipated energy for future near dissipation-free ultrafast optical signal processing. 


\section{Chapter 2}

\section{Theoretical Description of the Nonlinear Optical Pulse Propagation}

An electric field $E(\vec{r}, t)$ interacts with the charged properties of matter. As electrons possess considerably less mass than the ionic cores, their dynamics excited by the driving field evolve on shorter timescales than the induced lattice oscillation.

The external field polarizes the material, inducing oscillating dipoles which themselves emit radiation. Therefore the propagation of the light inside the medium takes the form of a polariton, consisting of the electric field $E(\vec{r}, t)$ coupled to the polarization wave $P(\vec{r}, t)$. The propagation and interaction of $E(\vec{r}, t)$ and $P(\vec{r}, t)$ in the absence of free carriers and charges is described by the nonlinear wave equation [33]

$$
-\nabla^{2} E(\vec{r}, t)+\frac{1}{c^{2}} \frac{\partial^{2} E(\vec{r}, t)}{\partial t^{2}}=-\frac{1}{c^{2} \epsilon_{0}} \frac{\partial^{2} P(\vec{r}, t)}{\partial t^{2}}
$$

$\mathrm{c}$ is the speed of light in vacuum and $\epsilon_{0}$ the vacuum permittivity. Eqn.(2.1) uses the MKS unit system. This convention will be employed throughout the entire thesis. If the amplitude of the external field becomes sufficiently large ( $\geq 1 \frac{\mathrm{V}}{\mathrm{O}}$ in dielectrics) the dipoles cannot follow the driving field linearly anymore. This results in a nonlinear contribution $P^{N L}(\vec{r}, t)$ to the polarization response [34].

$$
P(\vec{r}, t)=P^{L}(\vec{r}, t)+P^{N L}(\vec{r}, t)
$$

$P^{L}(\vec{r}, t)$ represents the linear material response and accounts for the linear dispersion the different frequency components experience during propagation [33].

$$
P^{L}(\vec{r}, t)=\epsilon_{0} \chi^{(1)}(\vec{r}, t) E(\vec{r}, t)
$$

$\chi^{(1)}(\vec{r}, t)$ is the lowest order susceptibility. In general $\chi^{(l)}(\vec{r}, t)$ are tensors of the order $l+1$ and depend on the frequency of the applied field. For reasons of lucidity $\chi^{(l)}$ will be 
treated as scalars in the following and the material response is assumed to be instantaneous.

If the amplitude of the nonlinear contribution to the polarization is sufficiently small, it can be considered as a small perturbation of the system and approximated in a Taylor expansion [33].

$$
P^{N L}(\vec{r}, t)=\epsilon_{0} \chi^{(2)} E(\vec{r}, t)^{2}+\epsilon_{0} \chi^{(3)} E(\vec{r}, t)^{3}+\ldots
$$

The susceptibility and the first order material permittivity $\epsilon^{(1)}$ are connected by $\epsilon^{(1)}=1+$ $\chi^{(1)}$. Additionally $\epsilon^{(1)}$ is related to the linear refractive index $n_{0}^{2}=\epsilon^{(1)}$. Under consideration of Eqn.2.1] and this dependency Eqn.2.2) can be written as [33]

$$
-\nabla^{2} E(\vec{r}, t)+\frac{\epsilon^{(1)}}{c^{2}} \frac{\partial^{2} E(\vec{r}, t)}{\partial t^{2}}=-\frac{1}{c^{2} \epsilon_{0}} \frac{\partial^{2} P^{N L}(\vec{r}, t)}{\partial t^{2}}
$$

Fourier transformation of Eqn.2.5) into the frequency domain results in a differential equations for every frequency component $\widetilde{E}_{\omega}(\vec{r})$. The individual equations are coupled by the nonlinear source term $\widetilde{P}_{\omega}^{N L}(\vec{r})$ which involves all frequencies as it depends on the complete electric field.

$$
\nabla^{2} \widetilde{E}_{\omega}(\vec{r})+\frac{\epsilon^{(1)} \omega^{2}}{c^{2}} \widetilde{E}_{\omega}(\vec{r})=-\frac{\omega^{2}}{c^{2} \epsilon_{0}} \widetilde{P}_{\omega}^{N L}(\vec{r})
$$

with

$$
\begin{aligned}
E(\vec{r}, t) & =\frac{1}{2 \pi} \int \widetilde{E}_{\omega}(\vec{r}) \exp (i \omega t) d \omega \\
P^{N L}(\vec{r}, t) & =\frac{1}{2 \pi} \int \widetilde{P}_{\omega}^{N L}(\vec{r}) \exp (i \omega t) d \omega .
\end{aligned}
$$

This thesis focuses on the examination of the nonlinear interaction of ultrashort light pulses with matter. Particularly for this case Brabec and Krausz [35] have further elaborated Eqn.(2.6). The derived result is a generalized form of the nonlinear Schrödinger equation and describes a broad variety of nonlinear optical effects. To understand their physical origin it is very instructive to study the analysis of Brabec and Krausz in more detail. In the following the fundamental assumptions and the most important intermediate steps leading to the generalized nonlinear Schrödinger equation are presented.

Eqn.(2.6) can be simplified with the slowly-evolving-amplitude-approximation [33]. It implicates that the amplitude $A(\vec{r}, t)$ of the incident electric field $E(\vec{r}, t)=A(\vec{r}, t) \exp (-i(k z-$ $\omega t))+c c$. does not vary significantly within one wavelength. For $E(\vec{r}, t)$ propagating in $\mathrm{z}$ direction the following ansätze can be employed to solve Eqn.(2.6):

$$
E(\vec{r}, t)=A(\vec{r}, t) \exp \left(-i\left(k_{0} z-\omega_{0} t\right)\right)+c c
$$




$$
\begin{gathered}
A(\vec{r}, t)=\frac{1}{2 \pi} \int \widetilde{A}_{\omega}(\vec{r}) \exp (i \omega t) d \omega \\
P^{N L}(\vec{r}, t)=p^{N L}(\vec{r}, t) \exp \left(-i\left(k_{0} z-\omega_{0} t\right)\right)+c c . \\
p^{N L}(\vec{r}, t)=\frac{1}{2 \pi} \int \widetilde{p}_{\omega}^{N L}(\vec{r}) \exp (i \omega t) d \omega
\end{gathered}
$$

$\widetilde{A}_{\omega}(\vec{r})$ and $\widetilde{p}_{\omega}^{N L}(\vec{r})$ are the slowly varying amplitudes of the field and the nonlinear polarization wave in the frequency domain. $\omega_{0}$ is the center frequency and $k_{0}=\frac{2 \pi}{\lambda_{0}}$ the wavevector at the fundamental wavelength $\lambda_{0}$. Due to the slowly-evolving-amplitudeapproximation $\frac{\partial^{2} \widetilde{A}_{\omega}(\vec{r})}{\partial z^{2}} \approx 0$ in Eqn. 2.6) [1]. Higher order dispersion terms are considered with an expansion of the wavevector $k(\omega)$ into a Taylor series

$$
k(\omega)=k_{0}+k_{1}\left(\omega-\omega_{0}\right)+\underbrace{\frac{1}{2} k_{2}\left(\omega-\omega_{0}\right)^{2}+\ldots}_{\widetilde{D}_{\omega}}
$$

where $\widetilde{D}_{\omega}=\sum \frac{1}{l !} k_{l}\left(\omega-\omega_{0}\right)^{l}$ for $l \geq 2$.

$$
\begin{gathered}
k_{1}=\left[\frac{d k}{d \omega}\right]_{\omega=\omega_{0}}=\frac{1}{c}\left[n(\omega)+\omega \frac{d n(\omega)}{d \omega}\right]_{\omega=\omega_{0}}=\frac{1}{v_{g}\left(\omega_{0}\right)} \\
k_{2}=\left[\frac{d^{2} k}{d \omega^{2}}\right]_{\omega=\omega_{0}}=\frac{d}{d \omega}\left[\frac{1}{v_{g}\left(\omega_{0}\right)}\right]_{\omega=\omega_{0}}=\left[-\frac{1}{v_{g}^{2}\left(\omega_{0}\right)} \frac{d v_{g}}{d \omega}\right]_{\omega=\omega_{0}}
\end{gathered}
$$

$n(\omega)$ is the refractive index of the medium. The linear term $k_{1}$ in the expansion stands for the inverse group velocity of the pulse $v_{g} . k_{2}$ and the higher order terms represent the group velocity dispersion.

Under consideration of the slowly-evolving-amplitude-approximation (Eqn.(2.9), Eqn.(2.11) and the dispersion treatment presented above, Eqn. 2.6 is Fourier transformed back into time domaine. Subsequently a retarded time frame moving with the light pulse is introduced

$$
z=z^{\prime} \quad \tau=t-k_{1} z
$$

This leads to the following result [1], [33]

$$
\left[\Delta_{\perp}+2 i k_{0} \frac{\partial}{\partial z^{\prime}}\left(1+\frac{i k_{1}}{k_{0}} \frac{\partial}{\partial \tau}\right)+2 k_{0} D\left(1+\frac{i k_{1}}{k_{0}} \frac{\partial}{\partial \tau}\right)\right] A(\vec{r}, \tau)=-\frac{\omega_{0}^{2}}{\epsilon_{0} c^{2}}\left(1+\frac{i}{\omega_{0}} \frac{\partial}{\partial \tau}\right)^{2} p^{N L}(\vec{r}, \tau)
$$


with the temporal representation of $\widetilde{D}_{\omega}, D=\sum \frac{1}{l !} k_{l}\left(i \frac{\partial}{\partial t}\right)^{l}$.

In order to identify the different physical mechanisms which the individual terms in Eqn. (2.17) represent it is instructive to restrict the perturbative expansion of the nonlinear source term to its lowest order contributions (Eqn.(2.4)). In the course of this thesis only centrosymmetric materials are considered in which the even order contributions to the nonlinearity vanish. Hence $p^{N L}(\vec{r}, t)$ is approximated with

$$
p^{N L}(\vec{r}, t)=3 \epsilon_{0} \chi^{(3)}|A(\vec{r}, t)|^{2} A(\vec{r}, t) .
$$

To get an intuitive understanding of Eqn.2.17) the correction factors $\frac{i k_{1}}{k_{0}} \frac{\partial}{\partial \tau}$ and $\frac{i}{\omega_{0}} \frac{\partial}{\partial \tau}$ are neglected in a first step and group velocity dispersion is restricted up to second order $k_{2}$ (Eqn.(2.13). With these approximations Eqn.(2.17) can be written as [33]

$$
\frac{\partial A(\vec{r}, \tau)}{\partial z^{\prime}}=\left[\frac{i}{2 k_{0}} \Delta_{\perp}-\frac{i k_{2}}{2} \frac{\partial^{2}}{\partial \tau^{2}}+\frac{3 i \omega_{0}}{2 n_{0} c} \chi^{(3)}|A(\vec{r}, \tau)|^{2}\right] A(\vec{r}, \tau)
$$

This representation of the nonlinear Schrödinger equation highlights that three different physical mechanisms influence the amplitude $A(\vec{r}, \tau)$ of the electric field on the left hand side of Eqn.(2.19): the first term on the right side represents diffraction as it modifies the extension of the wave in $\mathrm{x}$ and $\mathrm{y}$ direction. The second contribution containing $k_{2}$ alters the temporal profile of the amplitude due to group velocity dispersion. The last term on the right hand side is proportional to $|A(\vec{r}, \tau)|^{2}$ and represents an intensity dependent nonlinear contribution known as the optical Kerr effect [33].

\subsection{Self-Phase Modulation}

By analogy with the relation between the linear refractive index $n_{0}$, the first order permittivity $\epsilon^{(1)}$ and the susceptibility $\chi^{(1)}$

$$
n_{0}^{2}=\epsilon^{(1)}=1+\chi^{(1)}
$$

the intensity dependence of the material's polarization response can be included in the description of the refractive index.

$$
n(t)^{2}=1+\chi^{(1)}+3 \chi^{(3)}|A(\vec{r}, t)|^{2}+. .
$$

As only media with inversion symmetry are considered in the course of this thesis, even order contribution to the polarization are ignored.

Eqn.(2.21) can be written as

$$
n(t)=n_{0}+n_{2} I(\vec{r}, t)
$$


with the nonlinear Kerr coefficient $n_{2}=\frac{3 x^{(3)}}{n_{0}^{2} \epsilon_{0} c}$ and the pulse intensity $I(\vec{r}, t)=\frac{1}{2} n \epsilon_{0} c|A(\vec{r}, t)|^{2}$

This notation illustrates that the optical Kerr effect introduces an intensity dependent nonlinear phase contribution $\Phi^{N L}$

$$
\Phi^{N L}(t)=\omega_{0} \frac{z}{c} n_{2} I(t)
$$

and modifies the instantaneous frequency $\omega$ of the electric field [34].

$$
\delta \omega=-\frac{d \Phi^{N L}}{d t}
$$

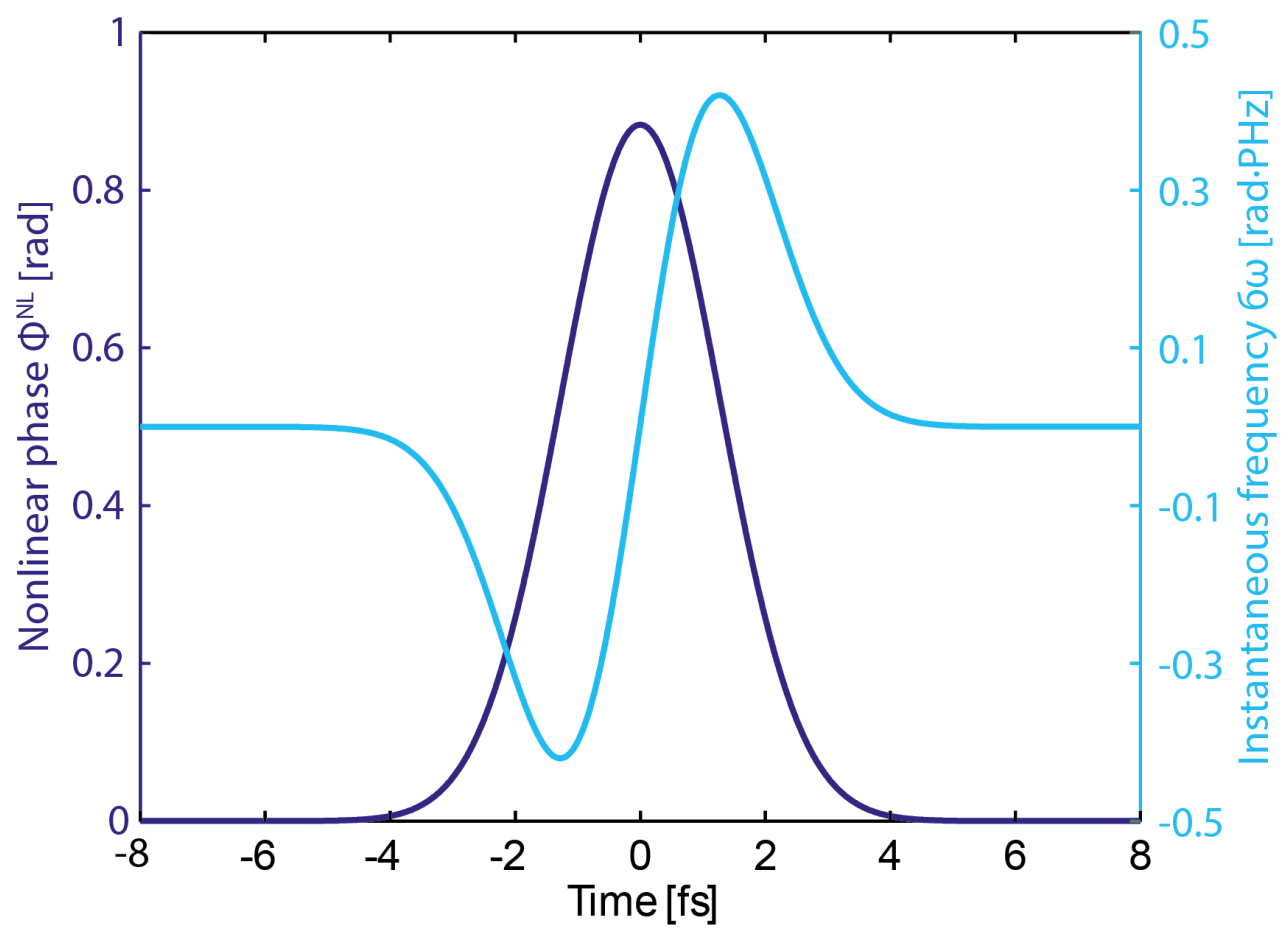

Figure 2.1: In a medium with positive $n_{2}$ a Gaussian pulse generates the nonlinear phase $\Phi^{N L}(t)$ (dark blue line) proportional to its intensity envelope. $\Phi^{N L}(t)$ changes the instantaneous frequency of the carrier wave (light blue line).

Fig.(2.1) depicts the nonlinear phase $\Phi^{N L}(t)$ (dark blue line, Eqn.2.23) and corresponding shift of the instantaneous frequency $\delta \omega$ (light blue line, Eqn.(2.24) a Gaussian pulse induces in a medium with $n_{2}>0$. As $\Phi^{N L}(t)$ is proportional to the intensity envelope, $\delta \omega$ is negative in the first half of the pulse and positive at its end. If the magnitude of the spectral modifications becomes comparable to the bandwidth of the incident field, the fundamental spectrum can be significantly broadened or narrowed depending on the initial chirp of the driving wave [34]. As the spectral width of the pulse $\Delta \omega$ is related to its duration $\tau_{p}$ by $\Delta \omega \propto \frac{2 \pi}{\tau_{p}}$, self-phase 
modulation considerably alters the frequency structure of the fundamental if $\Phi_{\max }^{N L} \geq 2 \pi$.

$$
\delta \omega_{\max } \approx \frac{\Phi_{\max }^{N L}}{\tau_{p}} \approx \frac{2 \pi}{\tau_{p}} \rightarrow \Phi_{\max }^{N L} \geq 2 \pi
$$

For a Gaussian pulse $\delta \omega(t)$ reaches its maximum and minimum amplitude at the inflection points of $\Phi^{N L}(t)$ (Fig. (2.1)). In between it can assume the same value at two different instants in time, resulting in an interference modulation of the nonlinearly modified spectrum [34].

\subsection{Self-Focusing}

The intensity dependence of the refractive index (Eqn.(2.22) ) does not only influence the spectral composition of the pulse but also its spatial structure. In the transverse profile of a Gaussian beam the intensity decreases with the distance from the optical axis. This results in a spatially inhomogeneous nonlinear modification of the optical material density across the beam area: for a medium with $n_{2}>0$ the refractive index becomes highest at the beam center and decreases towards its sides. Hence the nonlinear Kerr effect introduces a focusing lens (Fig. (2.2)) in the material. The location of the focus can be calculated with Fermat's principle [33], if the beam diameter is considerably larger than the wavelength of the electric field and diffraction can be neglected. Then the optical path of all rays at different transverse positions in the material must be the same.

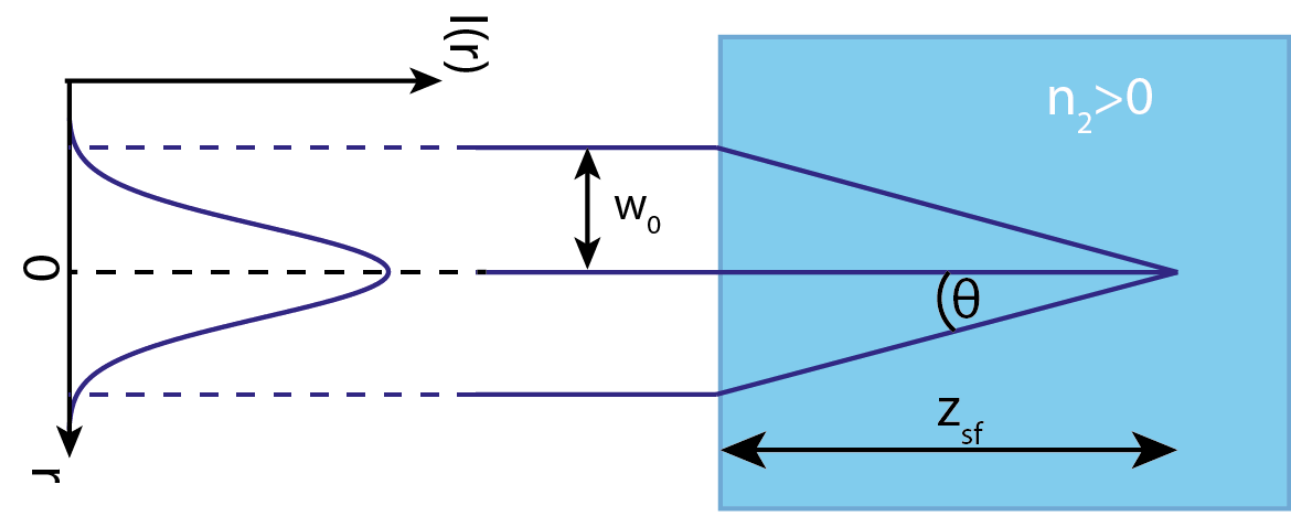

Figure 2.2: The intensity dependent refractive index alters the optical path length for rays at different radial positions. In materials with $n_{2}>0$ the Gaussian intensity distribution leads to self-focusing of the beam after a propagation distance of $z_{s f}$.

Fig. (2.2) illustrates how Fermat's principle determines the focusing angle $\theta$ in the medium. After entering the material the beams at different radial positions meet at the self-focusing 
length $z_{s f}$.

$$
\begin{gathered}
\left(n_{0}+n_{2} I\right) z_{s f}=\frac{n_{0} z_{s f}}{\cos \theta} \\
\cos \theta \approx 1-\frac{1}{2} \theta^{2} \rightarrow \theta=\sqrt{\frac{2 n_{2} I}{n_{0}+n_{2} I}}
\end{gathered}
$$

As $\theta$ is related to the beam radius $w_{0}$ by $\theta=\frac{w_{0}}{z_{s f}}, z_{s f}$ is given by

$$
z_{s f}=w_{0} \sqrt{\frac{n_{0}+n_{2} I}{2 n_{2} I}} .
$$

\subsection{Self-Steepening}

To further investigate the impact of the Kerr effect on the nonlinear light-matter interaction, the factor $\frac{i}{\omega_{0}} \frac{\partial}{\partial \tau}$ on the left hand side of Eqn. 2.17) is now taken into account. The terms $\frac{i k_{1}}{k_{0}} \frac{\partial}{\partial \tau}$ on the right side are further neglected. According to the slowly-evolving-amplitudeapproximation $\left[1+\frac{i}{\omega_{0}} \frac{\partial}{\partial \tau}\right]^{2} \approx 1+\frac{2 i}{\omega_{0}} \frac{\partial}{\partial \tau}$.

The frequency dependence of $\chi^{(3)}$ is taken into account with the lowest order terms of a Taylor expansion

$$
\chi^{(3)}(\omega) \approx \chi^{(3)}\left(\omega_{0}\right)+\left(\omega-\omega_{0}\right) \frac{d \chi^{(3)}}{d \omega}[36] .
$$

Back in the laboratory reference frame Eqn.2.17) can be written as

$$
\begin{gathered}
\frac{\partial A(\vec{r}, t)}{\partial z}-k_{1} \frac{\partial A(\vec{r}, t)}{\partial t}=\frac{i}{2 k_{0}} \Delta_{\perp} A(\vec{r}, t)-\frac{i k_{2}}{2} \frac{\partial^{2} A(\vec{r}, t)}{\partial t^{2}}+\frac{3 i \omega_{0}}{2 n_{0} c} \chi^{(3)}\left(\omega_{0}\right)|A(\vec{r}, t)|^{2} A(\vec{r}, t)+ \\
\frac{3 i \omega_{0}}{2 n_{0} c} \chi^{(3)}\left(\omega_{0}\right)\left[2+\frac{\omega_{0}}{\chi^{(3)}\left(\omega_{0}\right)} \frac{d \chi^{(3)}}{d \omega}\right] \frac{i}{\omega_{0}} \frac{\partial}{\partial t}|A(\vec{r}, t)|^{2} A(\vec{r}, t) .
\end{gathered}
$$

The first three terms on the right hand side have been previously identified as the contribution of diffraction, group velocity dispersion and self-phase modulation, respectively. By 
computing $\frac{\partial}{\partial t}\left(|A(\vec{r}, t)|^{2} A(\vec{r}, t)\right)=2|A(\vec{r}, t)|^{2} \frac{\partial A(\vec{r}, t)}{\partial t}+A(\vec{r}, t)^{2} \frac{\partial A \cdot(\vec{r}, t)}{\partial t}$ Eqn. (2.29) becomes

$$
\begin{aligned}
\frac{\partial A(\vec{r}, t)}{\partial z}-\overbrace{\left[k_{1}+\frac{4 \gamma c}{\omega_{0}}|A(\vec{r}, t)|^{2}\right]}^{n_{\text {eff }}^{(g)} / c} \frac{\partial A(\vec{r}, t)}{\partial t}=\frac{i}{2 k_{0}} \Delta_{\perp} A(\vec{r}, t)-\frac{i k_{2}}{2} \frac{\partial^{2} A(\vec{r}, t)}{\partial t^{2}}+ \\
+\quad \frac{3 i \omega_{0}}{2 n_{0} c} \chi^{(3)}\left(\omega_{0}\right)|A(\vec{r}, t)|^{2} A(\vec{r}, t)-\frac{2 \gamma}{\omega_{0}} A(\vec{r}, t)^{2} \frac{\partial A(\vec{r}, t)}{\partial t}
\end{aligned}
$$

with $\gamma=\frac{3 \omega_{0}}{2 n_{0} c} \chi^{(3)}\left(\omega_{0}\right)\left[1+\frac{\omega_{0}}{2 \chi^{(3)}\left(\omega_{0}\right)} \frac{d \chi^{(3)}}{d \omega}\right]$. The second term on the left hand side shows that an intensity dependent contribution to the inverse group velocity emerges in the nonlinear light-matter interaction. It can be described with the nonlinear group index $n_{2}^{(g)}$ which is related to the effective group velocity index by $n_{\text {eff }}^{(g)}=n_{0}^{(g)}+n_{2}^{(g)} I$.

$$
n_{2}^{(g)}=\frac{12}{n_{0}^{2} c \epsilon_{0}} \chi^{(3)}\left(\omega_{0}\right)\left[1+\frac{\omega_{0}}{2 \chi^{(3)}\left(\omega_{0}\right)} \frac{d \chi^{(3)}}{d \omega}\right]
$$

For $n_{2}^{(g)}>0, v_{g}$ decreases proportional to the intensity envelope of the driving field. Hence the central part of the pulse is decelerated to a higher extent than its flanks. This delays the first moment of the pulse and increases the slope at the trailing flank. The pulse front is flattened. The described phenomena is known as self-steepening and illustrated in Fig. (2.3 B) [33]. For few-cycle pulses (in dispersionless media) self-steepening can increase the slope at the pulse end to infinity and form an optical shock wave (Fig.(2.3 C)). When the central part of the pulse is even further delayed and overtaken by the components at the tail, the pulse breaks similar to the behavior known from water waves [37].

The analysis of the nonlinear interaction presented so far illustrates that various physical mechanisms influence the propagation of an intense light pulse through matter (Eqn. (2.30)). The characteristic length scales on which the different effects significantly modify the pulse properties can be used to classify the relevance of the contribution. If the characteristic length of the nonlinear effect is small, its impact in the interaction is high.

The dispersion length $L_{\text {dispersion }}$ constitutes a measure for the amplitude of group velocity dispersion [33]

$$
L_{\text {dispersion }}=\frac{\tau_{p}^{2}}{4 \ln (2)\left|k_{2}\right|} .
$$

The impact of self-phase modulation can be estimated with the nonlinear length $L_{N L}$ [34]

$$
L_{N L}=\frac{c}{\omega n_{2} I}
$$




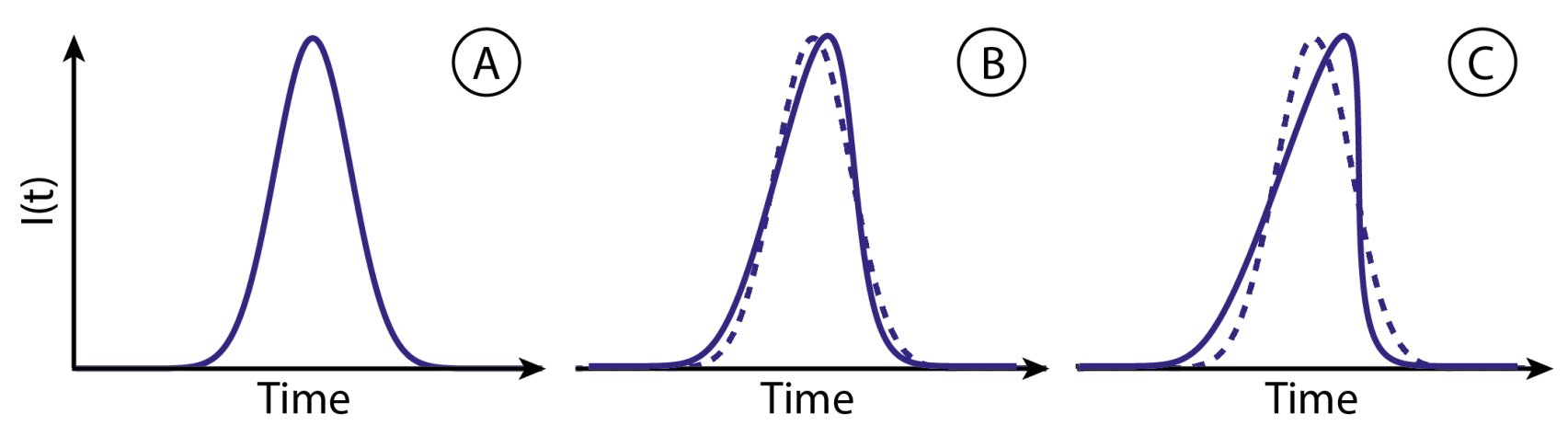

Figure 2.3: Panel A: The intensity envelope of a Gaussian pulse. Panel B: The intensity envelope of the Gaussian pulse is altered by self-steepening in a material with $n_{2}^{(g)}>0$. Panel C: For ultrashort pulses severe self-steepening can induce the formation of optical shock waves.

For self-steepening the self-steepening distance $L_{S S}$ is defined

$$
L_{S S}=\frac{c \tau_{p}}{2 \sqrt{\ln (2)} n_{2}^{(g)} I} .
$$

\subsection{Frequency Tripling}

For reasons of lucidity only components of the nonlinear polarization at the fundamental frequency $\omega$ of the driving field have been considered so far (Eqn.(2.18)). However in the nonlinear light-matter interaction also higher order harmonics $n \cdot \omega(n \in N)$ are generated (Eqn.(2.4)). In the $\chi^{(3)}$ process, 3 photons of the fundamental can be transformed into coherent radiation oscillating at a frequency of $3 \omega$ (Fig.(2.4) [33].

$$
P^{(3)}(t)=\epsilon_{0} \chi^{(3)} E(t)^{3}=\epsilon_{0} \chi^{(3)} A(t)^{3} \exp (-i(3 \omega t))
$$

To evaluate the efficiency of the nonlinear process the magnitude of $\left|\overleftrightarrow{\chi}^{(i)}\right|$ (Eqn.(2.35) is estimated. The interatomic atomic force $E_{a t}$ can be approximated with

$$
E_{a t}=\frac{e}{4 \pi \epsilon_{0} a_{0}^{2}}=5.14 \cdot 10^{11} \frac{\mathrm{V}}{\mathrm{m}}
$$

where $e=1.6 \cdot 10^{-19} \mathrm{C}$ is the electron charge, $\epsilon_{0}=8.85 \cdot 10^{-12} \frac{F}{m}$ the electric permittivity of free space and $a_{0}=5.29 \cdot 10^{-11} \mathrm{~m}$ the Bohr radius. If the amplitude of the external driving field is of the same order of magnitude as $E_{a t}$, the amplitude of the linear material response 


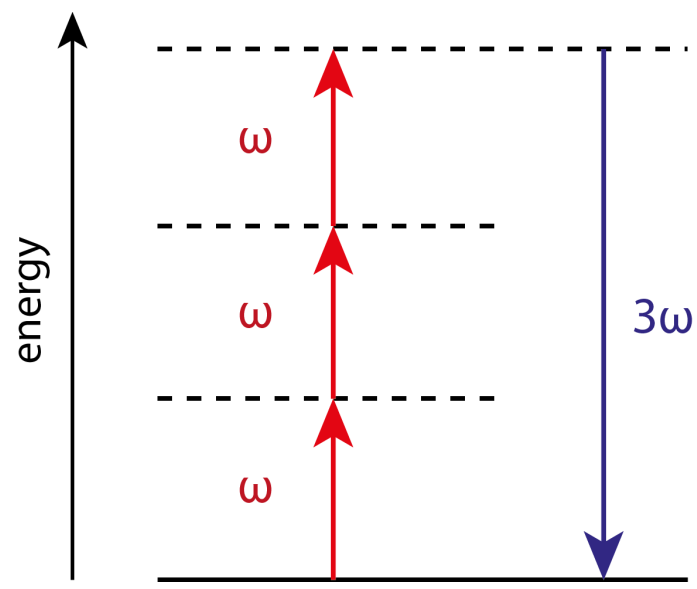

Figure 2.4: Schematic principle of frequency tripling [33]: 3 photons of the fundamental wave with frequency $\omega$ are absorbed by a two level system. In return a high energy photon with a frequency of $3 \omega$ is emitted.

$P^{(L)}(t)$ (Eqn.2.3) is of similar size as for $P^{(N L)}(t)$ (Eqn. 2.4 ). As $\chi^{(1)} \approx 1$

$$
\epsilon_{0} \cdot \chi^{(1)} \cdot E_{a t} \approx \epsilon_{0} \cdot \chi^{(i)} \cdot E_{a t}^{i} \rightarrow \chi^{(i)}=\frac{1}{E_{a t}{ }^{i-1}} .
$$

With increasing order of the nonlinearity $\chi^{(i)}$ decreases [38]. For second order processes $\chi^{(2)} \approx 19.5 \cdot 10^{-13} \frac{\mathrm{m}}{\mathrm{V}}$. For third order nonlinearities the efficiency drops considerably as

$\chi^{(3)} \approx 38.0 \cdot 10^{-24} \frac{\mathrm{m}^{2}}{V^{2}}$. The conversion rate for frequency tripling of VIS/NIR photons into the UV range in the gaseous phase is less than $<1 \%$.

\subsection{Intrapulse Raman-Scattering}

As electrons possess less mass than the ionic cores most electronic excitations in solids oscillate at higher frequencies than the vibrations of the lattice. This is the reason why the interaction of ultrashort pulses with matter can be assumed to be dominated by the electronic contribution. However the extent to which Raman modes contribute to the induced polarization is still unclear [34].

The principle of spontaneous inelastic Raman scattering is displayed in Fig.(2.5). An external pump field at frequency $\omega$ excites electrons from the ground state into a virtual level associated with an excited state. The electron decays into a vibrational level by emitting a photon at the Stokes-frequency $\omega_{\text {Stokes }}<\omega$. As the final vibration state is located energetically above the ground state energy is transferred from the external field to the material (Fig.(2.5A)). In the anti-Stokes process the electron is excited from the vibrational state into the virtual level by the external field and decays into the ground state. Thereby it emits an Anti-Stokes photon $\omega_{\text {Anti-Stokes }}>\omega$. In this case the pump field gains energy from the medium (Fig. (2.5B)). 

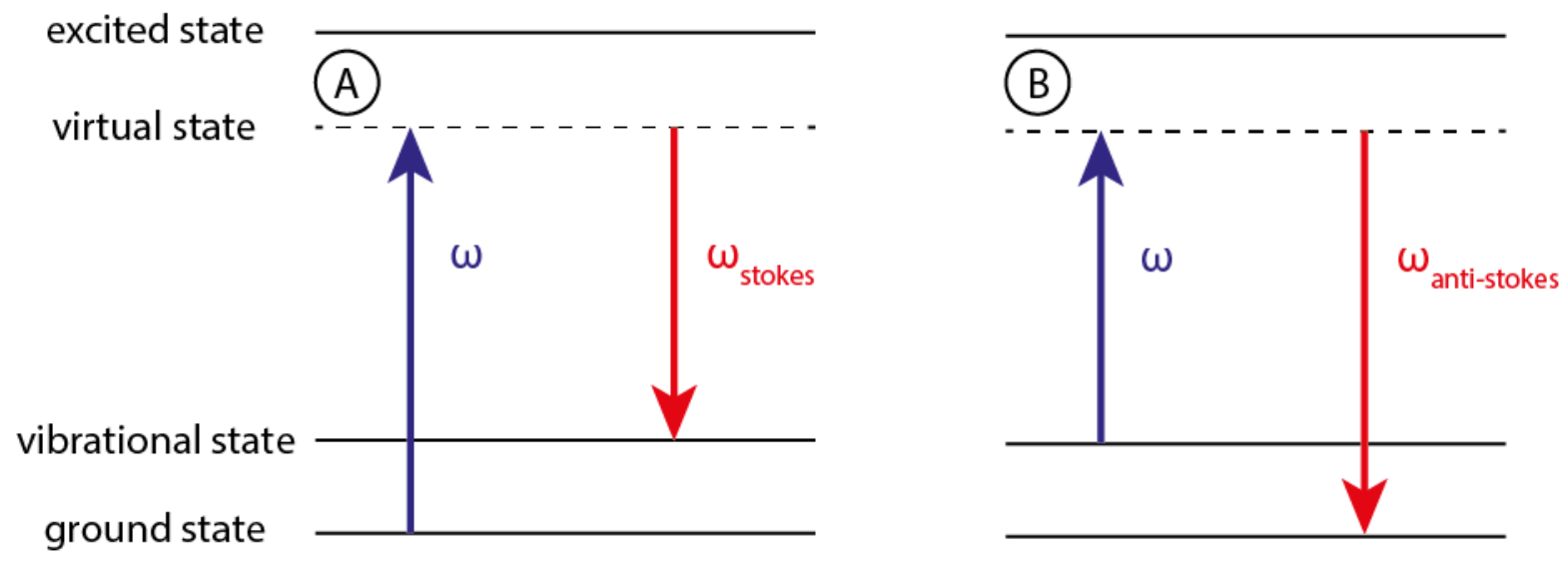

Figure 2.5: The principle of spontaneous Raman scattering for the Stokes (Panel A) and Anti-Stokes (Panel B) process.

However as the electron decays into the populated ground state, the transition probability for Anti-Stokes scattering is by orders of magnitude smaller than for the Stoke process. The cross section of both Raman processes can be considerably increased by exposing the energy system to radiation with $\omega_{\text {Stokes }}$ or $\omega_{\text {Anti-Stokes }}$ additionally to the pump field $\omega$ [34].

An ultrashort few-cycle optical pulse covers a bandwidth of $>1$ octave. Therefore it can deliver $\omega$ and $\omega_{\text {Stokes }}$ for stimulated Stokes scattering. In the Raman process energy is transferred from the high frequency part of the pulse spectrum to the long wavelength range. If the lifetime of the virtual level lies in the femtosecond range, intensity is redistributed from the center of the pulse to its red shifted tail. This phenomena is known as intrapulse Raman-scattering [34].

Absorption can be included in Eqn.(2.5) under consideration of the general relation between the permittivity $\epsilon$ and $n$ with the absorption coefficient $\alpha$

$$
\epsilon=\left(n+\frac{i \alpha c}{2 \omega}\right)^{2}
$$

The time delayed Raman decay and its impact on the pulse structure can be considered by the temporal and dispersive profile of $\chi^{(3)}[34$.

\subsection{Plasma Dynamics}

\subsubsection{The Contribution of Free Electrons to the Refractive Index}

As presented in the previous sections many nonlinear optical phenomena resulting from the oscillatory motion of electrons around their equilibrium position induced by a strong external field are well described with the pertubative approach (Eqn.(2.4)). If the amplitude of the incident field becomes comparable to the interatomic forces $E_{a t}$ (Eqn.(9.7)) this model breaks 
down as the electronic structure of the medium is altered considerably when the carriers gain sufficient energy to escape the attractive potential of their parent ion. To understand the dynamics in a plasma it is instructive to study the classical motion of a free electron with mass $m_{e}$ and charge $e$ under the influence of an external electric field $E(t)=E(t) \widehat{x}$. It is well described by the harmonic oscillator model [33].

$$
\begin{gathered}
m_{e} \ddot{x}=-e E(t) \\
E(t)=E_{0} \exp (-i \omega t)+c c .
\end{gathered}
$$

$x(t)$ is the position of the electron in direction of the oscillating wave $E(t)$. This differential equation is fulfilled if the electron follows $E(t)$ instantaneously on the trajectory $x(t)=$ $x_{0} \exp (-i \omega t)+c c$. with a maximum amplitude of $x_{0}=\frac{e E_{0}}{m_{e} \omega^{2}}$.

On the one hand the polarization is the average over all induced dipole momenta $d(x)=$ $-e x(t)$. For one electron $P(t)=-e x(t)$. On the other hand according to Eqn.(2.3) $P$ is given by $P(t)=\epsilon_{0} \alpha(\omega) E(t)$ with the molecular polarizability $\alpha(\omega) .(\alpha(\omega)$ is the polarizability of a single dipole, for $\mathrm{N}$ charges $\chi^{(1)}=N \alpha(\omega)$ ) [33]. By exploiting this identity $\alpha(\omega)$ can be written as

$$
\alpha(\omega)=-\frac{e^{2}}{m_{e} \epsilon_{0} \omega^{2}}
$$

The permittivity $\epsilon$ of an ensemble of free electrons with the carrier density $n_{e}$ is connected to the polarizability by

$$
\epsilon(\omega)=1+n_{e} \alpha(\omega)=1-\frac{n_{e} e^{2}}{m_{e} \epsilon_{0} \omega^{2}}=1-\frac{\omega_{p}^{2}}{\omega^{2}}
$$

with the plasma frequency $\omega_{p}^{2}=\frac{n_{e} e^{2}}{m_{e} \epsilon_{0}}$.

In the absence of absorption the refractive index $n$ is connected to $\epsilon=n^{2}$. For small changes $\Delta n<<1$ and materials with $n \approx 1$

$$
\begin{gathered}
n^{2}=n^{2}+\Delta n^{2}+2 n \Delta n \approx 1+2 n \Delta n \\
1+2 n \Delta n=1-\frac{n_{e} e^{2}}{m_{e} \epsilon_{0} \omega^{2}} \rightarrow \Delta n=-\frac{n_{e} e^{2}}{2 n m_{e} \epsilon_{0} \omega^{2}}<0
\end{gathered}
$$

the free electron contribution decreases the refractive index of the material [33]. 


\subsubsection{The Nonresonant Contribution of Bound Electrons to the Refractive Index}

The model of the harmonic oscillator can also be employed to derive the modification of the refractive index by bound electrons. In this case a term representing the restoring force of the atomic potential is introduced in Eqn. (2.39). $\omega_{0}$ is the resonance frequency of the system.

$$
\begin{gathered}
m_{e} \ddot{x}+m_{e} \omega_{0}^{2} x=-e E(t) \\
E(t)=E_{0} \exp (-i \omega t)+c c
\end{gathered}
$$

Eqn. 2.45 is solved by $x(t)=\frac{e E(t)}{m_{e}\left(\omega^{2}-\omega_{0}^{2}\right)}$. Analogue to the derivation presented in the previous section, far off resonance $\omega<<\omega_{0}$ the polarizability of the system is found to be

$$
\alpha(\omega)=\frac{e^{2}}{m_{e} \epsilon_{0} \omega_{0}^{2}} .
$$

According to Eqn.(2.42) and Eqn.(2.41) the bound electrons alter the refractive index by

$$
\Delta n=\frac{n_{e} e^{2}}{2 n m_{e} \epsilon_{0} \omega_{0}^{2}}>0 .
$$

It is apparent that the effect of bound and free carriers on the optical material density is of opposite sign. The plasma contribution (Eqn.(2.44) decreases the refractive index, whereas the response of bound carriers (Eqn.(2.48) ) increases its value. The absolute value of the free electron contribution exceeds the magnitude of the bound carrier effect. This difference is intuitively comprehensible as the excursion of the induced free carrier motion is bigger than the path length covered by the oscillating bound charges [33].

\subsubsection{The Polarization in a Plasma}

If collective particle motion, collision processes and relativistic effects are neglected the nonlinear polarization in an underdense plasma $\left(\omega_{p}<\omega\right)$ is the sum over all the induced dipole momenta. However the contribution of the ionic cores can be neglected as they are by orders of magnitude heavier and therefore slower than the freed electrons [39]. This means that the nonlinear polarization can be written as

$$
P_{N L}(t)=n_{e}(t) e\langle x(t)\rangle
$$

where $\langle x(t)\rangle$ is the expectation value of the electronic wavefunction. The temporal derivative of $P_{N L}(t)$ is given by

$$
\frac{d P_{N L}(t)}{d t}=\frac{d n_{e}(t)}{d t} e x_{0}(t)+n_{e}(t) e \frac{d\langle x(t)\rangle}{d t} .
$$


$x_{0}(t)=\frac{I_{p}}{e E(t)}$ is the distance from the ionic core at which the electron appears after tunneling through the atomic potential suppressed by the external electric field [39]. The two terms on the right hand side of Eqn.(2.50) represent the different physical processes contributing to the nonlinear polarization response. The first term describes the ionization rate at which new electrons appear outside the Coulomb barrier at position $x_{0}$. Ionization happens with a maximum probability at the peaks of the driving field in each subcycle. The second term accounts for the acceleration of the freed carriers in the field. They acquire their maximum velocity at the zero crossings of the oscillating wave [39]. 


\section{Chapter 3}

\section{Time Integrated Investigation of the Nonlinear Kerr Coefficient}

Most of the nonlinear phenomena described in section 2 are well understood if the oscillation period of the exciting field clearly exceeds the time constant of the induced dynamics [40]. As laser pulses with femtosecond duration are in most cases shorter than the oscillation period of acoustic and typical optical phonons in solids [34], the established values of the nonlinear Kerr coefficient $n_{2}$ (section 2.1) loose validity [3], [41]. To estimate the magnitude of the optical Kerr effect in the interaction of the few cycle pulses used in this study with dielectrics, $n_{2}$ is determined with two different time integrating techniques. On the one hand the well-established Z-scan method is employed [42]. On the other hand a concept based on the detection of the intensity dependent reflectivity is developed. It is particularly suitable for broadband few-cycle laser pulses.

\subsection{Z-Scan}

A well-proven concept for the determination of the nonlinear Kerr coefficient is the Z-scan technique introduced by M. Sheik-Bahae in 1990 [42]. It makes use of the spatial implications of the intensity dependent refractive index described in section 2.2. The basic setup is depicted in Fig.(3.1): The investigated target is positioned in the focused laser beam of few-cycle VIS/NIR pulses (appendix 9.1.1). The Rayleigh length clearly exceeds the sample thickness by several orders of magnitude. The transmitted beam is split into two arms: in the open aperture arm its power is recorded. In the small aperture arm a pinhole is positioned in the far field of the laser beam and the transmitted power detected on a powermeter. When the sample is moved stepwise along the optical axis towards the focus, the intensity it is exposed to grows. The transverse Gaussian intensity profile of the beam inhomogeneously modifies the optical material density and introduces a nonlinear lens (section 2.2). With increasing peak intensity the focal length of this lens decreases and the self focusing of the beam becomes more pronounced. While the target is situated in front of the geometrical focus 
the additional nonlinear lens decreases the effective focal length and rises the divergence of the transmitted beam (Fig.(3.2 A)). Therefore less power is transmitted through the pinhole in the small aperture arm. As soon as the target is located behind the geometrical focus the Kerr lens reduces the divergence of the transmitted radiation, increasing the power measured in the small aperture arm (Fig.(3.2 B)). In the absence of nonlinear absorption the power in the open aperture arm remains unaffected by the light-matter interaction.

The Z-scan is performed on $\ell=50 \mu \mathrm{m}$ thick amorphous $\mathrm{SiO}_{2}$ and crystalline $\mathrm{Al}_{2} \mathrm{O}_{3}$ samples. To determine the intensity the sample is exposed to the power and beam size are recorded. Additionally the pulse duration is extracted from an independent measurement (appendix 9.5). The peak intensity in the geometrical focus is set to $I=(1.7 \pm 0.3) \cdot 10^{13} \frac{\mathrm{W}}{\mathrm{cm}^{2}}$. In order to avoid spatial and temporal distortion of the pulse by interaction with air the $\mathrm{Z}$-scan is performed in vacuum $\left(10^{-4} \mathrm{mbar}\right)$.

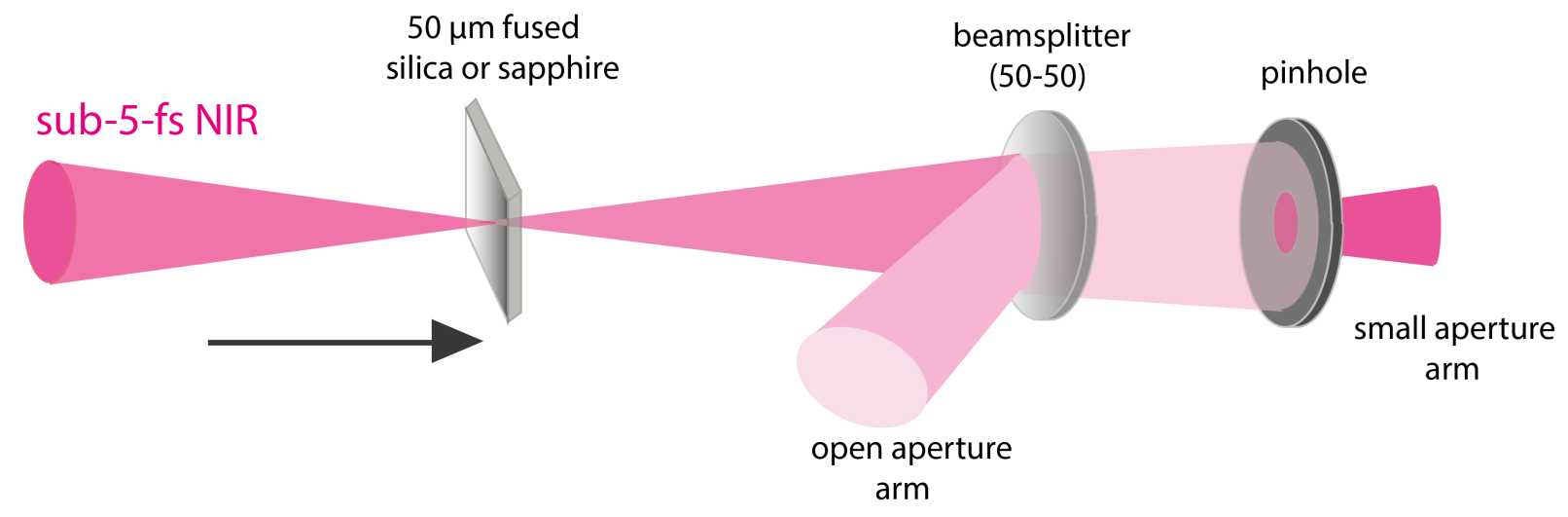

Figure 3.1: Schematic setup of the Z-scan to determine the nonlinear Kerr coefficient $n_{2}$.

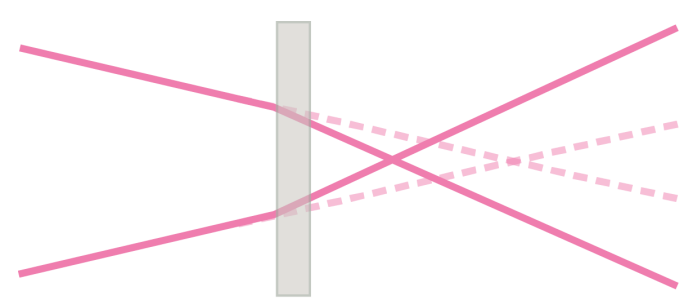

(A) sample positioned in front of geometrical focus

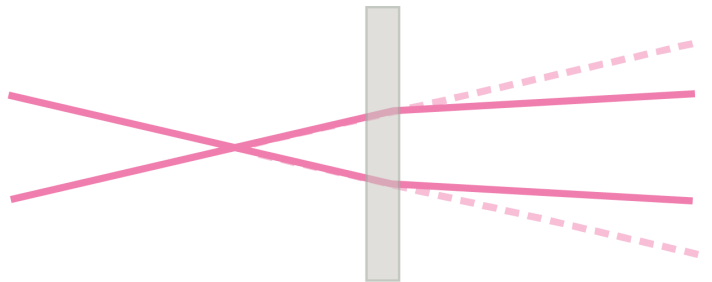

(B) sample positioned behind geometrical focus

Figure 3.2: The impact of self focusing on the effective focal length. Panel A: When the target is positioned in front of the geometrical focus the additional Kerr lens decreases the effective focal length and the divergence of the transmitted beam grows. Panel B: When the sample is located behind the geometrical focus self focusing decreases the beam divergence.

The traces recorded with $\ell=50 \mu \mathrm{m}$ thick fused silica plates are depicted in Fig.(3.3). Panel A displays the power measured through the pinhole in the small aperture arm. It 
decreases with growing strength of the Kerr lens while the sample is moved towards the geometrical focus. After passing through the focus the transmission increases and finally returns to its original value. Panel B shows the power recorded in the open aperture arm, which is constant throughout the entire interaction. This verifies the absence of multi photon absorption and a negligible contribution of frequency conversion to the induced nonlinear process (section 2.4] [42]. If spectral changes of the pump field become non negligible, it is no longer sufficient to exclusively examine the modification of the spatial beam properties to access the Kerr dynamics. To compensate for intensity fluctuations of the pump field the small aperture signal is normalized with the open aperture trace. The result is displayed in Fig.(3.3 C, blue line).
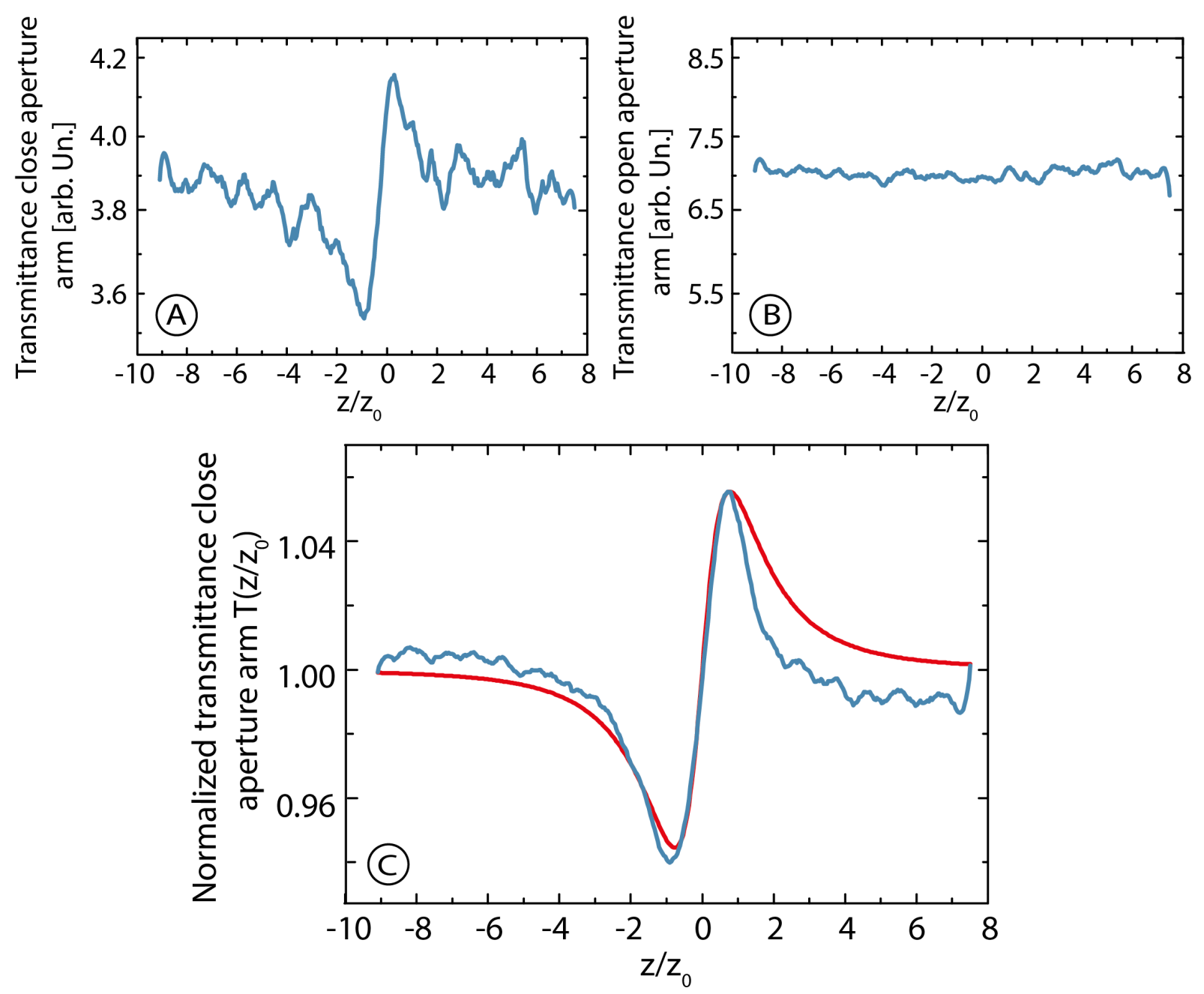

Figure 3.3: The power recorded in the open (Panel A) and small aperture arm (Panel B) in the Z-scan measurement with $\ell=50 \mu \mathrm{m}$ thick fused silica plates. The normalized open aperture signal (Panel C, blue line) is fitted (red line) with Eqn.(3.1) to extract the nonlinear Kerr coefficient [42].

The normalized small aperture signal $T\left(\frac{z}{z_{0}}\right)$ can be employed to extract the nonlinear Kerr 
coefficient with the formalism proposed by M. Sheik-Bahae [42], [43].

$$
\begin{gathered}
T\left(\frac{z}{z_{0}}\right)=1-\frac{4 \Delta \Phi \frac{z}{z_{0}}}{\left(\left(\frac{z}{z_{0}}\right)^{2}+9\right)\left(\left(\frac{z}{z_{0}}\right)^{2}+1\right)} \\
\Delta \Phi=k \ln _{2} I
\end{gathered}
$$

The Rayleigh length $z_{0}$, the peak intensity $I$ and the sample thickness $\ell$ are determined experimentally.

The calculated fit is depicted as the red line in Fig.(3.3 C) and yields a result of $n_{2}=$ $(1.1 \pm 0.3) \cdot 10^{-16} \frac{\mathrm{cm}^{2}}{W}$. The deviation between the experiment data and the fit behind the geometrical focus $\left(\frac{z}{z_{0}}=2-8\right)$ is caused by small distortions of the wavefront of the pump field at these positions. The obtained result for the nonlinear Kerr coefficient is a factor of $2-3$ smaller than the $n_{2}$ values reported in literature for the interaction of fused silica with multi-cycle fields [3], [41].

The value of the nonlinear Kerr coefficient strongly depends on the spectral and temporal profile of the exciting laser field. Different physical mechanisms contribute to the nonlinear interaction and are summarized in the empirical variable $n_{2}$. The individual contributions evolve on different timescales: as electrons are the lighter than the ionic cores they oscillate with higher frequencies than lattice vibrations. Only if the timing constant of the nonlinearity is comparable to the interaction period the induced dynamics influence the light field. With decreasing pulse duration the interaction time with the material is reduced. Low frequency polarization components do no longer contribute to the Kerr coefficient. As laser pulses with sub-picosecond duration are shorter than the oscillation period of typical acoustic phonons, the $n_{2}$ measured in the interaction with these fields are smaller than values reported for nanosecond pulses [34]. Oguama et al. attempted to disentangle the electronic and Raman contribution to the nonlinear dynamics induced by picosecond pulses in fused silica in 2005. According to their findings the contribution of optical phonons increases the value of $n_{2}$ by $18-20 \%$ [44]. The results reported here for sub- 4 ss laser pulses suggest that the nonlinear polarization is dominated by the electronic response of the material .

The Z-scan is repeated under identical conditions for $\ell=50 \mu \mathrm{m}$ thick $\mathrm{Al}_{2} \mathrm{O}_{3}$ plates. The crystalline samples are cut along the $C$ plane perpendicular to the optical axis to avoid birefringence. The normalized signal of the small aperture arm (blue line) and the curve fitted with Eqn.(3.1) (red line) are depicted in Fig.(3.4). For the nonlinear Kerr coefficient a value of $n_{2}=(1.3 \pm 0.3) \cdot 10^{-16} \frac{\mathrm{cm}^{2}}{\mathrm{~W}}$ is extracted. As in the case of fused silica this result is a factor of $2-3$ smaller than the references reported in literature for longer pulses [45]. Fused silica and sapphire are both wide-gap dielectrics with a band gap of similar size $\left(\Delta E_{g} \mathrm{Al}_{2} \mathrm{O}_{3} \approx 8.8 \mathrm{eV}\right.$, $\left.\Delta E_{\mathrm{g} \mathrm{SiO}_{2}} \approx 9 \mathrm{eV}\right)$. Apart from that they share no common features as their energetic structure and chemical and crystalline composition differ significantly. Even though for both materials the value of the Kerr coefficient in the interaction with ultrashort light fields is considerably decreased compared to the interaction with longer pulses. This result suggests that the phenomena is universal in wide-gap solids. The nonlinear dynamics induced by intense 
optical few-cycle pulses in dielectrics in general seem to be dominated by the electronic material response.

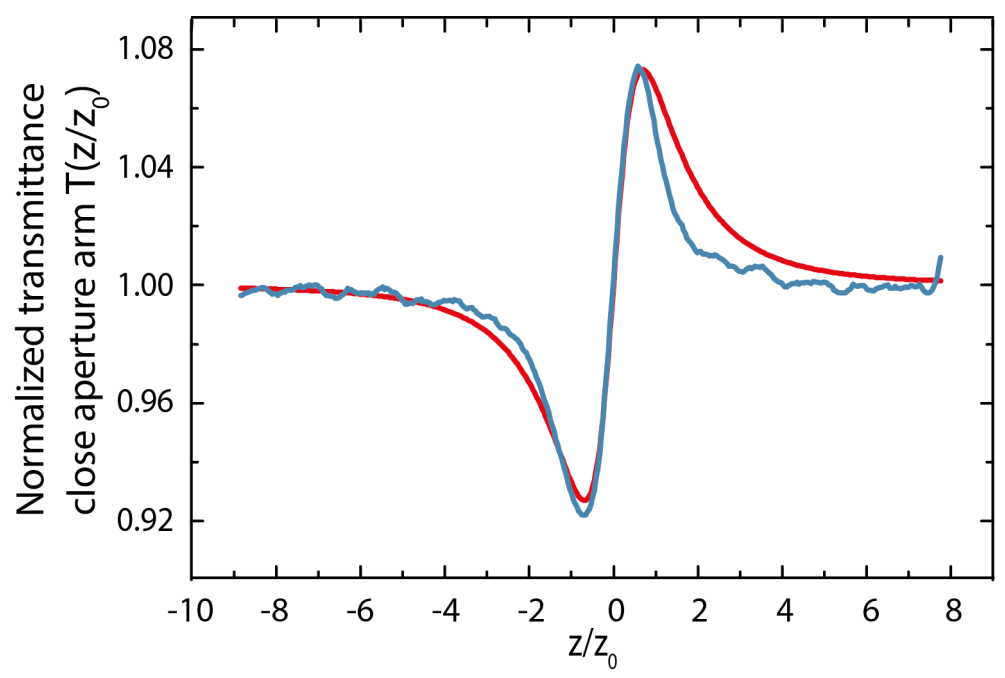

Figure 3.4: The normalized small aperture signal (blue line) recorded in the Z-scan measurement with $\ell=50 \mu m$ thick sapphire plates is fitted (red line) with Eqn.(3.1) to access $n_{2}$ in the interaction with optical few-cycle pulses [42].

\subsection{Intensity Dependent Reflectivity}

The linear propagation of a light pulse through dispersive matter modifies its temporal properties. As ultrashort pulses cover a broad spectral bandwidth their characteristic dispersion length (Eqn.(2.32) ) amounts to a few $100 \mu \mathrm{m}$ in most dielectrics [33]. Hence to reliably determine the nonlinear Kerr coefficient for ultrashort pulses with the Z-scan technique samples with $\mu m$ thickness are required. The fabrication of these thin plates is challenging and expensive. To avoid this inconvenience it is desirable to develop a technique which is based on the detection of the nonlinear modifications of the radiation reflected from the surface instead of the light transmitted through the material. As it is not clear whether the nonlinearity induced at the surface shares identical features with the bulk dynamics this new method has to be validated and verified. Therefore additionally to the Z-scan [42] the intensity dependent reflectivity of the pump field is investigated. The setup is schematically sketched in Fig. (5.1). The dielectric sample is positioned in the focused laser beam of the same optical few-cycle pulses used in the Z-scan (appendix 9.1.1). By translating the material along the optical axis towards the focus, the intensity on the medium is increased. The reflectivity off the front surface is recorded as a function of the intensity the material is exposed to. To spatially separate the reflection from the front and the back surface a wedged sample (with an opening angle of approximately $3^{\circ}$ ) is employed. To optimize the signal-to-noise ratio the 
target is positioned in Brewster's angle.

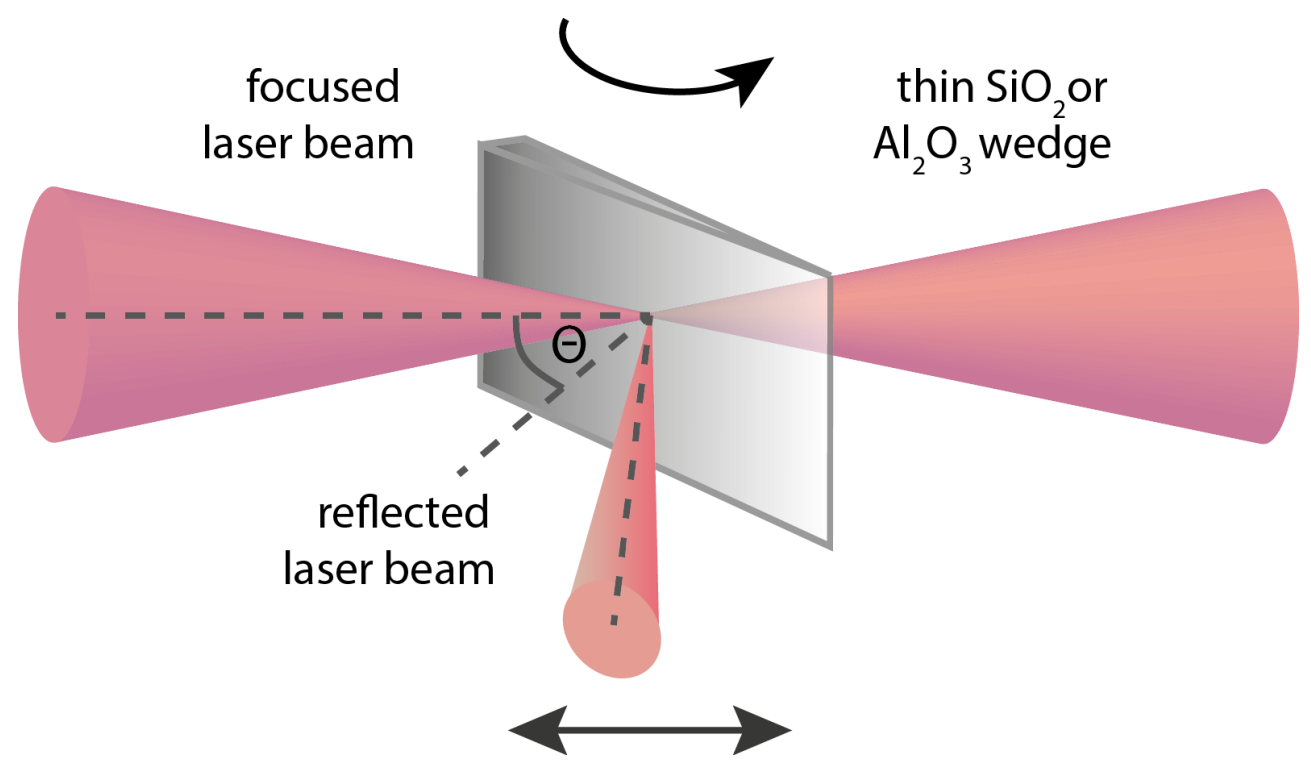

Figure 3.5: Schematic setup for the determination of the nonlinear Kerr coefficient $n_{2}$ by investigation of the intensity dependent reflectivity.

According to Fresnel's formula the reflectivity $R$ of linear polarized light oscillating perpendicular to the incidence plane is given by [38]

$$
\begin{gathered}
R(I, \Theta)=\left|\frac{\sqrt{1-\left(\frac{\sin (\Theta)}{n(I)}\right)^{2}}-n(I) \cos (\Theta)}{\sqrt{1-\left(\frac{\sin (\Theta)}{n(I)}\right)^{2}}+n(I) \cos (\Theta)}\right|^{2} \\
n(I)=n_{0}+n_{2} I
\end{gathered}
$$

with the angle of incidence $\Theta$. The intensity $I$ at each position along the optical axis is determined by tracing of the power, the beam profile and the precise pulse duration in an independent pulse duration measurement (appendix 9.5) [46]. As Eqn.(3.3) relates the intensity dependent refractive index $n(I)$ to the modification of the reflected power $R(I, \Theta)$, $n_{2}$ is accessible.

To exclude spatial and temporal distortion of the pulses by nonlinear interaction with air the experiment is performed in vacuum $\left(10^{-4} \mathrm{mbar}\right)$. In practice it is challenging to precisely determine the absolute value of the angle of incidence. A relative change between two different $\Theta$ can be measured more reliably. Therefore the reflectivity scan is recorded for several $\Theta$ varied around Brewster's angle $\left(\Theta_{B} \approx 56^{\circ}\right)$. The traces measured for fused silica are displayed in Fig.(3.6): For each angle of incidence the intensity dependent reflectivity is recorded (blue line) and individually fitted with Eqn.(3.3) (red line) to extract $n_{2}$. The results 
evaluated for each $\Theta$ coincide to $n_{2}=1.1 \cdot 10^{-16} \frac{\mathrm{cm}^{2}}{W}$ within an error of $\Delta n_{2}=0.4 \cdot 10^{-16 \frac{\mathrm{cm}^{2}}{W}}$. The excellent agreement with the value determined in the Z-scan verifies the applicability of the technique. As the reflected beam only penetrates into the top layers of the material, the nonlinear dynamics induced at the surface seem to strongly resemble the polarization response in the bulk.
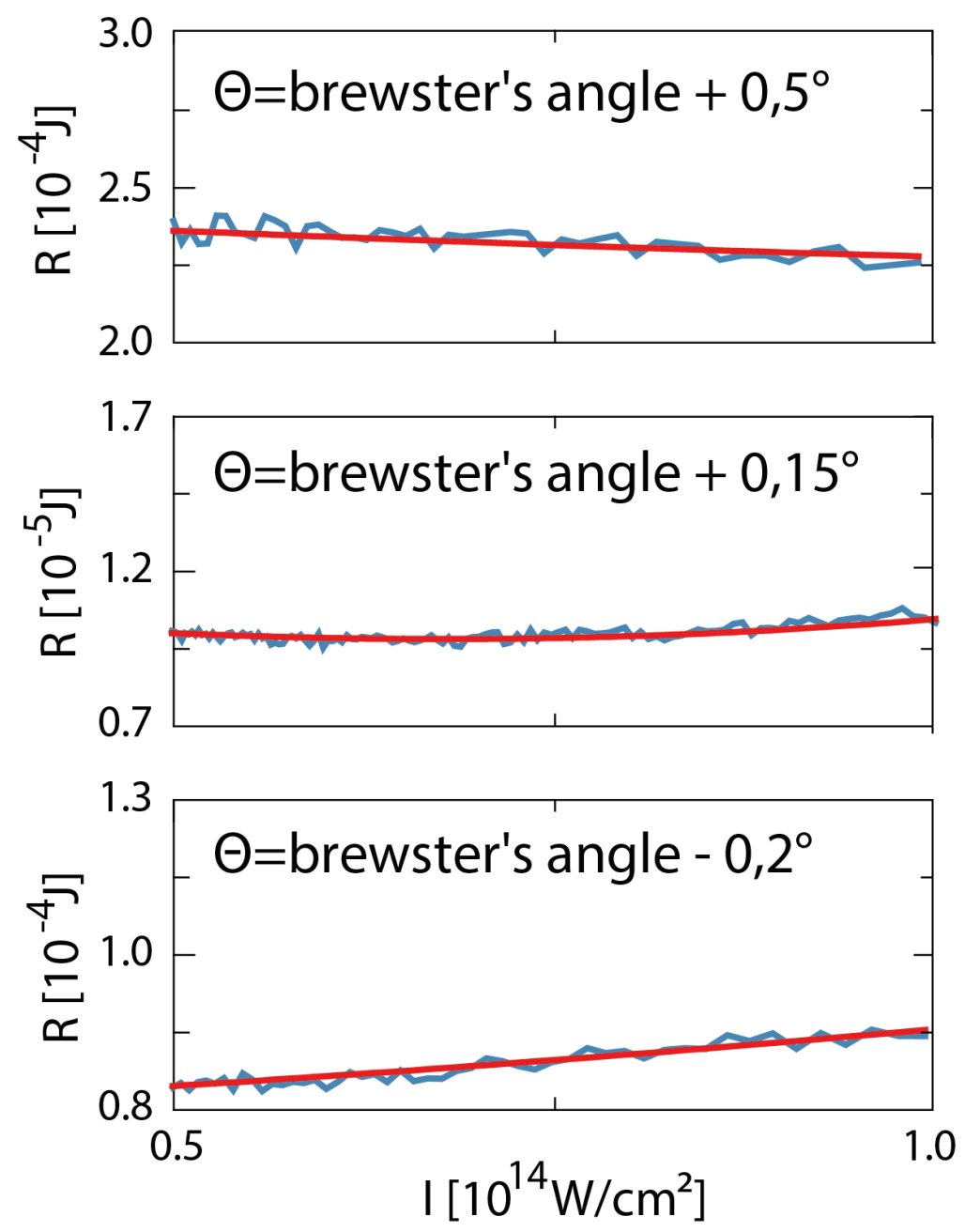

Figure 3.6: The measured intensity dependent reflectivity from a fused silica wedge for different angle of incidence $\Theta$ (blue lines) varied around Brewster's angle are fitted with Eqn.(3.3) (red lines) to extract the nonlinear Kerr coefficient.

The experiment is repeated under identical conditions with a sapphire wedge. Fig.(3.7) depicts the reflectivity of the pump field (blue lines) depending on its intensity on the sample. Again $\Theta$ is varied around Brewster's angle $\left(\Theta_{B} \approx 60^{\circ}\right)$. The curves are fitted with Eqn. (3.3) (red lines) to access the nonlinear Kerr coefficient for each scan. The individual results coincide to $n_{2}=1.3 \cdot 10^{-16 \frac{\mathrm{cm}^{2}}{W}}$ within an error of $\Delta n_{2}=0.4 \cdot 10^{-16 \frac{\mathrm{cm}^{2}}{W}}$. Again the obtained value is identical to the result of the Z-scan.

The results from the Z-scan and the intensity dependent reflectivity measurement hint to 

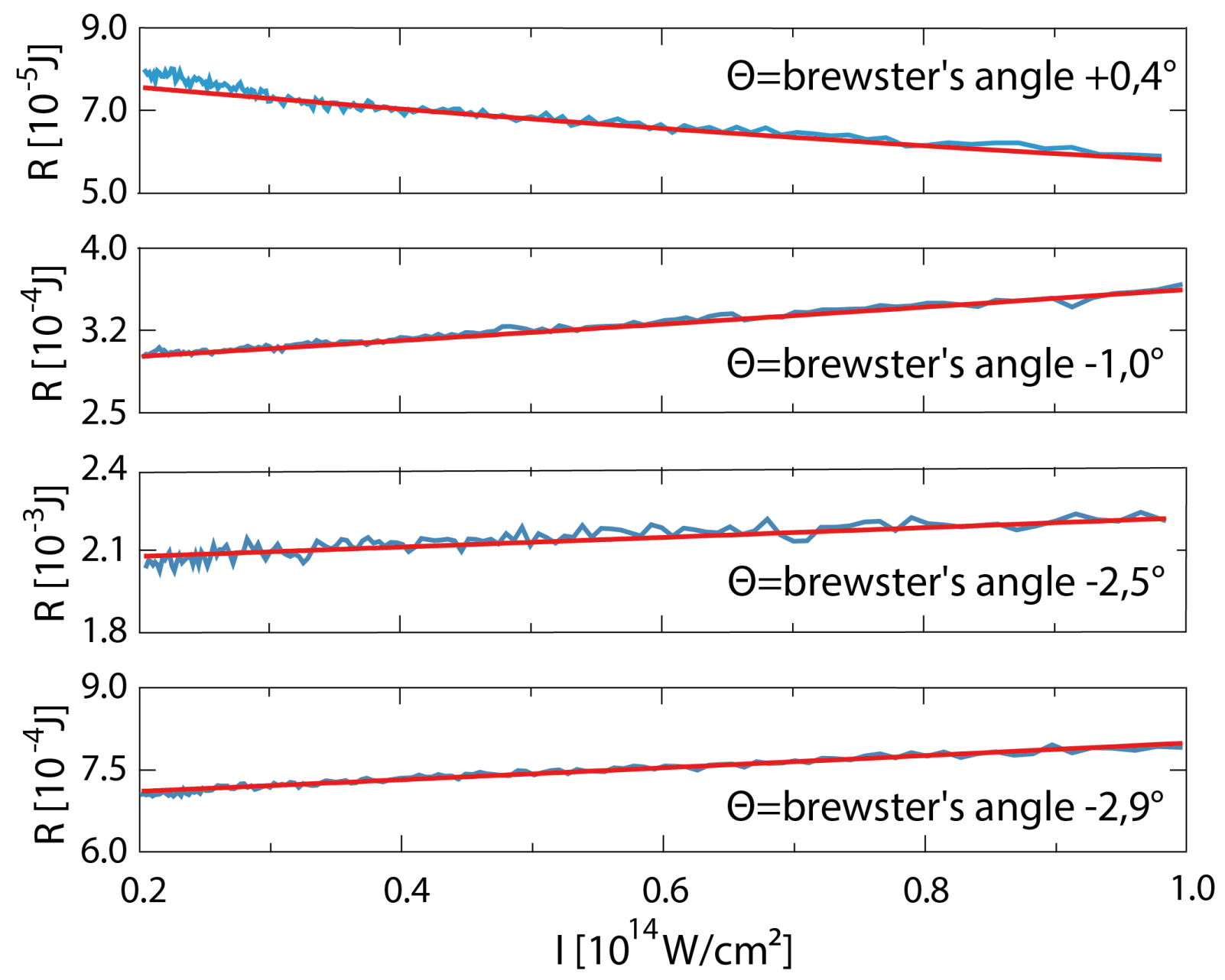

Figure 3.7: The intensity dependent reflectivity from a sapphire wedge is measured at various angle of incidence $\Theta$ (blue lines) which are varied around Brewster's angle. To extract $n_{2}$ the curves are fitted with Eqn.(3.3) (red lines).

the conclusion that the nonlinear response of dielectrics to ultrashort pulses is dominated by the electronic contribution. The dynamics are immediately excited in the first atomic layers at the surface of the material. 


\section{Chapter 4}

\section{Attosecond Transient Absorption Spectroscopy}

As explained in section 1 the aim of this study is to access the nonlinear polarization induced in the strong field interaction in dielectrics by ultrashort pulses. It characterizes the nonlinear response of the medium to the external force and contains information about the underlying physical processes. The time integrated investigation of the Kerr nonlinearity was a first step towards this goal. The observed discrepancy between the nonlinear Kerr coefficient in the interaction with few femtosecond fields and pulses with longer duration indicate that different physical processes contribute to the nonlinearity on different timescales. To explicitly identify them and resolve their dynamics a time resolved experimental technique is required. Electron dynamics in wide-gap materials evolve on sub femtosecond timescales. To track their motion a temporal resolution of a few 100 as is necessary. It is provided by high energy attosecond pulses in the extreme ultraviolet (appendix 9.3). In the following the modification of the spectral absorptivity of these attosecond pulses in the presence of the intense laser field is investigated.

The intense sub-4-femtosecond optical field (appendix 9.1.1) is employed to generate XUV radiation with a pulse duration of less than 100 as (appendix 9.3). The attosecond light burst covers a spectral bandwidth of $>30 \mathrm{eV}$ and is centered at $105 \mathrm{eV}$ (Fig.(4.1) light blue area). The optical pump and XUV probe field propagate collinearly through the sample under scrutiny, a $125 \mathrm{~nm}$ thick fused silica foil. The transmitted XUV light is spectrally resolved and recorded. As displayed in Fig.(4.2 A) the XUV photons excite electrons from the inner core L shell into the broad conduction band of fused silica. Therefore several characteristic absorption lines are visible in the spectrum of the XUV radiation after transmission through the material (Fig.4.1) dark blue line). 


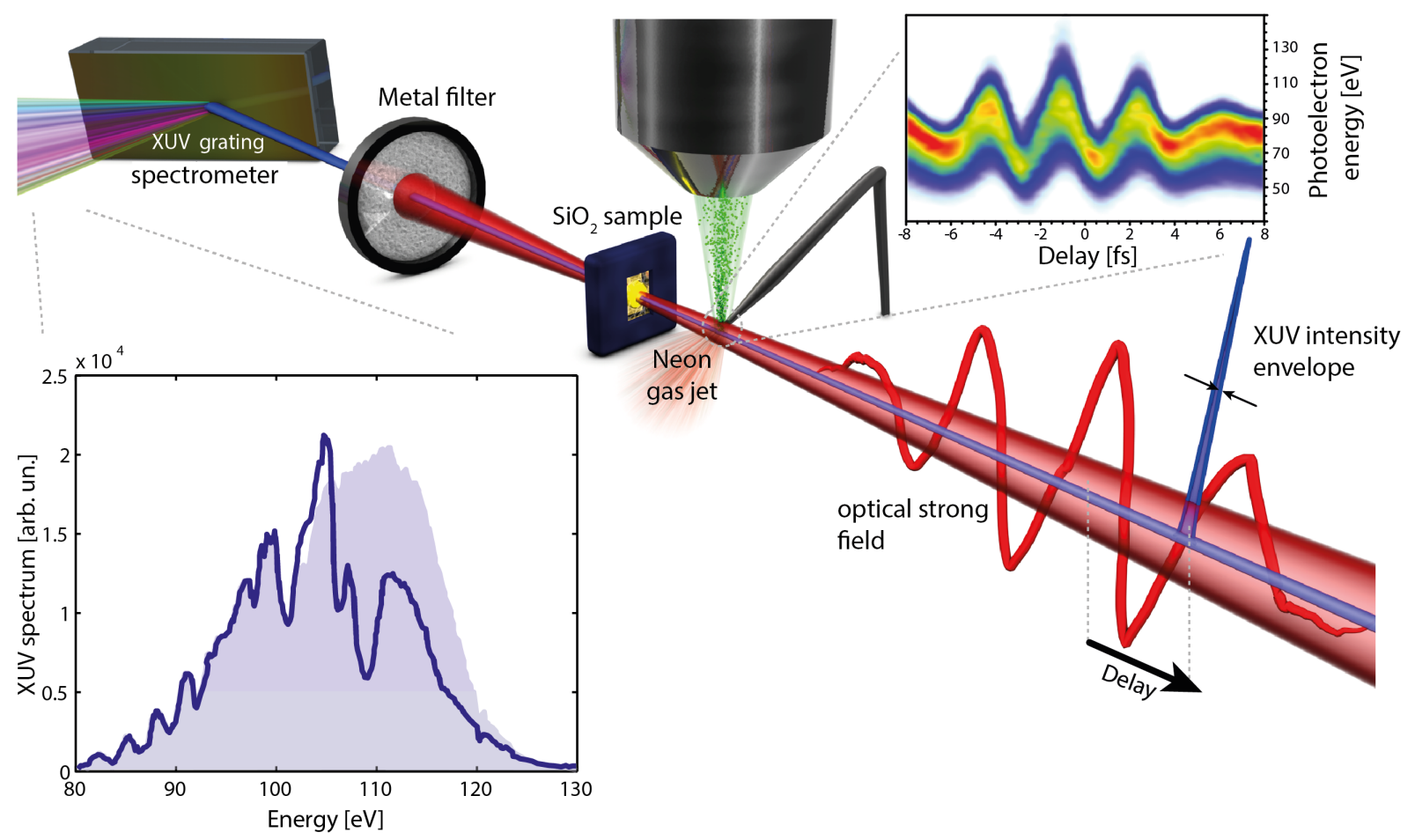

Figure 4.1: Schematic setup for the attosecond time resolved investigation of the XUV absorptivity under the influence of a strong few cycle field (red line).

The field amplitude of the few femtosecond pump pulse at the sample is adjusted to $2.5 \cdot 10^{10} \mathrm{~V} / \mathrm{m}$ close to the breakdown threshold of the material (appendix 9.7). The strong field is transmitted through the fused silica foil with variable delay to the attosecond pulse. In this way the dynamics the pump field induces in the material can be observed with attosecond resolution in the modification of the XUV absorption. To obtain precise knowledge about the absolute timing between the pump and probe pulse, the strong field is additionally temporally characterized with attosecond streaking (appendix 9.4) at the sample. Therefore a nozzle emitting a dilute Neon gas jet is positioned directly in front of the fused silica foil (Fig.(4.1)).

The recorded XUV absorptivity as a function of the delay between the attosecond pulse and the strong field is depicted in Fig.(4.2 B) for the entire energy range between $105-112 \mathrm{eV}$.

For clearer visibility a lineout at $109 \mathrm{eV}$ energy is highlighted in Fig.(4.3B). Panel A shows the electric field of the pump pulse extracted from the streaking measurement. In temporal overlap the XUV absorptivity is reduced up to $10 \%$. Strikingly its amplitude is modulation with twice the laser frequency on sub femtosecond timescales. This means that the dynamics induced in the material are related to the carrier wave of the pump field. The nonlinearity does not only affect the amplitude of the absorptivity but also the energetic position of the absorption line. In correlation to the driving field the conduction band edge is shifted to lower energies (Fig.(4.3 C)). With vanishing pump field the system immediately returns to its original state. 


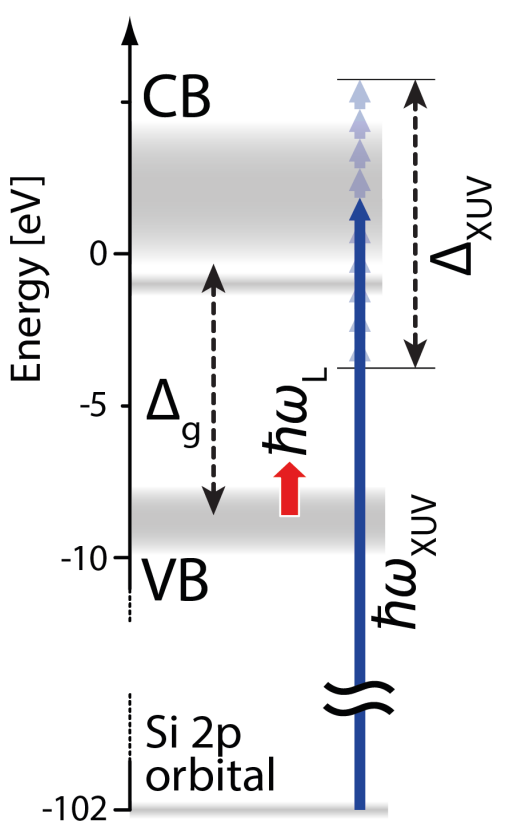

(A)

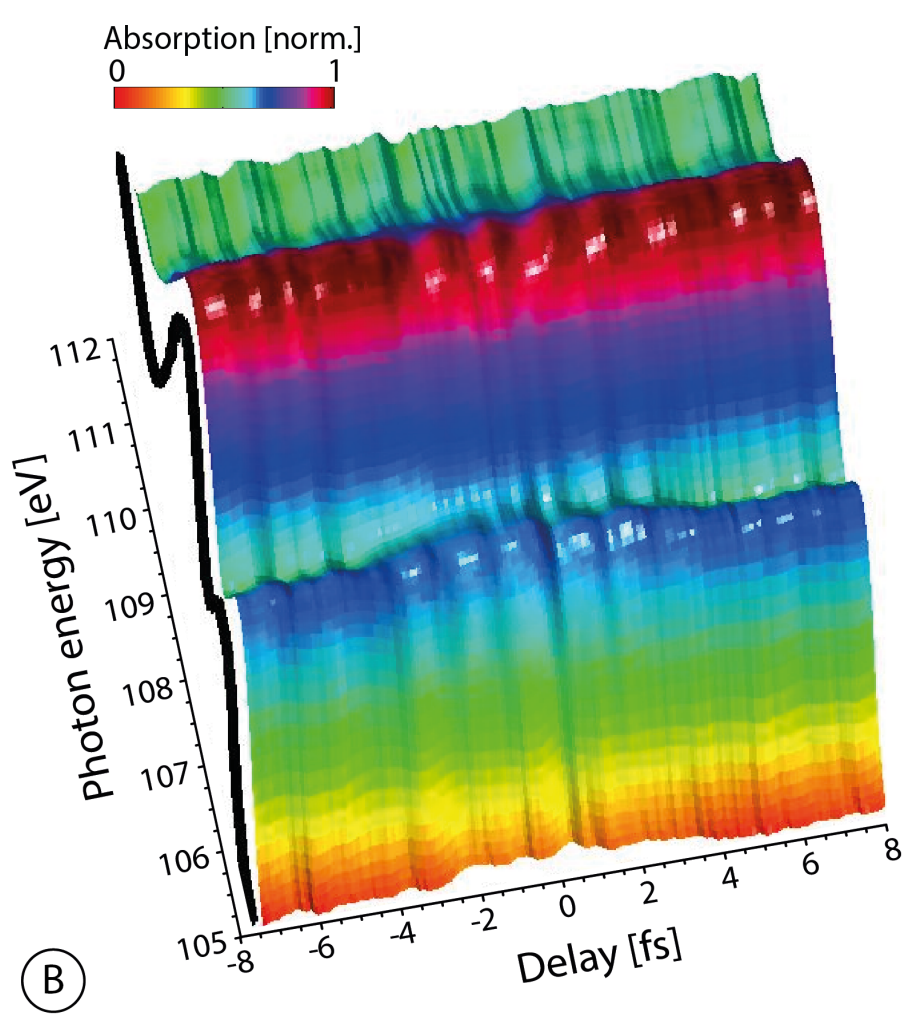

Figure 4.2: Panel A: The attosecond XUV pulses cover a bandwith from $90-120 \mathrm{eV}$ and can excite electrons from inner core states into the conduction band of fused silica. Panel B: The absorptivity of the XUV pulse in the energy range of $105-112 \mathrm{eV}$ as a function of its delay to the strong femtosecond laser field.

This results demonstrate that the material properties of dielectrics can follow electric fields at petahertz frequencies. Even at intensities close to optical breakdown (appendix 9.7) the modification of the electronic structure is entirely reversible and vanishes directly after the pulse. These findings reveal the potential of ultrafast strong field dynamics for future signal processing. They show that it is principally possible to manipulate insulators with few cycle pulses on sub femtosecond timescales. However many of the questions raised in section 1 remain unanswered. To identify the physical origin of the investigated strong field dynamics the understanding of the underlying nonlinear processes has to be extended. 


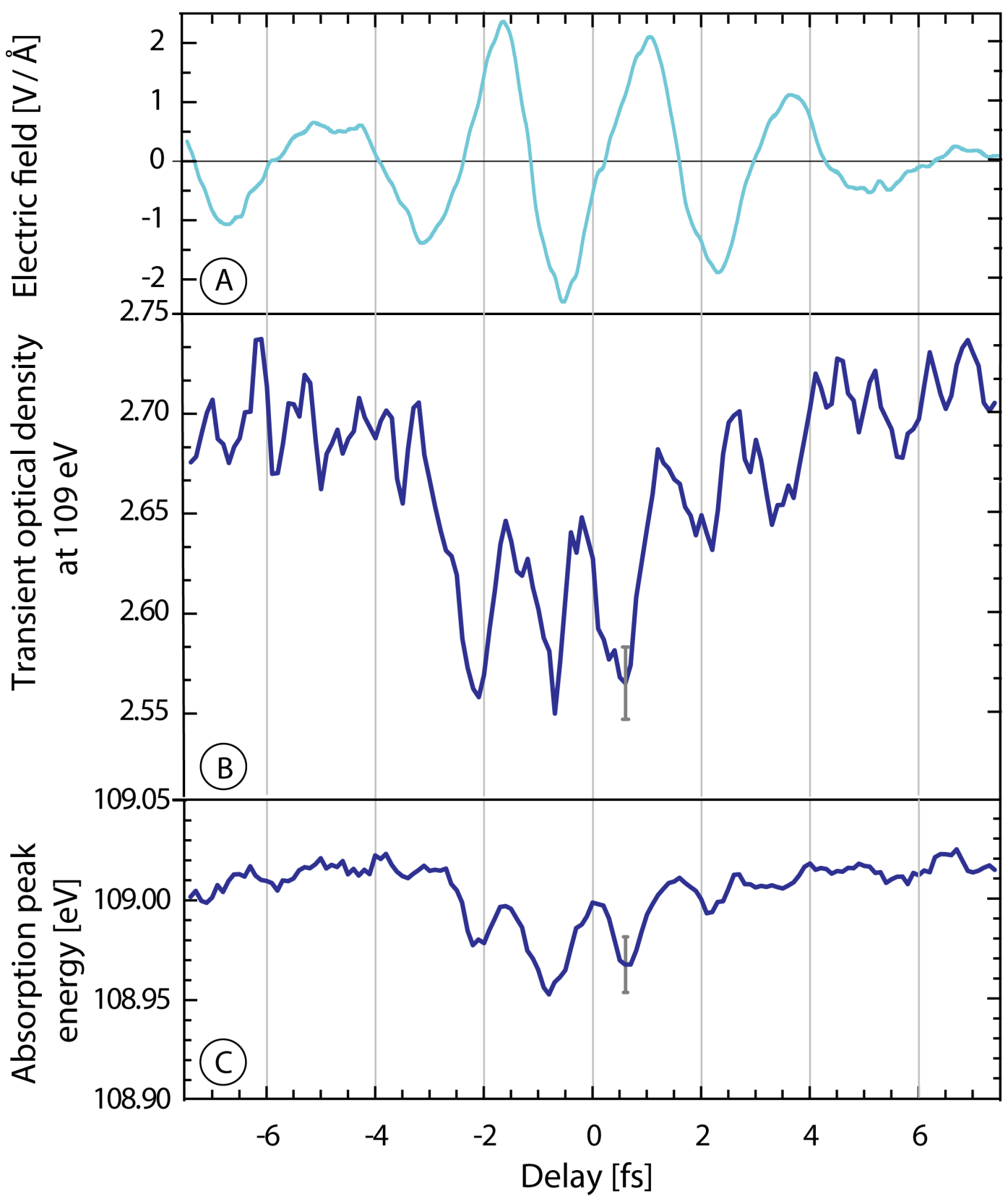

Figure 4.3: Panel A: The electric field of the pump pulse at the target. Panel B: The absorptivity of the XUV pulse as a function of its delay to the strong field is averaged over the energy range between $108.5-109.5 \mathrm{eV}$. Panel C: The energetic position of the XUV absorption line at $108.5-109.5 \mathrm{eV}$ depending on the delay of the pump and probe field. 


\section{Chapter 5}

\section{Strong Field Modification of the Reflectivity}

In the previous chapter a modulation of the XUV absorptivity by up to $10 \%$ in the presence of the strong field is detected. This modification is the signature of a severe field induced perturbation of the electronic system which should also affect other experimental observables. Once the strong field dynamics are triggered they are assumed to affect the penetration of the pump field into the medium. To investigate this phenomena the reflectivity of the few-cycle laser pulse is recorded as a function of its amplitude with femtosecond resolution.

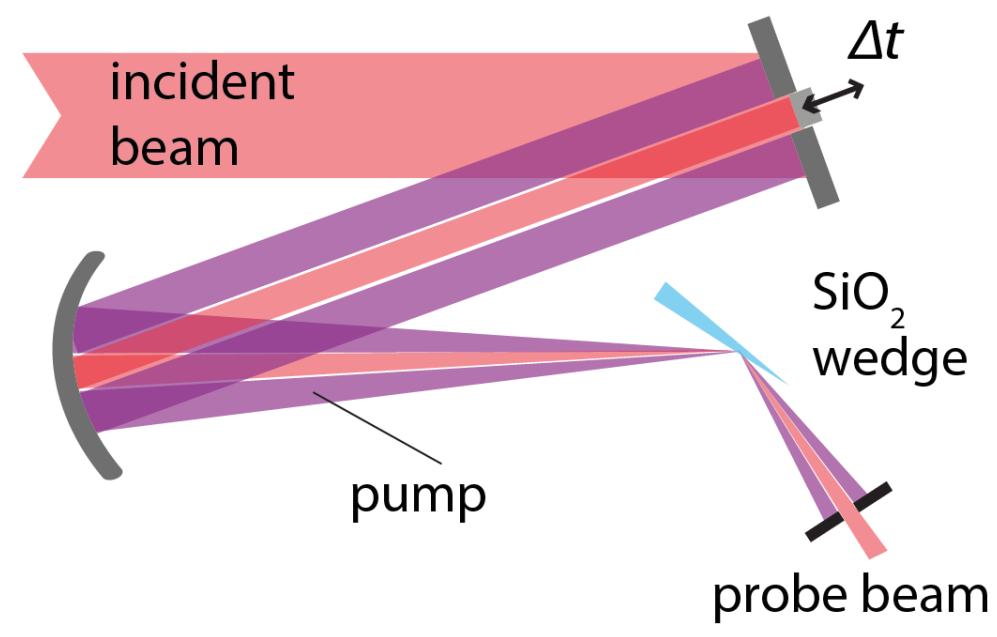

Figure 5.1: Schematic setup for the time resolved investigation of the influence of strong fields on the reflectivity of dielectrics.

To perform this study a two component mirror divides the femtosecond pulse (appendix 9.1.1) into a weak probe and an intense pump beam (Fig.(5.1)). The inner mirror attenuates the pulse intensity by approximately $90 \%$, whereas the outer part possesses almost perfect reflectivity. By moving the segments with respect to each other, a variable delay between the pump and probe pulse can be introduced. The amplitude of the pump field can be 
adjusted with an aperture. Both beams are focused onto the same spot on the surface of a wedged fused silica sample. The target is positioned in Brewster's angle to optimize the signal-to-noise ratio. An additional aperture blocks the reflected pump light and only the reflectivity of the weak probe field is recorded.

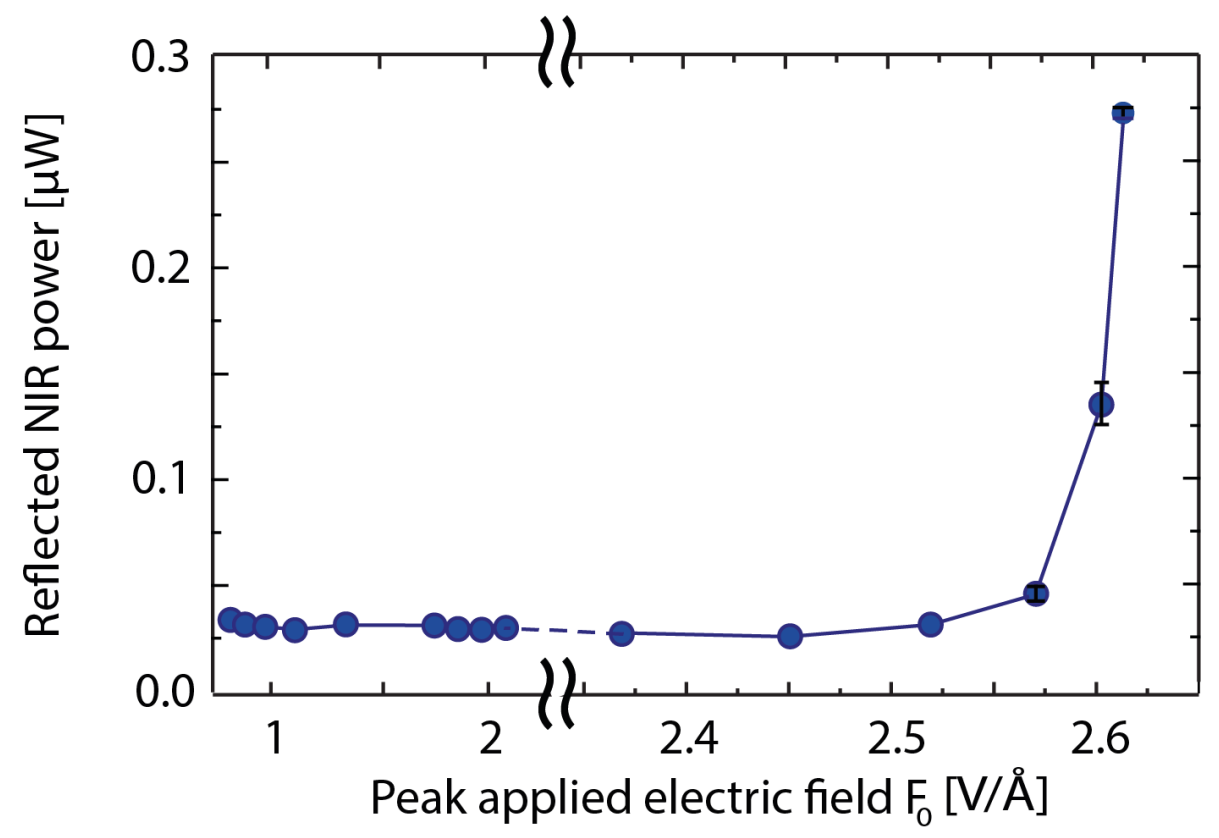

Figure 5.2: The reflectivity of the strong field is measured depending on its intensity.

In a first step the pump and probe pulse are adjusted in temporal overlap and the amplitude of the pump field is steadily increased. When the field strength exceeds $2.5 \mathrm{~V} /$ A the reflectivity of the probe beam rises very nonlinearly. An increase in the field amplitude of $0.1 \mathrm{~V} / \AA$ intensifies the reflected pulse by more than one order of magnitude (Fig.(5.2)). This steep rise cannot be explained with the Kerr effect (Eqn.3.3).

The strong field modification of the XUV absorptivity described in the previous chapter is reversible on femtosecond timescales. To examine whether the detected reflectivity change is related to these fast dynamics it is investigated with temporal resolution. The intensity of the pump field is adjusted to a level where it alone does not alter the probed reflectivity. Only when the pump and probe pulse temporally overlap and interfere constructively at the sample, the field strength is high enough to induce the nonlinearity. The change in the probed reflectivity $S$ is recorded as a function of the delay between the two pulses. The measured autocorrelation curve is displayed in Fig.(5.3). For negative delay the weak probe pulse precedes the pump field and no modification of the reflected signal is detectable. When the two fields start to overlap the nonlinearity is induced. The oscillation period of the electric field is $T_{L}=2.6 \mathrm{fs}$. Comparison of the value of $S\left(-T_{L}\right)$ and $S\left(T_{L}\right)$ shows that the probed reflectivity increases by a factor of $2.1 \pm 0.2$ throughout the interaction. The ratio of $S\left(n \cdot T_{L}\right) / S\left(n \cdot\left(-T_{L}\right)\right)$ for $n= \pm 2, \pm 3, \ldots$ shows no asymmetric behavior. If the lifetime of the induced nonlinearity exceeded several subcycles of the pump field, the assymmetry in 
the autocorrelation would be detectable on longer timescales. This implicates that the system returns to its unperturbed state on femtosecond timescales.

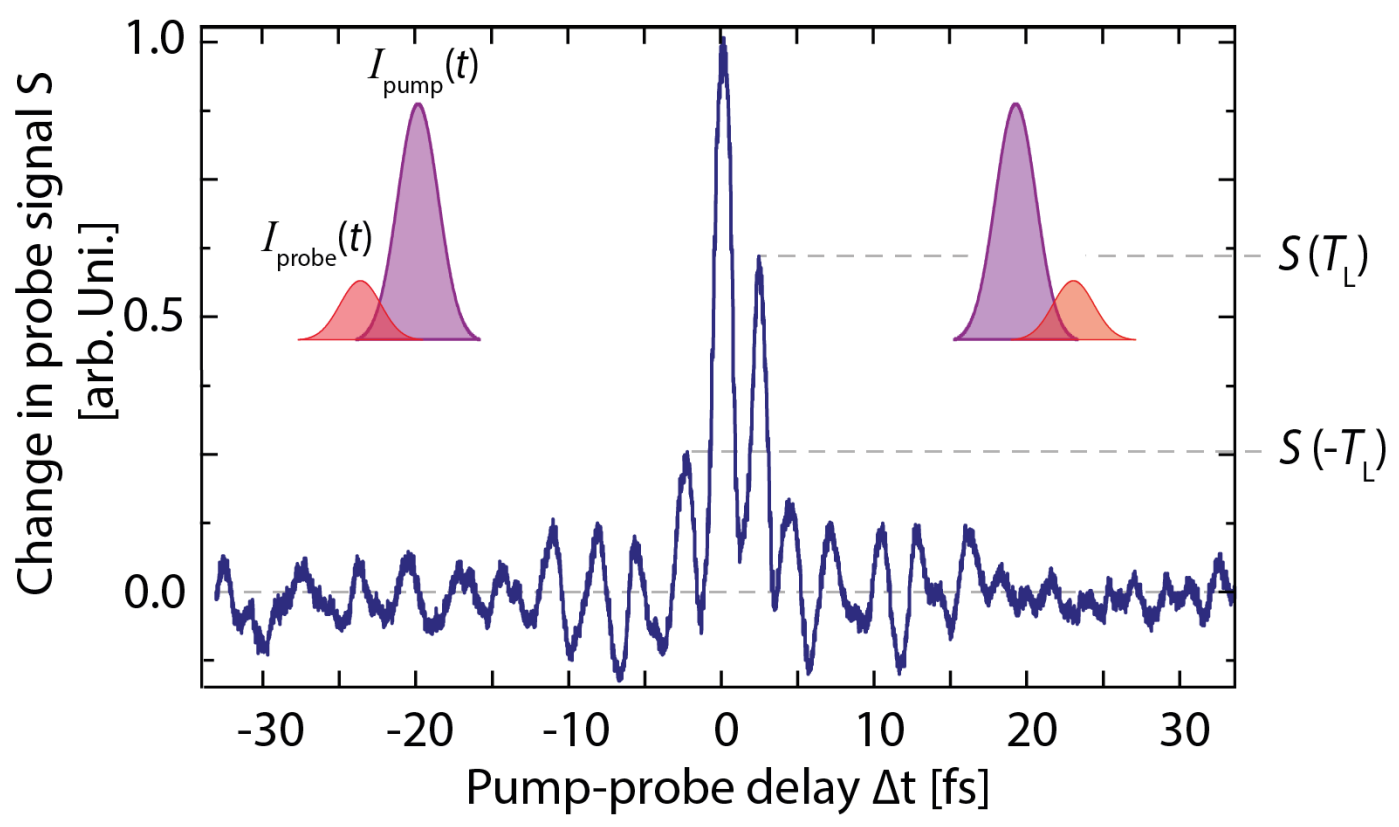

Figure 5.3: The change of the reflected probe light $\mathrm{S}$ is recorded depending on the delay between probe and pump pulse.

The temporal characteristics of the nonlinear increase in reflectivity indicate that it is related to the observed strong field modulation of the XUV absorptivity. This means that intense petahertz fields excite highly nonlinear dynamics evolving on sub-femtosecond scales in dielectrics. The results presented so far can be reproduced by a theoretical model developed by M. Stockmann et al. [29], [47], [48]. The nonlinear polarization predicted by this approach is depicted in Fig.(5.4).

The nonlinear polarization is the interesting quantity to comprehensively understand the dynamics excited in the light-matter interaction. In Terahertz spectroscopy it can be tracked experimentally for pump fields with sub-millimeter wavelength [27, 28, 49, 50]. At optical frequencies $(0.1 \mu \mathrm{m}<\lambda<1 \mu \mathrm{m})$ it is not possible to directly access the induced nonlinear polarization in a time resolved manner so far. It can only be extracted from complex theoretical modeling. As visible in Fig.(5.4) the signal-to-noise ratio of the calculated result is not sufficient to draw reliable conclusions from it.

The presented experimental results describe the influence of the strong field dynamics induced by few-cycle pulses in dieletrics on two different physical observables. The findings provide important information about the temporal evolution and the nonlinear scaling of the excited mechanisms. However both experimental approaches probe individual features of the light-matter interaction. To understand the physical processes triggered by the strong field it is necessary to get a comprehensive picture of the material response and access the nonlinear polarization. In principle the complete signature of the nonlinear interaction is imprinted on the temporal structure of the reflected strong field. In the presented approach it 


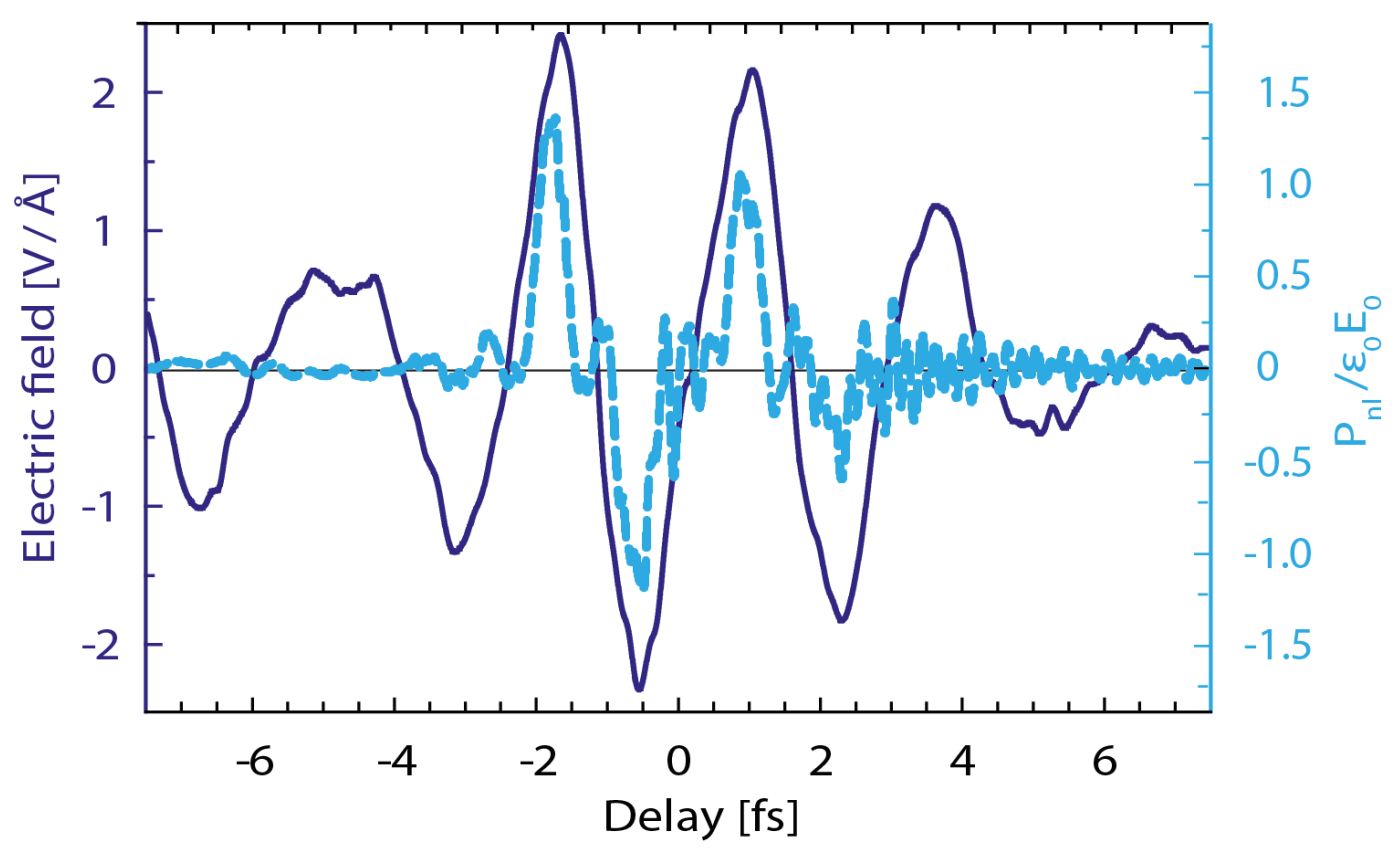

Figure 5.4: Theoretically predicted nonlinear polarization (light blue dashed line) induced by the incident electric field (dark blue line) [29], [47], [48].

can only be resolved with femtosecond resolution. Observation of the reflected pulse in real time would provide complete information about the nonlinear dynamics. The experimental technique described in the following section makes use of this idea. 


\section{Chapter 6}

\section{Attosecond Polarization Spectroscopy}

The aim of this thesis is to examine whether optical few cycle pulses and dielectrics can be used in ultrafast signal processing. The findings presented so far already provide several answers to the questions raised in section 1. Ultrashort pulses can manipulate distinct optical properties of insulators reversibly on femtosecond timescales. The induced dynamics are able to follow the petahertz driving field and exhibit highly nonlinear features. To exploit ultrafast strong field effects for application it is compulsory to understand the physical origin of the dynamics induced in the material. The examination of the modulation of the XUV absorptivity in the presence of the pump field is a very indirect method to investigate the induced changes of the electronic material structure. Examining the reflected strong field, which is modified by the nonlinearity constitutes a more intuitive approach. In the presented implementation the temporal resolution was limited to the femtosecond range.

The light transmitted through the sample delivers complementary information to the reflected radiation. As it is accessible more easily in experiment a novel method is developed which is based on the detection of its nonlinear modification with attosecond time resolution. The measurement technique together with a derived analytical concept provides direct access to the full nonlinear polarization wave emitted by the medium. The knowledge of the nonlinear material response can be used to extract the population and energy transfer dynamics throughout the interaction. So far the dynamics of the nonlinear material response could only be resolved at Terahertz frequencies. Terahertz time domain spectroscopy allows to investigate the temporal profile of the Kerr effect [51-53] and the induced polarization [28, 50]. However its temporal resolution is limited to several tens of femtoseconds at best [27, 49]. The interaction of matter with optical fields evolves on sub femtosecond timescales.

The novel concept is named 'Attosecond Polarization Spectroscopy' (APS). In a first step its applicability is verified in the gas phase. Subsequently it is employed for the comprehensive investigation of the strong field dynamics in dielectrics with attosecond resolution. 


\subsection{Basic Concept}

An external electric field $E(\vec{r}, t)$ entering a material generates oscillating dipoles which themselves emit radiation. The sum over all emitted waves is the polarization $P(\vec{r}, t)$. The propagation of the light wave inside the medium is described by Eqn. 2.6) and can be considered as a polariton consisting of the electric field $E(\vec{r}, t)$ coupled to the polarization wave $P(\vec{r}, t)$. Even though both fields are assumed to be directed along the $\mathrm{x}$-axis of the coordinate system, they are described as plane waves $E(z, t)$ and $P(z, t)$. In a thin sample changes in the transverse field distribution of the propagating field and thus also in the induced polarization are negligible. For this reason $E(z, t)$ and $P(z, t)$ also describe the electric field and polarization on the optical axis of the incident laser beam. Eqn.(2.6) can be solved with a more general approach than presented in section 2 if the electric field is decomposed into fast oscillating complex plane waves modulated with a slowly varying amplitude

$$
\widetilde{E}_{\omega}(z)=\widetilde{A}_{\omega}(z) \exp (i k z)
$$

In this case the first and second spatial derivative can be expressed as

$$
\begin{gathered}
\frac{\partial \widetilde{E}_{\omega}(z)}{\partial z}=\frac{\partial \widetilde{A}_{\omega}(z)}{\partial z} \exp (i k z)+i k \widetilde{A}_{\omega}(z) \exp (i k z) \\
\frac{\partial^{2} \widetilde{E}_{\omega}(z)}{\partial z^{2}}=\underbrace{\frac{\partial^{2} \widetilde{A}_{\omega}(z)}{\partial z^{2}}}_{\approx 0} \exp (i k z)+2 i k \frac{\partial \widetilde{A}_{\omega}(z)}{\partial z} \exp (i k z)-k^{2} \widetilde{A}_{\omega}(z) \exp (i k z) .
\end{gathered}
$$

With the slowly varying amplitude approximation the second spatial derivative in Eqn.6.3) can be neglected and Eqn. 2.6. becomes

$$
\frac{\partial \widetilde{A}_{\omega}(z)}{\partial z}=\frac{i \omega^{2}}{2 \epsilon_{0} c^{2} k} \widetilde{P}_{\omega}^{N L}(z) \exp (-i k z)
$$

which is identical to

$$
\frac{\partial \widetilde{E}_{\omega}(z)}{\partial z}=i k \widetilde{E}_{\omega}(z)+\frac{i \omega}{2 \epsilon_{0} c n} \widetilde{P}_{\omega}^{N L}(z)
$$

Eqn. (6.5) is generally solved by

$$
\widetilde{E}_{\omega}(z)=\widetilde{E}_{\omega}(0) \exp (i k z)-\frac{i \omega \exp (i k z)}{2 \epsilon_{0} n c} \int_{0}^{z} \exp \left(-\left(i k z^{\prime}\right)\right) \widetilde{P}_{\omega}^{N L}\left(z^{\prime}\right) d z^{\prime} .
$$

As expected in the absence of a nonlinear material response, Eqn.(6.6) is the plane wave solution $\widetilde{E}_{\omega}(z)=\widetilde{E}_{\omega}(0) \exp (i k z)$ with the amplitude $\widetilde{E}_{\omega}(0)$ of the incident wave at the 
entrance of the medium. If the interaction length, which is identical to the material thickness is small the nonlinear modification of the incident field during propagation is negligible. Therefore all dipoles can be assumed to be excited by the same waveform and oscillate in phase with $E(z, t)$

$$
\widetilde{P}_{\omega}^{N L}(z)=\widetilde{p}_{\omega} \exp (i k z)
$$

With this ansatz Eqn.(6.6) becomes

$$
\widetilde{E}_{\omega}(z)-\widetilde{E}_{\omega}(0) \exp (i k z)=-\frac{i \omega z}{2 \epsilon_{0} n c} \widetilde{P}_{\omega}^{N L}(z) .
$$

$\widetilde{E}_{\omega}^{l i n}(z)=\widetilde{E}_{\omega}(0) \exp (i k z)$ is the linear propagated incident field. Eqn. 6.8$)$ shows that the assumption of phase matched propagation of the driving field and the nonlinear polarization (Eqn.(6.7) ) means, that the modification of the waveform due to the nonlinear source term grows linearly with propagation distance. The validity of this approximation is experimentally verified in section 6.4.6.

The polarization source term can be written as

$$
\widetilde{P}_{\omega}^{N L}(z)=\frac{2 i n \epsilon_{0} c}{\omega z}\left[\widetilde{E}_{\omega}(z)-\widetilde{E}_{\omega}^{l i n}(z)\right] .
$$

As the phase of $\widetilde{P}_{\omega}^{N L}(z)$ evolves linearly the nonlinear polarization can be calculated from the fields $\widetilde{E}_{\omega}^{\operatorname{lin}}(\ell)$ and $\widetilde{E}_{\omega}(\ell)$ propagated through the material with thickness $\ell$

$$
\widetilde{P}_{\omega}^{N L}(z)=\frac{2 i n \epsilon_{0} c}{\omega \ell} \Delta \widetilde{E}_{\omega} \exp (i k(z-\ell))
$$

where

$$
\Delta \widetilde{E}_{\omega}=\widetilde{E}_{\omega}(\ell)-\widetilde{E}_{\omega}^{l i n}(\ell) .
$$

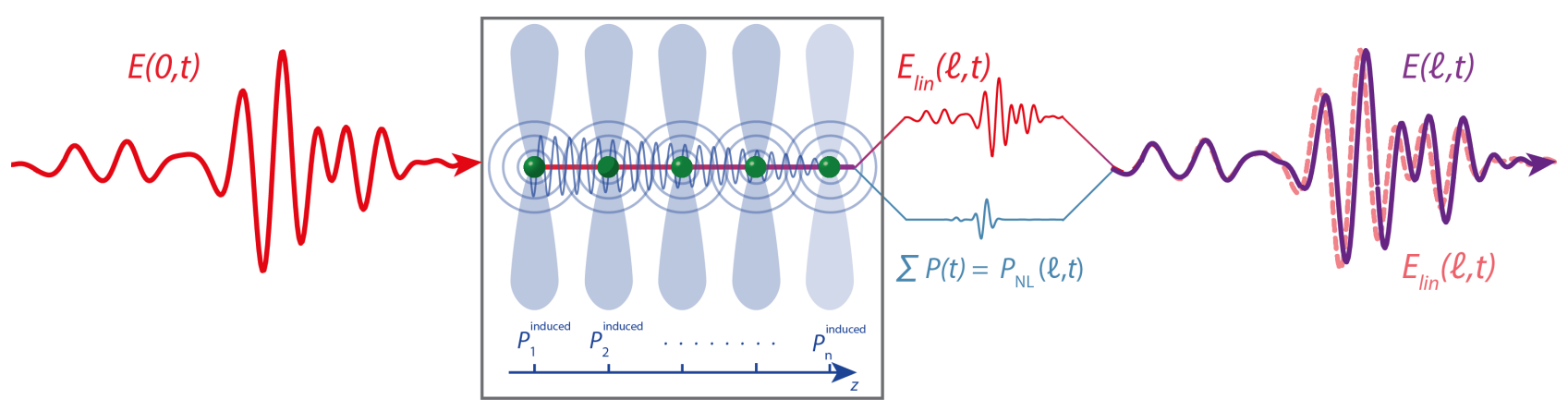

Figure 6.1: Illustration of the physical concept of the nonlinear light-matter interaction exploited for Attosecond Polarization Spectroscopy.

Fig.(6.1) illustrates the meaning of Eqn.(6.10) in time: If the electric field propagates through a medium it excites electric dipoles which emit a polarization wave. Depending on the amplitude of the incident field $E(0, t)$, the electronic properties follow the driving force 
linearly or for high field strength develop a nonlinear contribution. According to Eqn.(6.8) the nonlinearly modified wave exciting the material $E(\ell, t)$ is the sum of the linear propagated field $E_{l i n}(\ell, t)$ and the induced nonlinear polarization wave $P_{N L}(\ell, t)$. This means that the nonlinear polarization is accessible via the difference between $E(\ell, t)$ and $E_{l i n}(\ell, t)$ after propagation through the sample (Eqn.(6.10)).
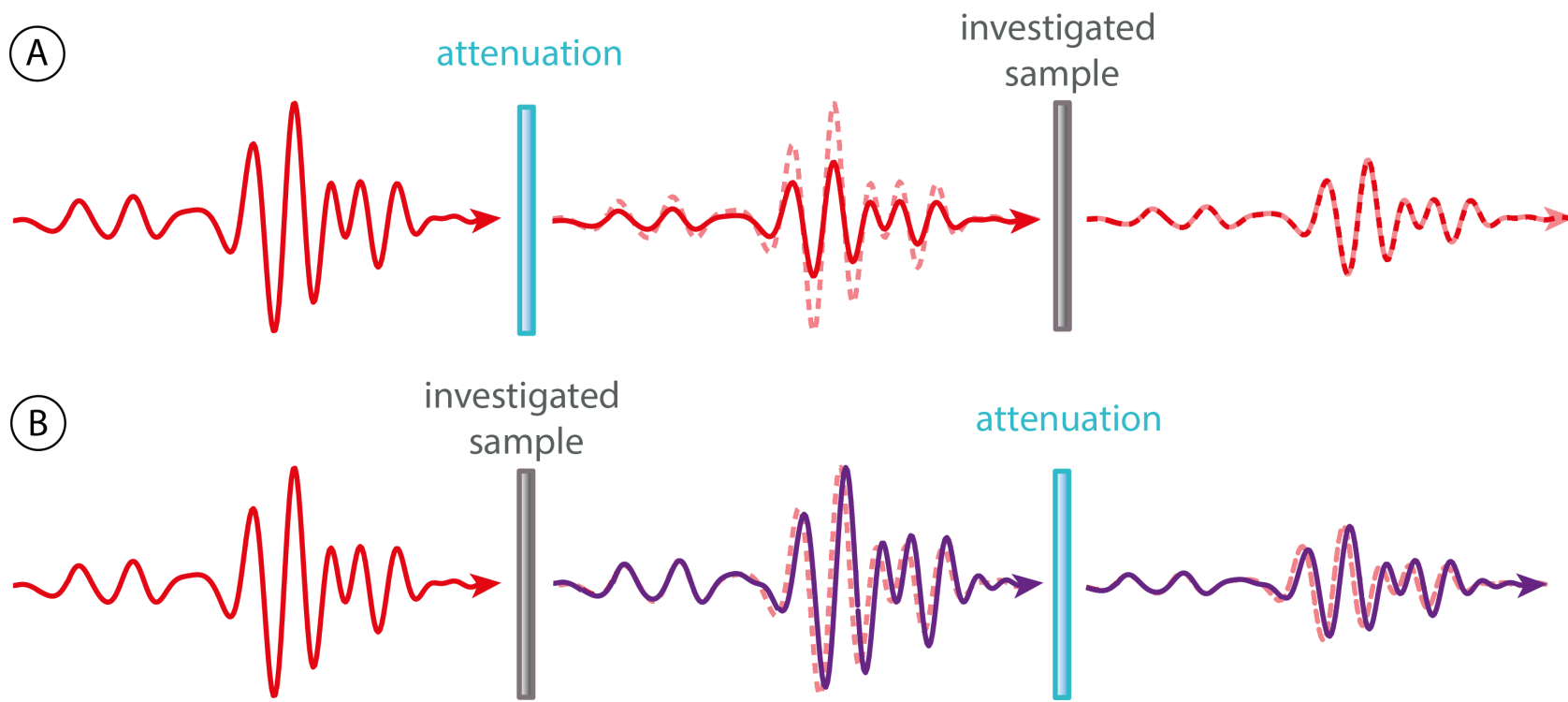

Figure 6.2: Schematic principle of Attosecond Polarization Spectroscopy. Panel A: To access $E_{r e f}(\ell, t)$ the pulse is attenuated before it passes through the material. In the interaction it is only affected by the linear dispersion of the medium. Panel B: The strong field $E(\ell, t)$ excites a nonlinear material response. After the interaction the amplitudes of the reference and the strong field are theoretically matched.

Attosecond Polarization Spectroscopy makes use of Eqn.(6.10) to access the nonlinear polarization induced in matter by intense optical few-cycle fields. Its experimental implementation is schematically illustrated in Fig. (6.2). To record $E(\ell, t)$ and $E_{l i n}(\ell, t)$ two measurements are performed. For $E_{\text {lin }}(\ell, t)$ the amplitude of the field is decreased with a frequency independent attenuation factor $\beta<<1$ before the pulse is transmitted through the material (Panel A). $\beta$ is chosen so small that the weak reference field $E_{r e f}(z, t)=\beta E(z, t)$ only interacts linearly with the medium and a negligibly small nonlinear polarization wave is induced. To determine $E(\ell, t)$ the field is transmitted through the material at a field strength close to optical breakdown (Panel B) and excites a nonlinear material response. In both cases the pulses are modified by linear dispersion. The characteristics of the nonlinear polarization are only imprinted on the intense field $E(\ell, t)$. To compare $E_{r e f}(z, t)$ and $E(\ell, t)$ the attenuation of $E_{r e f}(z, t)$ is theoretically compensated for after the interaction. With Eqn.(6.10) the Fourier transforms (Eqn.(2.7)) of $E_{\text {lin }}(\ell, t)=\beta^{-1} E_{r e f}(\ell, t)$ and $E(\ell, t)$ provide direct access to the nonlinear polarization induced in the material.

Eqn. 6.10 is a general relation suited to calculate $\widetilde{P}_{\omega}^{N L}(z)$ at every z position in the medium, however the accuracy of the obtained result differs with propagation distance. In the ansatz 
in Eqn. 6.7) the amplitude of the polarization wave $\tilde{p}_{\omega}$ is approximated with the 0th order contribution of the Taylor expansion of $\widetilde{p}_{\omega}(z)$

$$
\widetilde{p}_{\omega}(z)=\widetilde{p}_{\omega}+{\frac{\partial \widetilde{p}_{\omega}(z)}{\partial z}}_{z=\ell / 2}(z-\ell / 2)+. .
$$

As the next higher order term increases linearly with $z$ the leading error for $\widetilde{p}_{\omega}(z)$ vanishes at the midpoint of the material $z=\ell / 2$. Therefore in the course of this thesis $\widetilde{P}_{\omega}^{N L}(\ell / 2)$ will always be evaluated at the middle of the medium. In the experiment the fields can only be measured after exciting the material. Therefore $E_{r e f}(\ell / 2, t)$ and $E(\ell / 2, t)$ are not directly accessible experimentally. To obtain $E_{r e f}(\ell / 2, t)$ the linear dispersion properties of the measured $E_{r e f}(\ell, t)$ are back propagated in frequency space to the midpoint of the material $z=\ell / 2$

$$
\widetilde{E}_{\omega}^{r e f}(\ell / 2)=\widetilde{E}_{\omega}^{r e f}(\ell) \exp \left(-i \frac{\omega n \ell}{2 c}\right) .
$$

The same is performed with $E(\ell, t)$

$$
\widetilde{E}_{\omega}^{h i g h}(\ell / 2)=\widetilde{E}_{\omega}(\ell) \exp \left(-i \frac{\omega n \ell}{2 c}\right) .
$$

As the nonlinear modification of the strong field increases linearly with propagation (Eqn.(6.8)), $\widetilde{E}_{\omega}(\ell / 2)$ is taken as the average of $\beta^{-1} \widetilde{E}_{\omega}^{r e f}\left(\ell / 2\right.$ and $\widetilde{E}_{\omega}^{h i g h}(\ell / 2)$

$$
\widetilde{E}_{\omega}(\ell / 2)=\frac{1}{2}\left(\beta^{-1} \widetilde{E}_{\omega}^{r e f}(\ell / 2)+\widetilde{E}_{\omega}^{h i g h}(\ell / 2)\right) .
$$

The knowledge of the temporal evolution of the electric field and the induced nonlinear polarization provides access to the time resolved energy density $W(z, t)$ transferred between field and material. As the polarization wave can be determined with the best accuracy at the midpoint of the material $W(\ell / 2, t)$ is also evaluated for this position

$$
W(\ell / 2, t)=\int_{-\infty}^{t} E\left(\ell / 2, t^{\prime}\right) \frac{d P\left(\ell / 2, t^{\prime}\right)}{d t^{\prime}} d t^{\prime} .
$$

Its resultant value at the end of the pulse $W(\ell / 2, t \rightarrow \infty)$ determines the total amount of energy dissipated in the interaction.

\subsection{Experimental Implementation}

The experimental setup of Attosecond Polarization Spectroscopy is sketched in Fig. 6.4). The measurement is performed with intense optical few-cycle laser pulses with a duration of $\tau<4 f_{S}$ (Fig. 6.4), appendix 9.1.1). In a first step these pulses are employed to generate 


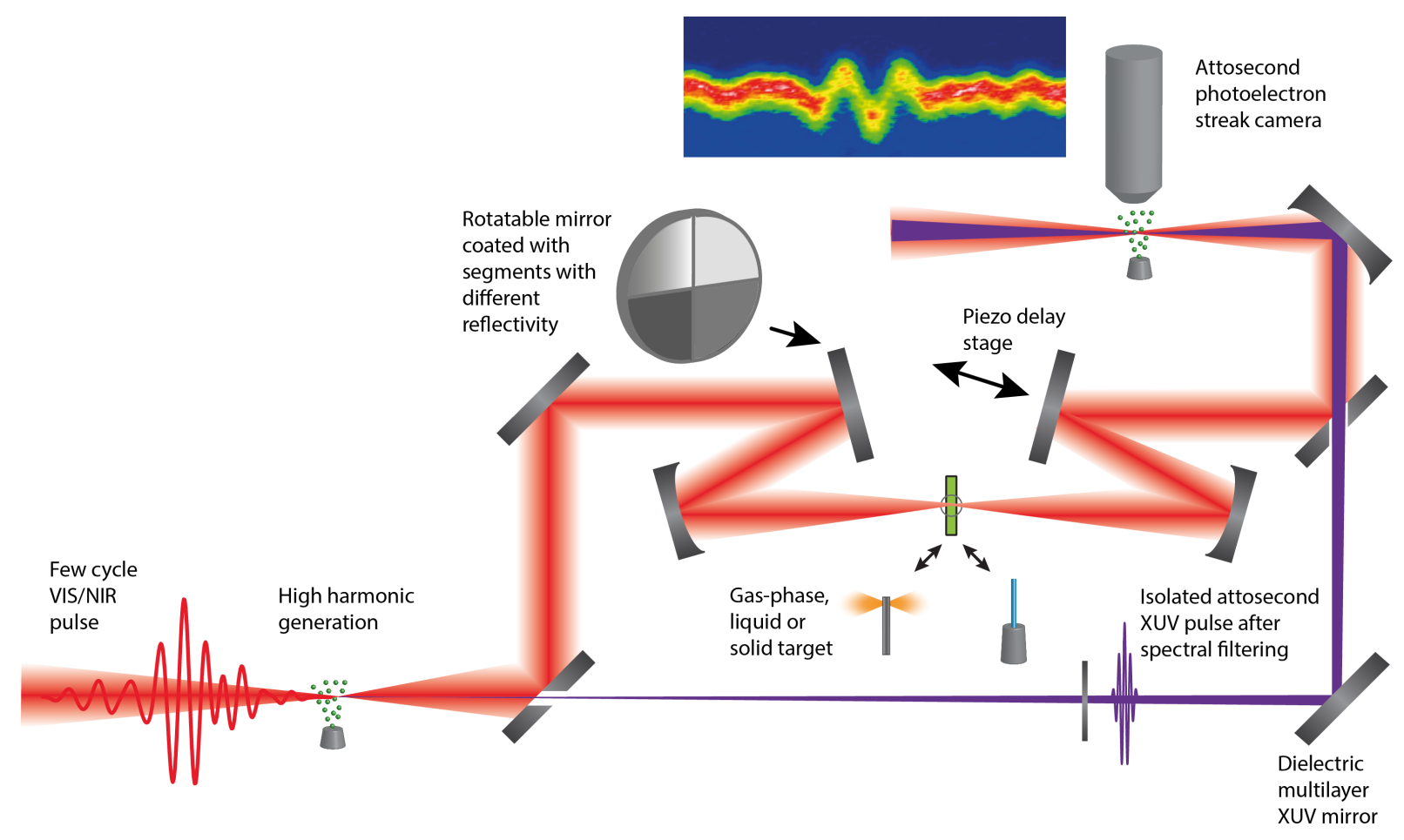

Figure 6.3: Schematical experimental setup for Attosecond Polarization Spectroscopy

extreme ultraviolet light bursts with less than 200 as duration (appendix 9.3). Subsequently the two beams are spatially separated with a perforated mirror and launched into the arms of a Michelson interferometer [54]. There the few-cycle pulse is focused onto the target under scrutiny. The Rayleigh length [38] is adjusted to considerably exceed the thickness of the investigated samples. To record $E_{r e f}(\ell, t)$ and $E(\ell, t)$ the intensity on the target has to be varied to switch from the linear to the nonlinear interaction regime. This is realized in a dispersion free manner by reflecting the beam off a high precision rotatable mirror coated with segments with different degrees of attenuation. This facilitates the variation of the pulse intensity by up to a factor of 15 without changing the temporal or spatial properties of the field and keeping the experimental conditions identical. After transmission through the sample the few-cycle pulse and the XUV radiation are spatially recombined by a second perforated mirror. Both beams are focused onto a dilute Neon gas jet into an attosecond streak camera (appendix 9.4] [55]. The focus at the target under scrutiny is directly imaged into the streak camera where the transmitted field is temporally characterized with attosecond resolution (Fig.(6.4)).

For the APS experiment three independent traces are recorded. To access the linear propagated reference field $E_{r e f 1}(\ell, t)$, the pulse intensity is reduced to a level where only a negligible nonlinear material response is induced. Then the mirror is rotated to a high reflecting segment to measure $E(\ell, t)$. All other experimental conditions remain unchanged. $E_{r e f 1}(\ell, t)$ and $E(\ell, t)$ already provide the complete information to determine the nonlinear 


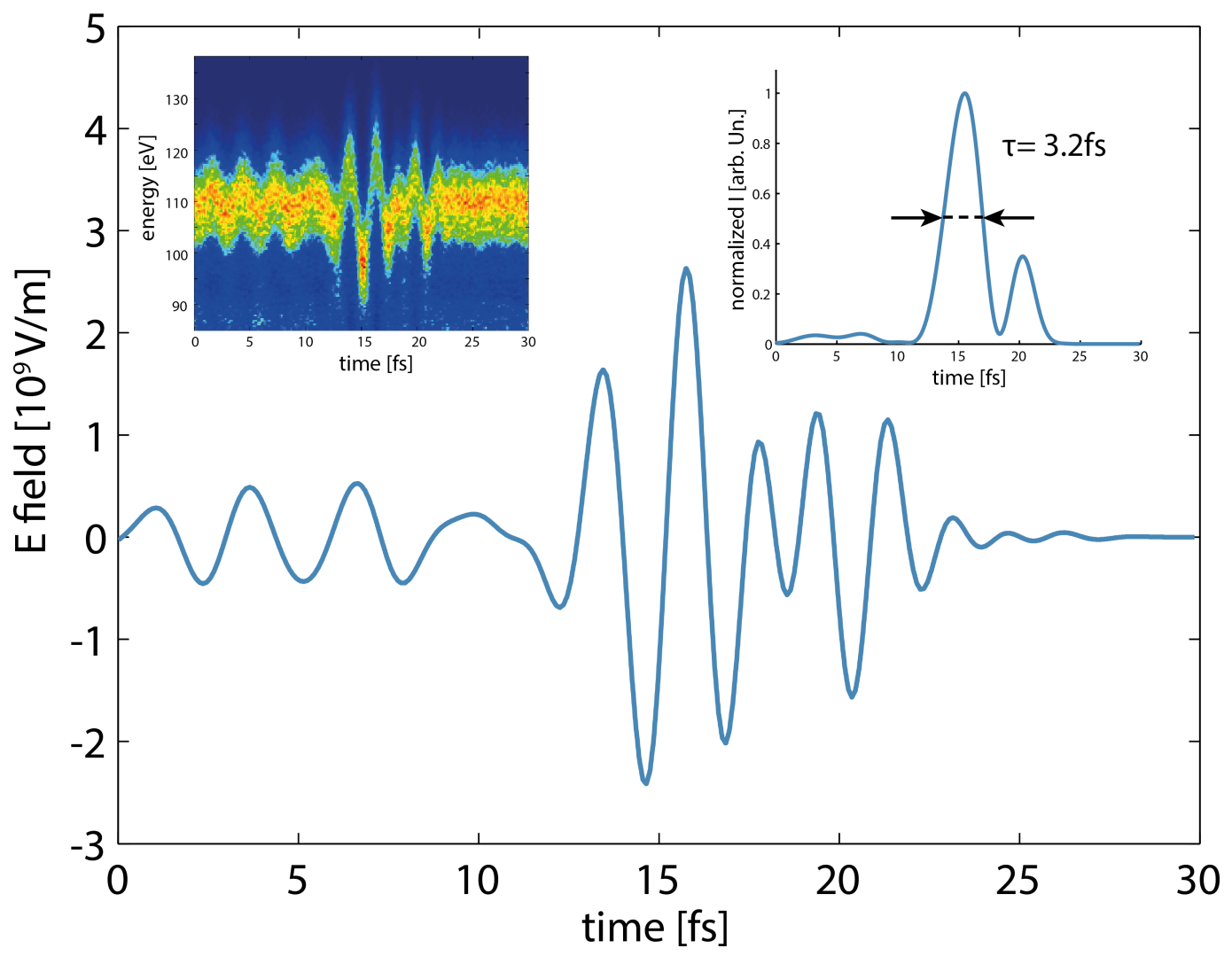

Figure 6.4: The electric field of the optical few-cycle laser pulse with a pulse duration of less than $4 f S$ (evaluated as the temporal full width half maximum of the intensity envelope, right inset) employed for the solid state experiment (appendix 9.1.1). The recorded streaking trace from which the electric field is extracted is depicted in the left inset.

polarization. As the concept of APS is based on the comparison of different scans the stability of the measurement apparatus is very important. To ensure its reliability the reference measurement $E_{r e f 2}(\ell, t)$ is repeated and only scan sets with a timing difference of less than $<30 a$ as between $E_{r e f 1}(\ell, t)$ and $E_{r e f 2}(\ell, t)$ are considered.

\subsection{Verification in the Gas Phase}

If an external field is strong enough to ionize atoms in a gas, the generated plasma reduces the refractive index of the medium (section 2.6.3). To verify the applicability of the new experimental technique, APS is used to investigate the nonlinear interaction of intense fewcycle laser pulses with Neon gas. The aim is to observe the predicted decrease of the optical density of the medium due to plasma excitation. 
The field strength necessary to ionize Neon atoms is significantly higher than the damage threshold (appendix 9.7) of dielectrics. Therefore it is not possible to perform the measurement in the gas phase with the same pulses used for the solid state experiment (Fig.(6.4)). Instead a sub 4 fs pulse with a higher pulse energy is employed (appendix 9.1.2). Its electric field is displayed in Fig.(6.5). It is used to generate XUV pulses with less than 250 as duration (appendix 9.3 .

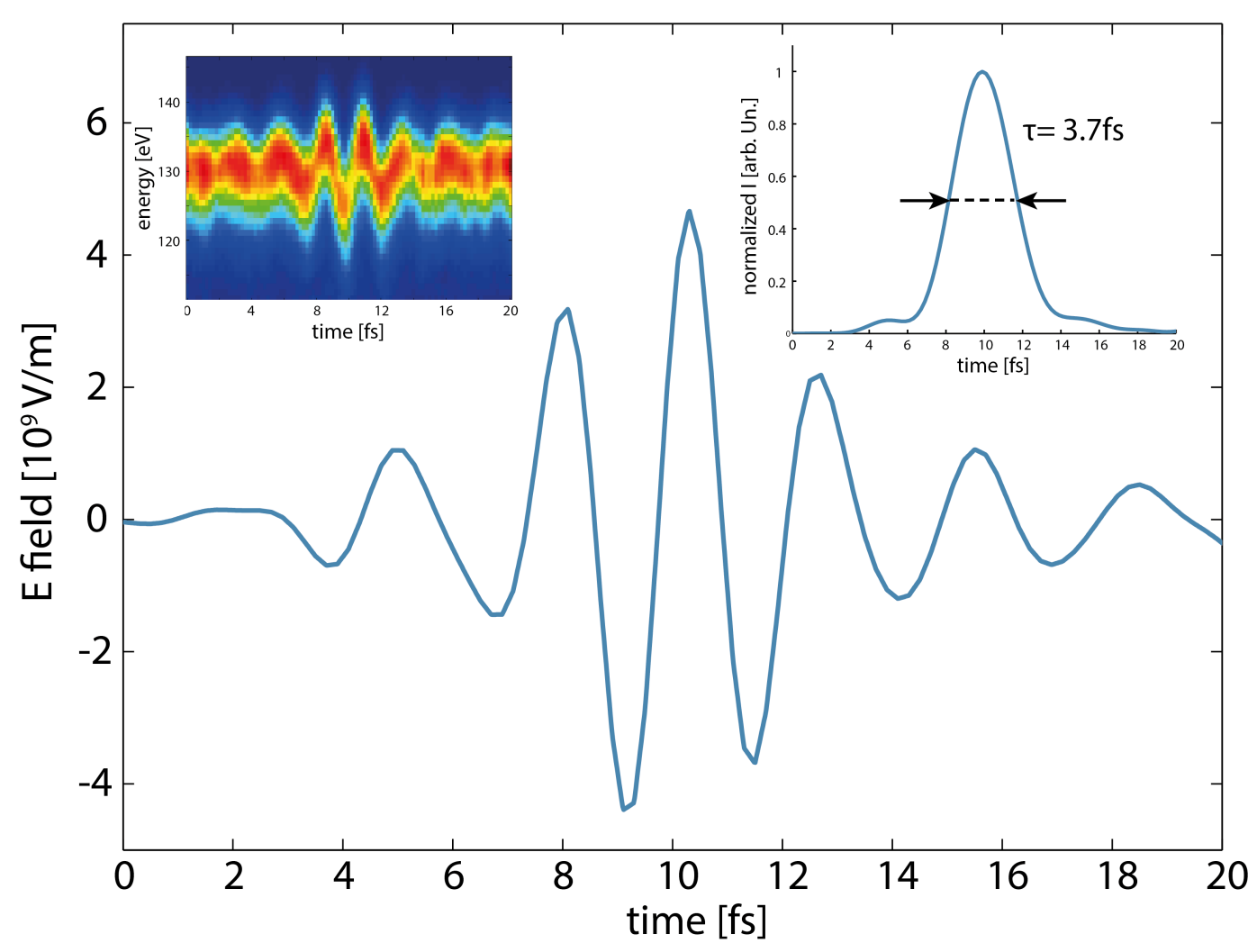

Figure 6.5: The electric field of the VIS/NIR few-cycle laser pulse (appendix 9.1.2) used for the APS measurement in the gas phase.

For the reference measurement the intensity of the femtosecond pulse is adjusted to $I_{r e f}=(1.1 \pm 0.1) \cdot 10^{14} \frac{\mathrm{W}}{\mathrm{cm}^{2}}$. This corresponds to a field strength of $E_{r e f}=(2.8 \pm 0.1) \cdot 10^{10} \frac{\mathrm{V}}{\mathrm{m}}$ $\left(\beta^{-1}=2.5\right)$ and is assumed to be located below the ionization threshold of the nobel gas. $E_{r e f}(\ell, t)$ is compared to the strong field transmitted through the gas cell at an intensity of $I=(6.6 \pm 0.6) \cdot 10^{14} \frac{\mathrm{W}}{\mathrm{cm}^{2}}$. This corresponds to a field amplitude of $E=(7.1 \pm 0.2) \cdot 10^{10} \frac{\mathrm{V}}{\mathrm{m}}$ for which considerable plasma generation is expected.

Keldysh theory [56] describes the impact of the ionization potential $I_{p}$ and the properties of the electric field on the probability for nonlinear photoionization. It identifies multiphoton and tunneling ionization as the two limiting regimes of the nonlinear process. The Keldysh 
parameter $\gamma$ is defined to distinguish between the two ranges.

$$
\begin{gathered}
\gamma=\sqrt{\frac{I_{p}}{2 U_{p}}} \\
U_{p}=\frac{e E^{2}}{4 m \omega_{0}^{2}}
\end{gathered}
$$

$U_{p}$ is the ponderomotive energy and describes the energy a free electron acquires while oscillating in an electric field. Under the assumption that $\hbar \omega_{0}<I_{p}$, the Keldysh parameter $\gamma<1$ for large field amplitudes $E$ and low frequencies $\omega_{0}$ in the strong field regime. For small field amplitudes and high frequencies $\gamma>1$ in the regime of multiphoton ionization. As the ionization potential of Neon is $I_{p}=21.56 \mathrm{eV}$ [57] the Keldysh parameter for the strong field in the APS experiment can be calculated to $\gamma=0.53$. Hence the electron wavepackets escape the Coulomb potential predominately via tunneling ionization [56].

The comparison between the weak reference and the strong field after the propagation through a $1 \mathrm{~mm}$ long gas cell filled with $330 \pm 30$ mbar Neon is depicted in Fig.(6.6). Strikingly, $E(\ell, t)$ (red line in Fig.(6.6 A)) experiences a continuous negative shift to earlier times compared to $E_{r e f}(\ell, t)$ (blue dashed line) during propagation. The carrier wave is accelerated throughout the entire interaction. The shift does not recover at the end of the pulse as the electric field vanishes. This behavior is even better visible in the temporal evolution of the nonlinear phase difference $\Delta \phi_{N L}(t)$ between $E_{r e f}(\ell, t)$ and $E(\ell, t)$ (Fig.(6.6 B)). The phase $\phi(t)$ is extracted from a Hilbert transformation [58] performed for the reference and the strong field. Throughout the pulse $\Delta \phi_{N L}(t)$ steadily decreases to a maximum value of $\Delta \phi_{N L}(15 f s)=\Delta \phi_{N L \max }=-1.1 \pm 0.1 \mathrm{rad}$. Due to the vanishing amplitude of the light field the nonlinear phase shift cannot be reliably evaluated for bigger delays. The strong field is distorted too heavily by the growing dispersion of the medium.

The increasing blue shift of the phase velocity of $E(\ell, t)$ compared to $E_{r e f}(\ell, t)$ originates from the negative plasma contribution to the refractive index described in section 2.6.1. As the Coulomb barrier of the atomic potential well is suppressed by the strong external field the carriers can escape by tunneling. The freed electrons are accelerated by the laser field. Both processes contribute to the nonlinear polarization wave generated in the medium. The coupling between the electric field and the polarization determines the propagation of light inside the gas and makes it travel faster than the linear propagating incident field $E_{r e f}(\ell, t)$. This phenomena is known as photon acceleration [59].

As the lifetime of the plasma exceeds the pulse duration only a small amount of electrons return to their parent ions during the light-matter interaction. This explains the irreversible phase shift $\Delta \phi_{N L \max }=-1.1 \pm 0.1 \mathrm{rad}$ at the end of the pulse which can be related to a change in refractive index $\Delta n$

$$
\Delta n=\frac{\Delta \phi_{N L \max } \lambda}{2 \pi \ell p}
$$




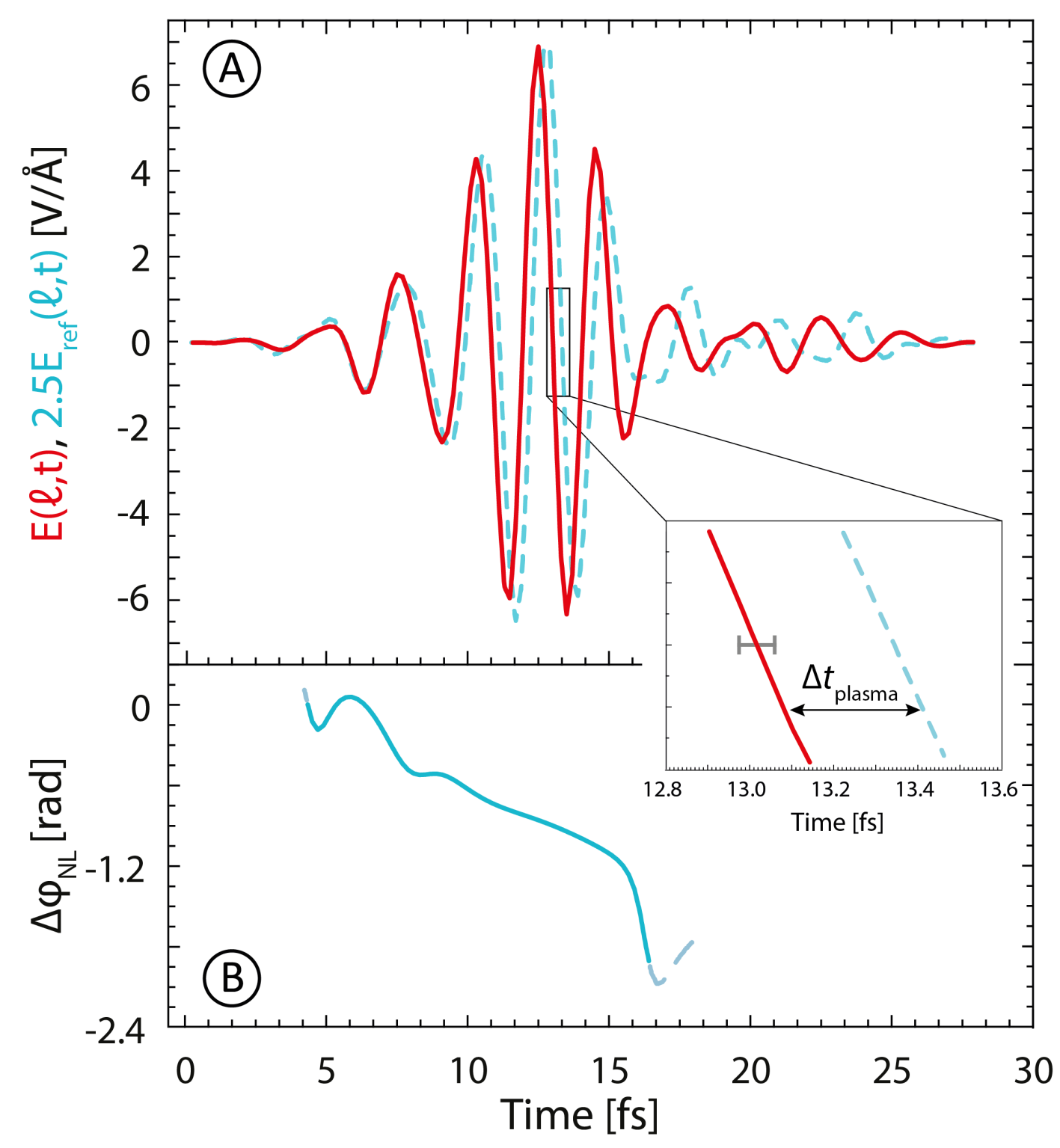

Figure 6.6: Panel A: Comparison of the weak reference wave (blue dashed line) with a field strength of $E_{r e f}=(2.8 \pm 0.1) \cdot 10^{10} \frac{\mathrm{V}}{\mathrm{m}}\left(\beta^{-1}=2.5\right)$ to the strong field $E(\ell, t)=(7.1 \pm 0.2)$. $10^{10} \frac{\mathrm{V}}{\mathrm{m}}$ (red line) after the interaction with Neon gas. $E(\ell, t)$ is assumed to ionize a significant fraction of the irradiated Neon atoms. The generated plasma reduces the refractive index of the medium. Therefore during propagation $E(\ell, t)$ experiences an increasing negative shift (highlighted in the inset) compared to $E_{r e f}(\ell, t)$. This is explicitly visible in the difference in phase between $E_{r e f}(\ell, t)$ and $E(\ell, t)$ in Panel B. 
With $\lambda=780 \mathrm{~nm}$, the interaction length $\ell=1 \mathrm{~mm}$ and the pressure $p=330 \pm 30 \mathrm{mbar}$ in the gas cell, $\Delta n$ is calculated to $\Delta n=(-4.1 \pm 0.1) \cdot 10^{-4}$. The refractive index of neutral Neon atoms is $n_{\text {Neon }} \approx 1$ at optical frequencies. According to Eqn.(2.43) the refractive index change $\Delta n$ is caused by a free electron density of $n_{e}=(1.4 \pm 0.1) \cdot 10^{18} \frac{1}{\mathrm{~cm}^{3}}$. Compared to the atomic density of the neutral gas $\left(n_{\text {Neon }}=8.4 \cdot 10^{18} \frac{1}{\mathrm{~cm}^{3}}\right.$ at $\left.330 \mathrm{mbar}\right)$, this corresponds to a fraction of $(17 \pm 1.5) \%$ atoms ionized in the light-matter interaction.

According to Eqn.(2.50) two physical processes contribute to the nonlinear polarization response of the plasma: on the one hand the escape of bound electrons from the atomic potential, on the other hand the acceleration of the freed carriers in the electric field. As the tunneling rate increases with field strength the probability for ionization is highest at the peaks of the electric field in each half cycle. This dependency should cause a steep increase of the absolute value of $\Delta \phi_{N L}(t)$ at each local field extremum. The lack of steps in the quasi constant decrease of $\Delta \phi_{N L}(t)$ (Fig.(6.6 B)) hints to the dominance of the free electron contribution. This is not particularly surprising as the excursions of free carriers accelerated by an external force exceed the maximum amplitude of the trajectories of bound particles. However it is also possible that bound carrier contribution is not visible in $\Delta \phi_{N L}(t)$ due to propagation effects in the medium.

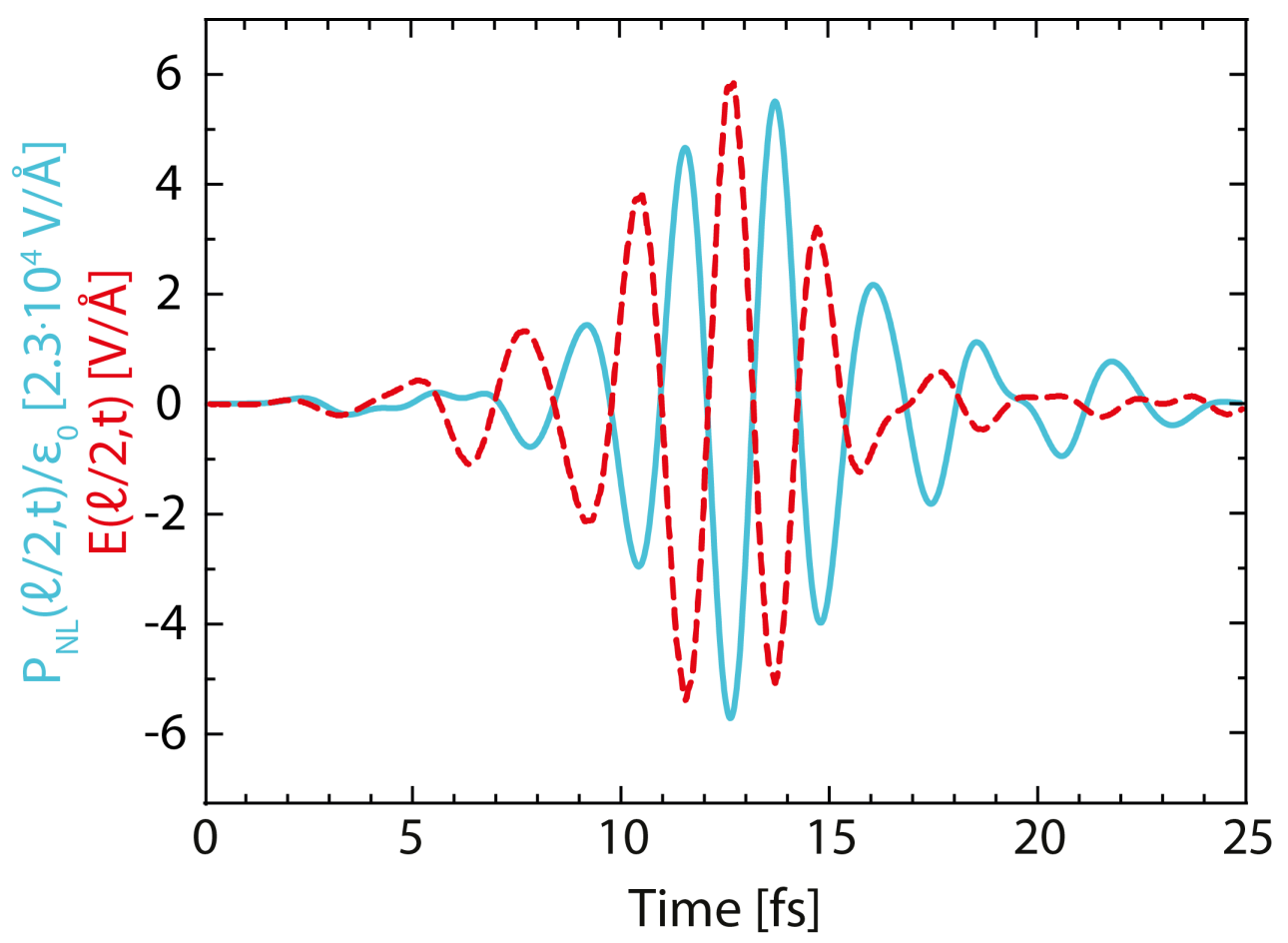

Figure 6.7: The strong field $E(\ell / 2, t)$ (red dashed line) at the midpoint of the medium is compared to the nonlinear polarization $P_{N L}(\ell / 2, t)$ (blue line) it induces.

Eqn. (6.10) facilitates the direct calculation of the time resolved nonlinear polarization induced by the electric field. As explained in section $6.1 P_{N L}(z, t)$ can be determined with 
highest precision at the midpoint of the medium. Therefore $E(\ell / 2, t)$ and $P_{N L}(\ell / 2, t)$ are compared after half the propagation distance (Fig.(6.7)). The induced nonlinear polarization wave reaches a maximum amplitude of $5.5 \cdot 10^{-5} \frac{\mathrm{C}}{\mathrm{m}^{2}}$ which corresponds to an electric field with a strength of $3.1 \cdot 10^{6} \frac{\mathrm{V}}{\mathrm{m}}$. This means that the driving field is more than 2000 times stronger than the nonlinear polarization it generates. Strikingly $E(\ell / 2, t)$ and $P_{N L}(\ell / 2, t)$ oscillate out of phase and are shifted by $\pi$ with respect to each other. This shift can be explained with Eqn. (2.49): Carriers which are released into the continuum at a peak of the electric field reach their maximum excursion close to the subsequent extremum of the driving wave. At this moment their velocity is zero as they are entirely decelerated. According to Eqn. (2.49) $P_{N L}(z, t)$ is directly proportional to the ensemble average position of the electron wavepacket. This means that $P_{N L}(z, t)$ and $E(z, t)$ are shifted by $\pi$ if the free electron contribution dominates the emitted nonlinear polarization wave.

As described above Eqn.(2.43) relates the measured change in refractive index to the generated free electron density $n_{e}(t)$. Its temporal evolution is depicted in Fig. $6.8 \mathrm{~A}$, light blue line) and is in a first order approximation considered to increase linearly with time (dark blue fit). Together with the extracted nonlinear polarization Eqn.(2.49) allows to determine the expectation value of the electron wavepacket $\langle x(t)\rangle$ during the strong field interaction. Fig. (6.8 B) shows that $\langle x(t)\rangle$ oscillates at the laser frequency with a maximum amplitude of $2.3 \mathrm{~nm}$. Even though the temporal evolution of $n_{e}(t)$ is approximated with a linear increase, the evaluated $\langle x(t)\rangle$ is in good agreement with theoretical predictions of more sophisticated models (N. Karpowicz, appendix 9.6.

The confirmation of the predicted decrease of the medium's refractive index due to photoionization verifies the applicability of Attosecond Polarization Spectroscopy. It can be used to investigate the nonlinear interaction between optical few-cycle pulses and matter with attosecond resolution. The findings in Neon provide direct real time access to the ionization rate. The determined nonlinear polarization contains information about the individual contribution of bound and free electrons to the plasma dynamics. It hints to the dominance of free carriers oscillating on length scales of a few nanometers.

The APS measurement in gas is primarily performed to verify the applicability of the experimental technique. In a next step it is interesting to further extend the investigation. By variation of the strong field amplitude different nonlinear interaction regimes can be explored. At intensities below the ionization threshold Kerr dynamics are expected to increase the refractive index of the material. A saturation of the nonlinearity at high field strength is controversially discussed [60-63]. If the field amplitude is stepwise increased above the ionization threshold the dynamics of plasma formation can be investigated in more detail. The results could be used to qualitatively and quantitatively review the different theoretical models describing the ionization process [56, 64, 65] and the plasma dynamics [39]. 


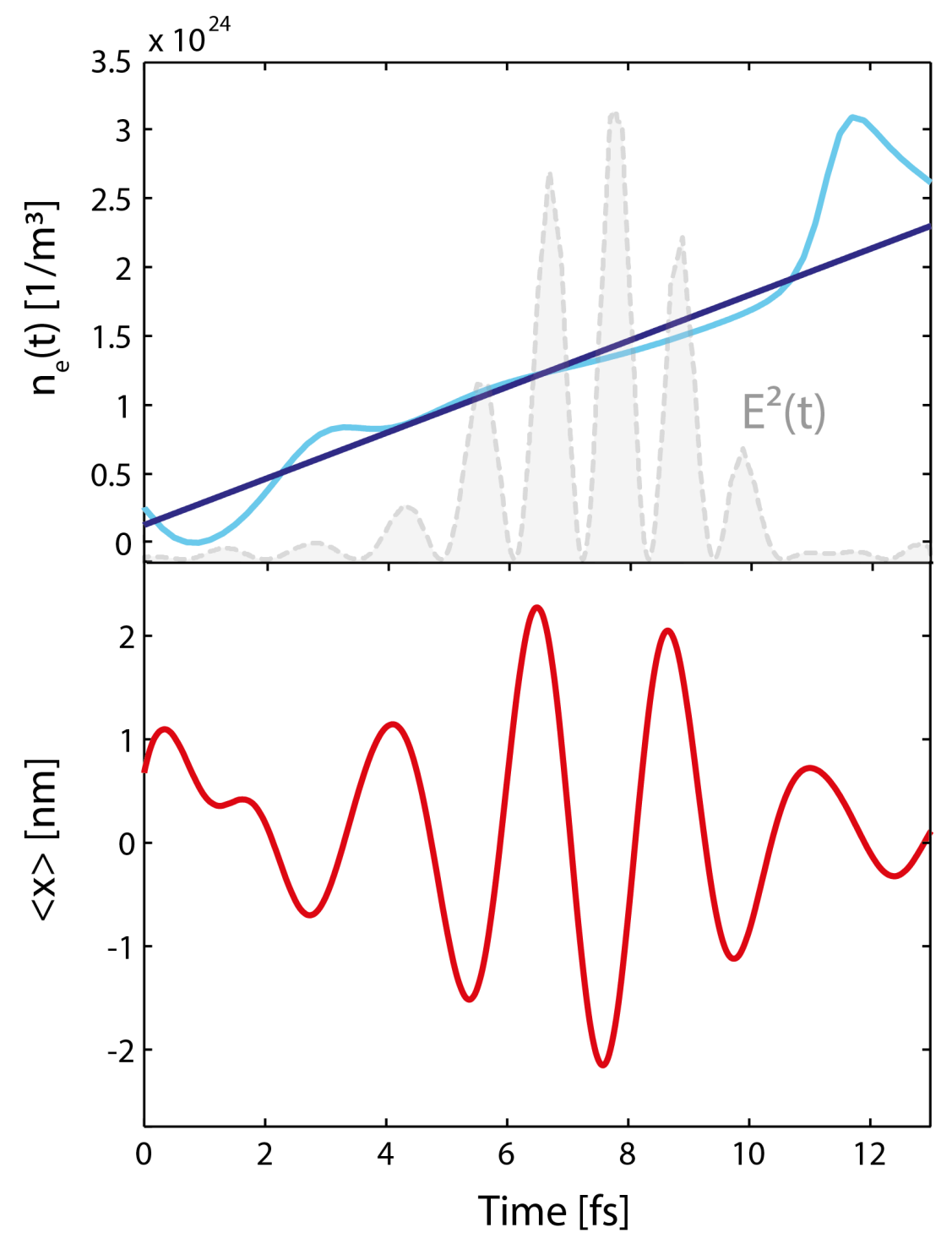

Figure 6.8: Panel A: The measured phase shift is used to calculate the time dependent plasma density (light blue line). In a first order approximation it is considered to increase linearly (dark blue line) throughout the pulse. The square of the electric field is additionally indicated (grey dashed line). Panel B: $n_{e}(t)$ and $P_{N L}$ are employed to extract the expectation value of the freed electron wave packet. 


\subsection{Nonlinear Interaction of few-cycle VIS/NIR Pulses with Solids}

The successful detection of the plasma contribution to the refractive index in gas proves the suitability of APS for the examination of ultrafast strong field dynamics. The evaluation of the nonlinear polarization with the derived theoretical concept provides physically reasonable and intuitively comprehensible results. In the following APS is employed to access the nonlinear response of dielectrics to few-cycle fields. The results presented in section 4 and section 5 reveal the characteristics of a nonlinear field effect reversible on femtosecond timescales. As APS allows to extract the nonlinear polarization it is expected to provide a more comprehensive picture of the interaction and extend the understanding of the underlying physical mechanisms.

APS is performed on two different dielectrics: at first the findings in fused silica are presented. Then they are compared to the results obtained in sapphire.

\subsubsection{The Nonlinear Refractive Index Change}

For the APS experiment in $\mathrm{SiO}_{2}$ the reference and strong field are transmitted through $10 \mu \mathrm{m}$ thick fused silica plates. The intensity of the weak field is adjusted to $I_{r e f}=(6.7 \pm 0.3)$. $10^{12} \frac{\mathrm{W}}{\mathrm{cm}^{2}}$ which corresponds to a field strength of $E_{\text {ref }}=(5.9 \pm 0.1) \cdot 10^{9} \frac{\mathrm{V}}{\mathrm{m}}$. The strong pulse possesses an intensity of $I=(1.3 \pm 0.1) \cdot 10^{14} \frac{\mathrm{W}}{\mathrm{cm}^{2}}$ which is equivalent to $E=(2.6 \pm 0.1) \cdot 10^{10} \frac{\mathrm{V}}{\mathrm{m}}$. The streaking spectrogram of both fields are displayed in appendix 9.4 (Fig.(9.11)).

As described in section 6.2 the reference measurement is always performed before and after the high intensity scan to verify the stability of the experimental setup. Fig.(6.9) displays $E_{r e f 1}(\ell, t)$ and $E_{r e f 2}(\ell, t)$ and demonstrates the excellent reproducibility of the recorded traces. The uncertainty of the relative timing is determined to $30 \mathrm{as}$. It is the average deviation in approximately 50 pairs of reference scans at the end of the pulse.

Fig. (6.10) compares the reference (dashed blue line) and the strong field (red line) after transmission through the medium and reveals an entirely different behavior than observed in the gas phase. With increasing electric field at the beginning of the pulse $E(\ell, t)$ is delayed with respect to $E_{r e f}(\ell, t)$. The accumulated temporal shift reaches a maximum value of $\Delta t_{\max }=290 \pm 40$ as which corresponds to a phase shift of $\Delta \phi_{N L \max }=0.7 \pm 0.1 \mathrm{rad}$. Towards the end of the pulse the delay decreases and finally vanishes entirely within the experimental uncertainty. 


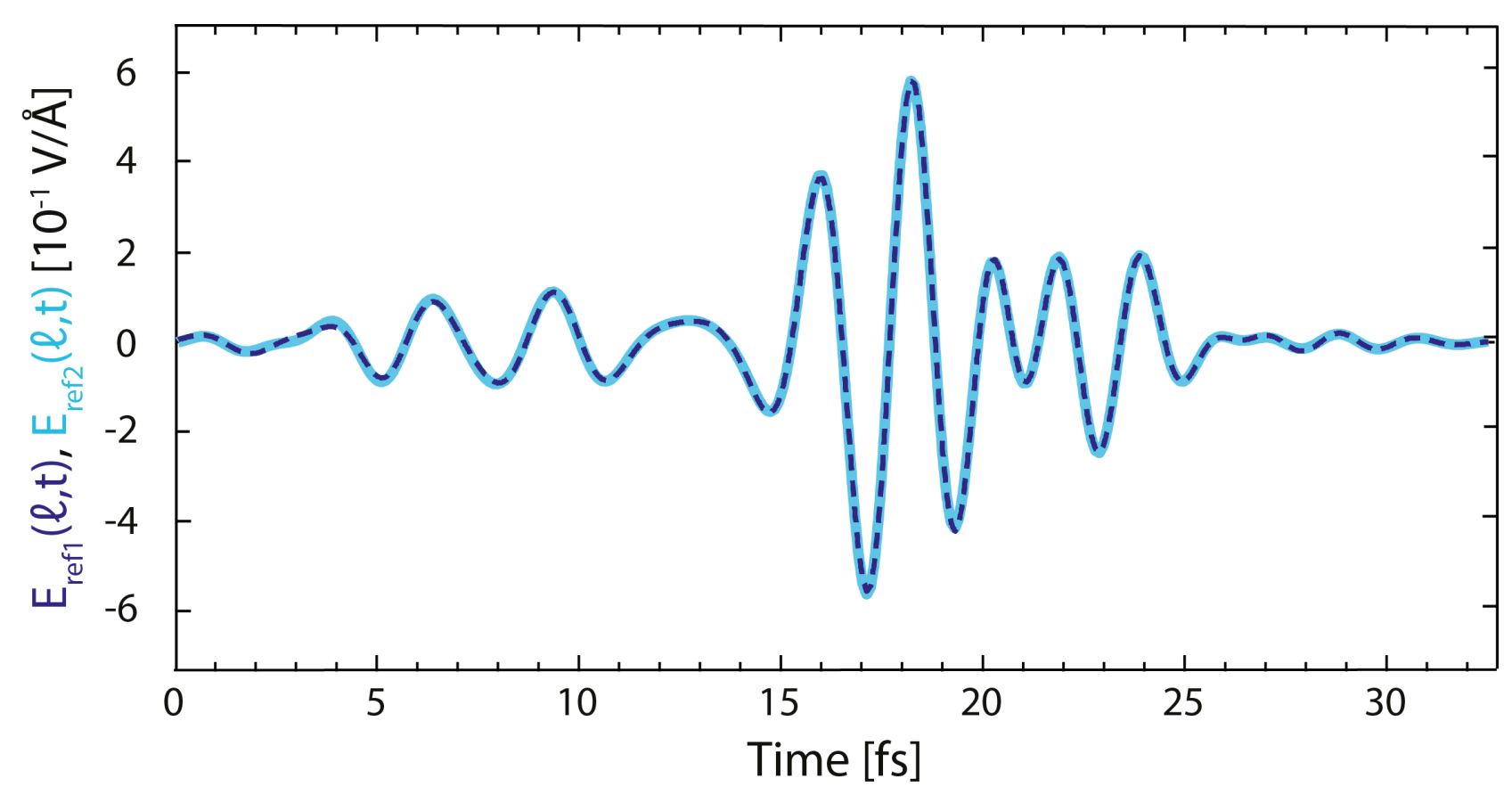

Figure 6.9: Comparison of the two reference fields $E_{r e f 1}(\ell, t)$ and $E_{r e f 2}(\ell, t)$. The excellent agreement proves the reproducibility of the experiment.

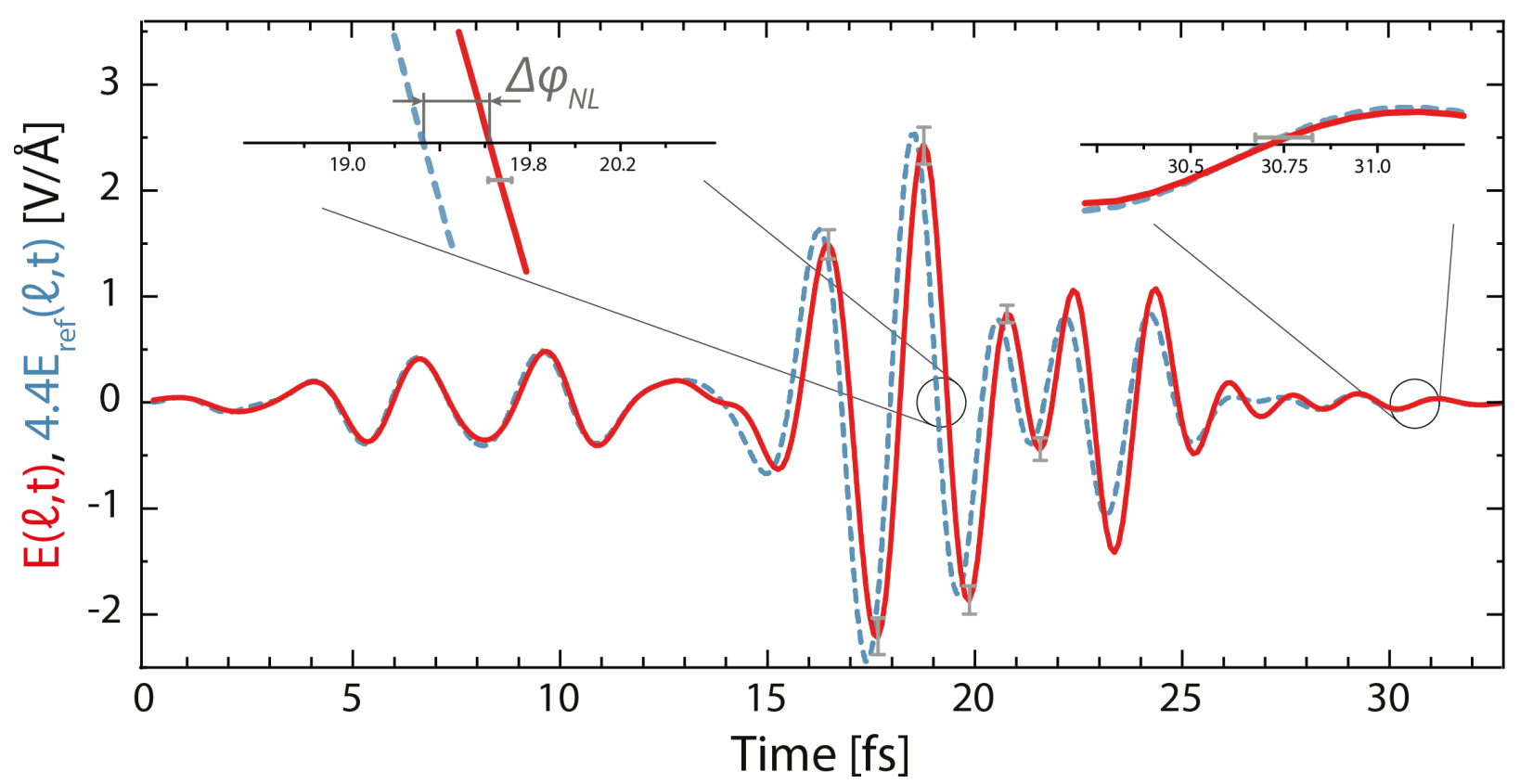

Figure 6.10: Comparison of the reference (blue dashed line) and strong electric field (red line) transmitted through $10 \mu \mathrm{m}$ fused silica at an intensity of $E_{r e f}=(5.9 \pm 0.1) \cdot 10^{9} \frac{\mathrm{V}}{\mathrm{m}}\left(\beta^{-1}=4.4\right)$ and $E=(2.6 \pm 0.1) \cdot 10^{10} \frac{\mathrm{V}}{\mathrm{m}}$. With increasing field strength $E(\ell, t)$ is shifted back with respect to $E_{r e f}$ (left inset). The phase shift recovers at the end of the pulse (right inset). 
The maximum phase shift $\Delta \phi_{N L \max }=0.7 \pm 0.1 \mathrm{rad}$ can be related to a relative change of the refractive index of $\delta n / n=0.6 \pm 0.1 \%$. According to the optical Kerr effect (Eqn.(2.22)) the optical material density increases proportional to the intensity envelope of the driving field. The Kerr prediction $\Delta \phi_{N L}(t)=k n_{2} I(t)$ with the nonlinear coefficient $n_{2}=$ $(1.1 \pm 0.3) \cdot 10^{-16} \frac{\mathrm{cm}^{2}}{W}$ determined in the Z-scan (section 3$)$ is displayed as the red dashed line in Fig. 6.11). The blue line depicts the experimentally determined temporal evolution of $\Delta \phi_{N L}$. It is obvious that neither the time profile nor the absolute amplitude of the two curves coincide. The Kerr prediction directly follows the intensity envelope of the driving field, whereas the measured temporal phase shift exhibits a pedestal-like structure around the main pulse, with sharp edges near the nodes of the intensity envelope. The discontinuity of $\Delta \phi_{N L}\left(t=21-22 f_{s}\right)$ is due to the local minimum of the pulse intensity in this range [46]. It is a numerical artifact and has no physical meaning.

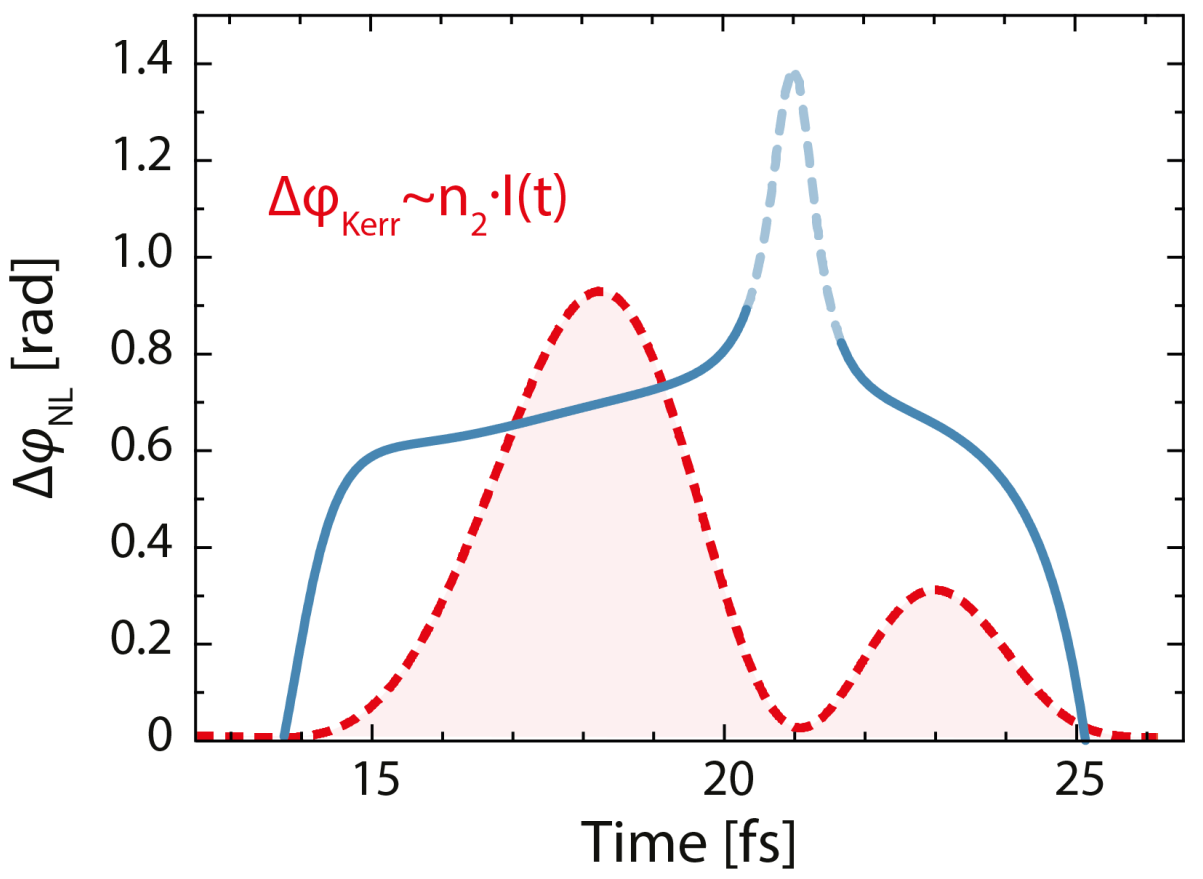

Figure 6.11: The difference $\Delta \phi_{N L}(t)$ between the phase of $E(\ell, t)$ and $E_{r e f}(\ell, t)$ (blue line) is compared to the prediction of the Kerr nonlinearity (dashed red line) which is proportional to the intensity envelope of the driving field. The Kerr response is calculated with $n_{2}=$ $(1.1 \pm 0.3) \cdot 10^{-16} \frac{\mathrm{cm}^{2}}{W}$ determined in the Z-scan measurement.

Simulations (appendix 9.8) confirm that the temporal profile of $\Delta \phi_{N L}(t)$ results from an interplay of nonlinearity and propagation effects for ultrashort broadband light fields. In the interaction of fused silica with femtosecond pulses the dispersion length (Eqn.(2.32) $k_{2}=446 \frac{f s^{2}}{c m}[33]$ ) exceeds the $10 \mu m$ material thickness by a factor of 20 . Therefore the influence of dispersion is expected to be negligible. Despite for the reported few-cycle pulses 
propagation significantly affects the temporal and spectral phase of the strong field. This is due to the fact that the information describing the nonlinear phase shift is more tightly confined in time than the pulse itself, but still influenced by dispersion in the subsequent material. This results in red-shifted light generated in the nonlinear material response outrunning the underlying pulse. These frequency components precede the high-intensity portion of the pulse and arrive at times where the fundamental field was zero. Hence the phase difference is very sensitive to changes at these points. In a similar way the blue-shifted components modify the phase shift behind the pulse.

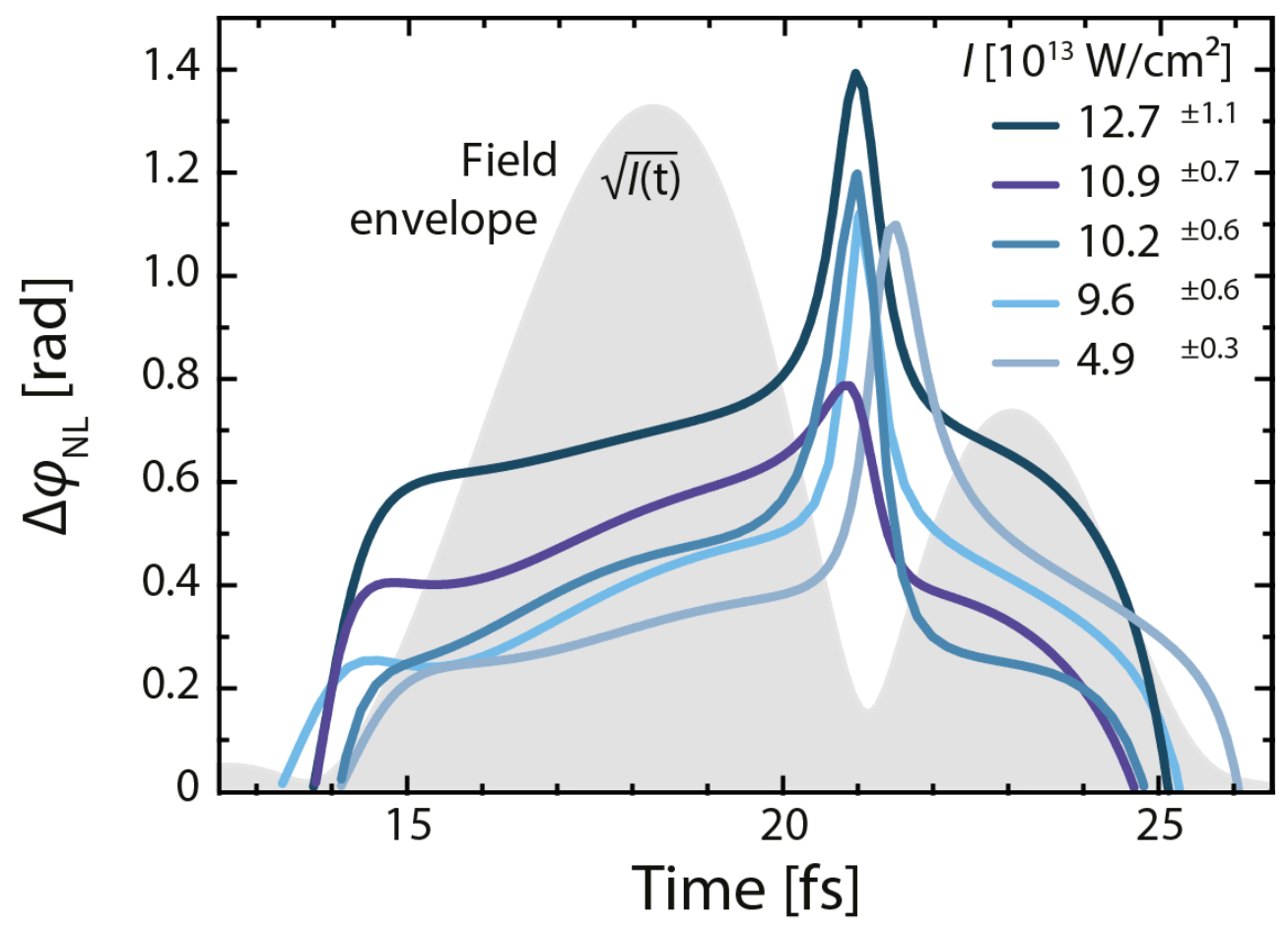

Figure 6.12: The phase difference $\Delta \phi_{N L}(t)$ between $E(\ell, t)$ and $E_{r e f}(\ell, t)$ for different peak intensities of $E(\ell, t)$ (increasing from light blue to dark blue). The field envelope is marked in gray.

The behavior of the Kerr nonlinearity at field strength close to optical breakdown is controversially discussed. It is not clear whether its amplitude saturates or even decreases close to the damage threshold of the material [60], [66]. To investigate this issue in the interaction of dielectrics with the reported few femtosecond pulses the peak intensity of $E(\ell, t)$ is continuously increased. The intensity scaling of $\Delta \phi_{N L}(t)$ is displayed in (Fig.(6.12)). It is striking that the overall temporal profile of $\Delta \phi_{N L}(t)$ seems to be independent of the maximum field amplitude the material is exposed to. However the phase shift attained at the peak of the field envelope $\Delta \phi_{N L \text { peak }}=\Delta \phi_{N L}\left(t=18-19 f_{s}\right)$ increases without any signs of saturation. As depicted in Fig.(6.13) the increase can be described as a linear rise with intensity. 


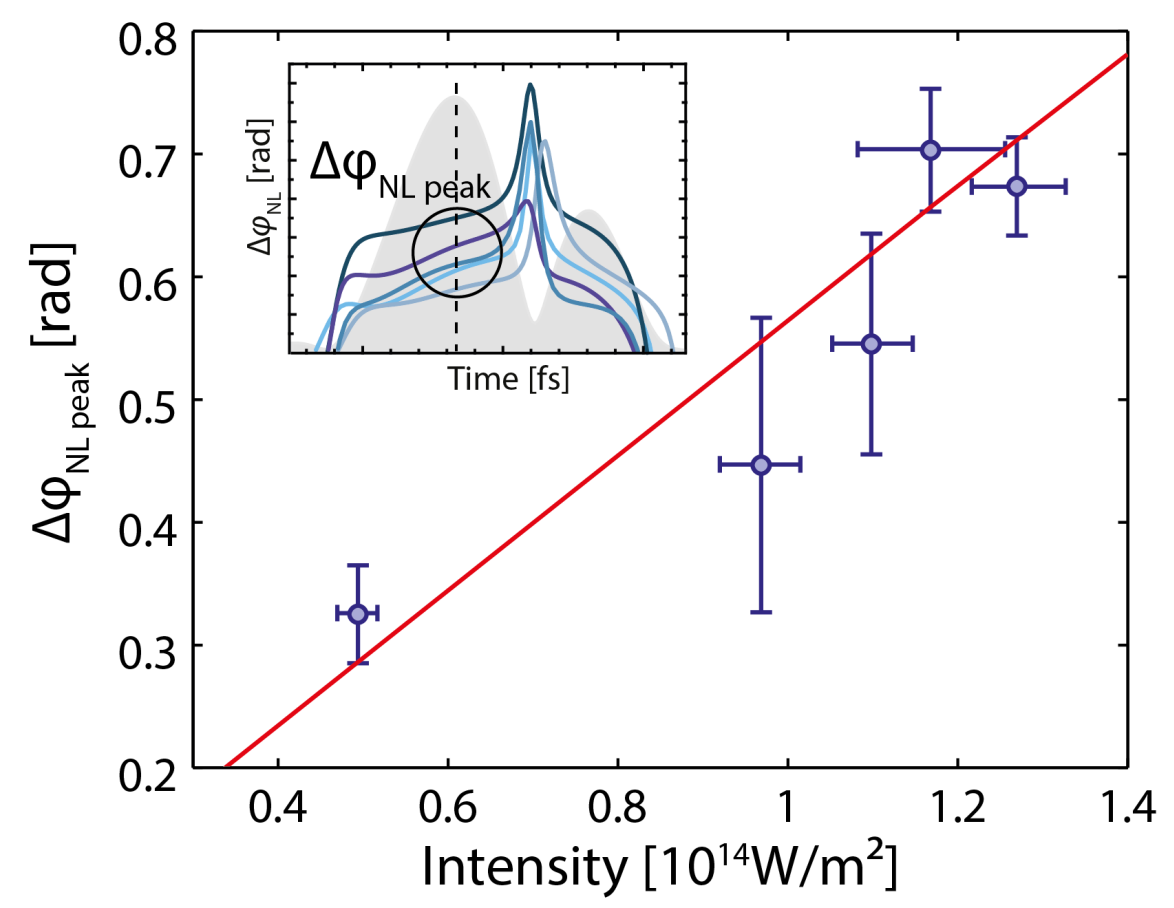

Figure 6.13: The induced nonlinear phase shift (blue circles) is evaluated at the peak of the field envelope (marked in the inset) for different intensities of the driving laser pulse. Within the experimental uncertainty $\Delta \phi_{N L \text { peak }}$ increases linearly with intensity (red line).

$$
\Delta \phi_{N L \text { peak }}\left(I_{\text {peak }}\right)=m \cdot I_{\text {peak }} \quad m=5.1 \cdot 10^{-19} \frac{\mathrm{rad}}{\mathrm{W}}
$$

Analogous to the Kerr effect a nonlinear coefficient $n_{2}$

$$
\begin{gathered}
m=k \ell n_{2} \\
n_{2}=6 \cdot 10^{-17} \frac{\mathrm{cm}^{2}}{\mathrm{~W}}
\end{gathered}
$$

can be extracted. This value is a factor 2 smaller than the result obtained in the time integrated Z-scan measurement (section 3) where the nonlinear phase shift is assumed to follow the intensity envelope of the field.

Careful comparison of the amplitude of $E(\ell, t)$ and $E_{r e f}(\ell, t)$ in Fig.(6.10) yields a decrease of the field strength of $E(\ell, t)$ at the beginning of the pulse $\left(16 f_{s}<t<19 f s\right)$ and an increase in the trailing part $(20 f s<t<25 f s)$. This behavior is even more pronounced in the modification of the field envelope depicted in Fig.(6.14). For the strong field $9 \pm 0.3 \%$ of the energy is redistributed from the main part of the pulse $\left(14 f_{s}<t<19 f s\right)$ to its tail ( $20 \mathrm{fs}<t<25 \mathrm{fs}$ ) in the interaction. The peak of the envelope is delayed by $\Delta \tau_{\text {peak }}=0.46 \pm 0.1 f$ s. This shift is predicted by the Kerr nonlinearity: inside the material 


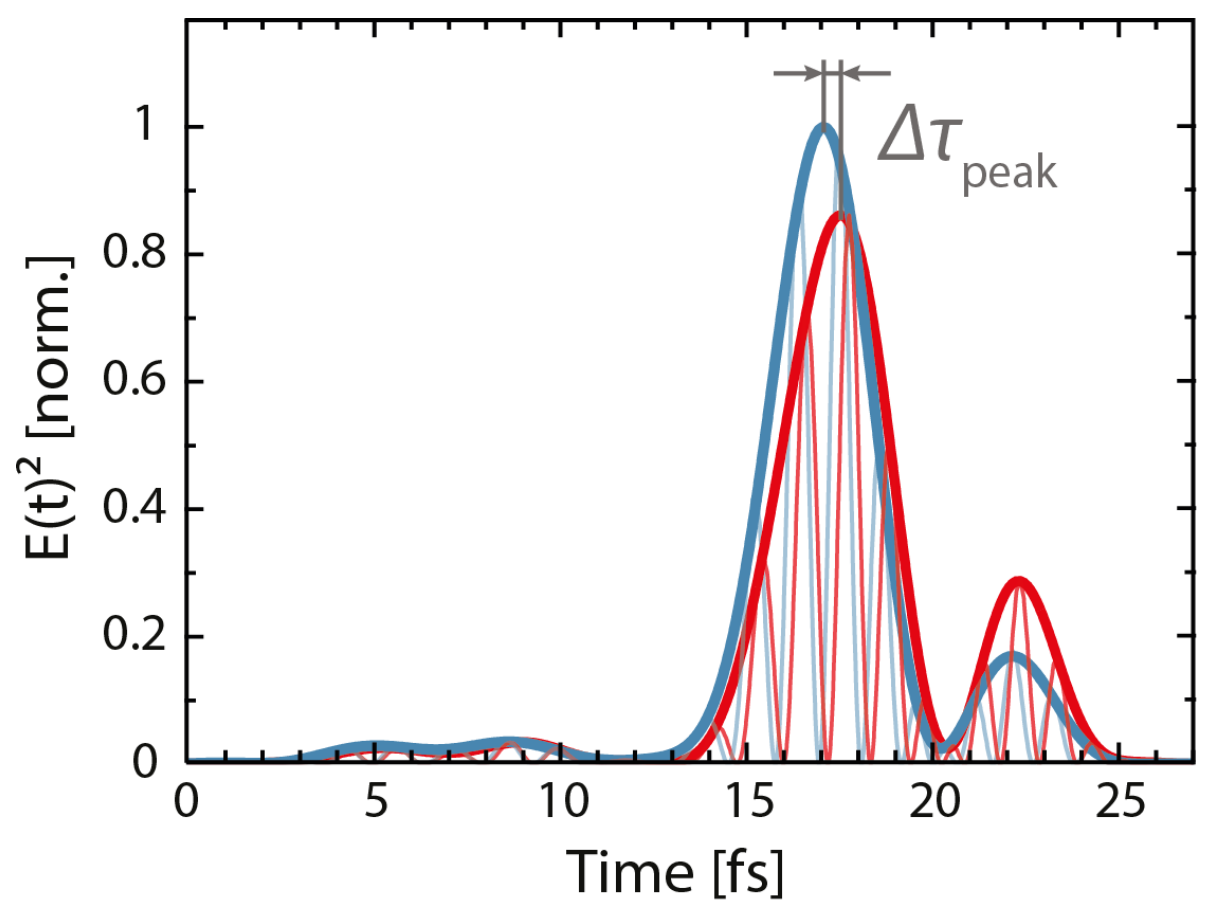

Figure 6.14: Comparison of the squared field envelope of $E(\ell, t)$ (red line) and $E_{r e f}(\ell, t)$ (blue line). The peak of $E(\ell, t)^{2}$ is delayed with respect to $E_{r e f}(\ell, t)$. Additionally a substantial amount of energy is redistributed from the main part of the pulse towards its tail.

the group velocity of the pulse becomes intensity dependent and decelerates the central part more strongly than the pulse flanks. This leads to a steepening of the trailing edge and a shift of the first momentum of the envelope (section 2.3). The delay $\Delta \tau_{\text {peak }}$ can be employed to calculate the nonlinear group velocity index $n_{2}^{g}=(1.2 \pm 0.2) \cdot 10^{-16 \frac{\mathrm{cm}^{2}}{W}}$ (Eqn. (2.31)).

$$
\begin{gathered}
\Delta n_{\text {eff }}^{g}=\frac{c \Delta \tau_{\text {peak }}}{\ell} \\
\Delta n_{\text {eff }}^{g}=n_{2}^{g} I
\end{gathered}
$$

Far away from resonance the nonlinear Kerr coefficient $n_{2}$ and the group velocity index are expected to coincide [33]. However $n_{2}^{g}$ exceeds the value extracted for $n_{2}$ from the intensity scaling of $\Delta \phi_{\text {NLpeak }}$ (Fig. (6.12) ) by a factor of 2. A possible explanation for this discrepancy can be given by assuming that the nonlinear response of the medium is influenced by an electronic and a Raman contribution. As the electron dynamics evolve on shorter timescales than the lattice excitations, the electronic contribution dominates the nonlinearity in the pulse center where the field strength is highest. At the trailing edge of the field the Raman processes which oscillate at lower frequencies become visible. For intra pulse Raman scattering blue photons at the pulse front excite electrons into virtual levels with finite lifetime. Upon their decay optical phonons are excited and a red shifted photon is emitted. Like this energy is transferred from the front to the tail of the pulse (section 2.5). The value for $n_{2}$ evaluated 
from the intensity scaling of $\Delta \phi_{\text {NLpeak }}$ at the field maximum is dominated by the nonlinear electronic processes. The delay of the intensity envelope $\Delta \tau_{\text {peak }}$ is influenced by both, the electronic and Raman dynamics. Hence the result for $n_{2}^{g}$ exceeds the $n_{2}$ extracted from $\Delta \phi_{\text {NLpeak }}$.

The value of the group velocity index is in good agreement with the nonlinear Kerr coefficient extracted from the Z-scan (section 3). The time integrated measurement cannot distinguish between the electronic and lattice contribution to the nonlinearity. Therefore its result coincides with $n_{2}^{g}$.

Remarkably the induced phase shift is reversible up to field strength close to optical breakdown within the experimental uncertainty (Fig.(6.10)). This observation hints to a negligible amount of real carriers excited into the conduction band by the external field. $\mathrm{V}$. Temnov et al. determined the lifetime of real conduction band population excited via multiphoton absorption in the interaction of fused silica with $50 \mathrm{fs}$ pulses to approximately $200 f s$ [67]. This shows that real carriers generated by the few femtosecond pulse would survive the interaction period and decay after the pulse already vanished. As verified for the plasma dynamics in Neon free carriers decrease the optical density of the medium. If the ultrashort light field excites a considerable fraction of electrons into the conduction band, this population generates a negative phase shift which does not recover at the end of the pulse. This is not detectable in the experiment.

To estimate the maximum amount of excited carriers which is not detectable within the experimental uncertainty, the residual nonlinear phase shift after the pulse is determined. It is evaluated as the average of 30 independent measurement sets recorded at $I \approx 1 \cdot 10^{14} \frac{\mathrm{W}}{\mathrm{cm}^{2}}$ to $\Delta \phi_{N L}(t \approx 30 \mathrm{fs})=0.16 \pm 0.09 \mathrm{rad}$. This corresponds to a refractive index change of $\Delta n<(2 \pm 1.1) \cdot 10^{-3}$. According to Eqn. 2.47) this $\Delta n$ is induced by a free electron density of $n_{e}<(1.1 \pm 0.5) \cdot 10^{19} \frac{1}{\mathrm{~cm}^{3}}$. The average density of valence band electrons in quartz is $1.4 \cdot 10^{23} \frac{1}{\mathrm{~cm}^{3}}$ (atomic density $2.7 \cdot 10^{22} \frac{1}{\mathrm{~cm}^{3}}$, oxygen 6 valence electrons, silicon 4 ). This means that less than $0.008 \pm 0.005 \%$ of the valence electrons remain excited in the conduction band after the pulse. Therefore the interaction can be claimed as entirely reversible on femtosecond timescales.

\subsubsection{Comparison of the Nonlinear Refractive Index of Different Dielectrics}

The time resolved investigation of the strong field dynamics in fused silica reveals that the Kerr effect alone cannot describe the temporal evolution of the nonlinearity for few-cycle pulses. The optical material density is not modified proportional to the intensity envelope of the driving field but exhibits a more sophisticated temporal structure. It is caused by the interaction of nonlinearity and dispersion during the propagation of the broadband light field. There is no indication for saturation of the nonlinear effect up to field strength close to the damage threshold ((appendix 9.7)) of the material. Comparison of the nonlinear modification of the carrier wave and the field envelope hint to a contribution of the lattice to the light-matter interaction on few femtosecond timescales. 
It is interesting to investigate whether the observed behavior is unique in fused silica or universal for wide-gap solids. To examine this question the APS measurement is repeated in sapphire. Fused silica and sapphire possess a bandgap of similar size. Apart from this communality their energetic, crystal and chemical structure does not resemble each other.

In the experiment $25 \mu \mathrm{m}$ thin $\mathrm{Al}_{2} \mathrm{O}_{3}$ plates are exposed to few-cycle pulses with an intensity of $I=(8.1 \pm 0.5) \cdot 10^{13} \frac{\mathrm{W}}{\mathrm{cm}^{2}}\left[E=(1.9 \pm 0.1) \cdot 10^{10} \frac{\mathrm{V}}{\mathrm{m}}\right]$. To record the reference trace the intensity is reduced by a factor of 13.5 to $I_{r e f}=(0.6 \pm 0.02) \cdot 10^{13} \frac{\mathrm{W}}{\mathrm{cm}^{2}}\left[E_{\text {ref }}=\right.$ $\left.(0.5 \pm 0.08) \cdot 10^{10} \frac{\mathrm{V}}{\mathrm{m}}\left(\beta^{-1}=3.7\right)\right]$. The electric fields extracted from the recorded streaking spectrogram are depicted in Fig.(6.15).

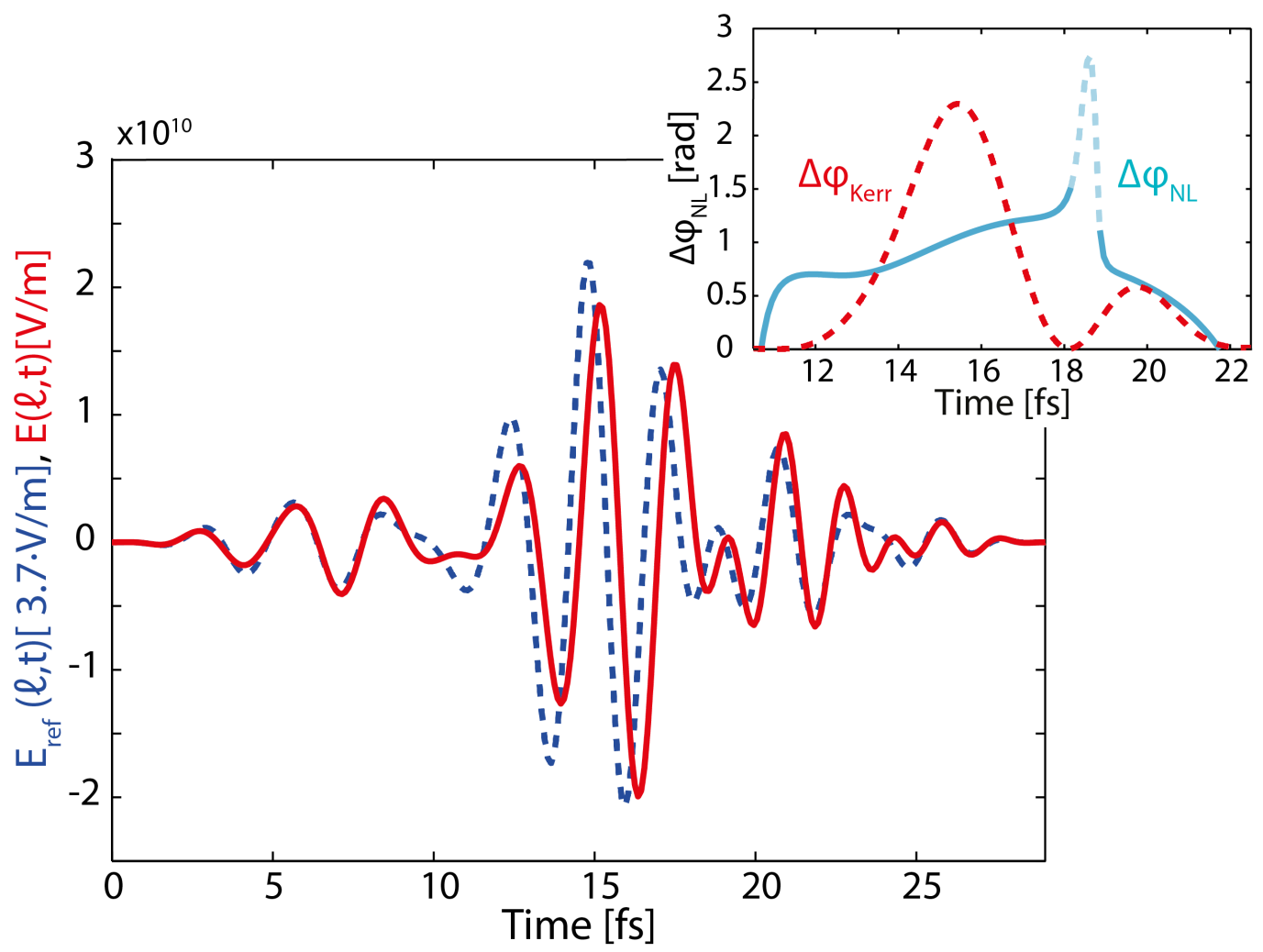

Figure 6.15: Comparison of the electric field $E_{r e f}(\ell, t)=(0.5 \pm 0.08) \cdot 10^{10} \frac{\mathrm{V}}{\mathrm{m}}\left(\beta^{-1}=3.7\right)$ (blue dashed line) and $E(\ell, t)=(1.9 \pm 0.1) \cdot 10^{10} \frac{\mathrm{V}}{\mathrm{m}}$ (red line) transmitted through $25 \mu \mathrm{m}$ sapphire. The evolution of the nonlinear phase $\Delta \phi_{N L}$ is displayed in the inset (light blue line) together with the prediction of the Kerr effect $\Delta \phi_{\text {Kerr }}$ (red dashed line).

Similar to the behavior observed in fused silica the strong field is increasingly delayed with respect to $E_{r e f}(\ell, t)$ in the central part of the pulse. When the field strength decreases the phase shift $\Delta \phi_{N L}$ is gradually reduced and recovers at the end of the pulse. $\Delta \phi_{N L}$ reaches a maximum value of $\Delta \phi_{N L \max }=1.3 \pm 0.1 \mathrm{rad}$ which corresponds to a relative change in refractive index of $\Delta n / n=0.4 \pm 0.03 \%$. For comparison, the optical density of fused silica is modified by $0.35 \%$ at an peak intensity of $8 \cdot 10^{13} \frac{\mathrm{W}}{\mathrm{cm}^{2}}$.

The temporal profile of $\Delta \phi_{N L}$ is displayed in the inset in Fig.6.15 light blue line). The 
Kerr prediction (red dashed line) is estimated with the nonlinear Kerr coefficient of $n_{2}=$ $(1.3 \pm 0.4) \cdot 10^{-16} \frac{\mathrm{cm}^{2}}{W}$ extracted with the Z-scan technique. Obviously $\Delta \phi_{N L}$ is not proportional to the intensity envelope of the field but exhibits a pedestal-like shape similar to the profile observed in $\mathrm{SiO}_{2}$. As analyzed in the previous chapter this behavior results from the interplay of nonlinearity and dispersion for ultrashort pulses (section 6.4.3).

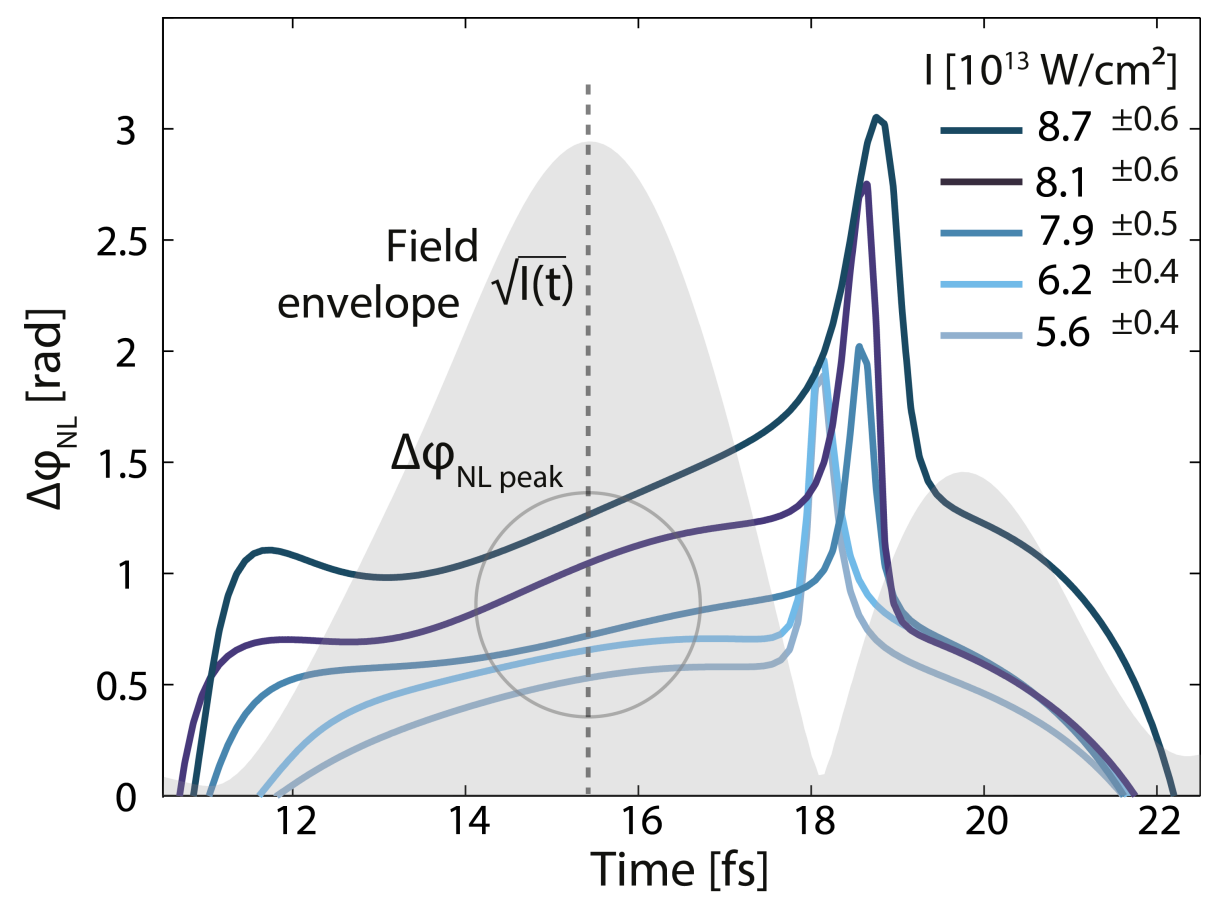

Figure 6.16: The dependence of the nonlinear phase shift $\Delta \phi_{N L}(t)$ between $E_{r e f}(\ell, t)$ and $E(\ell, t)$ on the peak intensity of the strong field. The intensity is increases from light to dark blue. The field envelope is displayed in gray.

The variation of $\Delta \phi_{N L}(t)$ with the maximum field strength of the strong pulse is depicted in Fig. 6.16), where the peak intensity is increased stepwise from $I=(5.6 \pm 0.4) \cdot 10^{13} \frac{\mathrm{W}}{\mathrm{cm}^{2}}$ (light blue line)) to $I=(8.7 \pm 0.6) \cdot 10^{13} \frac{\mathrm{W}}{\mathrm{cm}^{2}}$ (dark blue line)). Similar to the observation in fused silica (Fig. (6.12) ) the pedestal like shape of $\Delta \phi_{N L}(t)$ throughout the pulse seems to be independent of the peak intensity the material is exposed to. Again the value of $\Delta \phi_{N L}$ peak at the maximum of the field envelope (Fig.(6.16) ) rises linearly with intensity. No indication of saturation is visible. Analogue to the procedure performed for $\mathrm{SiO}_{2}$ (section 6.4.1) a nonlinear Kerr coefficient $n_{2}=0.65 \cdot 10^{-16} \frac{\mathrm{cm}^{2}}{W}$ (Eqn. $(6.21)$ ) can be extracted from the intensity scaling of $\Delta \phi_{N L}$ peak. This value is by a factor 2 smaller than the $n_{2}$ evaluated in the Z-scan (section 3.1).

The field envelope of $E_{r e f}(\ell, t)$ and $E(\ell, t)$ after the interaction with the material is depicted in Fig.6.17 A). The peak of $E(\ell, t)^{2}$ is delayed by $\Delta \tau_{\text {peak }}=770 \pm 210$ as with respect to $E_{r e f}(\ell, t)^{2}$. This shift corresponds to a nonlinear group velocity index of $n_{2}^{(g)}=$ $(1.5 \pm 0.4) \cdot 10^{-16} \frac{\mathrm{cm}^{2}}{W}$ (Eqn. 2.31)). In fused silica and sapphire $n_{2}^{(g)}$ coincides well with the 

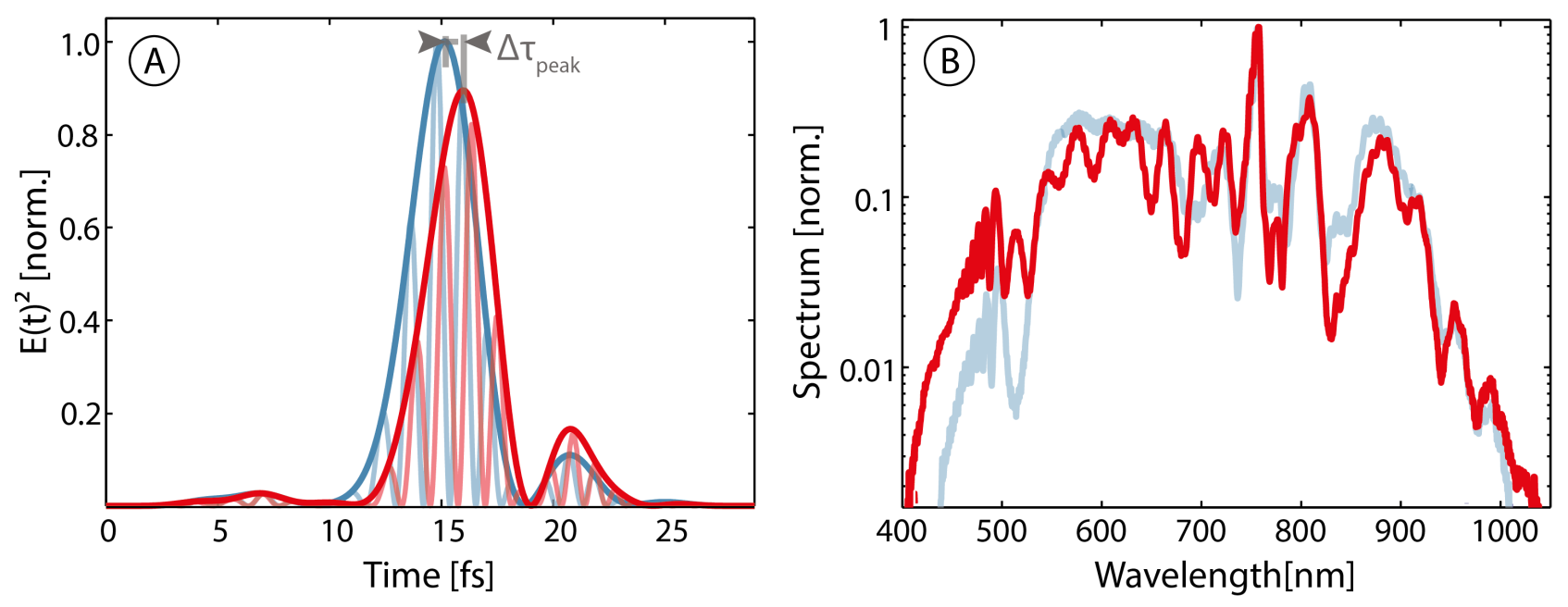

Figure 6.17: Comparison of the field envelope (Panel A) and spectra (Panel B) of $E_{r e f}(\ell, t)$ (blue line) and $E(\ell, t)$ (red line) after the transmission through $25 \mu m$ sapphire.

nonlinear Kerr coefficient measured in the $\mathrm{Z}$ scan. In both cases it is twice as big as the $n_{2}$ evaluated from $\Delta \phi_{N L}$ peak. As described in section 6.4.1 this discrepancy can be explained with the different timing of the electronic and lattice contribution to the nonlinearity.

Comparison of the integral over $I(\ell, t)$ and $I_{r e f}(\ell, t)$ reveals that the area below $I(\ell, t)$ contains $11 \%$ less energy than for the reference field. There are two possible explanations for this discrepancy. Either the energy is absorbed by the material. It is also possible that the pulse spectrum is modified in the nonlinear interaction and new frequency components are generated which are delayed and do not appear in the time window of the streaking measurement. To find out which explanation is correct the pulse energy of the weak and the strong field is additionally determined in a time integrated power measurement. Under consideration of the attenuation of the reference field the energy of both pulses is identical. The spectra of both fields are compared Fig. (6.17) B). It is apparent that new frequency components are generated in the nonlinear interaction at both sides of the fundamental spectrum. The entire frequency range of the strong field is modulated at a slowly oscillating beating frequency. This interference pattern corresponds to a double pulse structure in time. These findings prove that photons of the fundamental strong field are converted to new frequencies in the nonlinear interaction (section 2.4). Most probably due to propagation effects these generated spectral components are delayed to the driving pulse and do not appear in the time window of the streaking measurement.

The APS experiment with fused silica is performed on sample plates which are 2.5 times thinner than the sapphire targets. In these thin samples no modifications of the spectral composition of the light field are detectable after the nonlinear interaction.

The results obtained with APS in $\mathrm{SiO}_{2}$ and $\mathrm{Al}_{2} \mathrm{O}_{3}$ resemble each other very strongly. For both materials the temporal evolution of the induced nonlinear phase shift $\Delta \phi_{N L}$ is dominated by the interplay of nonlinearity and propagation effects. Up to field strength close to optical breakdown the amplitude of the nonlinear effect rises linearly with intensity. 
In both dielectrics there is evidence of a Raman contribution to the strong field effect on femtosecond timescales in addition to the triggered electronic nonlinearity. Fused silica and sapphire possess a completely different chemical and crystalline composition. The only communality they share is the classification as wide-gap solids. The strong similarity of the experimental results for the two different dielectric hints to an universal response mechanism of insulators to intense few-cycle pulses.

\subsubsection{The Nonlinear Polarization Response}

The nonlinear polarization completely characterizes the nonlinear response of matter to an intense electric field. It is the essential quantity to gain insight into the excited strong field dynamics and understand their physical origin. As explained in section 5 so far the nonlinear polarization induced by optical few-cycle pulses in dielectrics could only be extracted from theoretical simulations (Fig.(6.18)). Attosecond Polarization Spectroscopy provides access to its complete amplitude and phase information via the analytic relation described by Eqn.(6.10).

At first the nonlinear polarization induced in fused silica is evaluated for the data set depicted in Fig.(6.10). The $10 \mu \mathrm{m}$ fused silica sample is exposed to a light pulse with a peak intensity of $I=(1.3 \pm 0.1) \cdot 10^{14} \frac{\mathrm{W}}{\mathrm{cm}^{2}}$ close to the optical breakdown of the material. As the reliability of the extracted nonlinear polarization is highest at half the propagation distance (section 6.1), $P_{N L}(\ell / 2, t)$ is compared to the strong field $E(\ell / 2, t)$ at the midpoint of the material (Eqn.(6.15)).

The results are displayed in Fig.(6.18). When the electric field strength increases at the beginning of the pulse $P_{N L}(\ell / 2, t)$ is delayed with respect to the strong field. The shift decreases towards the center of the pulse until the polarization wave overtakes $E(\ell / 2, t)$. This reversal is caused by the inversion of the energy transfer between field and material which will be analyzed in more detail in the following section. With rising field strength the electric wave performs an increasing amount of work to displace the electrons from their equilibrium position and transfers energy to the medium. As explained in the next chapter this energy flow is directly related to the delay of the polarization wave to the strong field. When the field strength decreases the electronic system partially returns to its unperturbed state and energy flows back to the medium. This reversal of the flow direction shifts $P_{N L}(\ell / 2, t)$ to earlier times.

In the regime where energy is transferred from the light field to the medium, the delay between the nonlinear polarization and the driving field can be considered as an upper limit for the response time of the electronic system. This is the time which an external field requires to distort the electronic distribution in a medium. In dielectrics excited far from resonance this nonlinear process is assumed to be very fast. The response time can be estimated by the orbital period of the electron circling around the nucleus [33].

$$
\tau \approx \frac{2 \pi a_{B}}{v_{e}}
$$




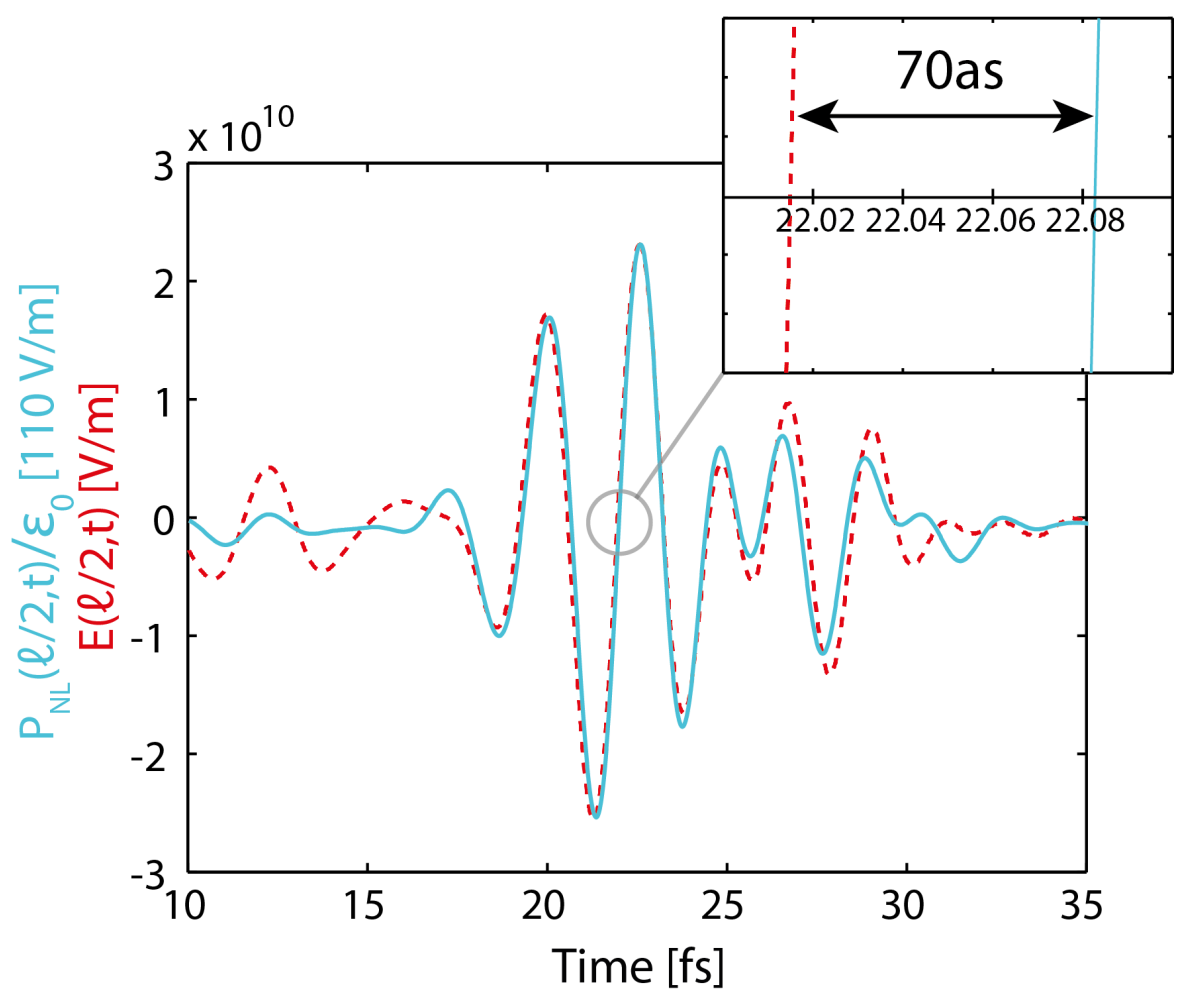

Figure 6.18: The nonlinear polarization (light blue line) which the intense laser pulse induces in fused silica is extracted with Eqn.(6.10). It is compared to the strong field (red dashed line) after half the propagation distance through the medium. The delay between the two waves in the zero crossing of the central flank determines an upper boundary for the electronic response time.

With $a_{B}=5.3 \cdot 10^{-11} \mathrm{~m}$ and $v_{e} \approx c / 137, \tau$ is assumed to be of the order of a few 100 as. The results of APS can be used to scrutinize this estimation. The shift between $P_{N L}(\ell / 2, t)$ and $E(\ell / 2, t)$ can be evaluated with highest precision in the zero crossing of the central flank were the slope of the two fields is steepest. The comparison yields a delay of $70 \pm 30$ as (Fig.(5.4) inset). Therefore an upper limit of $\tau_{\text {res elec }}<100$ as can be set for the electronic response time of fused silica to the reported few-cycle pulses at a field strength close to optical breakdown (here $E=2.6 \pm 0.1 \mathrm{~V} / \AA$ ).

The response time is evaluated for different peak amplitudes of the strong field. Fig.(6.19) displays the results of 7 individual traces recorded at intensities between $0.7-1.4 \cdot 10^{14} \frac{\mathrm{W}}{\mathrm{cm}^{2}}$. It shows the rising tendency of $\tau_{\text {res elec }}$ with increasing intensity. For $I<1 \cdot 10^{14} \frac{\mathrm{W}}{\mathrm{cm}^{2}}$ the amplitude of the induced nonlinear polarization and its delay to the driving field becomes very small. This reduces the signal to noise ratio and increases the error bar of $\tau_{\text {res elec }}$ compared to higher field strength. Hence the value of the evaluated response time scatters considerably around $20 \pm 25$ as in this range. However the trend of a slower response with rising field strength is visible. As explained in the next section the delay of the nonlinear polarization to the strong field is directly related to the energy transferred from the field to 


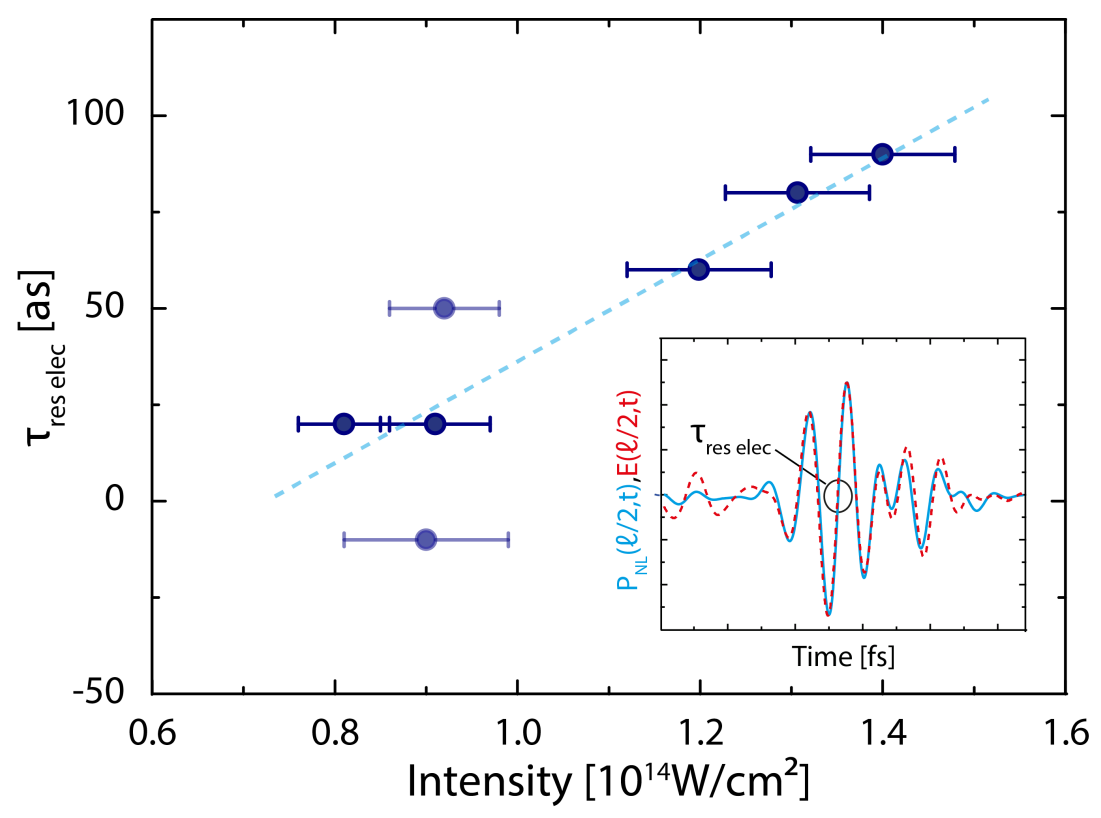

Figure 6.19: The dependency of the electronic response time on the intensity of the external field. The response time is evaluated as the delay between the nonlinear polarization wave and the strong field in the zero crossing of the central flank (inset).

the material. As this delay defines $\tau_{\text {res elec }}$, the increase in absorption with rising field strength, decelerates the electronic response.

\section{Comparison of the Nonlinear Polarization in Different Dielectrics}

It is interesting to investigate whether the response time of different dielectrics is of the same order of magnitude and exhibits a similar intensity dependence as in fused silica. Therefore the nonlinear polarization induced in the $25 \mu \mathrm{m}$ sapphire samples by a field with a peak intensity of $I=(8.1 \pm 0.5) \cdot 10^{13} \frac{\mathrm{W}}{\mathrm{cm}^{2}}$ (Fig.(6.15)) is evaluated with Eqn.(6.10). It is displayed in Fig. 6.20 A, blue line). $P_{N L}(\ell / 2, t)$ exhibits the same temporal characteristics as the nonlinear material response of fused silica: at the beginning of the pulse the external wave transfers energy to the material. This energy flow delays the nonlinear polarization wave to the strong field. When the field amplitude decreases the electrons partially return energy to the field as they come back to their initial unperturbed states. Then the nonlinear polarization wave precedes the strong field. For a peak intensity of $I=(8.1 \pm 0.5) \cdot 10^{13} \frac{\mathrm{W}}{\mathrm{cm}} \mathrm{m}^{2}$ an electronic response time of $\tau_{\text {res elec }}=40 \pm 30$ as is evaluated by comparing $P_{N L}(\ell / 2, t)$ to $E(\ell / 2, t)$ in the zero crossing of the central flank. Therefore an upper limit of $\tau_{\text {res elec }}<70 a$ s is claimed.

As derived in Eqn.(6.8) and experimentally verified in section 6.4.6 the nonlinear modification of the strong field scales linearly with the propagation distance. The sapphire samples are 2.5 times thicker than the fused silica targets. As the refractive index of the two materials possess similar values the nonlinear phase shift accumulated in $\mathrm{Al}_{2} \mathrm{O}_{3}$ is approximately 2.5 times bigger than in $\mathrm{SiO}_{2}$. This improves the signal to noise ratio in the evaluation of $P_{N L}(t)$ 

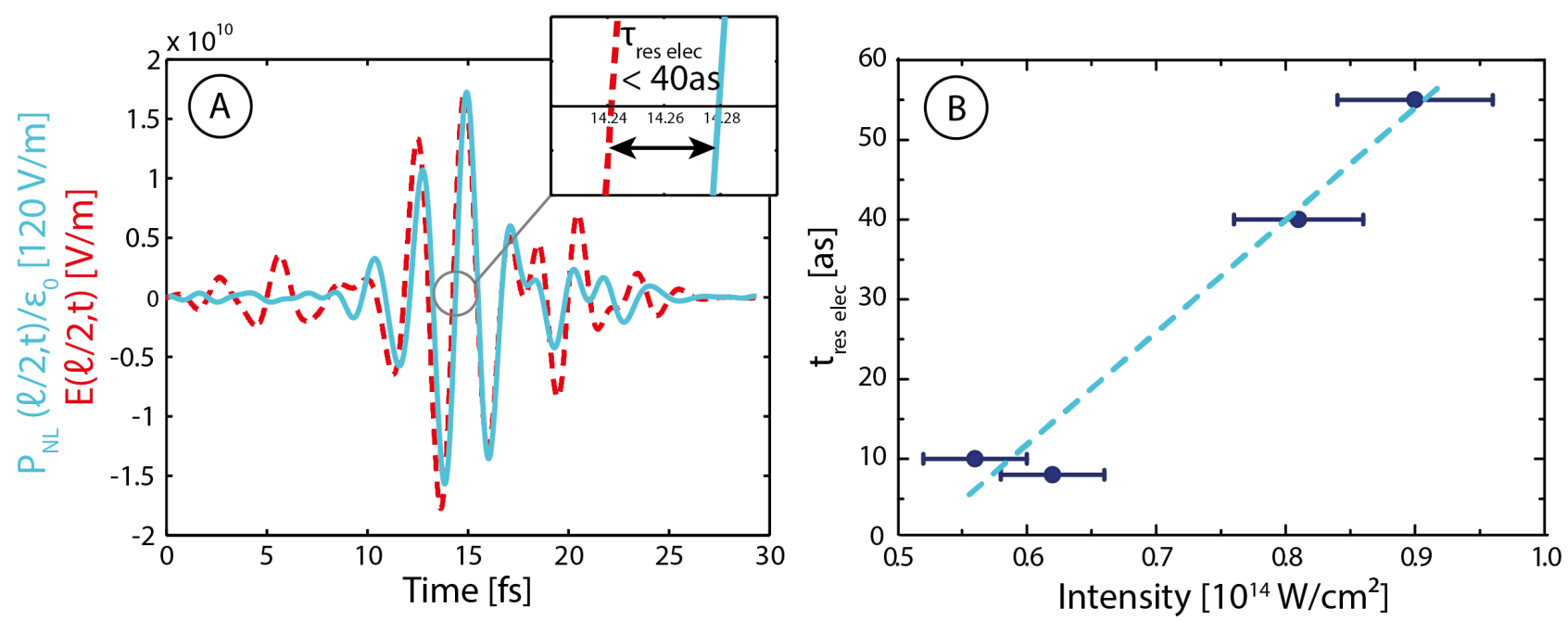

Figure 6.20: Panel A: The nonlinear polarization (blue line) is compared to the strong field (red dashed line) at the middle of the sapphire sample. Comparison of the delay between $P_{N L}(\ell / 2, t)$ and $E(\ell / 2, t)$ in the zero crossing of the central flank allows to extract the electronic response time of the system (inset). Panel B: $\tau_{\text {res elec }}$ clearly increases with the peak intensity of the driving field.

especially for fields with low peak intensities. On the other hand the validity of Eqn.(6.7) decreases with growing interaction length. It assumes all nonlinear dipoles to oscillate in phase and be driven by the same nearly-undistorted waveform. Fig.(6.17B) shows that the spectral composition of the driving field is slightly altered in the interaction. However the modifications are still small enough to have negligibly affect on the pulse shape. It is possible to reliably evaluate the nonlinear polarization and the response time of sapphire down to intensities of $6 \cdot 10^{13} \frac{\mathrm{W}}{\mathrm{cm}^{2}}$. Fig.(6.20 B) shows a clear increase of $\tau_{\text {res elec }}$ with rising intensity. This is the same trend as observed for fused silica. This finding hints to an universal intensity dependent delay of the nonlinear response in wide-gap materials. For field strength close to optical breakdown an upper boundary of $\tau_{\text {res elec }}<100$ as is claimed.

\subsubsection{Energy Transfer Dynamics}

The knowledge of the nonlinear polarization does not only allow to extract the electronic response time of the system but provides access to the energy transfer dynamics during the light-matter interaction. It is possible to investigate when and to which amount the material gains or looses energy.

The time resolved energy density $W(t)$ transferred between the electric field and the material is accessible via Eqn.(6.16). It is displayed in Fig.(6.21) for fused silica and an intensity of the driving field of $I=(1.3 \pm 0.1) \cdot 10^{14} \frac{\mathrm{W}}{\mathrm{cm}^{2}}$. With rising field strength the external wave distorts the electron cloud in the material and moves the carriers further and further away from their equilibrium position. It performs work to modify the energetic structure of 


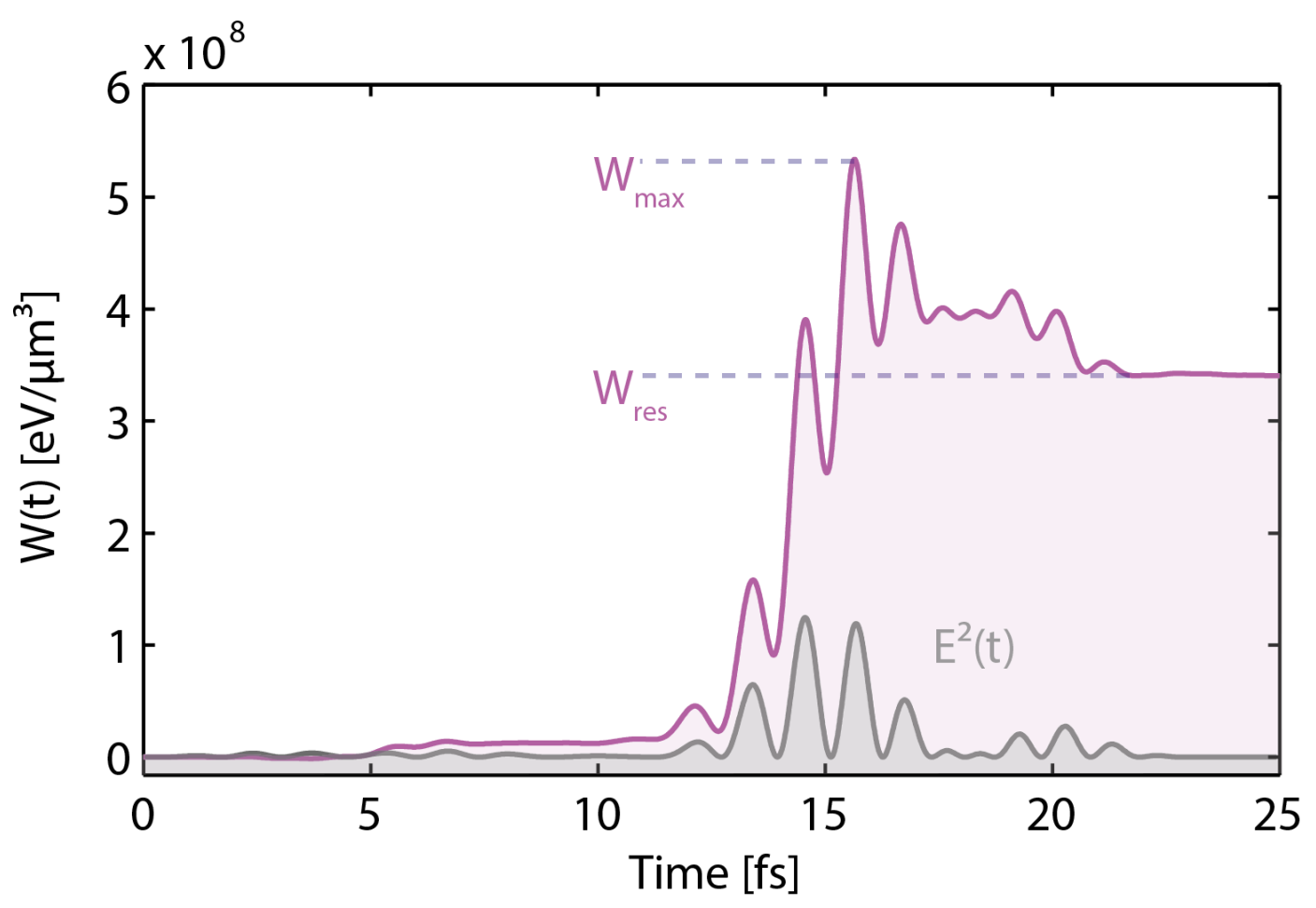

Figure 6.21: The energy density (purple line) transferred between driving field (grey line depicts the square of the electric field) and the material is extracted from Eq.(6.16).

the system and thereby transfers energy to the material. The energy flow rises with increasing field amplitude approximately until the center of the pulse. As the field strength decreases the electrons can partially return to their initial state and energy is transferred back to the field in the second half of the pulse. The slope of $W(t)$ is directly related to the shift between the strong field and the nonlinear polarization (Fig. (6.18)). $W(t)$ is calculated as the integral over the strong field and the induced current, represented by the temporal derivative of $P_{N L}(t)$ (Eqn.(6.16)). If the system follows the driving force instantaneously $P_{N L}(t)$ oscillates in phase with $E(t)$. The integration over the period of the pulse would result in zero dissipated energy. The transferred energy can only be non zero if $P_{N L}(t)$ and $E(t)$ are delayed with respect to each other. In the first half of the pulse the nonlinear polarization lags behind the driving field as energy flows to the medium. In the second half the electrons return energy to the field which reverses the direction of the shift between $P_{N L}(t)$ and $E(t)$.

The residual energy density $W_{\text {res }}=W(t \rightarrow \infty)$ deposited in the medium after the pulse vanished defines the amount of energy which is irreversibly transferred from the electric field to the material. This work is performed by the external field to excite real carriers across the bandgap into the conduction band. Their lifetime outlasts the interaction period [67]. This means that the electrons cannot return their energy to the field upon decaying and therefore cause energy dissipation. $W_{\text {res }}$ can be related to the residual real conduction band population 
$n_{\text {real }}$

$$
n_{\text {real }}=\frac{W_{\text {res }}}{E_{g}}
$$

with $E_{g} \approx 9 \mathrm{eV}$.

For an electric field with an intensity of $I=(1.3 \pm 0.1) \cdot 10^{14} \frac{\mathrm{W}}{\mathrm{cm}^{2}}$ the amount of real excited electrons is $n_{\text {real }}=(3.4 \pm 1.5) \cdot 10^{7} \frac{1}{\mu \mathrm{m}^{3}}$. This means that $0.02 \% \pm 0.01 \%$ of the valence band population is permanently excited into the conduction band during the light-matter interaction. In section 6.4.1 the fraction of excited carriers was derived from the residual phase shift between $E_{r e f}(t)$ and $E(t)$ with the Drude model. This estimation resulted in an upper limit of $0.008 \pm 0.005 \%$ excited valence band electrons after the interaction. The conduction band in fused silica is energetically very broad (approximately $10 \mathrm{eV}$ ). Electrons are not only excited into states close to the band edge but hot carriers populate the complete spectral width of the band. Therefore the estimation for $n_{\text {real }}$ computed from Eqn.(6.26) with $E_{g} \approx 9 \mathrm{eV}$ constitutes a conservative upper limit for the generated amount of real excited carriers.

In analogy to the relation between $W_{\text {res }}$ and $n_{\text {real }}$ the difference between the maximum transferred energy $W_{\text {max }}$ and $W_{\text {res }}$ can be connected to the induced virtual population [4, 68, 69]. Close to the center of the pulse the electric field performs the maximum amount of work to perturb the electronic structure of the material. Thereby the laser dressed valence band states which are fully populated in the ground state are projected onto the conduction band states. This overlap generates virtual carriers which immediately decay with decreasing field strength. When the electronic system recovers from its distortion the material returns energy to the field. The process can be considered as stimulated emission. An upper limit for the maximum amount of virtual carriers generated during the interaction is given by

$$
n_{\text {virt }}=\frac{W_{\text {max }}-W_{\text {res }}}{E_{g}}
$$

with $E_{g} \approx 9 \mathrm{eV}$. At a peak intensity of $I=(1.3 \pm 0.1) \cdot 10^{14} \frac{\mathrm{W}}{\mathrm{cm}^{2}}$ the strong field excites at most a number of virtual charges of $n_{\text {virt }}=(2.1 \pm 1) \cdot 10^{7} \frac{1}{\mathrm{~m}^{3}}$ (Fig.6.21).

Comparison of the temporal evolution of the nonlinear phase shift $\Delta \phi_{N L}(t)$ (Fig.(6.11) and $W(t)$ identifies the induced virtual population as source of the increase in optical material density throughout the pulse. As verified in the gas phase free carriers decrease the refractive index of the medium. The generated real conduction band electrons contribute negatively to the phase shift and counteract the effect of the virtual carriers. However the amount of real excited carriers is too small to have a detectable effect on $\Delta \phi_{N L}$.

The intensity dependence of $W(t)$ is investigated in Fig.(6.22). 4 individual data sets recorded in the intensity range between $0.9-1.4 \cdot 10^{14} \frac{\mathrm{W}}{\mathrm{cm}^{2}}$ are depicted.

If the material is exposed to pulses with peak intensities higher than $I>1 \cdot 10^{14} \frac{\mathrm{W}}{\mathrm{cm}^{2}}$ 


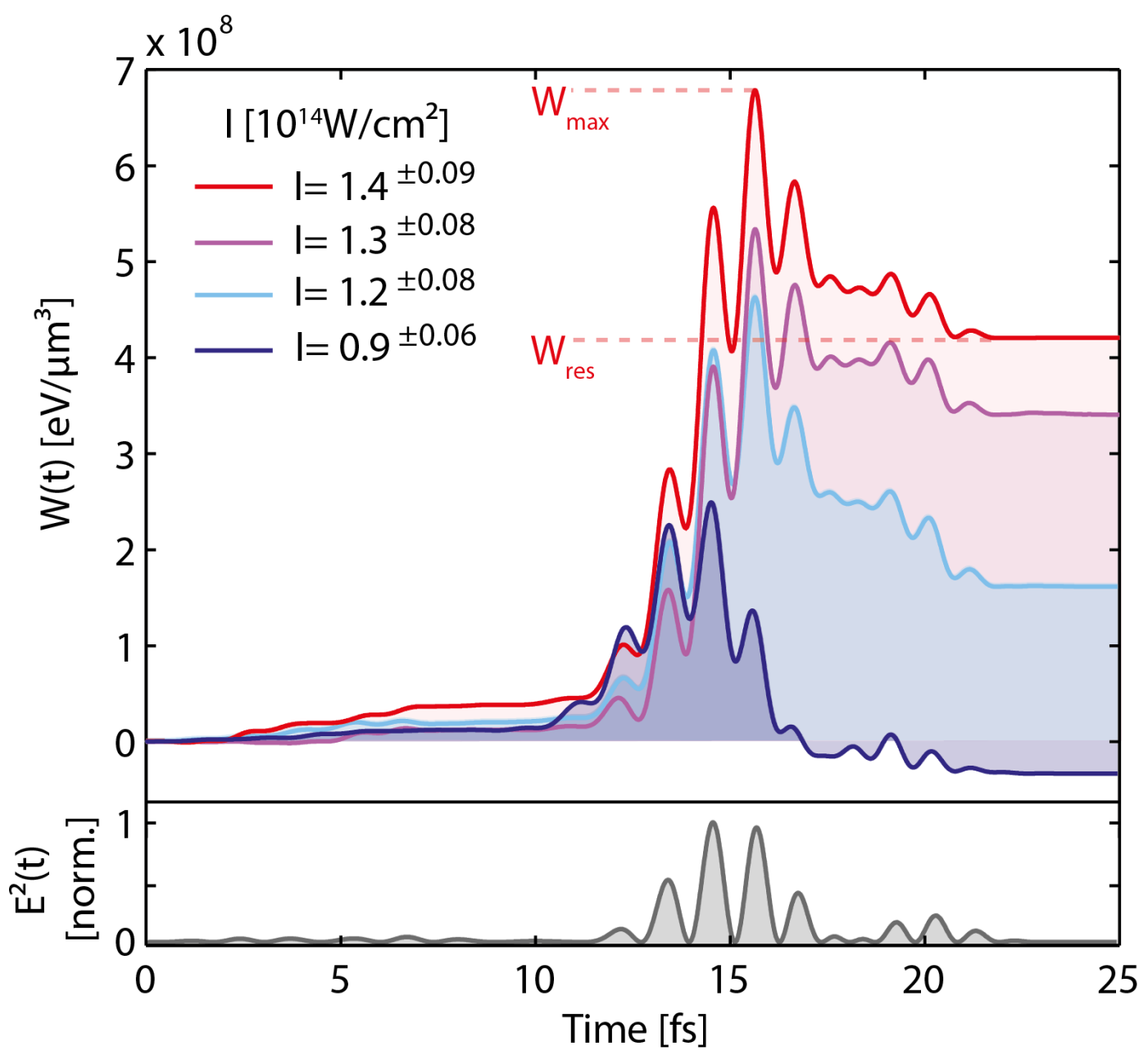

Figure 6.22: The energy density transferred between the material and the driving field for different peak intensities of the electric wave (increasing from dark blue, light blue, purple to red). The square of the electric field is indicated in the bottom panel (gray line).

the qualitative temporal profile of $W(t)$ exhibits the characteristic features described above. The generation of virtual and real carriers are the two physical processes determining the nonlinear interaction. Even at field strength close to optical breakdown no new additional mechanisms are triggered. As expected for increasing peak intensity the total amount of generated virtual and real carriers rises. At intensities below $I<1 \cdot 10^{14} \frac{\mathrm{W}}{\mathrm{cm}^{2}}$ a negligible amount of real carriers is excited in the interaction which lies below the experimental detection limit. The data set depicted in Fig. (6.22) for an intensity of $(0.9 \pm 0.06) \cdot 10^{14} \frac{\mathrm{W}}{\mathrm{cm}^{2}}$ is closest to the average over 5 scans recorded at this field strength. The results for $W_{\text {res }}$ of the individual traces scatter around zero, negative values lie within the error bar. It is interesting to investigate the intensity scaling of $W_{\text {res }}$ and $W_{\max }$ in more detail: An increase in intensity from $(0.9 \pm$ $0.06) \cdot 10^{14} \frac{\mathrm{W}}{\mathrm{cm}^{2}}$ to $(1.2 \pm 0.08) \cdot 10^{14} \frac{\mathrm{W}}{\mathrm{cm}^{2}}$ approximately doubles $W_{\text {max }}$. $W_{\text {res }}$ rises steeply from a value close to zero to a measurable amount of residual energy deposited in the material. Virtual carriers are generated during the perturbation of the energetic structure of the material 
by the strong field. The reversible polarization of the medium is described by the Kerr effect. This means that the maximum number of excited virtual carriers scales with the peak field strength to the power of 4 or the peak intensity to the power of 2 . Real carriers are excited via multiphoton absorption where the excitation rate grows proportional to $I^{5}$ or $I^{6}$ for widegap solids and optical fields. Fig.(6.23) displays the scaling of the excited real and virtual population with intensity for 5 individual measurements. The statistics of the obtained results are insufficient to confirm the predictions of the Kerr effect (light blue dashed line) and multiphoton absorption (dark blue dashed line). However the data hints to a more nonlinear increase of $n_{\text {real }}$ (dark blue circles) with intensity than for $n_{\text {virt }}$ (light blue circles).

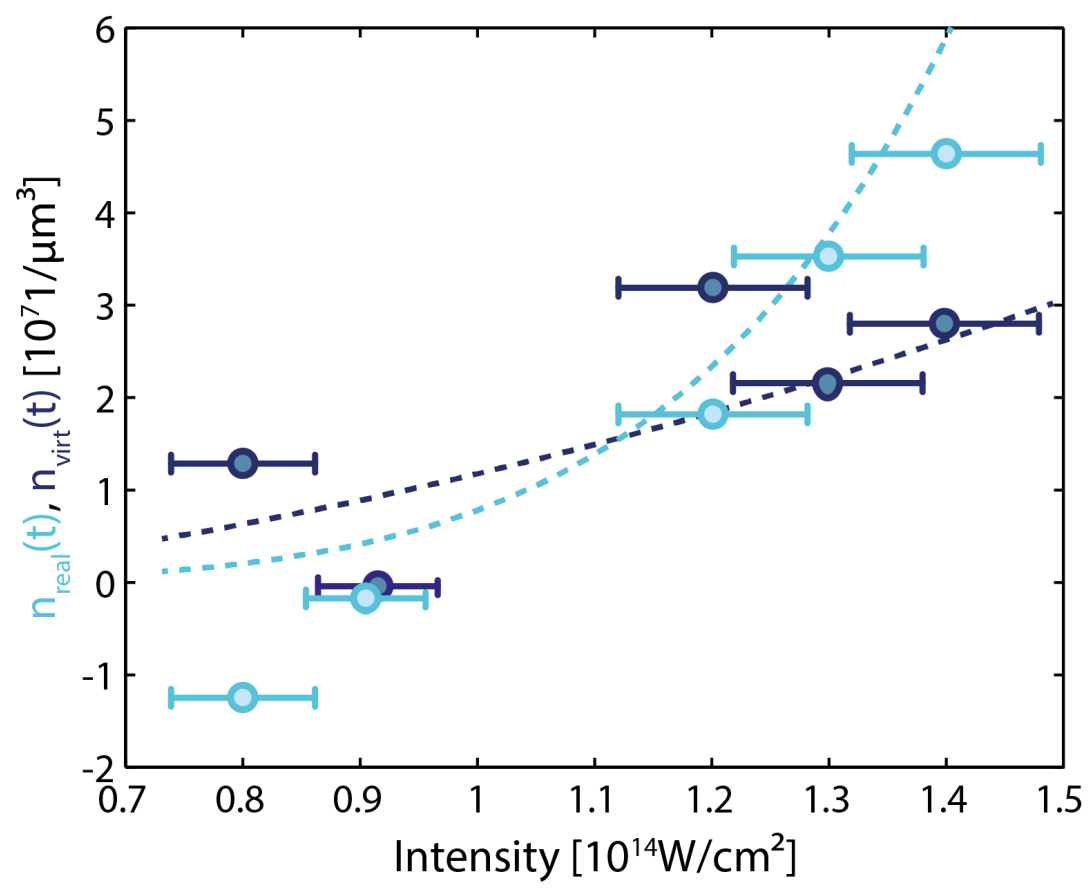

Figure 6.23: The density of the generated virtual (dark blue circles) and real (light blue circles) carries is evaluated as a function of the peak intensity of the driving field for 5 individual data sets. The scaling predicted by the Kerr effect (dashed dark blue line) and multiphoton absorption (dashed light blue line) is indicated as a guide to the eye.

The increase in absorption with rising field strength explains the intensity dependence of the evaluated response time (section 6.4.3). The value of $W(t)$ calculated from the integral in Eqn. (6.16) can only be non-zero if the polarization lags behind or precedes the driving field. The more energy is dissipated the bigger the delay between $P_{N L}(t)$ and $E(t)$. This means that the response of the electronic system is decelerated proportional to the amount of work performed on the system by the external field. 


\section{Energy Transfer Dynamics in Different Dielectrics}

The results obtained with APS in fused silica shed light onto the energy transfer dynamics in the light-matter interaction. It is possible to temporally resolve when the field performs which amount of work and how the material responds. The strong field generates real carriers which are permanently excited into the conduction band and virtual population which decays as the pulse vanishes. The amount of induced real carries defines the total energy dissipated in the interaction and scales highly nonlinear with the peak amplitude of the external driving field. The generation of virtual charges increases the optical material density and scales less nonlinear with intensity. As presented in section 6.4.2 and 6.4.3 the nonlinear modification of the refractive index and the structure of the nonlinear polarization in sapphire and fused silica exhibit very similar features. Therefore the dynamic energy flow between field and material in $\mathrm{Al}_{2} \mathrm{O}_{3}$ is expected to resemble the findings in $\mathrm{SiO}_{2}$. As explained in section 6.4.3 the sapphire samples are 2.5 times thicker than the fused silica plates, which improves the signal to noise ratio in the evaluation of $P_{N L}(t)$. Hence it should be possible to extract more quantitative information about the intensity dependence of the excitation probability for virtual and real carriers. This would provide further insight into the physical excitation mechanisms.

The transferred energy density extracted for sapphire at different peak intensities of the external field is depicted in Fig.(6.24). The temporal evolution of $W(t)$ is qualitatively very similar to the dynamics investigated in fused silica and in accordance with the observed intensity scaling of $\tau_{\text {reselec }}$. The direction of the energy flow between field and material reverses close to the center of the pulse. $W_{\text {res }}$ is related to the real conduction band population generated by the strong field (Eqn. (6.26)). At a peak intensity of $I=(8.1 \pm 0.5) \cdot 10^{13} \frac{\mathrm{W}}{\mathrm{cm}^{2}}$ $0.005 \%$ of the valence band electrons are permanently excited across the band gap in the interaction. This is in good agreement with the $0.003 \pm 0.001 \%$ estimated with the Drude model (Eqn.(2.47) from the residual phase shift after the pulse (Fig.6.15), section 6.4.3).

The generated amount of virtual and real charges rises with increasing peak intensity of the driving field. As explained in section section 6.4.3 the precise intensity scaling of this rise can be determined in a more reliable way than for the thinner fused silica samples. Even though only 4 individual measurements are considered they coincide very well with an increase of $n_{\text {virt }}$ with $I^{2}$ and a dependency of $n_{\text {real }}$ on $I^{6}$ (Fig.(6.25)). As explained for fused silica this is the scaling predicted by the Kerr nonlinearity and multiphoton absorption for wide-gap dielectrics. 


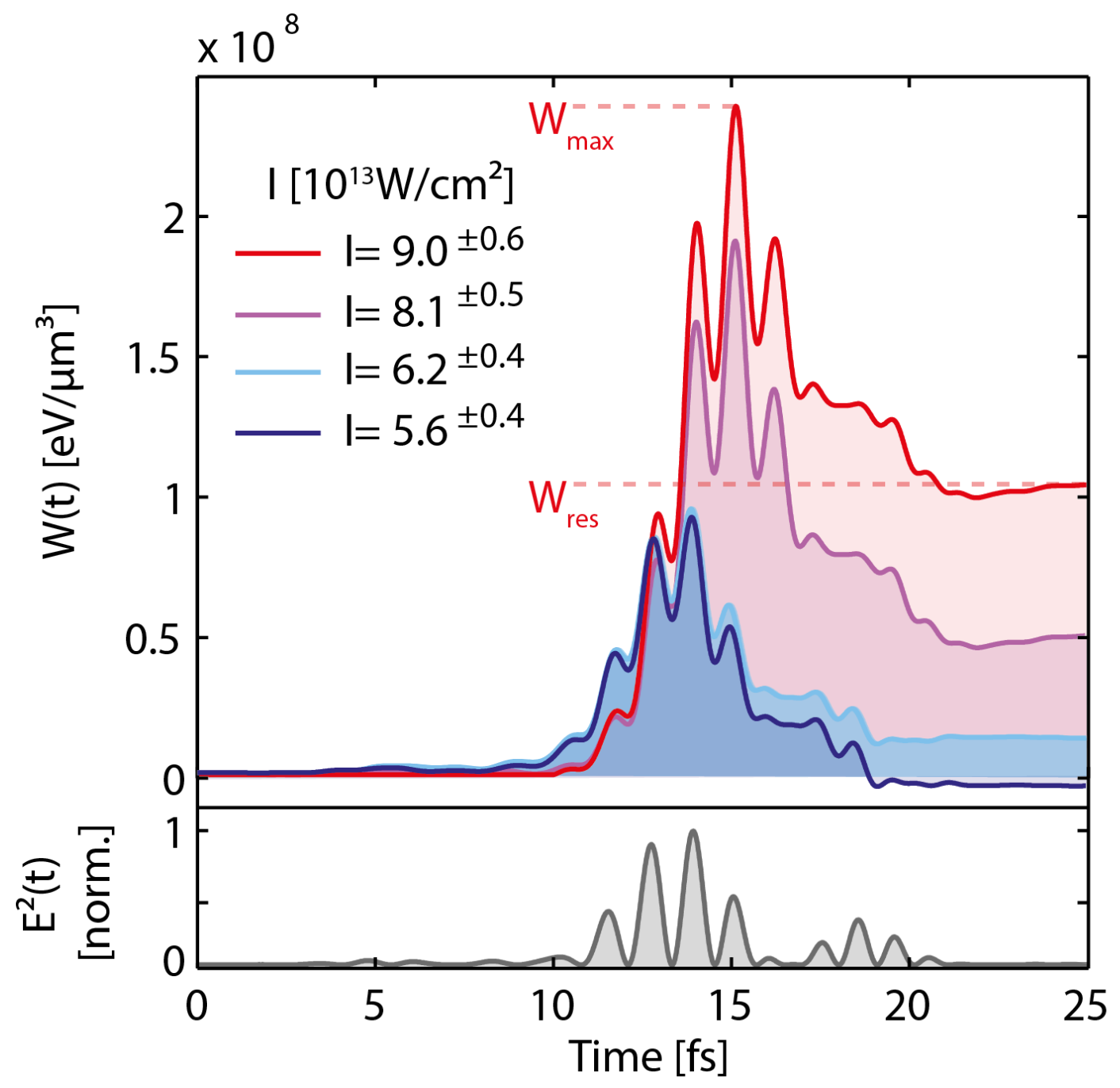

Figure 6.24: The dissipated energy density in sapphire is evaluated with Eqn.(6.16) for increasing peak intensity (from dark blue, light blue, purple to red) of the external driving field. $E^{2}(\ell / 2, t)$ is plotted as the gray line in the bottom panel). 


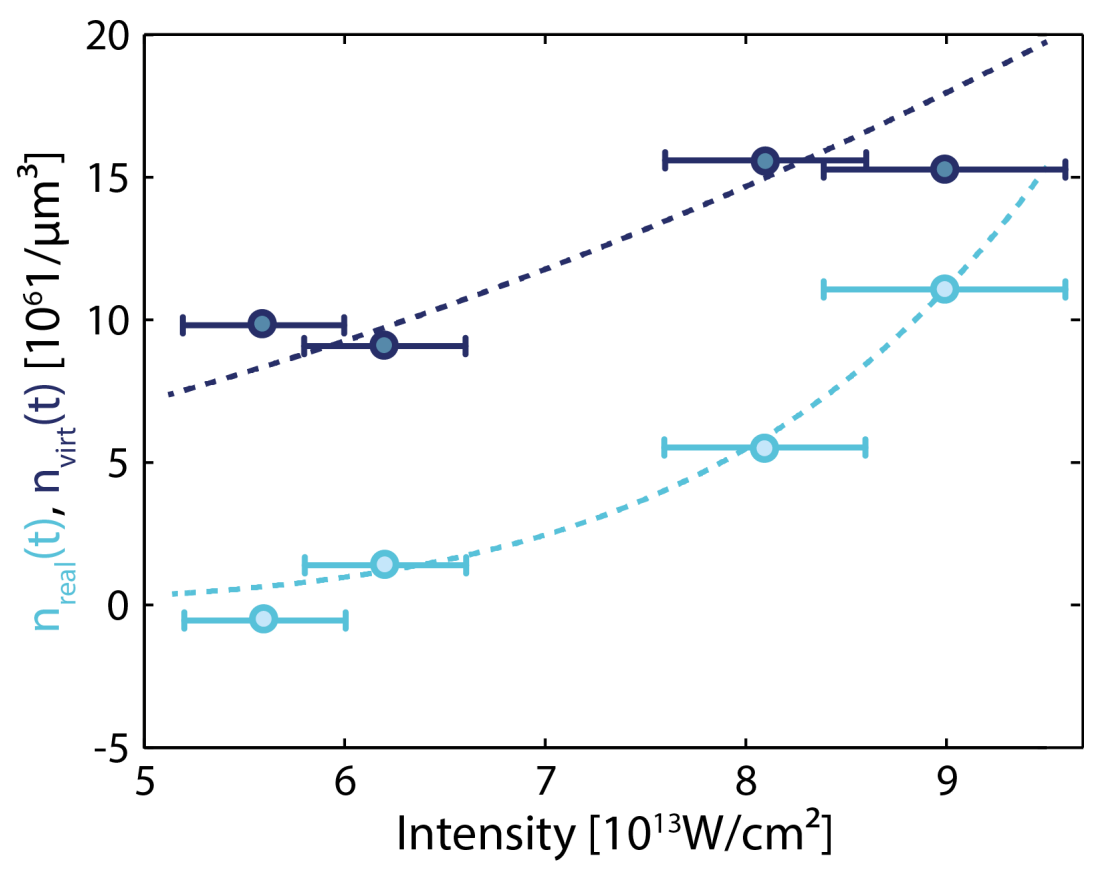

Figure 6.25: The amount of virtual (dark blue circles) and real (light blue circles) carriers generated by a field with the peak intensity $I$ in $25 \mu \mathrm{m}$ sapphire. The data is extracted from 4 representative measurements. The scaling predicted by the Kerr effect (dashed dark blue line) and multiphoton absorption (dashed light blue line) agree well with the experimental data.

The presented results demonstrate that the nonlinear energy transfer induced by intense few-cycle pulses in fused silica and sapphire exhibits the same characteristic features. Throughout the pulse both materials absorb energy with rising electric field strength which they partially return to the field when the amplitude of the driving wave decreases. This temporal evolution is caused by two physical mechanism: the maximum number of generated virtual carriers follows the Kerr prediction and a small fraction of real electrons is excited into the conduction band via multiphoton absorption.

The extracted nonlinear material response of two different insulators to ultrashort intense light pulses exhibits big qualitative and quantitative similarities. This finding strongly hints to the conclusion that the revealed physical mechanisms dominate the ultrafast strong field dynamics in all wide-gap materials.

\subsubsection{Energy Consumption of a Hypothetical Ultrafast Optical Transistor}

The main aim of the investigation of the ultrashort strong field dynamics in dielectrics is to determine whether they can be used for signal processing. The fastest state-of-the-art electronic transistors can operate at clock frequencies of $100 \mathrm{GHz}$ [5]. The results of APS prove that it is possible to reversibly modulate the optical density of dielectrics with optical few femtosecond pulses at $>100 \mathrm{THz}$ speed. One limiting factor for the performance of current electronic semiconductor devices is the energy dissipated in a switching cycle which 
leads to accumulated heat [70], [71]. The devices are downscaled in size to the sub micrometer range to decrease the switching time and minimize the heat generation. Energy dissipation will also be a critical quantity for future ultrafast optical switches. As APS provides access to the energy dissipated in the nonlinear light-matter interaction it can be benchmarked to the energy consumption of current electronic devices.

A state-of-the-art MOSFET in a CMOS inverter with a delay time of $20 \mathrm{ps}$, a gate length of $100 \mathrm{~nm}$ and a capacity between source and drain of $2.8 \mathrm{fF} / \mu \mathrm{m}^{2}$ operated with a gate voltage of $1.5 \mathrm{~V}$ dissipates in average $0.5 \mathrm{~mW}$ in one switching cycle [5]. It is $(60 \mathrm{~nm})^{2}$ big. In a very conservative approximation the complete $(60 \mathrm{~nm})^{2}$ are considered as the active area. Under this assumption the MOSFET dissipates an energy density of $3.6 \cdot 10^{8} \frac{\mathrm{eV}}{\mathrm{um}^{3}}$. In the nonlinear light-matter interaction the residual transferred energy density $W_{\text {res }}$ defines the amount of consumed energy. The energy consumption of the MOSFET is of the same order of magnitude as $W_{\text {res }}$ in fused silica in the interaction with strong fields with amplitudes bigger than $2.7 \mathrm{~V} / \AA$. This field strength corresponds to an intensity $>1.4 \cdot 10^{14} \frac{\mathrm{W}}{\mathrm{cm} \mathrm{m}^{2}}$ and is close to the optical breakdown threshold of the material (appendix 9.7). In the light-matter interaction the amount of excited virtual carriers determines the induced change in refractive index. The modification of the optical material density would be the observable manipulated for signal processing. Fields with amplitudes of $>2.7 \mathrm{~V} / \AA$ induce a maximum refractive index change of $\delta n / n>0.6 \%$. The results of APS show that $\delta n$ increases linearly with intensity whereas the number of real charges grows proportional to $I^{6}$. This means that the ratio between induced signal and energy consumption in a future high frequency optical switch grows with decreasing field strength. Fields with an intensity of $I=4.5 \cdot 10^{13} \frac{\mathrm{W}}{\mathrm{cm}^{2}}[E=2.7 \mathrm{~V} / \AA]$ still induce a maximum change of the relative refractive index of approximately $\delta n / n=0.2 \%$. The dissipated energy can be reduced by a factor of 900 to $4 \cdot 10^{5} \frac{\mathrm{eV}}{\mu \mathrm{m}^{3}}$.

It has to be considered that the volume of an optical device is limited by the beam size of the electric field. Principally the radius of the focus cannot be decreased below the diffraction limit [72]. However the increase in size compared to an electronic MOSFET can be compensated when the device is operated at intensities $I<5 \cdot 10^{13} \frac{\mathrm{W}}{\mathrm{cm}^{2}}$.

\subsubsection{Experimental Verification of the Derived Concept to Access the Non- linear Polarization}

In the derivation of Eqn. (6.10) the nonlinear polarization wave in thin samples is assumed to oscillate in phase with the driving field (Eqn.(6.7)). This approximation directly implies that the change of the electric field $\Delta \widetilde{E}_{\omega}(z)$ due to the nonlinear source term $\widetilde{P}_{\omega}^{N L}(z)$ increases linearly with propagation distance and material thickness (Eqn. (6.8)). The validity of Eqn.(6.7) can be experimentally verified by performing attosecond polarization spectroscopy with different sample thicknesses.

In addition to the study on $10 \mu m$ thick fused silica samples, the experiment is repeated under identical conditions with fused silica plates of $20 \mu \mathrm{m}$ thickness. The intensity of the strong and the reference field is adjusted to $I=(1.1 \pm 0.1) \cdot 10^{14} \frac{\mathrm{W}}{\mathrm{cm}^{2}}$ and $I_{r e f}=(6.5 \pm 0.3) \cdot 10^{12} \frac{\mathrm{W}}{\mathrm{cm}^{2}}$. 

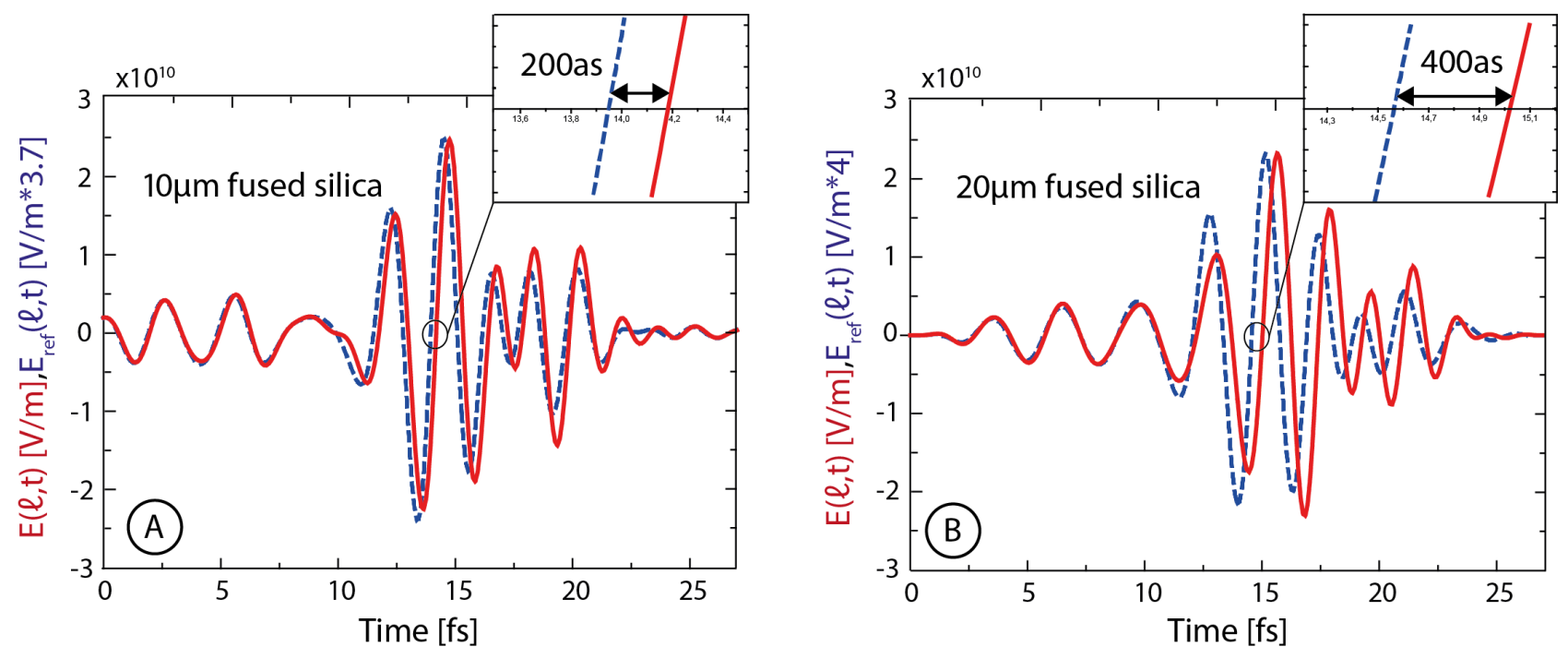

Figure 6.26: Comparison of $E(\ell, t)$ (dashed blue line) and $E_{r e f}(\ell, t)$ (red line) after the transmission through $10 \mu \mathrm{m}$ (panel A) and $20 \mu \mathrm{m}$ (panel B) fused silica. The shift between the two waves is twice as big for double the material thickness.

Fig.(6.26) compares the weak (dashed blue line) and strong field (red line) after the propagation through $10 \mu m$ (panel A) and $20 \mu m$ (panel B) material. In the thick samples the nonlinear shift between $E_{r e f}(\ell, t)$ and $E(\ell, t)$ exhibits a similar temporal profile as observed for the $10 \mu \mathrm{m}$ plates (section 6.4.1). Throughout the interaction the strong field is delayed but catches up with the linear propagated reference at the end of the pulse. In the $20 \mu \mathrm{m}$ targets the maximum accumulated phase shift is approximately twice as big as for the 10 plates. This means that the modification of the driving field $\Delta E(z, t)$ due to the nonlinearity does indeed scale with propagation length. As explained at the beginning of this section, this finding delivers direct proof for the validity of Eqn. (6.7) which describes the build up of the nonlinear polarization wave phase matched to the driving field. The approximation is on of the key assumptions in the derivation of the analytic relation between $\Delta \widetilde{E}_{\omega}$ and the nonlinear polarization (Eqn. (6.10)).

For both sample thicknesses the nonlinear polarization is evaluated from Eqn. (6.10) at the middle of the samples and compared to weak reference fields at this position in Fig. (6.27). As $P_{N L}(\ell / 2, t)$ is directly proportional to $\Delta E(z, t)$ the nonlinear shift between $P_{N L}(\ell / 2, t)$ and $E_{r e f}(\ell / 2, t)$ is also expected to grow linearly with increasing interaction length. The experimental findings support this claim as the delay between $P_{N L}(\ell / 2, t)$ (light blue line) and $E_{r e f}(\ell, t)$ (dashed dark blue line) within the experimental error is twice as big for double the material thickness.

The comparison of $P_{N L}(\ell / 2, t)$ and the strong field at the midpoint of the material confirms the robustness of the evaluation method for the electronic response time (Fig. (6.28)). For both sample thicknesses the two waves are delayed by less than $<70$ as at the zero crossing 

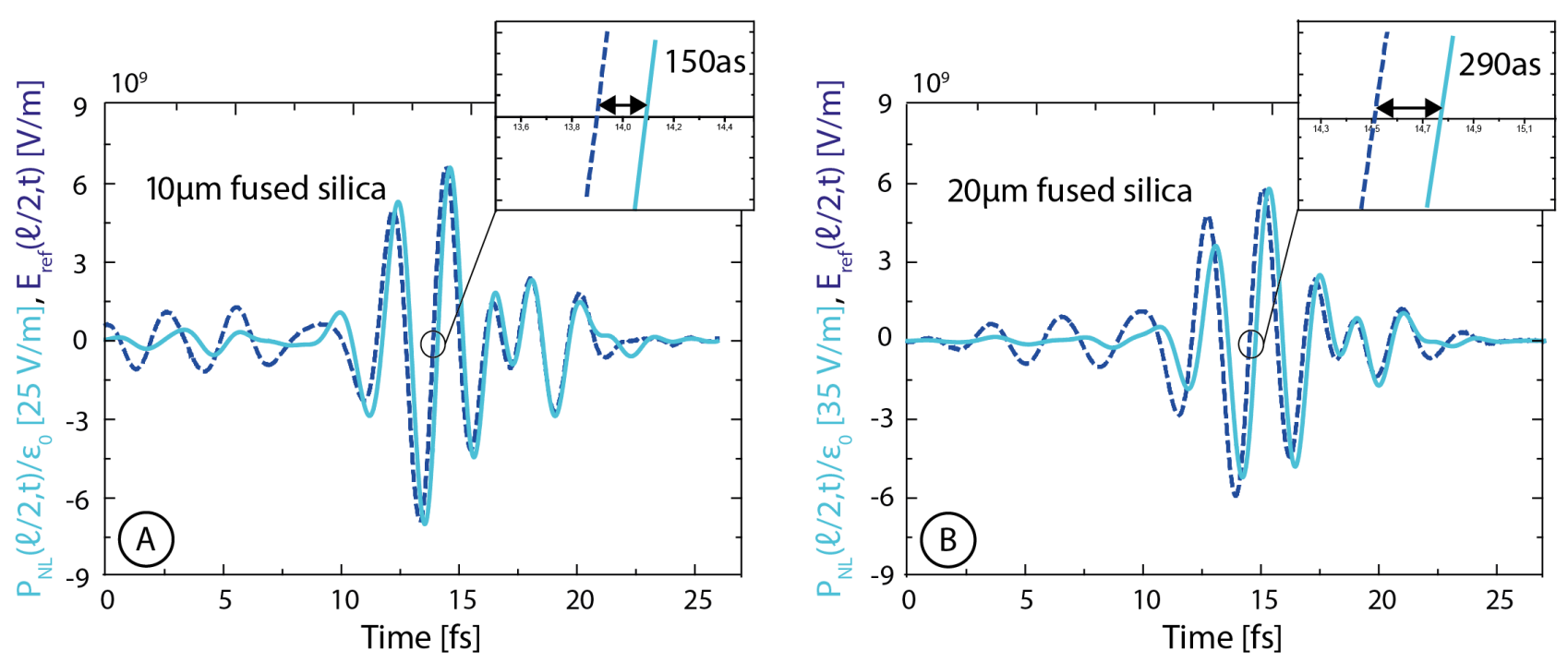

Figure 6.27: Comparison of the nonlinear polarization (light blue line) and the weak reference field (dashed blue line) at the middle of a $10 \mu \mathrm{m}$ (panel A) and $20 \mu \mathrm{m}$ (panel B) thick sample. The delay of $P_{N L}(\ell / 2, t)$ with respect to $E_{r e f}(\ell / 2, t)$ doubles with twice the interaction length.

of the central flank.

As highlighted above Eqn. 6.10 relates $\widetilde{P}_{\omega}^{N L}(z)$ and $\Delta \widetilde{E}_{\omega}(z)$ in a linear way. As $\Delta \widetilde{E}_{\omega}(z)=$ $\widetilde{E}_{\omega}(\ell)-\beta^{-1} \widetilde{E}_{\omega}^{r e f}(\ell)$ this implies a high sensitivity of the calculated nonlinear polarization on the attenuation parameter $\beta$. In the experiment the amplitude of the strong field is attenuated to record the weak reference scan $E_{r e f}(\ell, t)$. To compare the reference and strong field in Eqn. 6.10 this attenuation has to be compensated for theoretically $\beta^{-1} E_{r e f}(\ell, t)$. In experiment beta can be determined within an accuracy of $5 \%$. This variation in amplitude translates itself into a variation of the electronic material response time of 30 as.

Therefore an upper limit of the electronic response time of fused silica to the reported few-cycle pulses of $\tau_{\text {res elec }}<100$ as is claimed at intensities close to optical breakdown. 

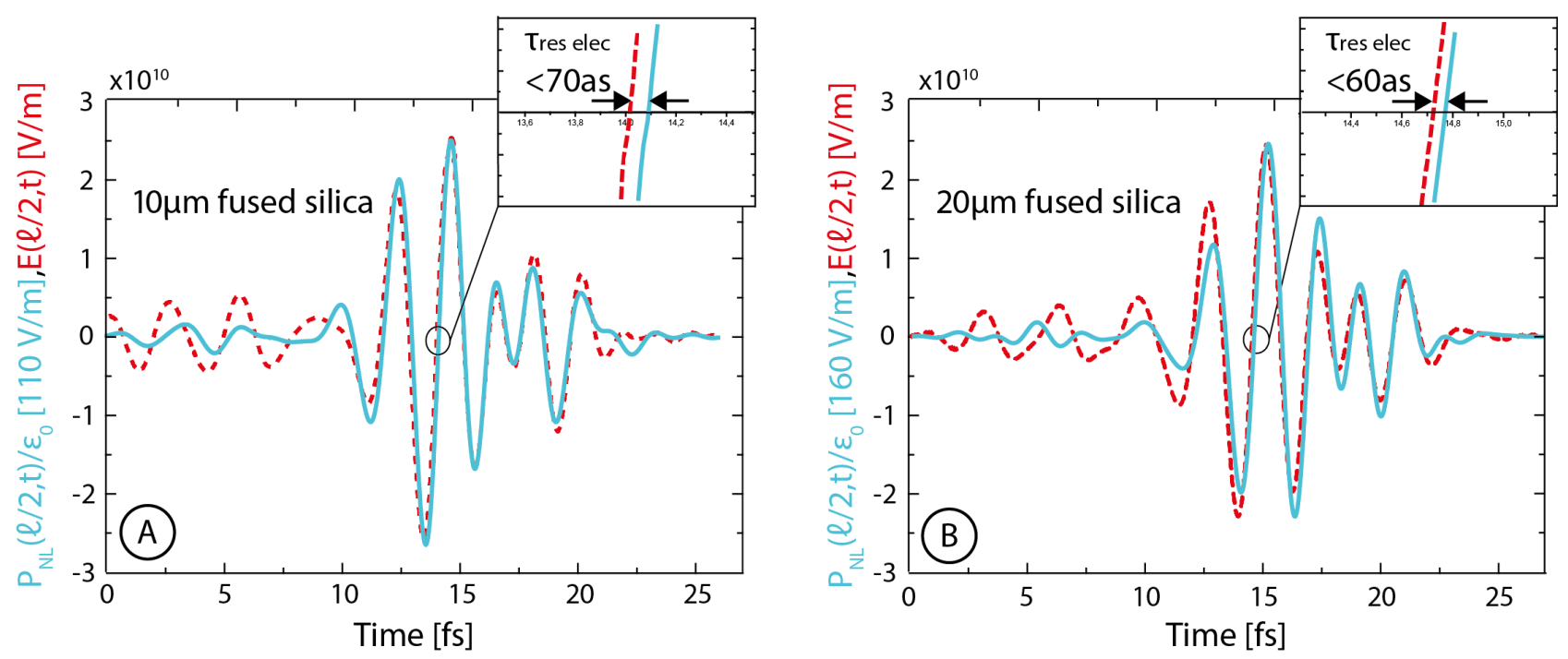

Figure 6.28: Comparison of the nonlinear polarization (light blue line) and the strong field (red dashed line) after half the propagation distance through a $10 \mu \mathrm{m}$ (panel A) and $20 \mu \mathrm{m}$ (panel B) thick fused silica plates. The comparison of the delay of $P_{N L}(\ell / 2, t)$ to $E(\ell / 2, t)$ in the zero crossing of the central flank results in the same electronic response time independent of the material thickness.

\subsection{Major Findings of APS}

Attosecond polarization spectroscopy is an experimental technique which directly facilitates the complete characterization of the nonlinear modification of the strong driving field in the interaction of intense few-cycle laser pulses with matter at petahertz frequencies. In contrast to conventional pump-probe techniques it is not restricted to the examination of individual features of the nonlinear dynamics [73]. In the presented implementation of APS the strong field functions as pump and probe field. The temporal resolution of conventional techniques is limited by the extent of the intensity envelope of the probe pulse. In APS the nonlinear interaction is resolved with pulses of a few hundred attosecond duration. As electron dynamics in solids evolve on attosecond timescales their motion can be claimed to be examined in real time.

With the developed theoretical concept the complete amplitude and phase information of the induced nonlinear polarization is accessible via an analytical relation to the experimental observables. The nonlinear material response contains the full information about the excited strong field dynamics and provides insight into the underlying physical mechanisms.

In a first step the new experimental technique is verified in the investigation of strong field ionization in gas. External fields with amplitudes above the ionization threshold generate plasma which decreases the optical density of the medium. APS can detect the acceleration of the phase velocity of the field as an increasing fraction of the neutral gas atoms is ionized during the interaction. The temporal evolution of the induced change in refractive index is directly related to the dynamic ionization rate. Therefore the amount of generated plasma at 
each point in time throughout the interaction can be determined. The evaluated nonlinear polarization is found to be dominated by the free carrier contribution. Hence it oscillates preciously out of phase with the driving field. As the ionization rate and the nonlinear polarization are accessible the expectation value of the generated electron wave packet in space can be determined directly from the measured results. It oscillates with an amplitude of less than $3 \mathrm{~nm}$.

After the successful verification of APS in gas, it is employed to investigate the strong field dynamics excited in two different insulators by intense few femtosecond pulses. In the nonlinear interaction with fused silica and sapphire qualitatively and quantitatively similar characteristic features are observed. These finding hint to an universal nonlinear response of wide-gap dielectrics to intense optical few-cycle pulses.

In the presence of the strong field the optical density of the material is increased. The Kerr prediction alone cannot describe the temporal evolution of the induced modification as the refractive index change is not directly proportional to time profile of the intensity envelope of the driving field. The reason for this discrepancy can be found in the interplay of nonlinearity and linear propagation effects for ultrashort pulses. However the change of the refractive index at the peak of the field envelope scales linearly with the maximum intensity of the driving pulse and shows no indication of saturation up to field strength close to optical breakdown. The linear increase allows to extract a proportionality factor analog to the nonlinear Kerr coefficient. Values for the Kerr index reported in literature exceed this result by up to a factor of 4 [3]. They are extracted from time integrated measurements with longer pulses under the assumption that the induced refractive index change follows the intensity envelope of the pulse.

The analysis of the strong field modification of the field envelope hints to the existence of a fast electronic and lower frequent Raman contribution to the nonlinear interaction.

The theoretical concept developed for APS provides an analytical relation between the experimental observables and the nonlinear polarization. The nonlinear polarization wave includes the complete information about the nonlinear dynamics evolving in the material. It provides access to the electronic response time of the system and the energy transfer dynamics in the interaction. The response time is found to depend on the amount of energy absorbed by the system. Hence the nonlinear material response is decelerated as the amplitude of the external field rises. For field strength close to the damage threshold of the material an upper limit of $\tau_{\text {resp elec }}<100$ as can be determined.

With the knowledge of the nonlinear polarization the dynamics of the energy transfer between field and material can be accessed. When the field amplitude increases at the beginning of the pulse the external field performs work to modify the energetic structure of the electronic system. Hence energy is transferred from the field to the material. With decreasing field strength part of the electrons return to their equilibrium position and give back energy to the medium. Only the energy which is permanently absorbed is dissipated in the generation of real carriers via multiphoton absorption. In the presence of the field laser dressed valence band states are projected onto the conduction band. This leads to the non permanent excitation of virtual carriers [4]. They vanish with decreasing amplitude of the driving 
wave and thereby return energy to the field. This process can be considered as stimulated emission. The virtual population is related to the detected increase of the optical material density. Their maximum number scales according to the Kerr effect. At field strength close to optical breakdown less than $0.01 \%$ of the valence band electrons are permanently excited as real carries into the conduction band by the external field. This generated conduction band population decreases the refractive index of the material. However their number is too small to have an experimentally detectable effect.

As the real carriers are generated in the highly nonlinear process of multiphoton absorption, their excitation probability increases faster with the peak intensity of the driving field than the generation of virtual charges. Their maximum number follows the Kerr prediction. This means that the amount of energy dissipated in the interaction scales more nonlinearly with the amplitude of the driving field than the induced refractive index change. These findings are of high relevance for the application of nonlinear optical switches in signal processing at $>100 \mathrm{THz}$ frequencies. The energy density consumed in the interaction can be decreased several orders of magnitude below the characteristic values of state-of-the-art electronic $\mathrm{GHz}$ devices if the dielectric is operated with the smallest detectable signal at field intensities below $I<5 \cdot 10^{13} \frac{\mathrm{W}}{\mathrm{cm}^{2}}$. 


\section{Chapter 7}

\section{Outlook}

\subsection{Wave Form Synthesis}

The results presented in the previous chapters point out that APS is a suitable technique for the time resolved investigation of the nonlinear dynamics intense few-cycle pulses induce in matter. Its potential can be further exploited if another degree of freedom is accessible: by employing few-cycle pulses with a modifiable temporal envelope, the dependence of the nonlinearity not only on the peak intensity but on the shape of the waveform can be explored. It would be interesting to investigate the sensitivity of the temporal evolution of the nonlinear phase difference $\Delta \phi_{N L}(t)$ on the temporal composition of the driving field. The variation of the response time and energy dissipation dynamics could be examined in more detail. Like this it would be possible to optimize the temporal profile of the waveform for the maximum ratio between generated virtual and real carriers. The tailored field offers the best compromise between induced change in optical density and dissipated energy which are the critical parameters for future application in signal processing.

The approach pursued here to generate few-cycle pulses with a defined non Gaussian time profile is to spatially divide the broadband spectrum into two narrow band parts. The phase of the light field in each channel is individually manipulated with customized chirped mirrors (appendix 9.2, faricated by Olga Razskazovskaya). Afterwards the different pulses are recombined. By varying the delay between them, various waveforms can be tailored. As displayed in Fig.(7.1) the spectrum is divided at $600 \mathrm{~nm}$ into a blue channel extending from $400-600 \mathrm{~nm}$ and a red channel from $600-1000 \mathrm{~nm}$. The technical details of the setup are specified in appendix 9.9. In the following the temporal characteristics of the pulses in the individual arms are presented.

As the pulse intensity in the blue channel is too low to efficiently drive the highly nonlinear process of high harmonic generation (appendix 9.3) attosecond streaking is not well suited (appendix 9.4) for the characterization of the pulses in the individual arms. The electric

field is measured with the technique of transient grating frequency resolved optical gating (TG FROG, appendix 9.5] [46]. It cannot determine the absolute value of the phase of the electric field. This means that the carrier envelope phase and the absolute arrival time of the 

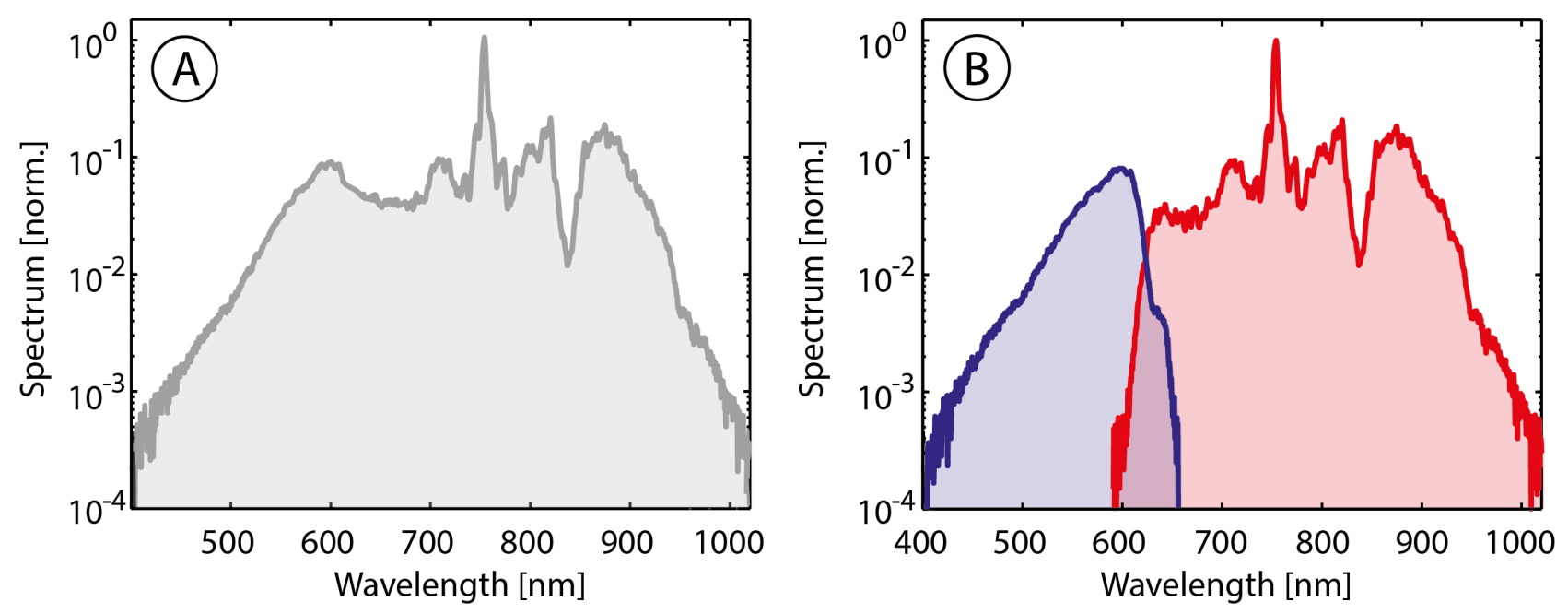

Figure 7.1: The spectrum of the few-cycle pulse (Panel A, appendix 9.1.2) is divided into the blue and red channel channel (Panel B) of the wave form synthesizer.

pulse is not accessible (appendix 9.5). However the TG FROG technique provides complete information about the amplitude and relative phase of the electric field and hence allows to extract the pulse duration with comparable small experimental effort.

\section{Blue Channel of the WFS}

The recorded (Panel A) and reconstructed (Panel B) TG FROG spectrogram of the pulse exciting the blue arm of the wave form synthesizer is depicted in Fig.(7.2). Fig.(7.3) displays the retrieved spectral intensity (dark blue line) and phase (light blue line). The structure of the spectral phase between $400-550 \mathrm{~nm}$ of the uncompressed pulse before compensation by the chirped mirrors is highly sophisticated. Without the wave form synthesizer the complete pulse spectrum is compressed with one set of chirped dielectric mirrors (appendix 9.1.2). Their design has to be chosen as the best compromise between compensating higher order nonlinearities of the spectral phase and maintaining the highest reflectivity throughout the whole spectrum (appendix 9.5). Therefore it is not possible to consider the complex phase structure in the wavelength range below $500 \mathrm{~nm}$ in great detail. It is largely ignored as it contains less than $<10 \%$ of the pulse energy. For the wave form synthesizer the spectrum is divided and the bandwidth in the individual channels decreased. Like this the phase nonlinearities can be compensated more individually with the chirped mirror design. As visible in Fig.(7.3) the spectrum is successfully compressed down to $450 \mathrm{~nm}$. This results in a pulse duration of $\tau_{F W H M}=7.6 \mathrm{fs}$ which is close to the Fourier limit of $\tau_{F L}=6.1 \mathrm{fs}$. 

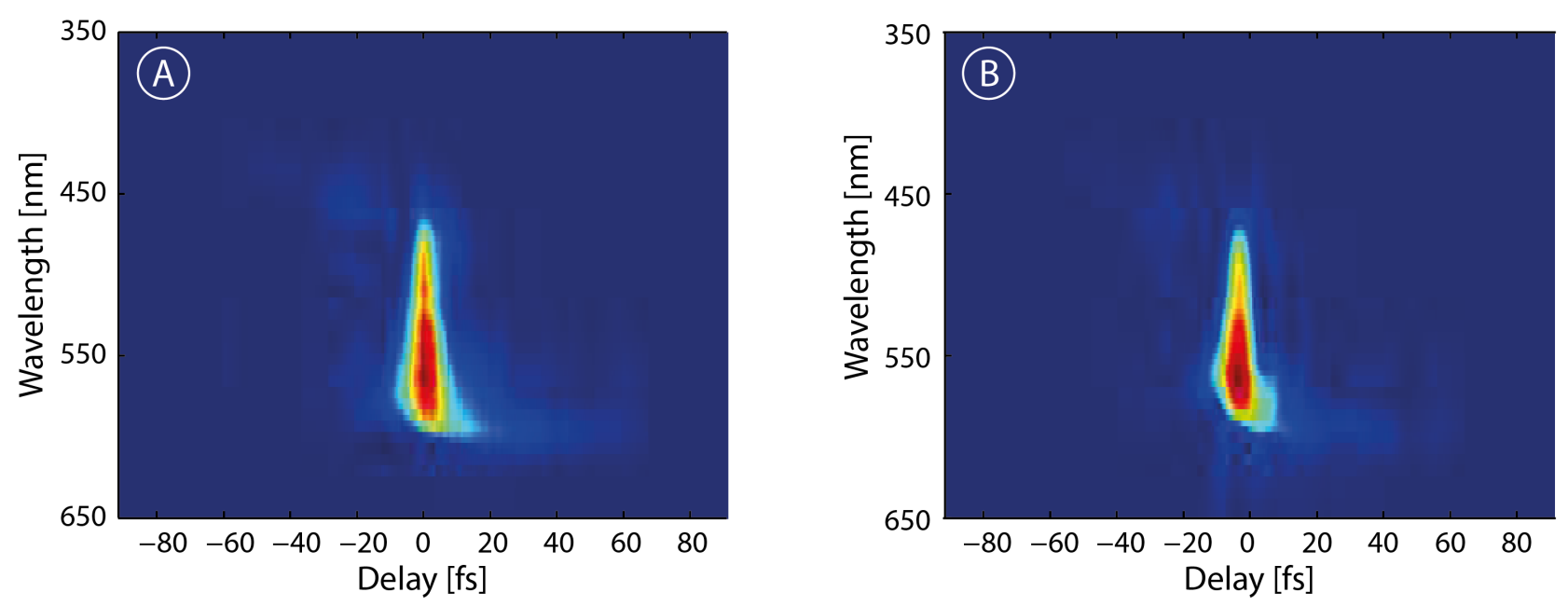

Figure 7.2: Panel A: The recorded TG FROG spectrogram of the blue channel. Panel B: The retrieved trace which deviates by a FROG error of $8 \cdot 10^{-3}[74]$.

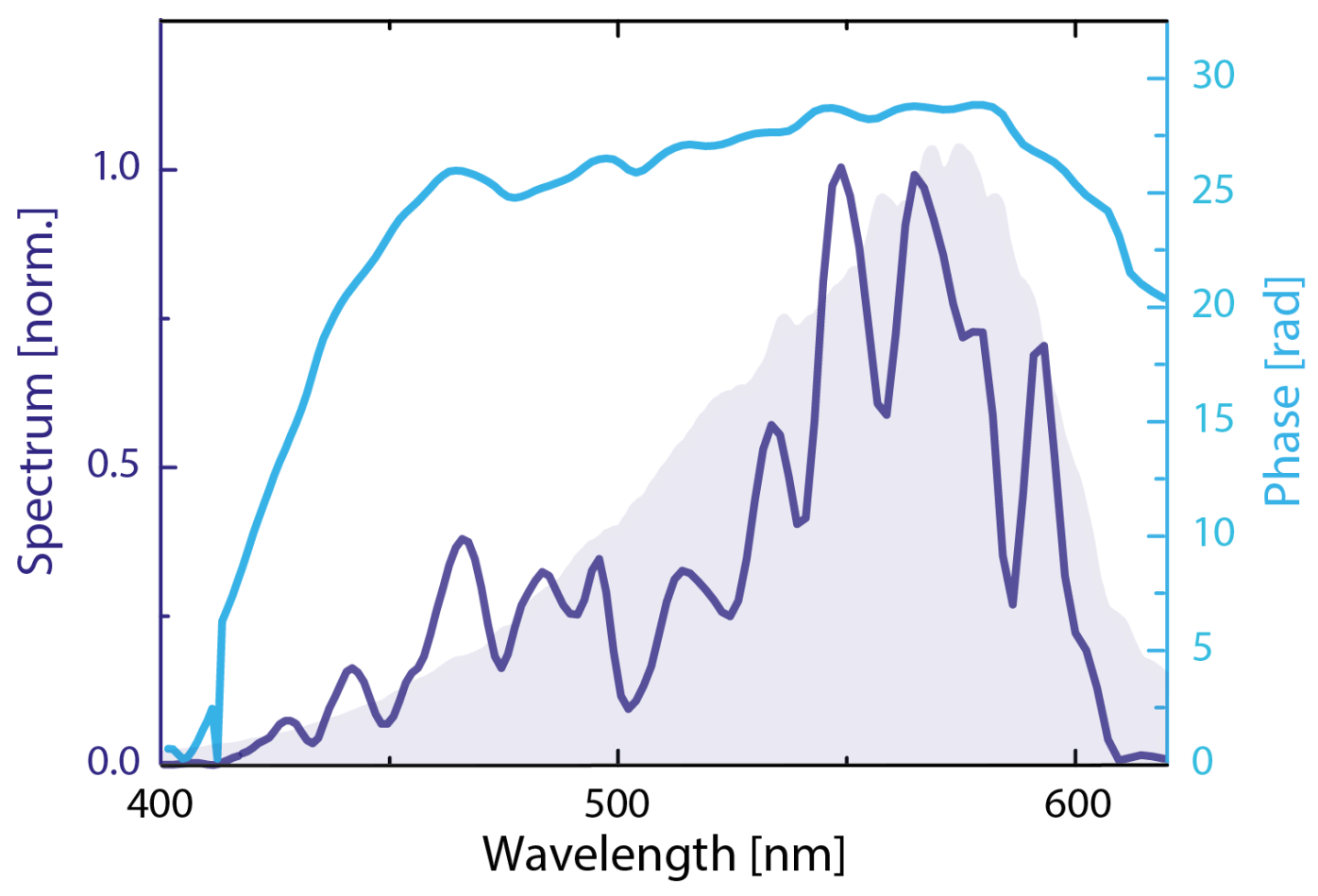

Figure 7.3: The spectral intensity (dark blue line) and phase (light blue line) extracted from the TG FROG spectrogram depicted in Fig.(7.2). The measured pulse spectrum is indicated by the transparent dark blue area. 


\section{Red Channel of the WFS}

The TG FROG spectrogram, extracted spectral intensity and phase of the red channel are depicted in Fig.(7.4) and Fig.(7.5). It is apparent that the spectral phase does hardly depended on frequency over the entire bandwidth. Hence the pulse is compressed to a duration of $\tau_{F W H M}=6.1 \mathrm{fs}$ which is only slightly above the Fourier limit of $\tau_{F L}=5.8 f \mathrm{~s}$.
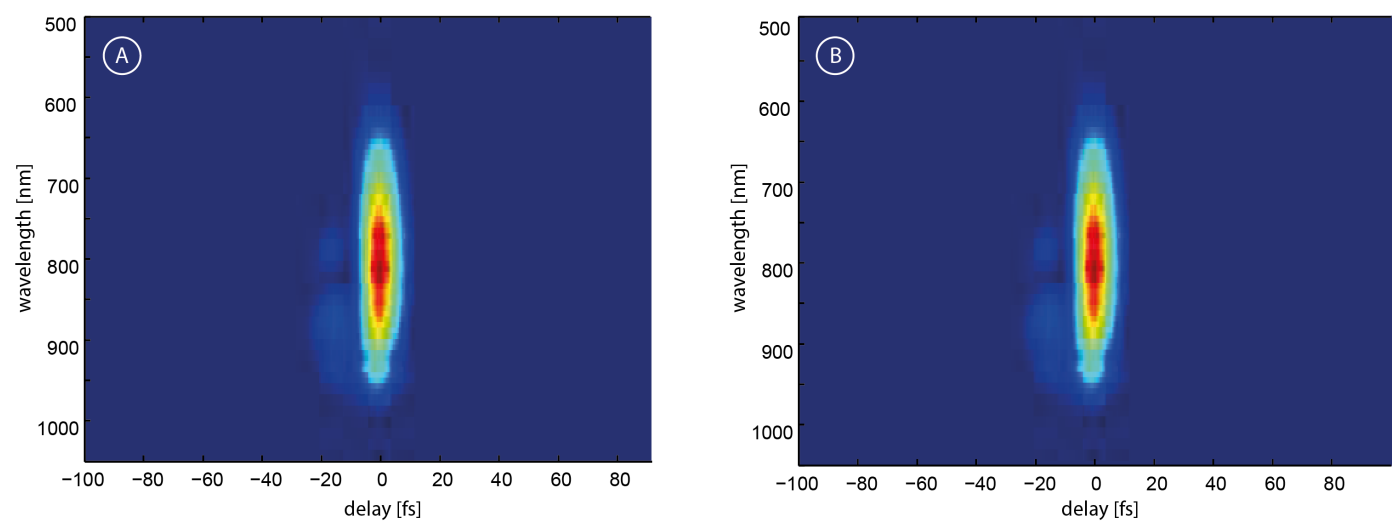

Figure 7.4: The recorded (Panel A) and retrieved (Panel B) spectrogram of the red channel. The FROG error measures the derivation of the reconstructed and measured trace and is $3 \cdot 10^{-3}$.

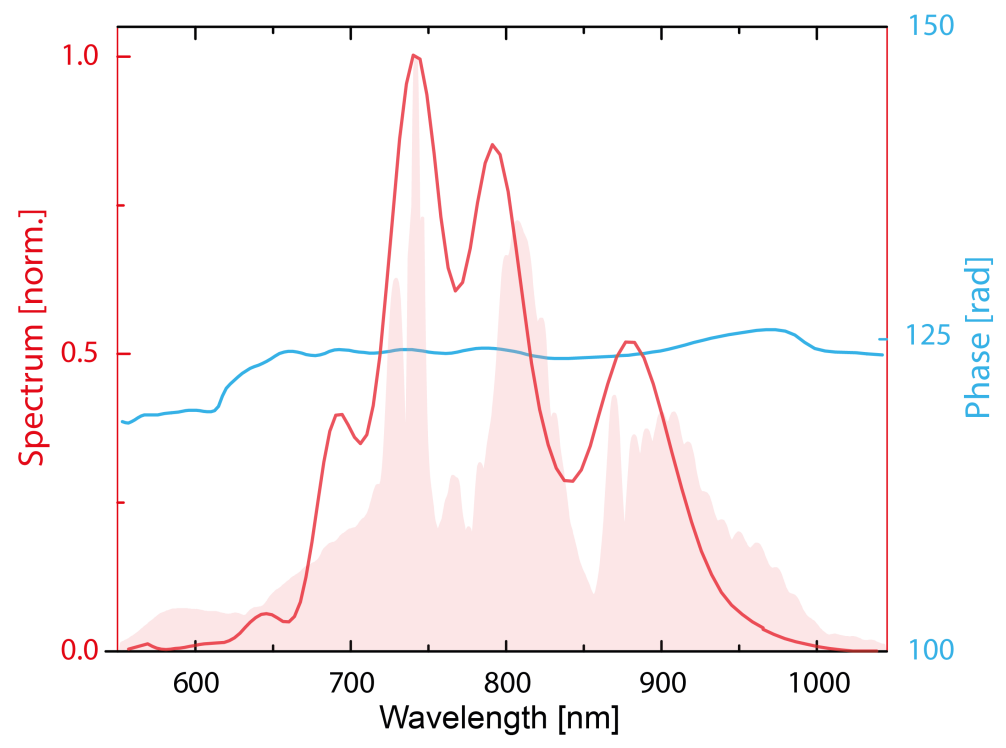

Figure 7.5: The spectral intensity (red line) and phase (blue line) retrieved from the TG FROG spectrogram depicted in Fig.(7.4). The measured spectrum is displayed as the transparent red area. 


\section{Synthesized Waveforms}

Recombination of the compressed pulses out of the two arms results in a sub 4 fs fewcycle pulse. As the phase nonlinearity below $500 \mathrm{~nm}$ is compensated to a higher degree than without the synthesizer (appendix 9.5) the contrast before and after the main field can be improved. The additional degree of freedom of delay between the channels enables the synthesis of new waveforms. The parameter facilitates more variations than sole modification of the carrier envelope phase (Fig.(9.14)). A few theoretical examples of possible tailored fields are depicted in Fig.(7.6).
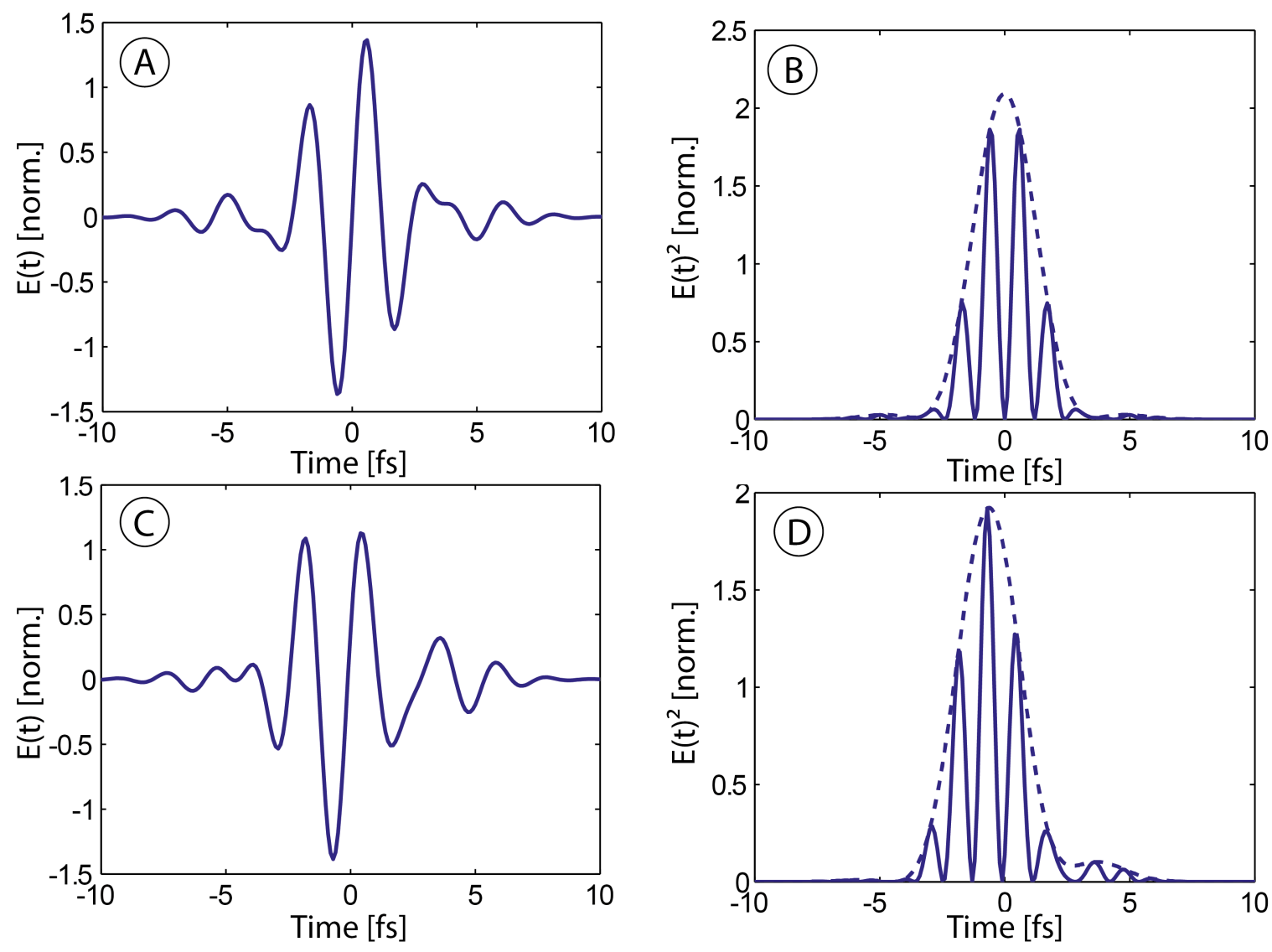

Figure 7.6: Theoretically simulated synthesized waveforms with a pulse duration of less than $\tau_{F W H M}<4 f s$ after recombination of the two channels of the waveform synthesizer. Panel A,B: For a sine pulse the oscillations preceding and succeeding the main pulse and the contrast within the subcycles can be improved. Panel C,D: It is possible to tailor a cosine pulse with a particular steep rise of the electric field within the first subcycle.

Currently there is no experimental data of the combined channels of the synthesizer available as the setup is not yet optimized for interferometric stability. Despite a water cooled solid vibration low construction is designed to attenuate mechanical oscillations and prevent thermal extension of the components. As soon as this setup is manufactured the waveform 
synthesizer will be ready for operation to tailor waveforms which will be used in the APS experiments.

\subsection{Investigation of Semiconductor Materials with APS}

In the work presented in this theses APS is used to investigate the ultrafast strong field dynamics in dielectrics. The experimental concept is not limited to any material class, but can also be employed for the examination of semi conductors. In 2014 the strong field dynamics in silicon were examined in a transient absorption scheme identical to the method described in section 4[75]. It was possible to experimentally resolve the generation of real conduction band population by an external few femtosecond pulse in real time. However the signal to noise ratio was not sufficient to extract the signature of the induced transient virtual carriers predicted by theory. As the noise level in APS is considerably improved compared to the transient absorption technique chances are high that it is possible to access the complete energy transfer dynamics in semiconductor materials.

\subsection{Extension of APS to Broadband Probe Fields}

In the current implementation of APS the strong optical few-cycle pulse functions as pump and probe field. The technique can be extended by replacing the VIS/NIR probe pulse with a weak field in the short wavelength range. To do so the spectrum is broadened by for example generating the third harmonic of the fundamental beam in a nonlinear conversion process (section 2.4). The UV photons probe strong field modification of semiconductors and dielectrics more directly as they possesses enough energy to excite electrons across the bandgap in a one or two photon process. As long as the amplitude of the probe field is kept low enough its nonlinear interaction with the material can be considered in subsequent data analysis. A possible experimental setup for such a VIS/NIR pump UV probe APS setup is depicted in Fig.(7.7). Before the sample both pulses are reflected off a rotatable mirror which is coated with different dichroic segments: all of them uniformly possess maximum reflectivity for the UV part of the spectrum, whereas the long wavelength can be variably attenuated. Like this the intensity of the pump beam can be varied while the probe field remains unaltered. After transmission through the sample the pump field is entirely suppressed by another set of dichroic mirrors. Only the remaining pump field is temporally characterized for example in an attosecond streak camera (appendix 9.4) [76]. Again a reference scan, where the sample is exposed to a weak pump field, is compared to the strong field measurement. This experimental scheme possesses attosecond resolution independent of the temporal extent of the UV pulses. A probe pulse with a duration that slightly extends the pump field could provide information about triggered dynamics evolving on longer timescales. The strong field modifications of the energetic material structure are expected to affect the amplitude and the phase structure of the probe pulse. This could facilitate the investigation of the Franz-Keldysh effect with attosecond resolution [77]. 


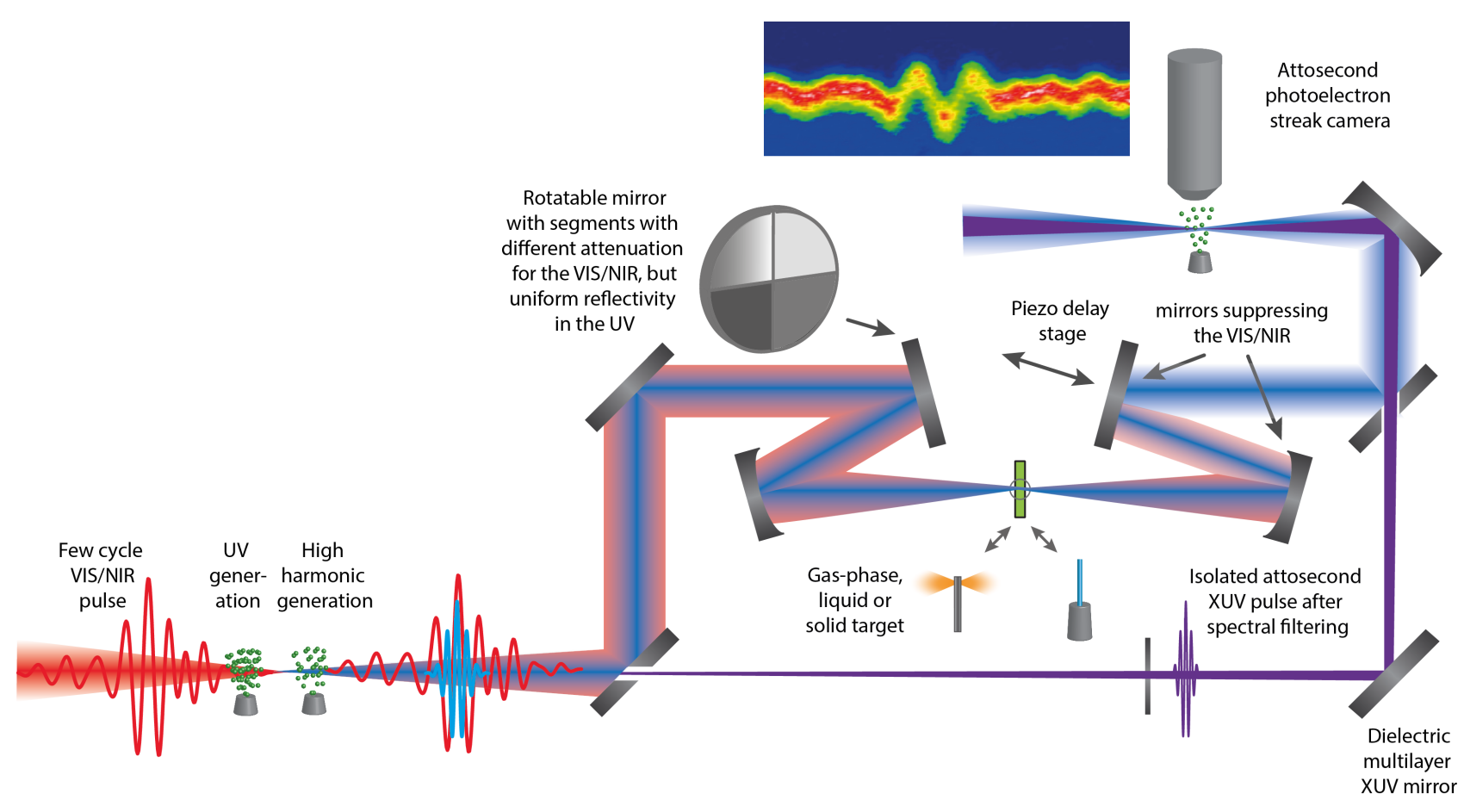

Figure 7.7: Schematic setup of APS with a strong pump field in the VIS/NIR and a separate UV probe. 


\section{Chapter 8}

\section{Conclusion}

The aim of this thesis is to examine whether the optical properties of dielectrics can be manipulated with intense few femtosecond laser pulses for future application in ultrafast signal processing. The temporal evolution of the strong field dynamics triggered by ultrashort electric fields in insulators is largely unexplored. It is not clear whether they possess suitable characteristics which can be exploited for optical high frequency switches. To estimate the feasibility of the approach the two basic questions about the nonlinear light-matter interaction raised in section 1 have to be answered:

- How fast does the material react to the external strong field and how quickly can it follow the driving wave?

- How big is the reversible contribution to the nonlinear material response compared to the irreversible part and is it possible to optimize the ratio between them for neardissipation-free optical signal processing?

The physical quantity which provides answers to these questions is the nonlinear material polarization. It is the nonlinear wave emitted by the dipoles which are excited in the medium by the strong field. Hence it contains the comprehensive information about the induced dynamics. Throughout this thesis different experimental approaches are pursued to access the nonlinear polarization.

In a first step, the nonlinear Kerr coefficient is examined with time integrated techniques. These measurements yield a considerably smaller $n_{2}$ than the values reported in literature for longer pulses [3]. To understand the physical origin of this discrepancy an experimental method which provides attosecond time resolution is developed: Attosecond Transient Absorption Spectroscopy can provide first answers to the questions raised above. The absorptivity of XUV radiation in dielectrics is found to be modified in the presence of the strong field. It is modulated and spectrally shifted with twice the laser frequency which proves that the underlying dynamics are induced by the carrier wave. The effect vanishes together with the driving field. To extend the understanding of the observed phenomena the influence of the strong field interaction on the amplitude of the reflected strong field 
is explored. It is found to increase highly nonlinearly with the intensity of the strong field at field strengths close to material breakdown. A nonlinear autocorrelation measurement reveals that the strong field modification is again reversible on femtosecond timescales.

The two experiments independently examine the influence of the strong field on two different quantities: the XUV absorptivity and the reflectivity of the strong field. The modification of both observables vanishes with the field within femtoseconds. However the obtained results do not provide direct insight into the nonlinear dynamics induced in the material, which are the reason for the alteration of the experimental observables. A complex theoretical model attempts to explain the underlying physics and extract the nonlinear material polarization. Nevertheless the experimental results are insufficient to explicitly verify the theoretical approach. Furthermore the signal-to-noise ratio of the extracted polarization is too low to reliably draw further conclusions from it.

The crucial step to the comprehensive understanding of the ultrafast strong field dynamics is the development of the technique of Attosecond Polarization Sampling. It is not restricted to the examination of distinct features of the nonlinear interaction but facilitates the complete characterization of the modified strong field with attosecond resolution. The full amplitude and phase information of the nonlinear polarization is accessible via an analytic relation to the experimental observables.

APS reveals that virtual carriers are generated in the presence of the strong field [4]. When the electric field strength rises throughout the pulse the electronic properties of the system are increasingly distorted. Energy flows from the external field to the medium to modify its energetic structure. The laser dressed valence band states are projected onto the conduction band levels and generate virtual population. With decreasing field strength the system returns to its equilibrium state and the energy which was transferred flows back to the field. The virtual charges decay via stimulated emission. In total, no energy is dissipated in the transient process.

The maximum amount of generated virtual carriers rises proportionally to the square of the intensity of the external field. This is the scaling predicted by the Kerr nonlinearity. In the presence of the strong field the optical material density is increased. The amplitude of the refractive index change at the peak of the field envelope also increases according to the Kerr proportionality. The virtual carriers and the modification of the optical density do not only exhibit the same intensity scaling, but also appear and vanish immediately with the driving field. Therefore the virtual carriers are identified as source of the induced refractive index change. These findings are very interesting in regard of future application: the refractive index of dielectrics can be predictably and reversibly modulated with strong fields on femtosecond time scales. This means that it can be used as a signal in optical high frequency switches.

Depending on the peak field strength of the external wave and the maximum amount of energy transferred between field and material the response time of the system is found to be delayed. At field strengths close to optical breakdown the electronic properties of the dielectric respond within $<100$ as. The response time is accelerated with decreasing intensity of the driving field. 
The virtual carriers are found to be generated and eliminated with the driving field. Additionally, real carriers are excited into the conduction band via multiphoton absorption throughout the interaction. Their negative contribution to the refractive index is negligibly small. However they determine the amount of energy dissipated in the light-matter interaction as their lifetime exceeds the duration of the driving pulse. This means that they cannot return the energy expended in their excitation to the field. In the interaction of optical $50 \mathrm{fs}$ pulses with fused silica a decay time of approximately $200 \mathrm{fs}$ is determined for the generated real conduction band electrons [67]. In the current implementation of APS, where the ultrashort strong field functions as pump and probe beam, this parameter cannot be determined for the excitation with sub-4-fs pulses. As described in section7.3 the APS scheme will be extended by replacing the probe with a UV pulse. If its duration slightly exceeds the pump pulse the dynamics evolving after the strong field interaction can be investigated.

The experimental findings show that the generation of real carriers grows more quickly with the intensity of the external field than the induced modification of the refractive material index. This means that the energy consumption of an ultrafast optical switching device can be optimized by choosing the smallest external field amplitude which induces a detectable signal. With this method the energy density dissipated in one switching cycle can be reduced significantly below the parameters of transistors in current semiconductor technology [5].

The presented findings show that the idea of manipulating dielectrics with few femtosecond strong fields is a valid approach in future ultrafast signal processing. The two questions raised above can be answered and show that wide-gap solids fulfill all the fundamental requirements to function as optical $>100 \mathrm{THz}$ switches: their electronic properties react within $<100$ as and can follow a driving force reversibly at $\mathrm{PHz}$ frequencies. Only a negligible number of carriers remain excited after the interaction. Their amount and therefore the dissipated energy can be minimized by correct choice of the amplitude of the driving field. 


\section{Chapter 9}

\section{Appendix}

\subsection{Laser System}

\subsubsection{Laser System Emitting Few Cycle Pulses with 500 $\mu$ J Pulse Energy}

The laser system used for the APS experiments in solids (section 6.4), the time integrated measurement of the nonlinear Kerr coefficient (section 3) and the damage detection (section 9.7) consists of three major components: the oscillator, a chirped pulse amplifier and a neon filled hollow core fiber with negatively chirped dielectric multilayer mirrors (appendix 9.2). The system emits sub-4-fs pulses in the VIS/IR (Fig.(6.4) ) with a pulse energy of approximately $500 \mu \mathrm{J}$ at a repetition rate of $3 \mathrm{kHz}$. The spectrum displayed in Fig.(9.1) extends from $\lambda=450-1050 \mathrm{~nm}$ with a central wavelength of $\lambda_{0}=780 \mathrm{~nm}$ and supports a Fourier limited pulse duration of $\tau_{F L}=3.2$ fs [78].

\section{Oscillator}

The gain medium in the oscillator is a Ti:Sa crystal cut at Brewster's angle in order to eliminate reflection losses. It is pumped by a frequency doubled $\mathrm{Nd}: \mathrm{YVO}_{4}$ laser with an average power output of $P=4.35 \mathrm{~W}$ at a wavelength of $\lambda=532 \mathrm{~nm}$ which is focused by a lens into the crystal. The pump power can be varied with an acoustic-optical modulator. The cavity is formed by high reflecting mirrors. For precise dispersion control two wedged fused silica plates are positioned in the beam path. The size of the pump laser spot in the crystal is matched to the continuous wave mode of the IR beam in the oscillator, however the Gaussian intensity profile generates increasing occupation inversion towards the center of the pump focus. Due to the Kerr lensing (section 2.2) the modes resonant with the cavity are self focused most strongly in the crystal and completely overlap with the most efficiently pumped region. Therefore they are amplified to a higher amount than less intense modes with a bigger beam size. With each crystal transition this phenomena becomes more and more pronounced until weaker modes completely die out and a mode locked spectrum remains [79].

The oscillator is designed with $15 \%$ leakage rate in each cycle, which is realized with a wedged 


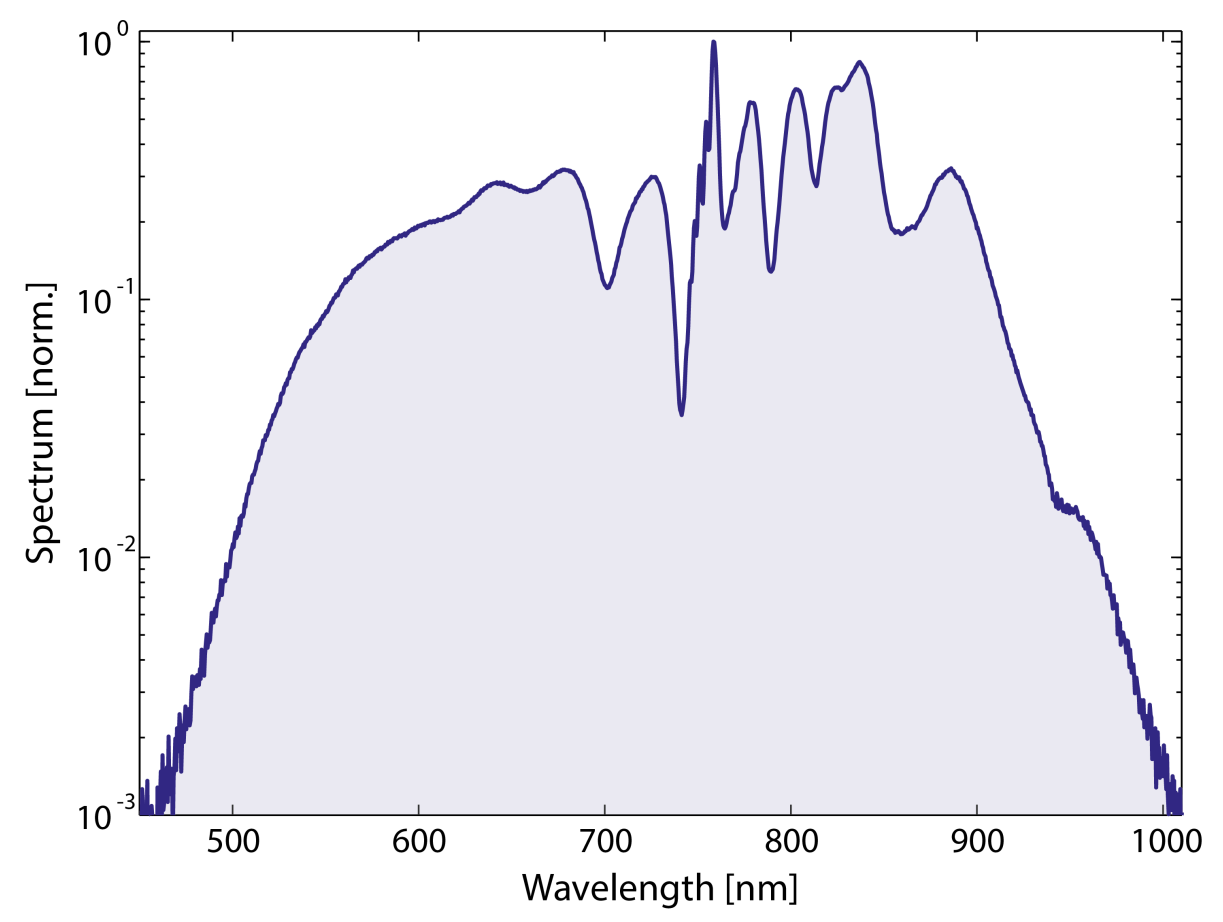

Figure 9.1: Spectrum of the few-cycle laser pulse.

output coupler. In order to compress the pulse temporally the beam is reflected two times off a pair of chirped mirrors after descending from the oscillator. For the performed experiments a few-cycle laser pulse with a stable and tunable CE phase (Fig.(19.14)) is necessary. In principle the dispersion introduced by the optical components in the beam path determines the CEP, however mechanical vibrations, air movement etc. decrease the CEP stability and change it from pulse to pulse. Thus after the chirped mirrors the beam is focused into a periodically poled lithium niobium crystal (PPLN) for difference frequency generation between the oscillator modes.

The temporal carrier envelope offset depicted in Fig.(9.14), corresponds to a frequency shift $f_{C E}$ in energy space, therefore the frequency comb of a mode locked laser $f_{f u n d}$ can be expressed as:

$$
f_{\text {fund }}=k \cdot f_{\text {rep }}+f_{C E} k=0,1,2, \ldots
$$

with the repetition rate $f_{\text {rep }}$. If the laser beam interacts with a nonlinear medium the difference frequency between laser modes $\mathrm{m}$ and $\mathrm{n}$ can be generated [80]:

$$
f_{\text {diff }}=\left(n \cdot f_{\text {rep }}+f_{C E}\right)-\left(m \cdot f_{\text {rep }}+f_{C E}\right)=(n-m) \cdot f_{\text {rep }} \quad n>m
$$

Additionally $f_{\text {fund }}$ and the originated $f_{\text {diff }}$ interfere in the nonlinear medium which 
produces a new frequency $f_{\text {beat }}[80]$ :

$$
f_{\text {beat }}=f_{\omega}-f_{\text {diff }}=\left(k \cdot f_{\text {rep }}+f_{C E}\right)-(n-m) \cdot f_{\text {rep }}=(k-n+m) \cdot f_{\text {rep }}+f_{C E}
$$

For $k-n+m=0$ the induced oscillation frequency is equal to the carrier envelope frequency $f_{\text {beat }}=f_{C E}$.

The described beating signal between the fundamental and the difference frequency component can be extracted with a low pass filter. It is passed on to a PI controller varying the pump power and therefore the nonlinear dispersion in the Ti:Sa crystal by adjusting the frequency of the acoustic-optical modulator. The PI controller stabilizes the emitted pulse train in such a way that every fourth pulse possesses an identical CEP. Therefore it is working at a quarter of the oscillator repetition rate frequency of $78 \mathrm{MHz}$. The high operating rate is the reason why this stage of the CEP stabilization mechanism is called the fast loop. To compensate the dispersion effects in the PPLN crystal the beam is subsequently reflected on another pair of negatively chirped multilayer mirrors (appendix 9.2). Subsequently the CEP stabilized laser pulses possess a duration of approximately $6 f s$ and a spectral width of $\lambda=600-1000 \mathrm{~nm}$. The average output power amounts $280 \mathrm{~mW}$ which corresponds to a pulse energy of approximately $4 \mathrm{~nJ}[80]$.

\section{Chirped Pulse Amplification}

In the amplifier stage the beam energy is increased by five orders of magnitude. To avoid damage of the optical components the pulse is stretched in time by introducing a chirp (section 9.5) to decrease the peak intensity (chirped pulse amplification [81]). After the oscillator a Faraday isolator turns the polarization of the laser field independent to the transmission direction. As it is encircled by two perpendicular polarization filters back reflection into the oscillator is prevented. Subsequently the pulse is stretched and positively chirped by transmission through a thick glass block. After the amplification the recompression is achieved with a prism compressor compensating the second order dispersion from the glass and the amplifier crystal [80]. The amplifier medium is again a Ti:Sa crystal, cut at Brewster's angle to minimize reflection losses and pumped by a Nd:YLF laser with pulses of nanosecond duration at a central wavelength of $\lambda=532 \mathrm{~nm}$. The beam is transmitted nine times through the gain medium. A mask with perforations of the size of the beam diameter additionally determines the beam path and prevents the 'cavity' from lasing. Due to the lack of higher repetition pump sources a pulse pick arrangement based on a Pockels cell encircled by two perpendicular polarizers only transmits a pulse approximately every $330 \mu s$. Thus the laser repetition rate is reduced to $3 \mathrm{kHz}$. As the pick frequency is adjusted to the carrier envelope frequency $f_{C E}$ only pulses with identical CEP are amplified. Additionally unintended amplified spontaneous emission is filtered out [80].

As the spectrum of the seed is broader than the energetic amplification bandwidth of Ti:Sa crystal, not all modes are supported. This gain narrowing decreases the spectral width of the amplified pulse and therefore increases its temporal transform-limit (for the Gaussian pulse 
$\left.\Delta v \cdot \Delta \tau=\frac{2 \ln (2)}{\pi}\right)$ to approximately $23 f$ s. The pulse is compressed to this minimal duration by a combination of 4 glass prisms and a set of chirped mirrors. If only prisms were used the high peak intensity in the last prism would cause undesired nonlinear effects. After the amplifier the pulse energy amounts $1.1 \mathrm{~mJ}$, with a pulse duration of $23 \mathrm{fs}$ at a repetition rate of $3 \mathrm{kHz}$ [78].

\section{Hollow Core Fiber and Multilayer Mirrors}

In order to generate few-cycle pulses, the spectral bandwidth has to be broadened to more than one octave. Therefore the beam is focused into a $1 \mathrm{~m}$ long hollow core fiber with a core diameter of $260 \mu \mathrm{m}$. The fiber is filled with $1.8 \mathrm{bar}$ Neon gas in which additional frequency components are established on both sides of the spectrum by self phase modulation (chapter 2.1). After the spectral broadening in the fiber a wavelength range from $\lambda=400-1000 \mathrm{~nm}$ is covered (Fig.(9.1)). As the energy transmission of the fiber amounts approximately $50 \%$ a pulse energy of approximately $500 \mu \mathrm{J}$ remains [82]. The temporal compression is performed subsequently by three pairs of low loss negatively chirped multilayer mirrors (Fig. 9.16 B)) designed to compensate the positive material dispersion the pulse accumulates during propagation through glass and air on its way to the experiment (appendix 9.2). Thus the pulse duration is decreased bellow 4 s (Fig.(6.4)). On the way through the amplifier and the fiber the influence of the different optical components and thermal instabilities require an additional CE phase stabilization. As these drifts evolve slowly [78], it is sufficient to operate on a $100 \mathrm{~Hz}$ timescale and therefore is called the slow loop. After the fiber and the chirped mirrors the spectrum is broad enough to perform a f-2f CE phase measurement. Therefore about $4 \%$ of the light are focused into a BBO crystal by a parabolic mirror to generate the second harmonic $f_{2 \omega}$ of the fundamental frequency $f_{\omega}$. A short pass filter isolates the second harmonic $f_{2 \omega}$ and the high energy part of the fundamental spectrum. Additionally the polarization of the second harmonic is matched to the fundamental by turning it by $90 \circ$ and the intensities of both beams are adapted. Subsequently the spectral interference $f_{\text {beat }}$ of the fundamental $f_{\omega}$ and frequency doubled light $f_{2 \omega}$ is recorded in a spectrometer and Fourier transformed by a software code. As the second harmonic $f_{2 \omega}=2 \mathrm{~m} \cdot f_{\text {rep }}+f_{C E}$ contains information about the carrier envelope frequency $f_{C E}$ the beating frequency $f_{\text {beat }}$ between $f_{2 \omega}$ and $f_{\omega}$ corresponds to $f_{C E}[83]$

$$
f_{\text {beat }}=2 f-f_{2 \omega}=2 n \cdot f_{\text {rep }}+2 \cdot f_{C E}-2 n \cdot f_{\text {rep }}-f_{C E}=f_{C E}
$$

To adjust the CEP one of the prism in the amplifier compressor is moved on a $\mu \mathrm{m}$ scale to adapt the amount of glass in the beam path. The changes in dispersion caused by these small movements are negligible as they have no influence on the pulse duration or beam pointing, but alter the CEP in the desired way. 


\subsubsection{Laser System Emitting Few-Cycle Pulses with $1 \mathrm{~mJ}$ Pulse Energy}

The laser system employed for the APS experiment in the gaseous phase (section 6.3) and the waveform synthesizer (section 7.1) is constructed very similarly to the arrangement described in the previous chapter (appendix 9.1): it is composed of an oscillator, two chirped pulse amplification stages, a hollow core fiber and negatively chirped dielectric multilayer mirrors for pulse compression (appendix 9.2). Thus sub 4fs pulses (Fig.(6.5)) with an energy of $1 \mathrm{~mJ}$ and a spectral bandwidth extending from $400 \mathrm{~nm}$ to $1050 \mathrm{~nm}$ centered at $780 \mathrm{~nm}$ (Fig.(9.23) supporting a Fourier limited pulse duration of $3 f s$ at a repetition rate of $4 \mathrm{kHz}$ are emitted [84].

The oscillator is build in the same modular concept as in the laser system presented in section 9.1.1 and generates pulses with a duration of $7 f s$ and an energy of $2 n J$ CEP stable at $1 / 4$ of the repetition rate. These pulses accumulate positive dispersion by propagation through a thick fused silica block and are thus stretched for the amplification by 9 transmissions through a pumped Ti:Sa crystal identical to the CPA scheme presented in section 9.1.1. The only difference is that the Pockels cell reduces the repetition rate to $4 \mathrm{kHz}$ instead of $3 \mathrm{kHz}$ and additionally a Dazzler [85, 86] compensates higher order phase distortions the pulse accumulates during amplification. Therefore the pulse energy is intensified to $1.1 \mathrm{~mJ}$ and a spectral bandwidth of $65 \mathrm{~nm}$. To achieve even higher field amplitudes the radiation is additionally boosted in a second amplification stage where it is transmitted three times through a cryo cooled $(70 \mathrm{~K})$ Ti:Sa crystal cut in Brewster's angle to minimize reflection losses pumped by a frequency doubled Nd:YLF laser with an output power of $50 \mathrm{~W}$ centered at $\lambda=532 \mathrm{~nm}$. Thus the pulse energy can be increased to $3 \mathrm{~mJ}$. The recompression of the stretched pulses and compensation of the positive material dispersion introduced by the optical components and the passage through air is performed with a transmission grating compressor [87]. As additional higher order phase distortions can be precisely compensated with the Dazzler the pulse duration is decreased close to the Fourier limit of $\tau_{F W H M} \approx 23 \mathrm{fs}$. Subsequently identical to the concept described in chapter 9.1.1 the pulse spectrum is broadened via self phase modulation (chapter 2.1) by transmission through a $1.15 \mathrm{~m}$ hollow core fiber filled with neon gas with an inner diameter of $380 \mu \mathrm{m}$. Thus a bandwidth extending from $40 \mathrm{~nm}$ to $1050 \mathrm{~nm}$ (centered at $780 \mathrm{~nm}$ (Fig.(9.23))) and a pulse energy of $1 \mathrm{~mJ}$ can be achieved at a repetition rate of $4 \mathrm{kHz}$. The CE phase is stabilized according to the scheme presented in the previous section with a fast and a slow loop (appendix 9.1.1).

\subsection{Chirped Dielectric Multilayer Mirrors}

The reflection of electromagnetic radiation off a periodic crystal is described by Bragg's law (Fig. (9.2) [38]. The external field excites dipoles in the material which emit spherical waves. The waves from the individual emitters in the different crystal layers interfere and for special angles of incidence $\alpha$ the difference in optical path length $\delta$ constitutes a multiple $m$ of the wavelength $\lambda$ resulting in constructive interference (Fig. (9.2)). 


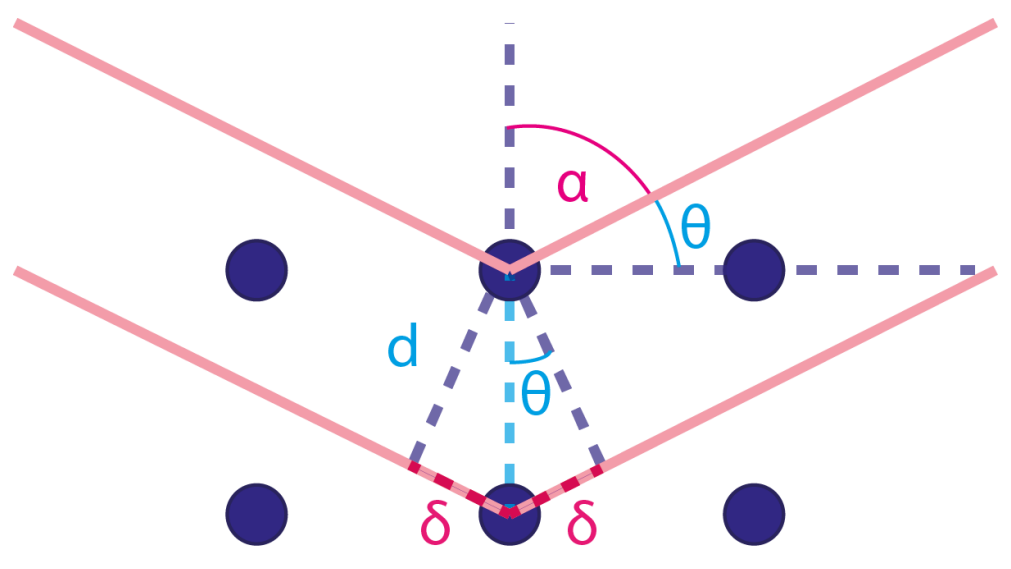

Figure 9.2: If Bragg's condition is fulfilled (Eqn. (9.6)) and the difference in path length $2 \delta$ between two beams diffracted at a periodic crystal structure amounts a multiple of the wavelength $m \lambda$, the two waves interfere constructively.

$$
2 \delta=m \lambda
$$

As the difference in optical path length $\delta$ is geometrically related to the lattice period $d$ by $\sin (\theta)=\frac{\delta}{d}$ with the Bragg angle $\theta=\pi-\alpha$ the Bragg condition for constructive interference is given by

$$
2 d \sin \theta=m \lambda
$$

Dielectric mirrors with a reflectivity of up to $99.9 \%$ for a very narrow wavelength range make use of this principle (Fig. (9.3) A). Multiple thin layers of different dielectric materials with refractive index $n_{1}$ and $n_{2}$ and a thickness $d_{1}$ and $d_{2}$ are combined with an optical path length $n_{1} \cdot d_{1}=n_{2} \cdot d_{2}=\lambda / 4$. As the radiation travels back and forth through the material and experiences a $\pi$ phase shift for reflection under $\theta=90^{\circ} n_{1} \cdot d_{1}=n_{2} \cdot d_{2}=\lambda / 4$ fulfills Eqn.(9.6) and thus results in almost perfect reflectivity. Even though the reflectivity at one interface is low the constructive interference between the beams reflected from the individual interfaces strongly increases the intensity. Bragg mirrors are commonly used in laser cavities as they can be optimized for transparency of the pump light and simultaneously high reflectivity at the laser wavelength. 


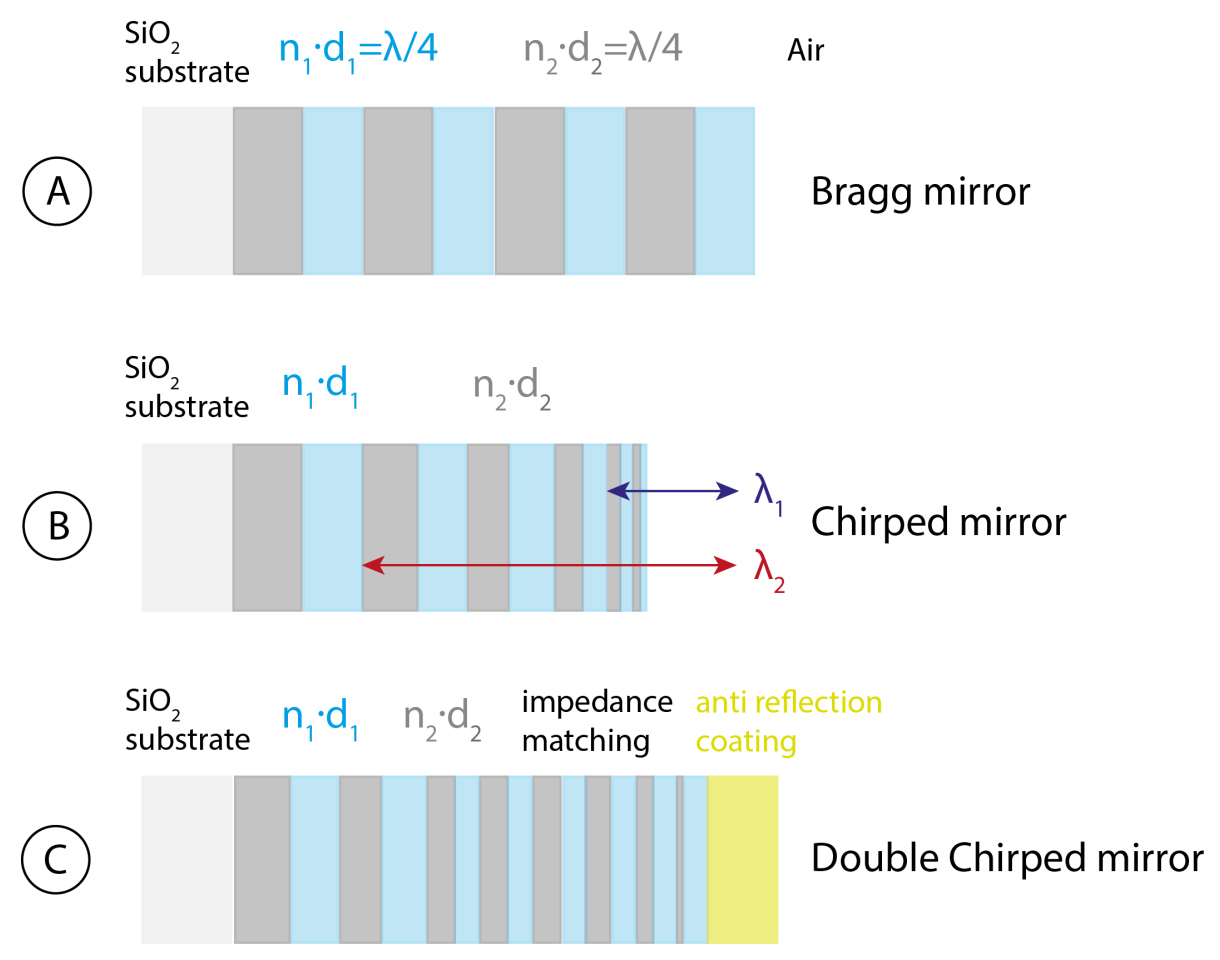

Figure 9.3: Schematic structure of Bragg mirrors (Panel A), chirped mirrors (Panel B) and double chirped mirror (Panel C) [88, 89].

In order to reflect a larger bandwidth the Bragg wavelength inside the multilayer structure has to be varied by chirping the optical path length $n_{1} \cdot d_{1}$ and $n_{2} \cdot d_{2}$ which is most easily realized by modification of the layer thickness. Thus different frequencies penetrate to a varying extent into the material and are reflected from different depth. This can be used to alter the temporal structure of a laser pulse and control its dispersion properties. Commonly negatively chirped dielectric multilayer mirrors are designed to compensate the positive material dispersion a pulse accumulates by propagation through glass and air. The layer structure is optimized for longer wavelength components to penetrate more deeply into the material than short ones and therefore travel a longer path than the high frequencies (Fig. 9.3 ) B) [89].

Despite a simple design of 2 different dielectrics stacked in multilayers with varying thickness introduces large oscillations in the group delay dispersion (section 9.5) due to two different effects: On the one hand the intersection from air to the material and the first dielectric interface form a Gire-Tournois interferometer with the air matter transition appearing as a semitransparent mirror with low reflectivity and the back surface as a perfect reflector. This creates a resonator which periodically modifies the spectral phase of the different frequency components. For wavelength resonant or antiresonant with the cavity no group delay dispersion is added, in between it oscillates between positive and negative values. This beating can be suppressed by addition of an anti reflection coating on top of the chirped Bragg system (Fig. (9.3) C) [88]. 
On the other hand the electromagnetic wave traveling back and forth in the chirped mirror can be described with a formalism common in microwave engineering by allocating an impedance to every layer pair. Fast changes of the impedance at the transition from air to matter causes big phase oscillations which can be suppressed by slow variation of the properties of the electrical system. This can be realized by chirping the thickness of the high refractive index material to slowly reach the desired extent and simultaneously varying the Bragg wavelength. Therefore this design approach is called the double chirped mirror (Fig. (9.3) C) [88].

For bandwidth as broad as one octave proprietary to few-cycle pulses a double chirped system is not sufficient to suppress the oscillations in the group delay dispersion resulting in residual higher order phase distortions. This challenge can be solved by coherent subtraction of the GDD oscillation after reflection off a pair of chirped dielectric multilayer mirrors designed to compensate the phase distortions of each other [90]. This approach is utilized to achieve the design displayed in Fig.(9.16 B).

\subsection{Generation of Isolated Attosecond Pulses}

The perturbative description of the nonlinear light-matter interaction (section 2) is valid as long as the amplitude of the external electric field is considerably lower than the internal atomic forces $E_{a t}$ and therefore the Keldysh parameter $\gamma>1$ (Eqn. 6.17)). The attracting electric field strength within an atom can be estimated to

$$
E_{a t}=\frac{e}{4 \pi \epsilon_{0} a_{0}^{2}}=5.14 \cdot 10^{11} \frac{\mathrm{V}}{\mathrm{m}}
$$

with the electron charge $e=1.6 \cdot 10^{-19} \mathrm{C}$, the electric permittivity of free space $\epsilon_{0}=$ $8.85 \cdot 10^{-12} \frac{F}{m}$ and the Bohr radius $a_{0}=5.29 \cdot 10^{-11} \mathrm{~m}$. If the amplitude of the radiation the material is exposed to exceeds $E_{a t}$ the Taylor expansion of the nonlinear polarization (Eqn.(2.4) does not converge anymore and new models have to be developed. 

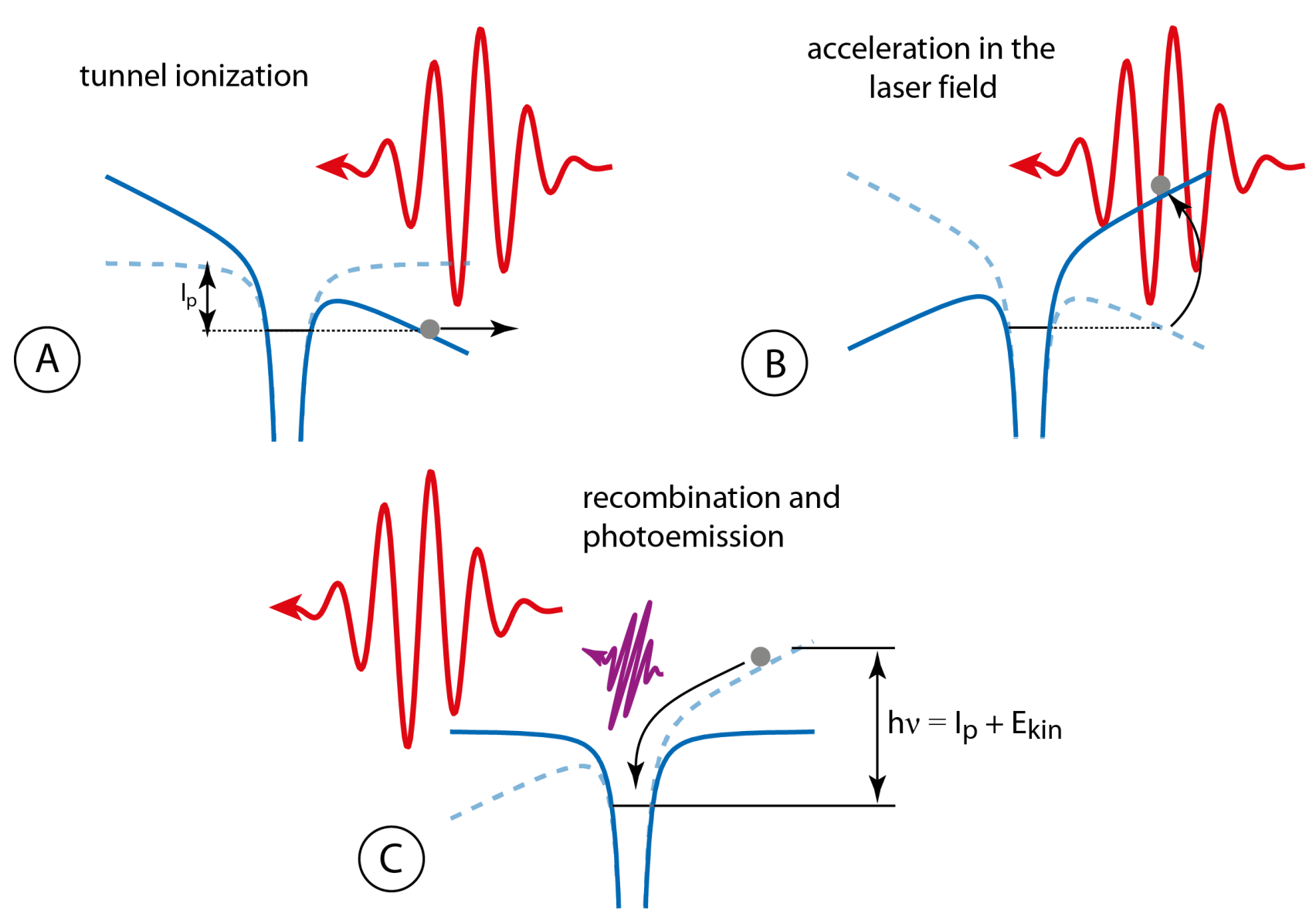

Figure 9.4: The 3 step model for high harmonic generation. Panel A: Under the influence of the external strong field electrons can tunnel through the Coulomb barrier of the atomic potential. Panel B: The freed electron is accelerated in the external field. Panel C: Under suitable conditions the electron recombines with the ion. Thereby a photon containing the kinetic energy accumulated in the external field $E_{k i n}$ together with the ionization potential $I_{p}$ is emitted [82].

The generation of high energetic radiation in the $X U V$ and soft $X$ ray range during the strongly nonlinear interaction of intense laser pulses $\left(I>10^{16} \frac{\mathrm{W}}{\mathrm{cm}^{2}}\right)$ with noble atoms in the gaseous phase is well described by the three step model [91,92], which combines quantum mechanical and classical considerations. A linearly polarized laser pulse with a field strength $E \geq E_{a t}$ deforms the atomic potential in such a way, that the electrons can tunnel through the Coulomb barrier into the continuum (Fig.(9.4) A). This process can be considered as an immediate reaction to the external electric field. After the charge escaped the attracting potential of the parent ion it can be treated as a classical free particle whose motion is determined by the driving force of the laser wave. The electron is freed around a peak of the external field and appears outside the Coulomb barrier without kinetic energy. Subsequently it is accelerated in the flank of the oscillating carrier field $E(t)=E_{0} \cos (\omega t)$. When the sign of $E(t)$ changes the electron is decelerated to zero velocity and changes direction in the second half of the subcycle. Thus it is accelerated back towards the mother ion and recombines close 
to the zero crossing of the electric field (Fig. (9.4) B). The gained kinetic energy the electron accumulated in the laser field $E_{k i n}$ together with the ionization potential $I_{p}$ are emitted as a photon coherent to the driving external light wave (Fig.(9.4) C). The trajectory of the electron $x(t)$ in the electric field $E(t)$ is calculated with the classical equation of motion [93]:

$$
F=m_{e} a-e E_{0} \cos (\omega t)=m_{e} \ddot{x}(t)
$$

with the free electron mass $m_{e}$ and charge $e$ and the acceleration $a$.

$$
\dot{x}(t)=-e \frac{E_{0}}{m_{e} \omega} \sin (\omega t)+x_{1} x(t)=e \frac{E_{0}}{m_{e} \omega^{2}} \cos (\omega t)+x_{1} t+x_{0}
$$

After tunneling the particle starts out at $x\left(t_{0}\right)=0$ with zero velocity $\dot{x}\left(t_{0}\right)=0$.

$$
\begin{gathered}
v(t)=e \frac{E_{0}}{m_{e} \omega}\left(\sin \left(\omega t_{0}\right)-\sin (\omega t)\right) \\
x(t)=e \frac{E_{0}}{m_{e} \omega^{2}}\left(\cos (\omega t)-\cos \left(\omega t_{0}\right)+\sin \left(\omega t_{0}\right)\left(\omega t-\omega t_{0}\right)\right)
\end{gathered}
$$

Therefore the kinetic energy of the electron amounts:

$$
E_{k i n}=\frac{1}{2} m_{e} v^{2}=\frac{e^{2} E_{0}^{2}}{2 m_{e} \omega^{2}}\left(\sin (\omega t)-\sin \left(\omega t_{0}\right)\right)^{2}=2 U_{p}\left(\sin (\omega t)-\sin \left(\omega t_{0}\right)\right)^{2}
$$

$U_{p}=\frac{e^{2} E_{0}^{2}}{4 m_{e} \omega^{2}}$ is the ponderomotive energy, which describes the average energy an electron gains when quivering in a cycle of the electric field. The electron and the ion can only recombine if $x\left(t_{r e c}\right)=0$ and thus the time of ionization $t_{0}$ and recombination $t_{r e c}$ are connected by:

$$
\cos \left(\omega t_{\text {rec }}\right)-\cos \left(\omega t_{0}\right)+\sin \left(\omega t_{0}\right)\left(\omega t_{\text {rec }}-\omega t_{0}\right)=0
$$

For one cycle of the laser pulse $\left(0<\omega t_{0}, \omega t_{\text {rec }}<2 \pi\right)$ Eqn.(9.13) can only be solved for $0<\omega t_{0}<\frac{\pi}{2}$. If the electron leaves the atoms later it cannot return to the ion and recombine.

According to Eqn. (9.12) $E_{k i n}\left(t_{r e c}\right)=E_{k i n}$ is given by

$$
\frac{E_{k i n}\left(t_{r e c}\right)}{U_{p}}=2\left(\sin \left(\omega t_{r e c}\right)-\sin \left(\omega t_{0}\right)\right)^{2}
$$

The associated phases for ionization $\omega t_{0}$ (blue curve) and recombination $\omega t_{\text {rec }}$ (red curve) connected by Eqn.(9.13) for each value of $E_{k i n}\left(t_{r e c}\right)$ (expressed in multiples of $U_{p}$ ) are schematically depicted in Fig.(9.5) [93].

Quantum mechanical calculation compute the maximum amount of kinetic energy gained by the electron during acceleration in the external electric field to $E_{k i n}^{\max }=3.17 U_{p}$ [94]. As 
the emitted photon contains this kinetic energy together with the ionization potential $I_{p}$, the cutoff of the harmonic spectrum amounts

$$
E_{\text {cutoff }}=I_{p}+3.17 U_{p}
$$

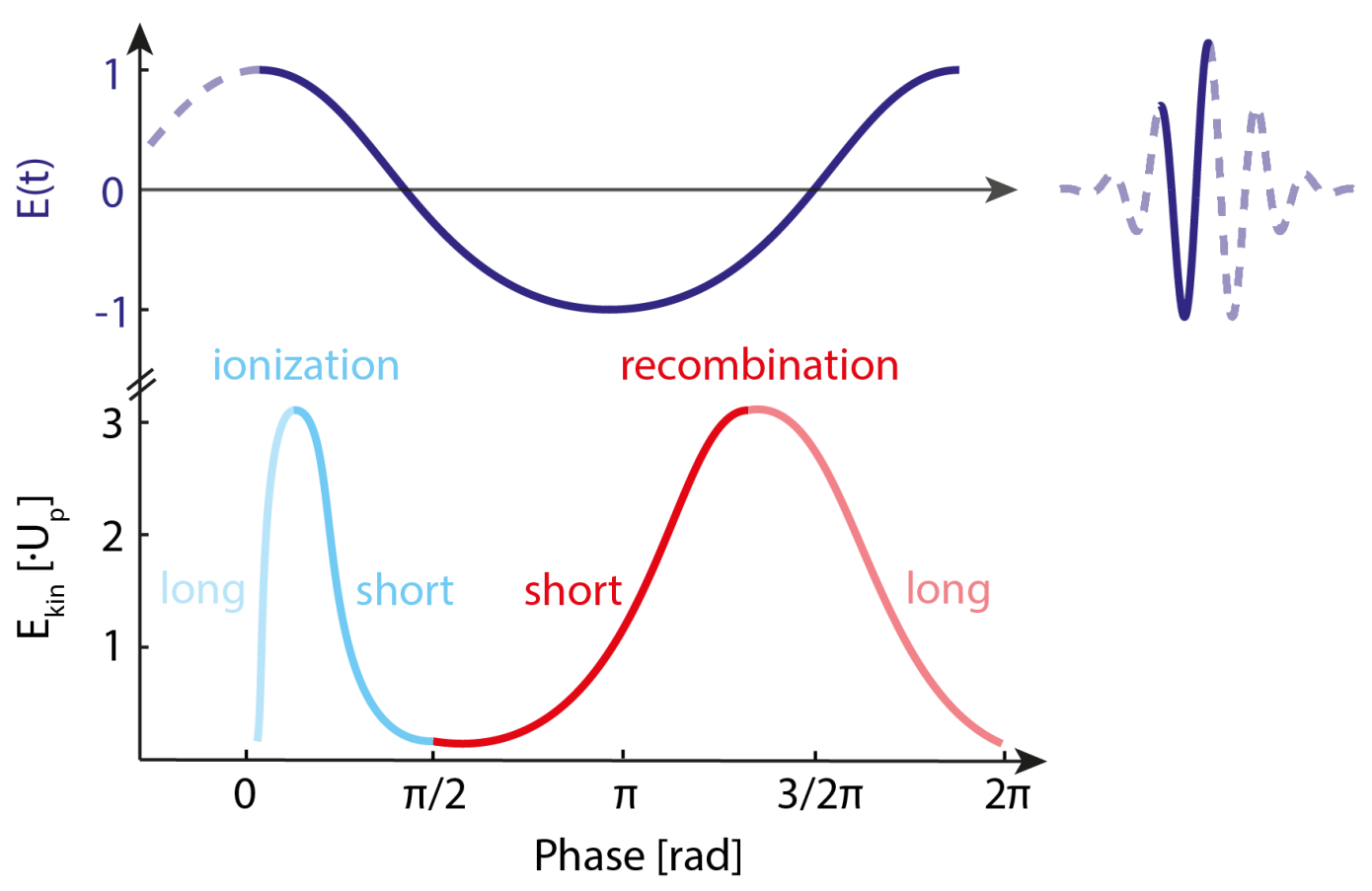

Figure 9.5: Eqn.(9.13) assigns phases of the external electric field $\omega t_{\text {rec }}$ (red line) when the electron and the parent ion recombine to the phase when the atom is ionized $\omega t_{0}$ (light blue line) resulting in a specific kinetic energy the particle gains during this period in the external field $E_{k i n}\left(t_{r e c}\right)=E_{k i n}$ (expressed in multiples of $\left.U_{p}\right)$. It is apparent that for $E_{\text {kin }}<E_{\text {kin }}^{\max }=3.17 U_{p}$ (Eqn. (9.15) ) always two pairs of $\omega t_{0}$ and $\omega t_{r e c}$ exist in one sub cycle (long and short trajectories) of the electric field (dark blue line). Even though on both paths electrons acquire the same amount of energy for the long trajectories ionization occurs earlier and recombination later than for the short ones. Thus the total period of time the electron spends under the influence of the electric field within one half cycle differs [93].

For photon energies below $E_{k i n}<E_{\text {kin }}^{\max }$ Eqn.(9.14) is solved by two pairs of phases $\omega t_{0}$ and $\omega t_{\text {rec }}$ (Fig. (9.5) short and long trajectories). Even though on both paths electrons acquire the same amount of energy for the long trajectories ionization occurs earlier and recombination later than for the short ones. Thus the total period of time the electron spends under the influence of the electric field within one subcycle differs [93].

As $E(t)=E_{0} \cos (\omega t)$ is a periodic function also multiples of the phase pairs $\omega t_{0}+m \pi$ and $\omega t_{r e c}+m \pi(m \in N)$ are valid solutions of Eqn.(9.13) and Eqn.(9.14). Thus for noble atoms in the gas phase which possess inversion symmetry electrons are freed close to every peak of the electric field and therefore XUV photons are generated near the zero crossing twice during every half cycle of the laser pulse (Fig.(9.6)). 
This means that the process occurs with twice the laser frequency $2 \omega$. The lowest spectral component in the Fourier transform of the high harmonic spectrum is the fundamental laser frequency $\omega$ itself, as all other contributions are separated by $2 \omega$, the spectrum only contains odd multiples of the original laser frequency.

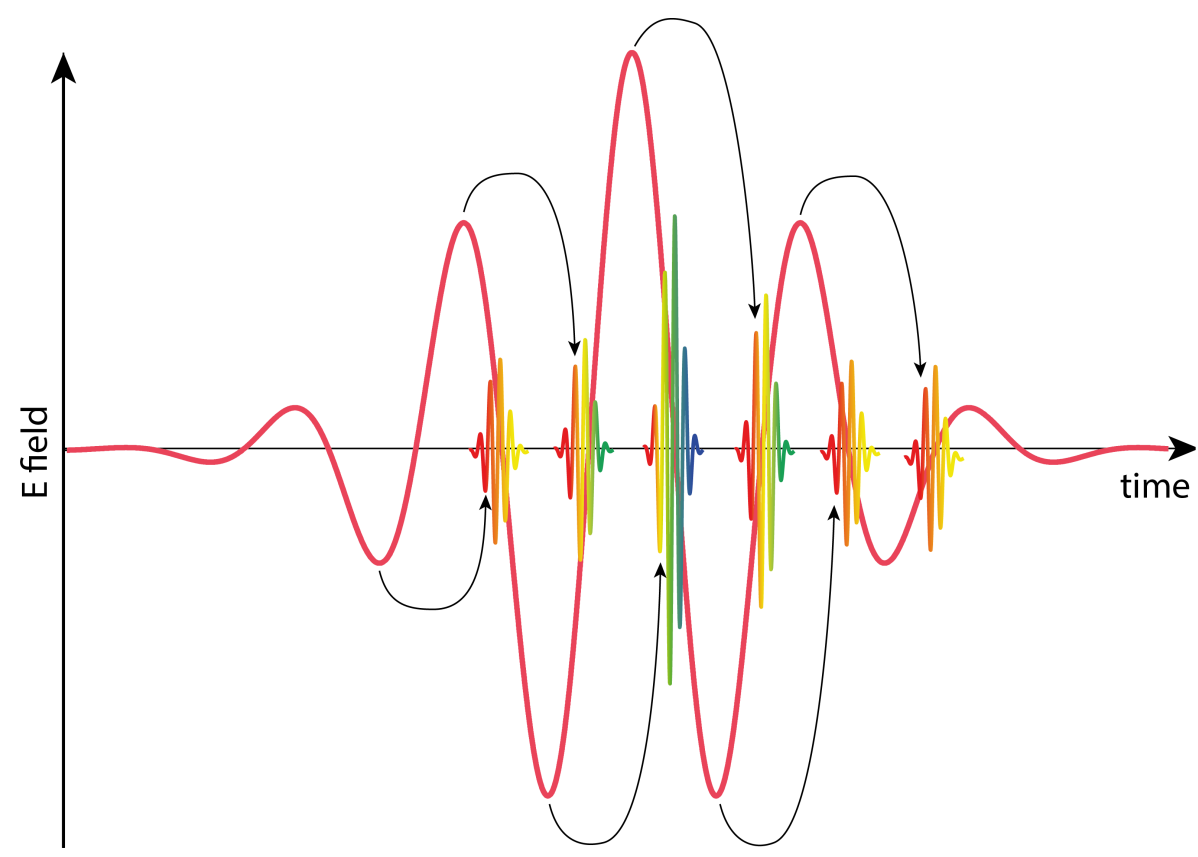

Figure 9.6: The emission of high harmonic radiation is temporally correlated to the driving laser pulse (red line). Electrons are freed close to the field maxima and accelerated in the flank of the electric wave until its sign changes. Then the particles are decelerated to zero velocity and finally fly in the opposite direction. Close to the zero crossing of the electric field the charges recombine with the parent ion and a short burst of high frequency light is emitted. The process happens twice in every sub cycle. The time period between ionization and recombination is indicated with black arrows. The higher the field strength at the time of ionization the more electrons are freed which is directly proportional to the amplitude of the emitted XUV pulse. The amount of energy the electrons accumulate in the electric field influences the frequency of the high harmonic radiation which is indicated in the color code of the emitted pulses: blue symbolizes the highest energy, red the lowest. As the time of birth and recombination and therefore the period in which the carriers are accelerated in the external field differs the harmonic light possesses an intrinsic positive chirp (section 9.5) [80]. 


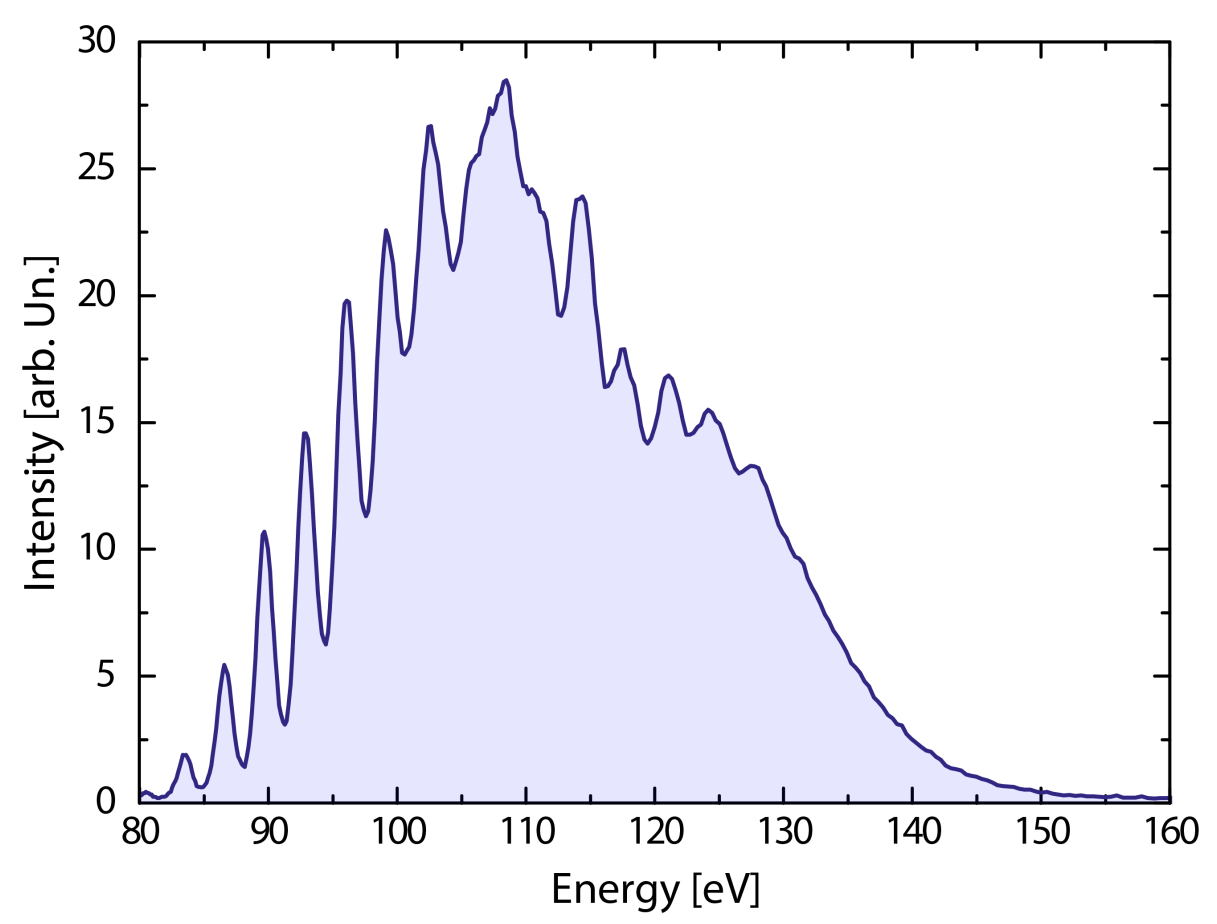

Figure 9.7: The high harmonic spectrum generated with a CEP stable cosine few femtosecond laser pulse (appendix 9.1.2) with an intensity $>1 \cdot 10^{16} \frac{\mathrm{W}}{\mathrm{cm}^{2}}$ in 140 mbar Neon gas after transmission through a $1.5 \mu \mathrm{m}$ thick Zirconium foil. The spectrum is modulated up to energies of $125 \mathrm{eV}$. In this frequency range XUV photons with the same energy are emitted several times throughout the pulse (Fig.(9.6)) which results in an interference structure in the spectrogram. The photons above $>125 \mathrm{eV}$ are generated only once in the most intense half cycle of the driving cosine field and form the non modulated cut off region.

In general the high harmonic spectrum generated with cosine carrier envelope phase stable few-cycle pulses can be divided into three parts according to the intensity of the emitted radiation: the first range contains low order harmonics and is well described by perturbation theory as the field amplitude strongly decreases with increasing order of the nonlinearity. Subsequently the modulated plateau region succeeds, in which the intensity remains almost constant independent of harmonic number as proof of the non convergence of the Taylor expansion (Eqn.(2.4) and thus the invalidity of the pertubative description for this energy range. It can be understood with the three step model described above. In the plateau region bursts of XUV light are emitted several times throughout the driving laser field. Thus radiation with the same energy appears at different instants in time and interferes visible as a fringe structure in frequency space (Fig.(9.6)). For cosine carrier envelope phase stable few-cycle pulses (Fig.(9.14)) the highest frequency photons are generated only once in the most intense half cycle of the driving laser field and form the non modulated cut off region. To isolate the single XUV pulses with durations of a few 100 as providing the attosecond time resolution in the streaking measurements this energy range is filtered out [93]. Fig.(9.7) depicts the high harmonic spectrum generated with a carrier envelope phase stable cosine sub 4 fs VIS/NIR laser pulse (Fig.(6.5)) with an intensity $>1 \cdot 10^{16} \frac{\mathrm{W}}{\mathrm{cm}^{2}}$ in $140 \mathrm{mbar}$ Neon gas 
after transmission through $1.5 \mu \mathrm{m}$ Zirconium. The spectrum is optimized to generate isolated attosecond pulses centered at $130 \mathrm{eV}$ for the APS experiment in the gaseous phase (section 6.3). Therefore the phase matching conditions are not fulfilled for low energy harmonics which interfere destructively during propagation in the Neon gas and therefore do not leave the medium they are generated in. This together with the transmission characteristics of the Zirconium filter and the limited spectral detection range of the spectrometer is the reason why the pertubative low harmonics are not visible and the intensity rises throughout the plateau region. Despite the vanishing fringe structure in the cutoff region where the most energetic XUV photons are only generated once in the central flank of the driving field is well pronounced.

\subsection{Attosecond Streaking Spectroscopy}

Not only the generation of isolated attosecond pulses is a challenging task, but also their temporal characterization. A sophisticated method which provides direct access to the electric field of the driving few-cycle pulse generating the high harmonic radiation (section 9.3 and the attosecond light burst itself, is the so called streaking technique [95]. Both pulses are focused with variable delay onto a dilute Neon gas jet. The XUV beam photo ionizes the atoms and the freed electrons are subsequently exposed to the influence of the VIS/NIR field. Depending on their time of birth $t_{0}$ they gain or loose part of their initial kinetic energy in the few-cycle field whose substructure therefore functions as a timing reference [55]. The photoelectron energy is determined by recording the time of flight the individual carriers require to reach a detector in direction of the laser polarization axis. In order to directly resolve the temporal evolution of the VIS/NIR electric field, the duration of the XUV pulse must be shorter than the examined field [95]. If the ionization potential $I_{p}$ of the atoms considerably exceeds the frequency of the VIS/IR photons and simultaneously is substantially smaller than the energy of the attosecond pulse $E_{X U V}$, the kinetic energy of the photoelectrons after escaping the atomic potential is given by [55]:

$$
E_{k i n}=\hbar \omega_{X U V}-I_{p}=\frac{1}{2} m v_{0}^{2}
$$

$v_{0}$ is the velocity the electrons possess directly after photo ionization at $t_{0}$ without any influence of the VIS/NIR laser field $E(t)$. In the classical picture the subsequent acceleration $a$ in the few-cycle field can be expressed with

$$
F=m a=-e E(t)
$$

$A(t)$ is the vector potential of $E(t)$.

$$
E(t)=-\frac{\partial A(t)}{\partial t}
$$

Therefore the velocity of the electron in the electric field is

$$
v(t)=-\frac{e}{m} \int_{t_{0}}^{t} E\left(t^{\prime}\right) d t^{\prime}=\frac{e}{m} \int_{t_{0}}^{t} \frac{\partial A\left(t^{\prime}\right)}{\partial t^{\prime}} d t^{\prime}=\frac{e}{m} A(t)-\frac{e}{m} A\left(t_{0}\right)+v_{0}
$$


The time resolution of the streaking recorder is determined by the temporal profile of the attosecond pulse. In frequency space the XUV radiation possesses a certain spectral bandwidth (Fig. (9.6)), which determines the energy distribution of the freed photoelectron. The measurement of this energy distribution is additionally effected by the temporal pulse structure as the time the electrons require to reach the detector is recorded representatively. Particles set free by photons arriving later in the XUV pulse seem to have lower kinetic energy than photo electrons which are born at the pulse front.

The additional influence of the VIS/NIR field makes these carriers distinguishable, as the alteration of the energy of the electrons by the few-cycle pulse depends on their time of birth $t_{0}$ (Eqn.(9.19)). Therefore comparing the static and streaked photoelectron spectra gives insight into the temporal properties of the XUV pulse. Fig. 9.8 A dashed dark blue line) depicts a simulated photoelectron spectrum [55] generated with a Fourier limited attosecond XUV pulse. The red line displays the prediction of the quantum mechanical model for the spectral modifications caused by the interaction with a few-cycle laser pulse when the attosecond pulse overlaps with a zero crossing of the rising flank of the vector potential $A(t)$ (Fig 9.9p).The energy distribution is broadened (Fig. (9.8 A red line)) as the photoelectrons emitted at the beginning of the XUV pulse are partly decelerated in the negative part of the vector potential while the electrons born at the end of the pulse are immediately accelerated by the VIS/NIR influence (Eqn. (9.19]) [55].

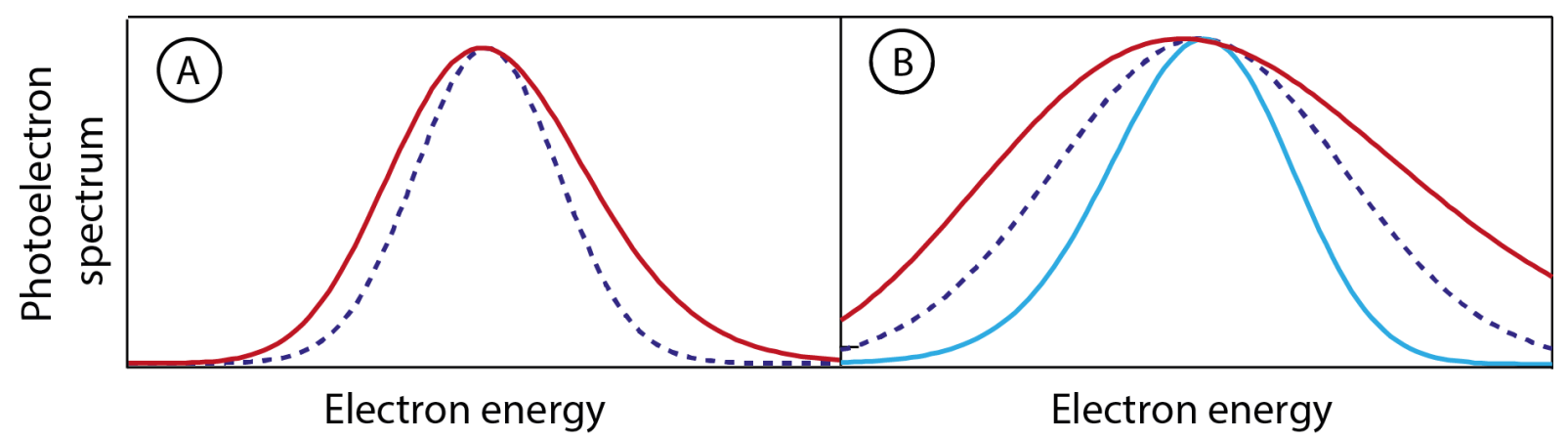

Figure 9.8: Panel A: The photoelectron spectrum generated by a transform limited XUV pulse (dashed dark blue line). It is modified in the presence of an optical strong field. If the attosecond pulse overlaps with a zero crossing of the rising flank of the vector potential $A(t)$ (Fig.9.9p) the energy distribution is broadened (red line). Panel B: The photoelectron spectrum generated by a chirped XUV pulse in the absence of a strong field (dashed dark blue line). If a positively chirped attosecond pulse overlaps with a zero crossing of the rising flank of the strong field the photoelectron spectrum is broadened (red curve). For a XUV pulse with negative chirp the influence of the strong field narrows the bandwidth of the photoelectron distribution (light blue line). [55] 


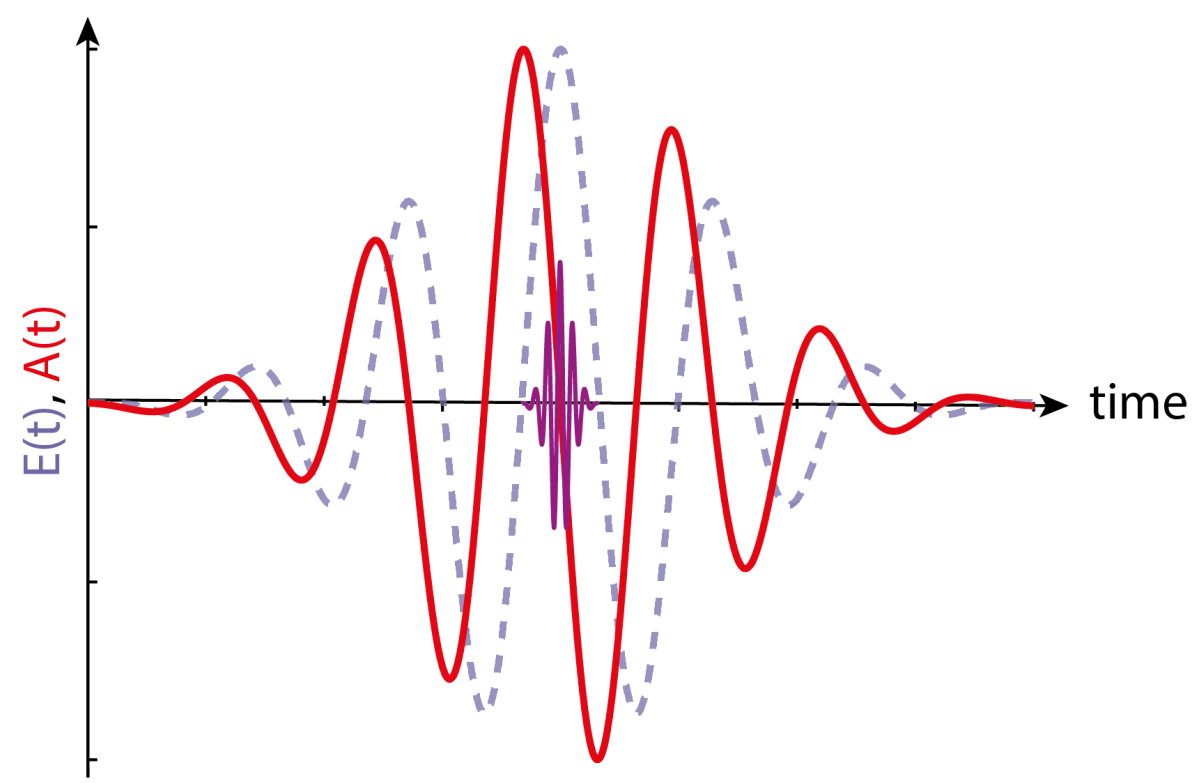

Figure 9.9: The ultrashort attosecond pulse (purple line) overlaps with a maximum of the electric field $E(t)$ of an optical few-cycle pulse (blue dashed line). This corresponds to a zero crossing of the rising flank of its vector potential $A(t)$ (red line)

By varying the delay between XUV and VIS/NIR pulse, the entire few-cycle field can be sampled (Fig.(6.5)). The recorded spectrogram provides direct access to the temporal profile of the vector potential of the examined field $A(t)$ (Eqn. (9.19) ) and therefore to the complete amplitude and phase information of the VIS/NIR field. With an algorithm disentangling the autocorrelation trace the temporal profile of the attosecond pulse can be extracted [55], [96].
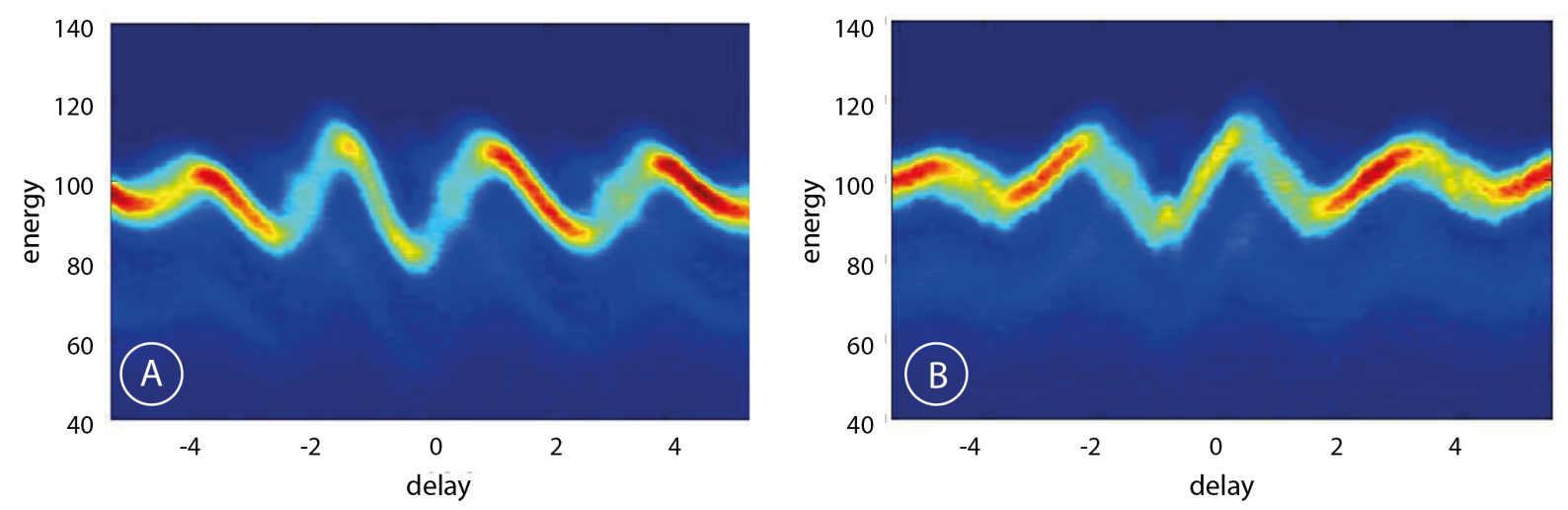

Figure 9.10: The streaking spectrogram recorded with a XUV pulse wit positive (Panel A) and negative chirp (Panel B) [97].

A chirp of the attosecond pulse possesses a characteristic signature imprinted on the photoelectron spectrum modified by the influence of the VIS/NIR pulse. Fig. (9.9 B) depicts the energy distribution for the delay step when the XUV radiation overlaps with a maximum 
of the electric field and therefore a zero crossing of the rising flank of the VIS/NIR vector potential $A(t)$.

If the chirp is positive low frequency components arrive earlier than high energetic photons and are partially decelerated by the negative $A(t)<0$ (Eqn. (9.19)), while the high frequency components are further accelerated under the influence of the positive $\vec{A}(t)>0$. Therefore the photoelectron spectrum is broadened (Fig $9.8 \mathrm{~B}$ red line) compared to the field free case (Fig.9.8 B dashed dark blue line). For a XUV pulse with negative chirp the high frequencies temporally precede the long wavelength and are decelerated more strongly than the delayed low energy part which decreases the bandwidth of the photoelectron distribution (Fig 9.8 B light blue line) [55].

Accordingly a positively chirped photoelectron distribution is narrowed if it overlaps with the falling flank of the VIS/NIR vector potential while a negative chirp broadens the spectrum. This effect is well visible in the streaking traces recorded with chirped attosecond pulses depicted in Fig. (9.10) [80], [82]. The XUV radiation is reflected of metal multilayer mirrors (section 9.2) particularly designed for altering penetration depth of different frequency components [97], [98]. As the streaking trace is directly proportional to the vector potential $A(t)$ of the VIS/NIR field (Eqn. (9.19)) the bandwidth of the spectrogram recorded with a positively chirped attosecond pulse is narrowed in the falling flanks and broadened when $\frac{\partial A(t)}{\partial t}>0$ (Fig. 9.10 A). If the XUV possesses a negative chirp the energy spread of the photoelectrons is increased for $\frac{\partial A(t)}{\partial t}<0$ and decreased in the rising flank of $A(t)$ (Fig. (9.10) B).
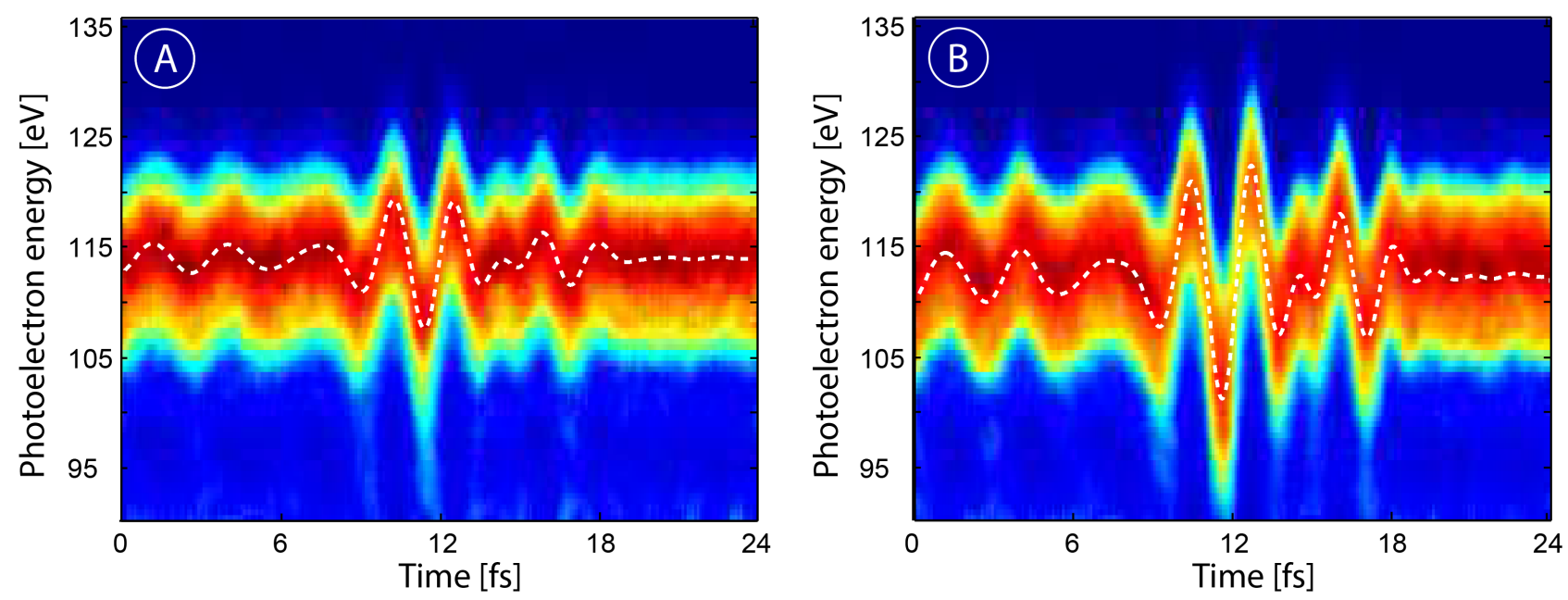

Figure 9.11: The streaking spectrogram of the reference pulse (Panel A) and the strong field (Panel B) recorded for the APS measurement in $10 \mu \mathrm{m}$ fused silica. The center of mass of the photoelectron distribution (white dashed line) is proportional to the vector potential of the field.

The streaking spectrogram recorded for the APS experiment in fused silica are depicted in Fig. (9.11) (section 6.4.1). Panel A shows the trace of the weak reference field which is transmitted through the sample with an amplitude of $E_{r e f}=(5.9 \pm 0.1) \cdot 10^{9} \frac{\mathrm{V}}{\mathrm{m}}$. In Panel B 
the spectrogram of the strong field which possesses a field strength of $E=(2.6 \pm 0.1) \cdot 10^{10} \frac{\mathrm{V}}{\mathrm{m}}$ at the sample is displayed.

\subsection{Frequency Resolved Optical Gating}

The TG FROG (transient grating frequency resolved optical gating) technique is based on a third order auto-correlation where the polarization wave is generated in a four wave process at the fundamental frequency. The input pulse is divided into three identical replica. Two of them overlap in a Kerr medium and their sinusoidal intensity pattern nonlinearly modifies the optical material density. Their delay $\tau$ with respect to the third probe pulse $E(t)$ is varied. In temporal overlap $E(t)$ is diffracted off the induced refractive index grating. Hence $|E(t-\tau)|^{2}$ can be considered as a gate function $g(t-\tau)$ for the investigated electric field. For each delay step the generated polarization wave is spectrally resolved. As the FROG measurement is mainly performed to extract the pulse shape constants are ignored in the following and the polarization is replaced by the signal field $E_{\text {sig }}(t, \tau)=|E(t-\tau)|^{2} E(t)$. By varying $\tau$ the spectrogram $I^{T G}(\omega, \tau)$ can be recorded [74].

$$
\begin{gathered}
I^{T G}(\omega, \tau)=\left|\int_{-\infty}^{\infty} E_{\text {sig }}(t, \tau) \exp (-i \omega t) d t\right|^{2}=\left|\int_{-\infty}^{\infty} E(t) g(t-\tau) \exp (-i \omega t) d t\right|^{2} \\
I^{T G}(\omega, \tau)=\left.\left.\left|\int_{-\infty}^{\infty}\right| E(t-\tau)\right|^{2} E(t) \exp (-i \omega t) d t\right|^{2}
\end{gathered}
$$

From $I^{T G}(\omega, \tau)$ the signal field can be numerically unambiguously reconstructed in frequency space $\widetilde{E}_{\text {sig }}(t, \Omega)[74]$.

$$
\begin{gathered}
I^{T G}(\omega, \tau)=\left|\int_{-\infty}^{\infty} \int_{-\infty}^{\infty} \widetilde{E}_{\text {sig }}(t, \Omega) \exp (-i \Omega \tau) \exp (-i \omega t) d t d \Omega\right|^{2} \\
E_{\text {sig }}(t, \tau)=\int_{-\infty}^{\infty} \widetilde{E}_{\text {sig }}(t, \Omega) \exp (-i \Omega \tau) d \Omega
\end{gathered}
$$

From $E_{\text {sig }}(t, \tau)$ the electric field $E(t)$ is accessible.

The schematic setup of the TG FROG is depicted in Fig.(9.12). To fulfill the phase matching condition for the four wave process and spatially separate the generated signal the folded BOXCARS arrangement is employed [99]: the incoming beam is transmitted through a mask with 3 perforations positioned in the corners of a square. They divide the pulse into the required 3 replica of the input field. Two of them hit the same half of a double mirror which focuses them onto a $100 \mu m$ thick fused silica plate. The third probe beam is reflected from the movable double mirror side and variably delayed. In temporal overlap the generated polarization wave appears in the forth corner of the square formed by the pump and probe beams (Fig. (9.12) [99]. Its spectrum is recorded for each delay step. 


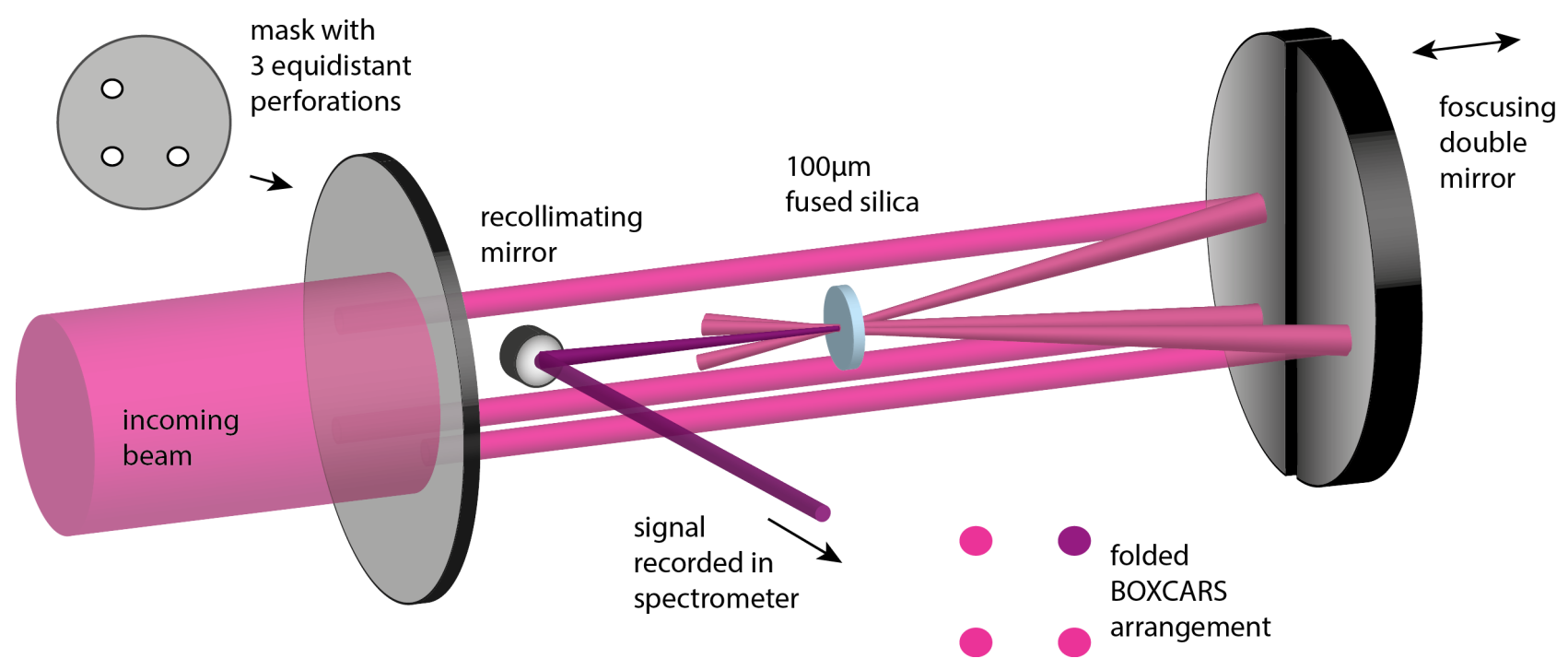

Figure 9.12: Schematic setup of the TG FROG with the folded BOXCARS arrangement [99].

The phase of an electric field can be expressed in time $\phi(t)$ or frequency space $\varphi(\omega)$.

$$
\begin{gathered}
E(t)=E_{0} \exp (i \omega t) \exp (-i \phi(t))+c . c . \\
\widetilde{E}(\omega)=\widetilde{E}_{0} \exp (-i \varphi(\omega))+\text { c.c. }
\end{gathered}
$$

with

$$
\widetilde{E}(\omega)=\frac{1}{2 \pi} \int_{-\infty}^{\infty} E(t) \exp (-i \omega t) d t
$$

For convenient analytical handling the spectral phase, $\varphi(\omega)$, can be developed into a Taylor expansion around the central frequency $\omega_{0}$.

$$
\varphi(\omega)=\varphi_{0}+\left(\omega-\omega_{0}\right) \varphi_{1}+\frac{1}{2}\left(\omega-\omega_{0}\right)^{2} \varphi_{2}+\frac{1}{6}\left(\omega-\omega_{0}\right)^{3} \varphi_{3}+\ldots
$$



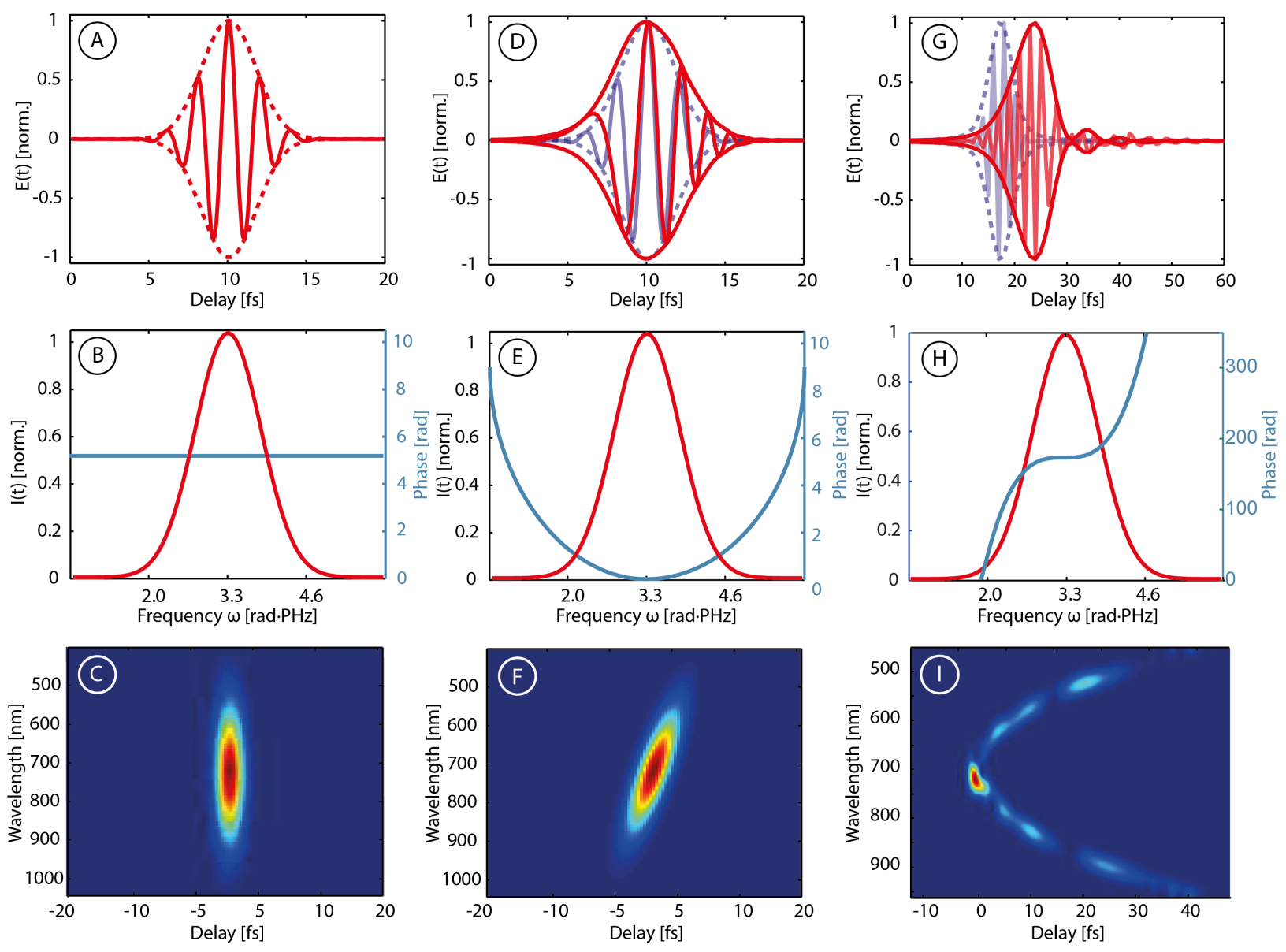

Figure 9.13: Theoretical simulation of the impact of higher order contributions to the spectral phase: A transform limited Gaussian pulse (Panel A) possesses a constant spectral phase (Panel B: phase blue line, intensity envelope red line). In the corresponding TG FROG spectrogram all spectral components are centered at temporal overlap (Panel C). A positive quadratic phase contribution (Panel E: phase blue line, intensity envelope red line) chirps the pulse (Panel D: the chirped pulse (red line) is compared to the transform limited field (blue line)). As high frequency components are retarded more strongly than long wavelength the TG FROG spectrogram is tilted (Panel F). A positive cubic phase term (Panel H: phase blue line, intensity envelope red line) leads to post pulses (Panel G red line, the blue line represents the transform limited pulse) and manifests itself in a $C$ shape structure in the TG FROG trace (Panel I). 
A transform limited pulse possesses the shortest possible duration at a given spectral width. All wavelength interfere constructively at the same time (Fig. (9.13 A)). The phase of these pulses is independent of wavelength $\varphi(\omega)=\varphi_{0}=$ const. and invariant under Fourier transformation $\varphi(\omega)=\phi(t)=\varphi_{0}$ (Fig. (9.13 B)). As $\varphi_{0}$ determines the relation between the pulse envelope and the carrier field it is called the carrier envelope phase. Fig.(9.14) shows that $\varphi_{C E}$ determines the amplitude ratio between the field maxima throughout the pulse. A cosine pulse (blue line) possesses a global maximum whereas the absolute value of the maximum field amplitude is reached twice during a sine pulse (red line).

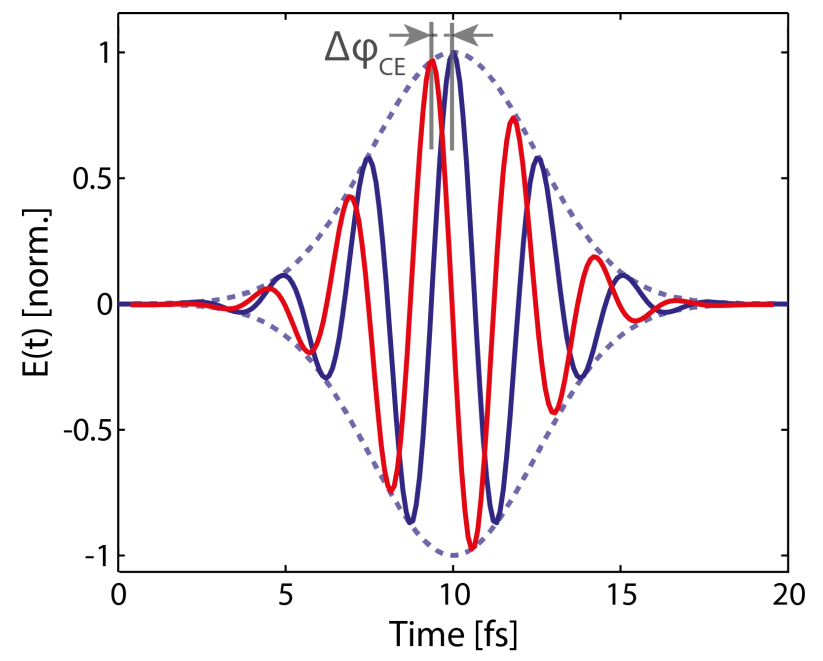

Figure 9.14: Two few-cycle pulses with different carrier envelope phase $\varphi_{C E}$ : the blue line displays a cosine, the red line a sine pulse.

The $\varphi_{C E}$ is measurable with attosecond streaking (appendix 9.4) but cannot be accessed with the FROG technique. A theoretical example of the TG FROG spectrogram of a bandwidth limited pulse is depicted in Fig.(9.13 C).

A non zero $\varphi_{1}$ in Eqn.(9.27) adds a linear frequency component to $\phi(\omega)$. This corresponds to a shift of the entire pulse in time. Except for interferometric applications the absolute arrival time of the pulse is usually irrelevant and therefore the linear frequency phase term can be ignored in general.

A quadratic phase contribution $\varphi_{2}$ in Eqn. (9.27) alters the instantaneous frequency of the pulse (Eqn.(2.24)) as it retards long and short wavelength to a different amount. This phenomena chirps the carrier wave and modifies the pulse duration (Fig.(9.13 D)). In the visible materials like glass or air introduce positive dispersion and accelerate low frequencies compared to high ones. The introduced chirp tilts the TG FROG spectrogram (Fig. (9.13 F)) as the long wavelength arrive earlier than the high frequency part [74].

Depending on its sign a cubic phase term $\varphi_{3}$ (Eqn.(9.27) retards the margins of the spectrum less or more strongly than the central part. This leads to the formation of pre- or post pulses (Fig. (9.13 G)). A cubic phase contribution is visible as a $C$ shaped structure in the TG FROG spectrogram (Fig. $9.13 \mathrm{I})$ ). 
The temporal structure of most pulses can be described under consideration of nonlinear phase terms up to third order (Eqn.(9.27)). Fig.(9.15) displays the TG FROG spectrogram of the few-cycle laser pulse which is used for the APS experiment in the gas phase (appendix 9.1.2).

The retrieved spectral intensity and phase are plotted in Fig.(9.16 A). The wavelength range between $500-1000 \mathrm{~nm}$ contains the main part of the pulse energy. In this region the spectral phase varies little with frequency. It merely exhibits a slight third order nonlinearity. The weak blue part of the spectrum below $<500 \mathrm{~nm}$ possesses a highly sophisticated phase structure. As described in appendix 9.1.2 the whole spectrum of the pulse is compressed with one set of chirped multilayer mirrors (appendix 9.2). The design is chosen as the best compromise between compensating the phase nonlinearities and maintaining the maximum reflectivity throughout the entire bandwidth. It is considered the best solution to ignore the complicated phase structure below $500 \mathrm{~nm}$ and optimize the design for the spectrum between $500-1000 \mathrm{~nm}$ which contains the main part of the pulse intensity. The reflectivity and group delay dispersion $G D D=\frac{\partial^{2} \varphi}{\partial \omega^{2}}$ of the employed chirped mirrors are depicted in Fig.(9.16 B). They are fabricated by the group of Dr. Volodymyr Pervak (LMU). The retrieved duration of the pulse is below $\tau_{F W H M}<4 f s$.

In order to verify the reliability of the TG FROG measurement it is tested whether the dispersion of $1 \mathrm{~mm}$ fused silica can be measured correctly. First the dispersion contribution is theoretically added to the group delay dispersion $G D D=\frac{\partial^{2} \varphi}{\partial \omega^{2}}$ of the measured pulse (Fig.(9.17) light blue line). The obtained result is compared to the GDD extracted from a TG FROG spectrogram recorded under identical conditions for the pulse after transmission through $1 \mathrm{~mm}$ additional glass. Fig.(9.17) displays the agreement between the theoretical prediction and the experimental result.
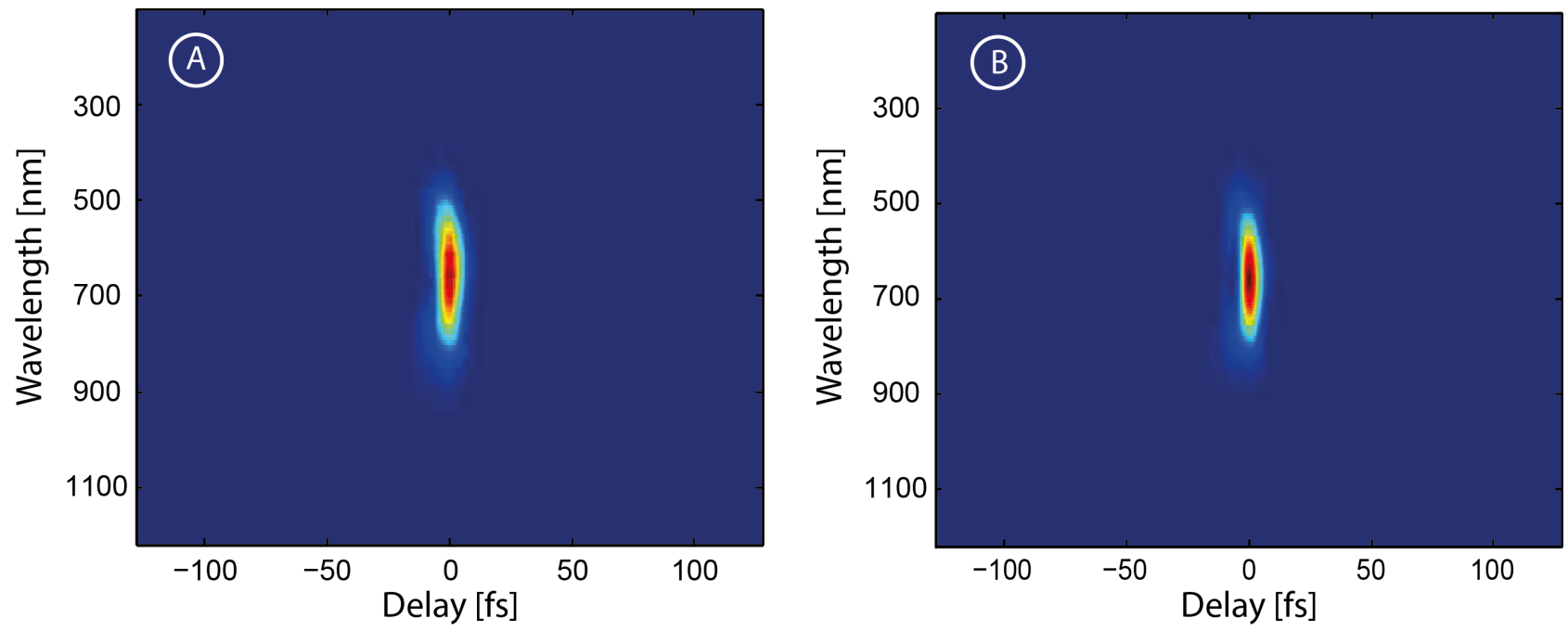

Figure 9.15: The recorded (Panel A) and reconstructed (Panel B) TG FROG spectrogram of the few-cycle field employed in the APS measurement in gas (appendix 9.1.2). The FROG error which measures the numerical derivation between the two traces is $5 \cdot 10^{-3}$ [74]. 

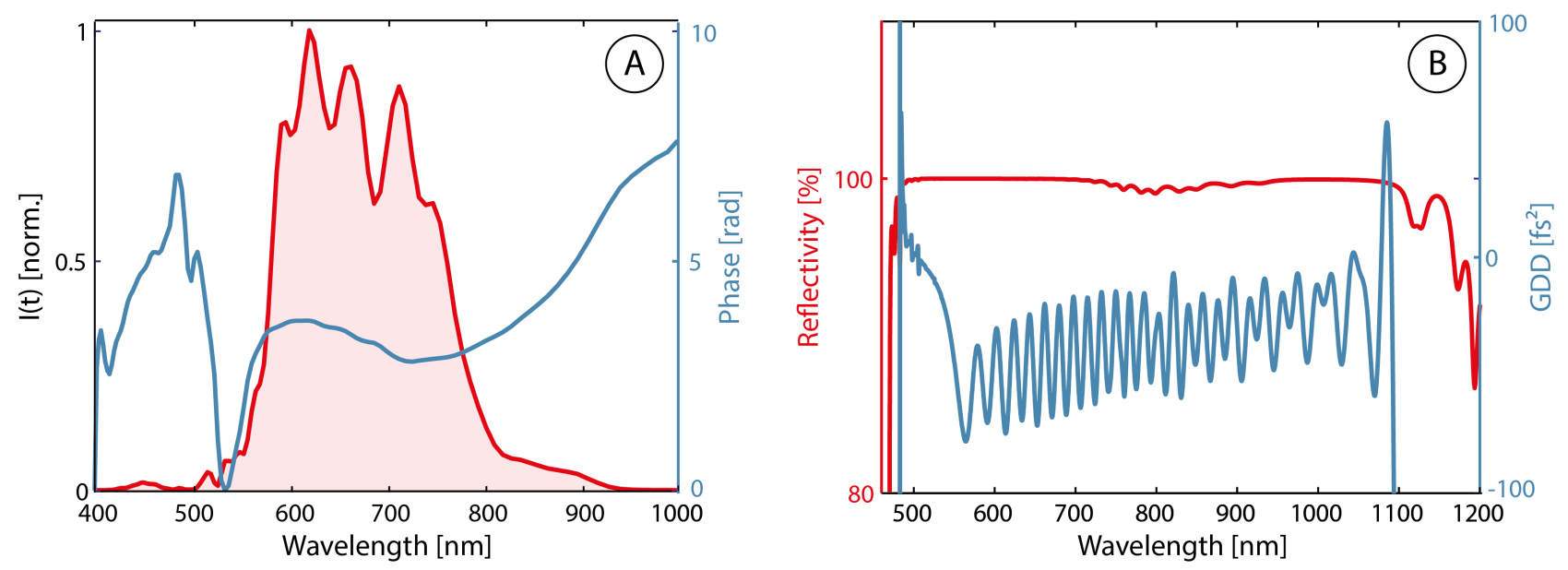

Figure 9.16: Panel A: The spectral intensity (red line) and phase (blue line) retrieved from the TG FROG spectrogram displayed in Fig.(9.15). Panel B: Design of the chirped multilayer mirrors to compress the laser pulse (appendix 9.2).

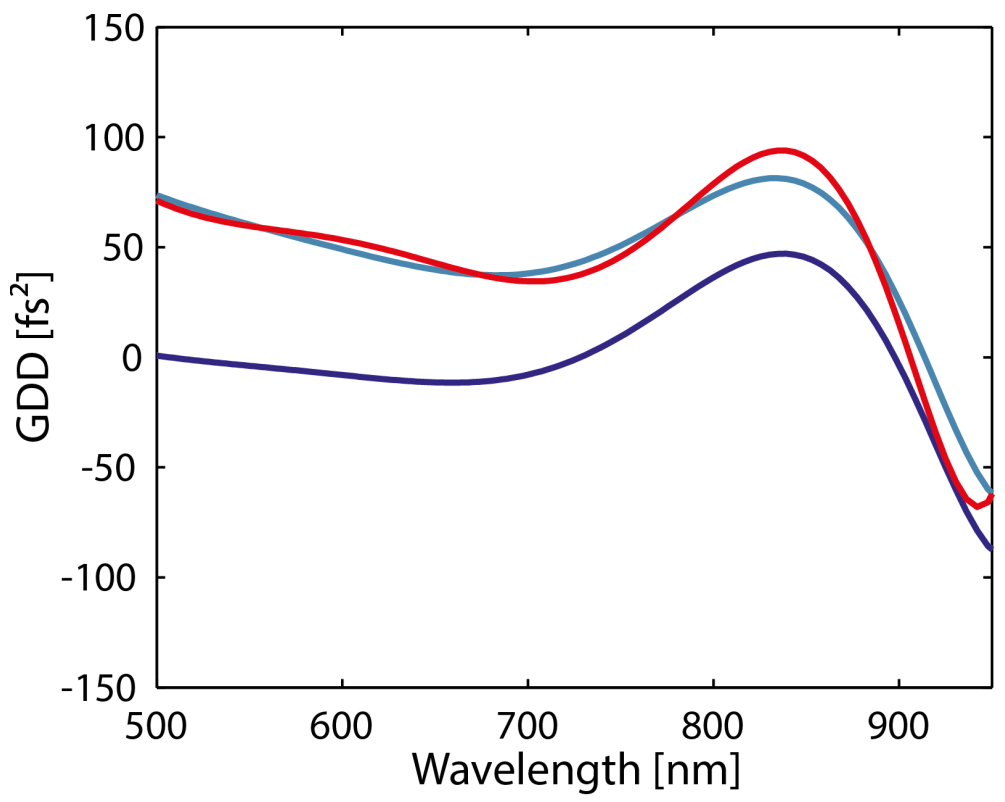

Figure 9.17: The group delay dispersion $G D D=\frac{\partial^{2} \varphi}{\partial \omega^{2}}$ is calculated from the phase information extracted with the TG FROG measurement (Fig.(9.16) (dark blue line). Theoretically the effect of $1 \mathrm{~mm}$ fused silica is added to the GDD curve (light blue line). The red line is retrieved from a TG FROG spectrogram recorded for the pulse after transmission through $1 \mathrm{~mm}$ additional glass. 


\subsection{Free Electron Trajectories in Neon Plasma}

Dr. Nicholas Karpowicz solved the time dependent Schroedinger equation together with the 1 dimensional wave equation to predict the plasma dynamics induced in Neon gas by the few-cycle waveform used in the experiment (section 6.3). The trajectories of the individual electrons are depicted in Fig.(9.18) together with the the average carrier motion $\langle x\rangle$ (dashed blue line). It is evaluated as the center of mass of the individual electron trajectories $\delta_{e^{-}}$. The prediction of the temporal evolution and amplitude of $\langle x\rangle$ coincides qualitatively and quantitatively with the results obtained experimentally with Eqn.(2.49).

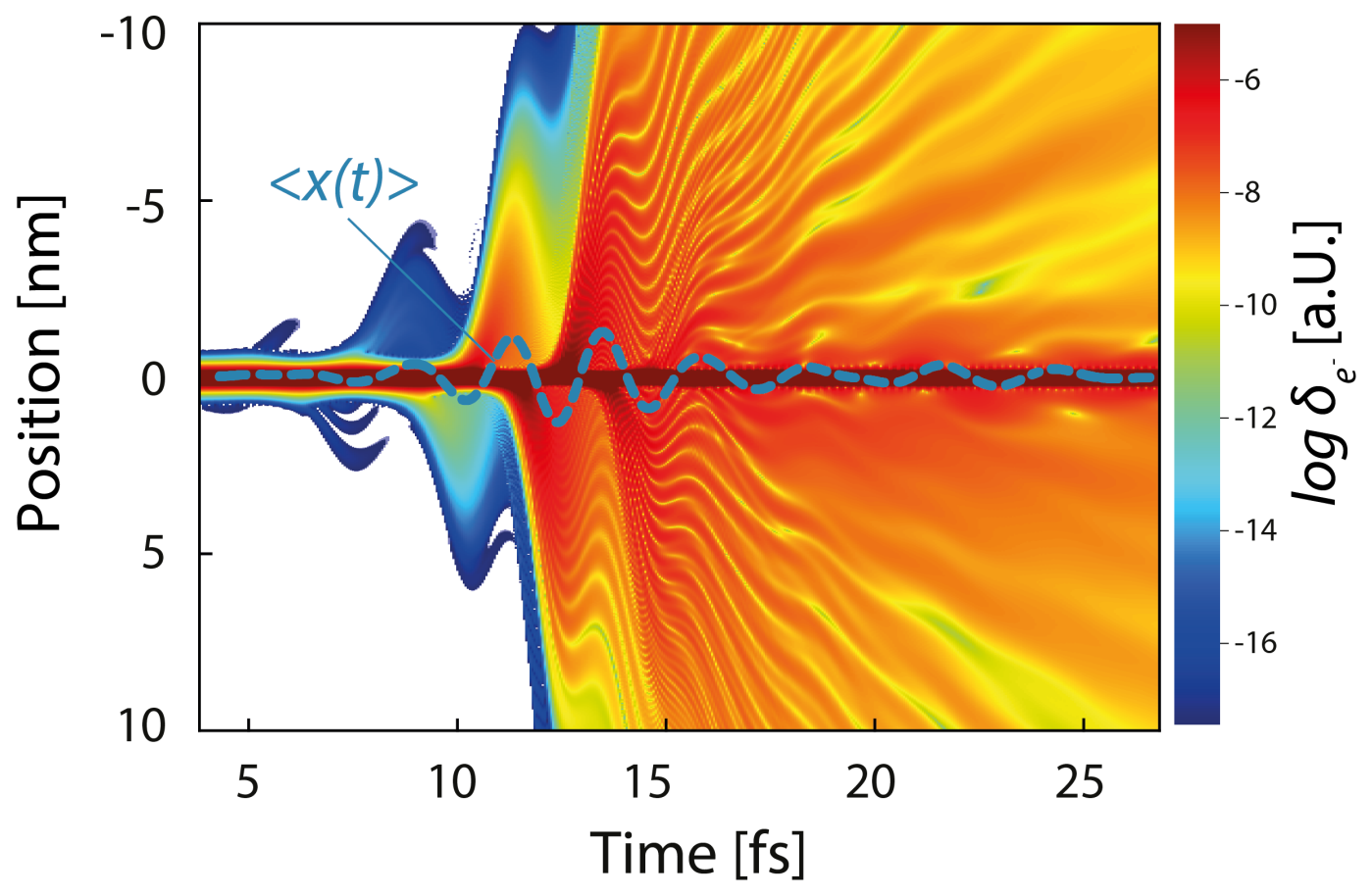

Figure 9.18: The theoretical prediction of the ensemble average position of the electron wavepacket (blue dashed line) is evaluated as the center of mass of the individual trajectories $\left(\delta_{e^{-}}\right)$.

\subsection{Damage Threshold Measurement}

In the course of this thesis the nonlinear interaction of insulators and few-cycle waves up to field strength close to optical breakdown is investigated. So far the exact mechanism behind optical damage induced by femtosecond laser pulses could not be entirely clarified [31]. The pulse durations are too short to cause considerable heat accumulation in the lattice which melts the medium [100]. To determine the exact boundary of optical breakdown for the pulses employed in this study (appendix 9.1.1) an independent damage threshold measurement is 
performed.

The sample is positioned in the focused laser beam under a small angle of incidence to monitor the beam profile of the light reflected from the front surface (Fig.(9.19)). Additionally the stray light from the sample excluding the direct reflection is collected by an large aperture lens. By stepwise moving the target towards the focus, the intensity it is exposed to is increased. At the moment of incipient damage the profile of the reflected beam is distorted and scattered light can be detected as displayed in Fig.(9.20). The irreversible material modifications are subsequently verified by ex situ examination of the targets under a light microscope.

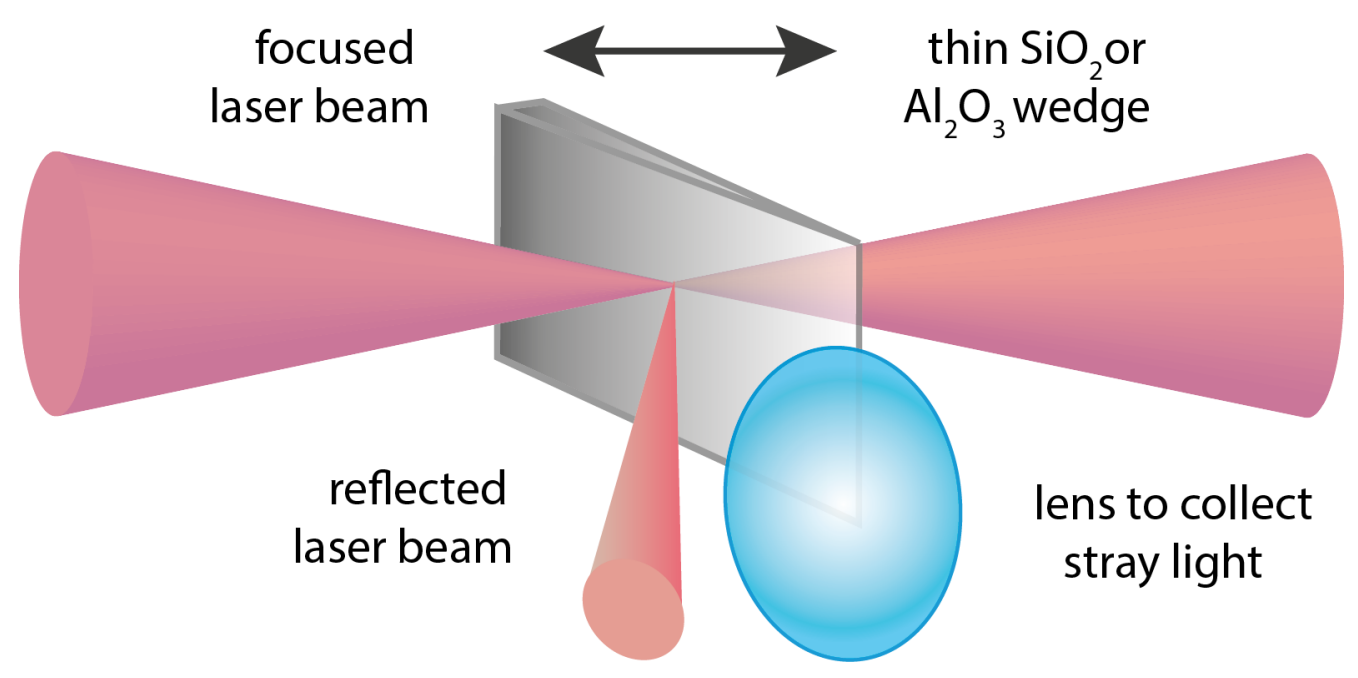

Figure 9.19: Schematic setup for the determination of the material damage threshold.
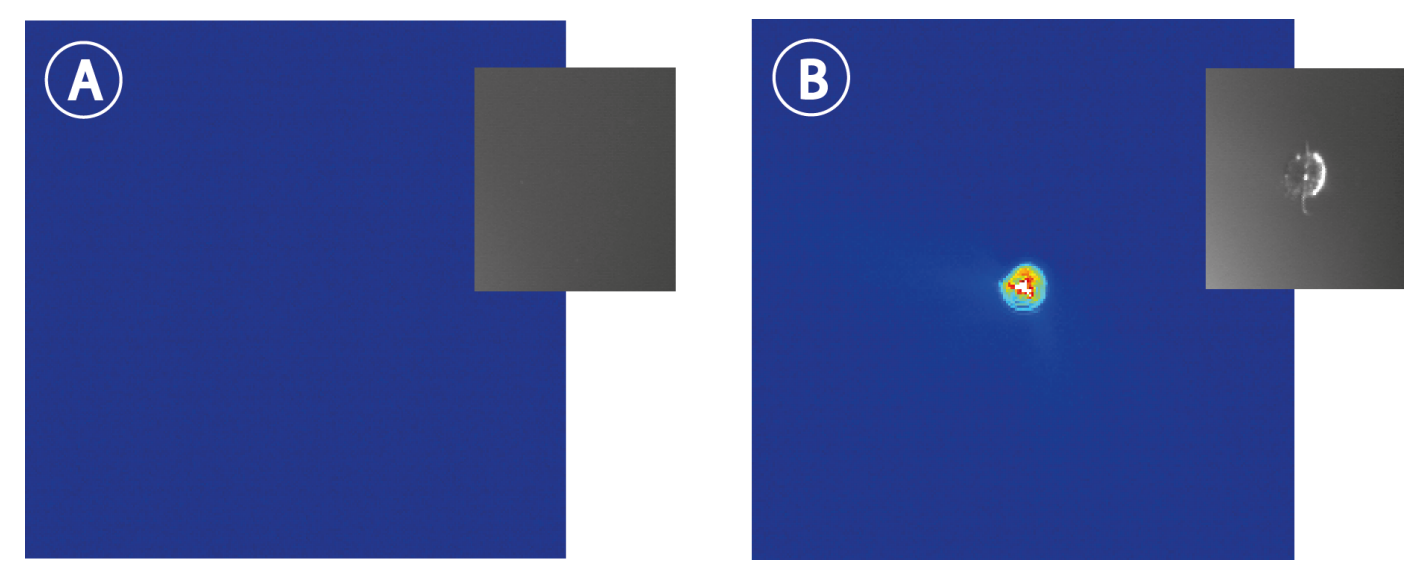

Figure 9.20: Panel A: The medium is exposed to intensities below the damage threshold where no light is scattered from the sound surface. Before performing the experiment a microscope picture of the surface is recorded (right). B: The damaged sample surface scatters an considerable amount of stray light. The hole ablated by the laser pulse is clearly visible under the microscope (right). 
To reliably distinguish damages on the front and back surface of the medium, in a first step wedged fused silica and sapphire samples (with an opening angle of $3^{\circ}$ ) are investigated. The front surface of the fused silica wedges is irreversible modified at an intensity of $I_{\mathrm{SiO}_{2} \text { wedge }}=(2.2 \pm 0.4) \cdot 10^{14} \frac{\mathrm{W}}{\mathrm{cm}^{2}}$ and sapphire at $I_{A l_{2} \mathrm{O}_{3} \text { wedge }}=(1.9 \pm 0.3) \cdot 10^{14} \frac{\mathrm{W}}{\mathrm{cm}^{2}}$. The damage threshold for both materials coincides within the error bar as $\mathrm{SiO}_{2}$ and $\mathrm{Al}_{2} \mathrm{O}_{3}$ possess a band gap of similar size $\left(\Delta E_{g \mathrm{Al}_{2} \mathrm{O}_{3}} \approx 8.8 \mathrm{eV}, \Delta E_{\mathrm{gSiO}} \approx 9 \mathrm{eV}\right)$. Additionally the intensity of optical breakdown for $50 \mu \mathrm{m}$ thick amorphous $\mathrm{SiO}_{2}$ and $\mathrm{Al}_{2} \mathrm{O}_{3}$ plates is determined to $I_{\mathrm{SiO}_{2} 50 \mu \mathrm{m}}=(2.0 \pm 0.2) \cdot 10^{14} \frac{\mathrm{W}}{\mathrm{cm}^{2}}$ and $I_{\mathrm{Al}_{2} \mathrm{O}_{3} 50 \mu \mathrm{m}}=(1.7 \pm 0.2) \cdot 10^{14} \frac{\mathrm{W}}{\mathrm{cm}^{2}}$. Due to the difficult manufacturing process of dielectric plates with $\mu \mathrm{m}$ thicknesses their optical surface quality is slightly inferior to the thicker wedges. Therefore they tend to be break at lower intensities.

\subsection{The Impact of Linear Material Dispersion on the Nonlin- earity}

As explained in section 6.4.1 the temporal evolution of the phase difference between $E_{r e f}(\ell, t)$ and $E(\ell, t)$ cannot be described by Kerr theory. Even though $\Delta \phi_{N L}$ peak rises linearly with the peak intensity of the driving field, the temporal profile of $\Delta \phi_{N L}(t)$ is not proportional to its intensity envelope (Fig.(6.11)). This discrepancy can be explained by the influence of dispersion on ultrashort broadband light pulses. The nonlinear Kerr response of the medium is confined to a time window which is approximately $1 / \sqrt{3}$ the duration of the fundamental pulse. Therefore the nonlinear polarization is affected to a higher degree by material dispersion during propagation than the driving field. New low frequency components generated in the interaction tend to outrun the underlying fundamental, while high frequencies are delayed. If field components emerging from the nonlinear process appear at times where the intensity of the linear propagated reference is close to zero, the phase is very sensitive to changes. In the experiment this is visible as pedestals near the nodes of the intensity envelope. Additionally it explains the discontinuity $\Delta \phi_{N L}(t=21 f s)$ at the local minimum of the field envelope (Fig.(6.11)) which is a numerical artifact and has no physical meaning.

To test the validity of the given explanation the pulse propagation through $10 \mu \mathrm{m}$ fused silica is numerically simulated by Dr. Nicholas Karpowicz with and without consideration of linear dispersion. He solves the one dimensional wave equation under assumption of the slowly varying wave approximation (section 2). The intensity of the strong field is adjusted to $I=5 \cdot 10^{13} \frac{\mathrm{W}}{\mathrm{cm}^{2}}$, the weak reference is assumed with $I_{\text {ref }}=1 \cdot 10^{11} \frac{\mathrm{W}}{\mathrm{cm}^{2}}$.

For the non-dispersive medium $\Delta \phi_{N L}(t)$ (Fig. (9.21) A, light blue line) the phase shift follows the intensity envelope of the driving field according to Kerr theory. The impact of self steepening is visible as the center of mass of the strong field envelope is delayed compared to the reference. Additionally the slope of the pulse is increased at its back and reduced at its front (section 2.3). In the dispersive material $\Delta \phi_{N L}(t)$ exhibits a spiky structure close to the nodes of the intensity envelope (Fig. (9.21) B). The phase of the reference field is almost zero 
at these points. Small changes in the phase of the strong field significantly affect $\Delta \phi_{N L}(t)$. In the simulation this modification is visible as spikes, in the experiment it induces pedestals. Due to dispersion also the impact of self-steepening is less pronounced.
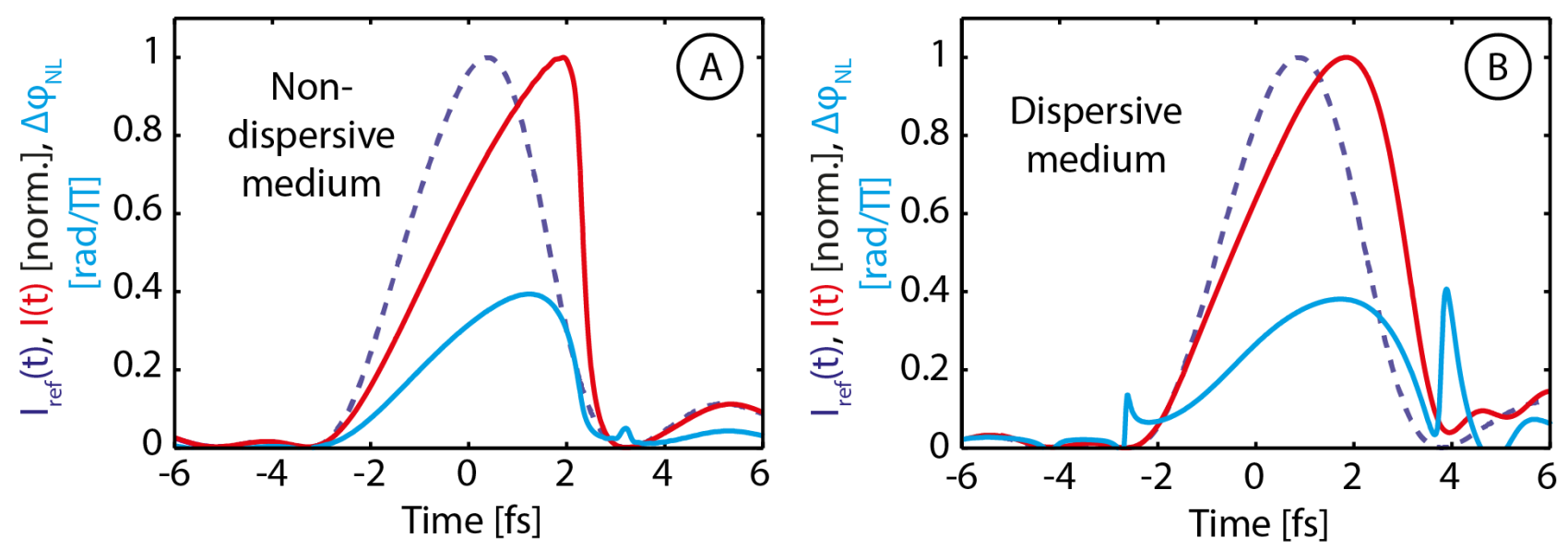

Figure 9.21: The intensity envelope of the reference (dark blue dashed line) and strong (red line) field after the theoretical propagation through $10 \mu \mathrm{m}$ fused silica. The phase difference between the two field is marked as the light blue line. Panel A shows the results obtained if material dispersion is ignored. In Panel B it is considered.

\subsection{Technical Implementation of the Wave Form Synthesizer}

The schematic setup of the wave form synthesizer is depicted in Fig. (9.22). The broadband spectrum of the few-cycle pulse (appendix 9.1.2) hits a beamsplitter which reflects wavelength from $400-600 \mathrm{~nm}$ (blue channel) and transmits the spectral range from $600-1100 \mathrm{~nm}$ (red channel Fig.(9.23 A)). Like this approximately $20 \%$ of the pulse energy are distributed to the blue and $80 \%$ to the red channel. The spectral reflectivity and transmittance of the beamsplitter are depicted in Fig.(9.24). The phase it introduces is designed as a constant with a negligible frequency dependence (section 9.5).

In both arms the beam is reflected off 6 pairs of negatively chirped multilayer mirrors (appendix 9.2) (in Fig. (9.22) only 3 pairs are displayed) individually designed and fabricated by Olga Razskazovskaya (MPQ) to compress the pulses in both arms (section 7.1, 7.1). For flexible dispersion control movable fused silica wedges are located in both channels. To minimize reflection losses they are positioned in Brewster's angle. Convex mirrors with slightly modifiable radius of curvature enable the variable compensation of small deviations in the beam divergence in both arms. To regulate the temporal overlap of the pulses and synthesize distinct waveforms a piezo stage is positioned in the blue channel. It additionally is part of an active delay stabilization between the two arms. After recombining the two channels with another beamsplitter (Fig.(9.24)) the pulse is transmitted through a glass window under Brewster's angle into the vacuum system. As the beamsplitter does not 


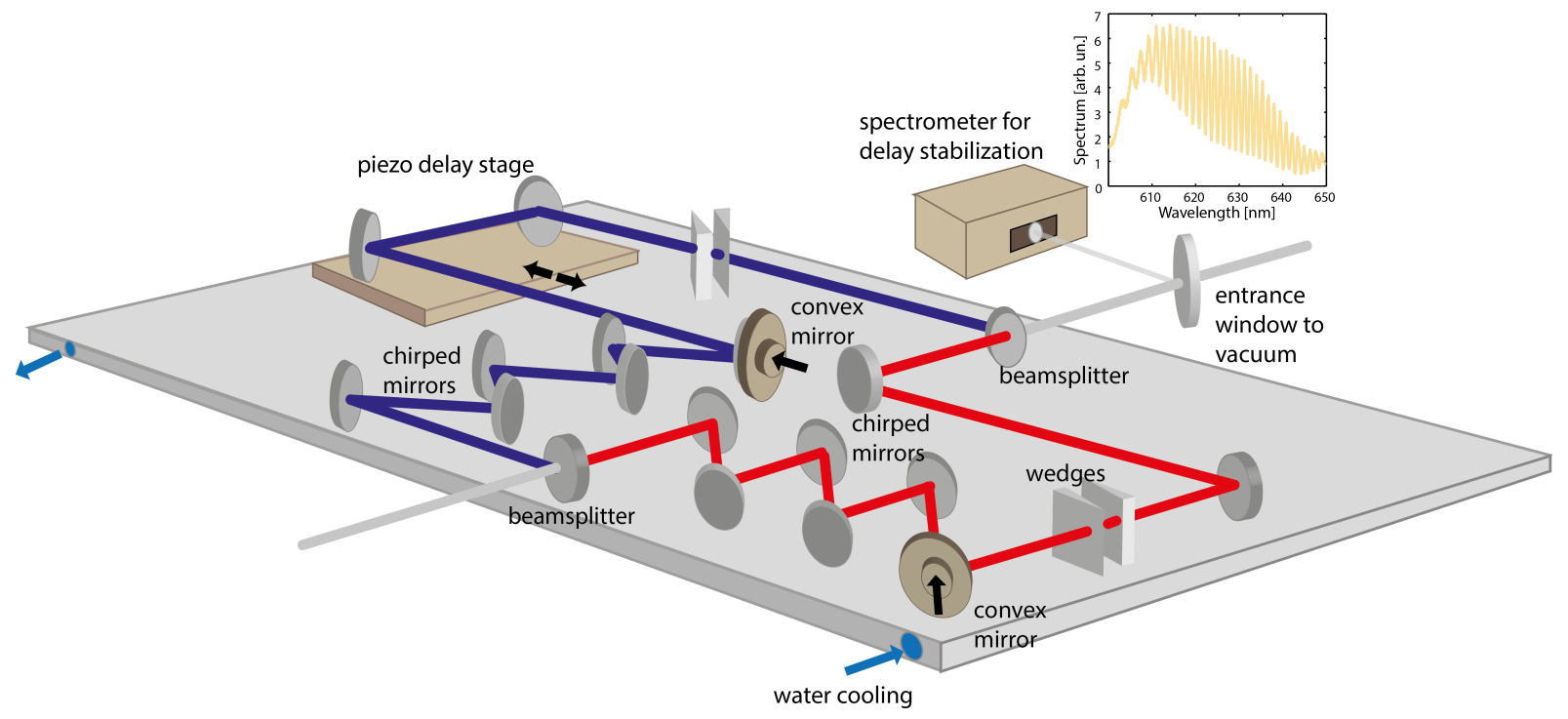

Figure 9.22: Schematic setup of the two channel wave form synthesizer.

perfectly divide the spectrum at $600 \mathrm{~nm}$, the blue channel still possess components around $600-650 \mathrm{~nm}$ (Fig.(9.23) A). These spectral components are reflected but not compressed by the chirped mirrors in the blue arm. As mentioned above the red channel is transmitted through the beamsplitter whereas the blue arm is reflected. The chirped mirrors are designed to compensate this difference in optical path length (appendix 9.2). As they do not work for the wavelength residues above $600 \mathrm{~nm}$ in the blue channel these components do not temporally overlap with the main blue pulse. When the main pulses out of the two arms are spatially and temporally recombined the beating is well visible in the wavelength range between $600-650 \mathrm{~nm}$ (Fig.(9.23) B). This interference structure can be tracked in the loss light reflected from the entrance window and utilized as an error signal for the active stabilization. The stabilization controls the optical path length of the blue arm by adjusting the piezo position. Like this the temporal jitter between the two channels caused by thermal drifts or slow mechanical vibrations can be compensated. 

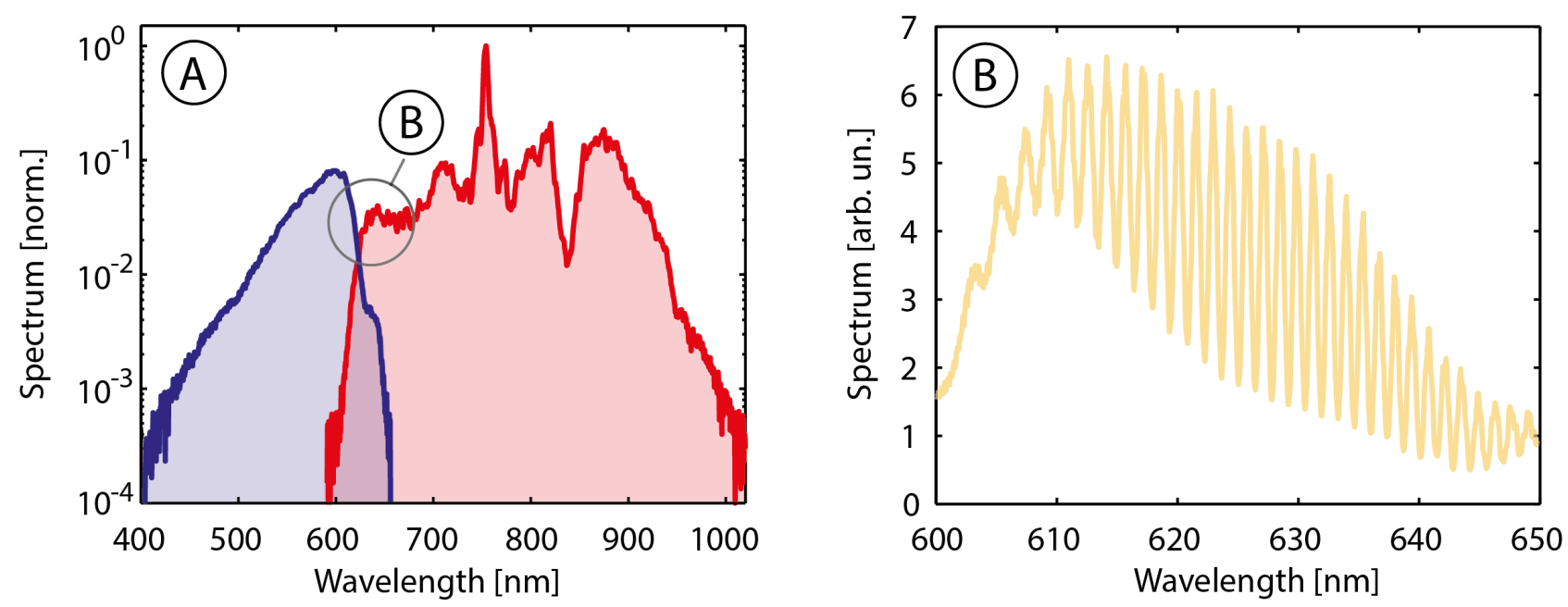

Figure 9.23: Panel A: The spectrum of the blue (blue line) and red channel (red line) of the wave form synthesizer. Panel B zooms into the spectral region between $600-650 \mathrm{~nm}$. Panel $\mathrm{B}$ : The interference pattern between the wavelength from $600-650 \mathrm{~nm}$ of the red arm and the residual components in the blue channel is used as a signal for the active delay stabilization.

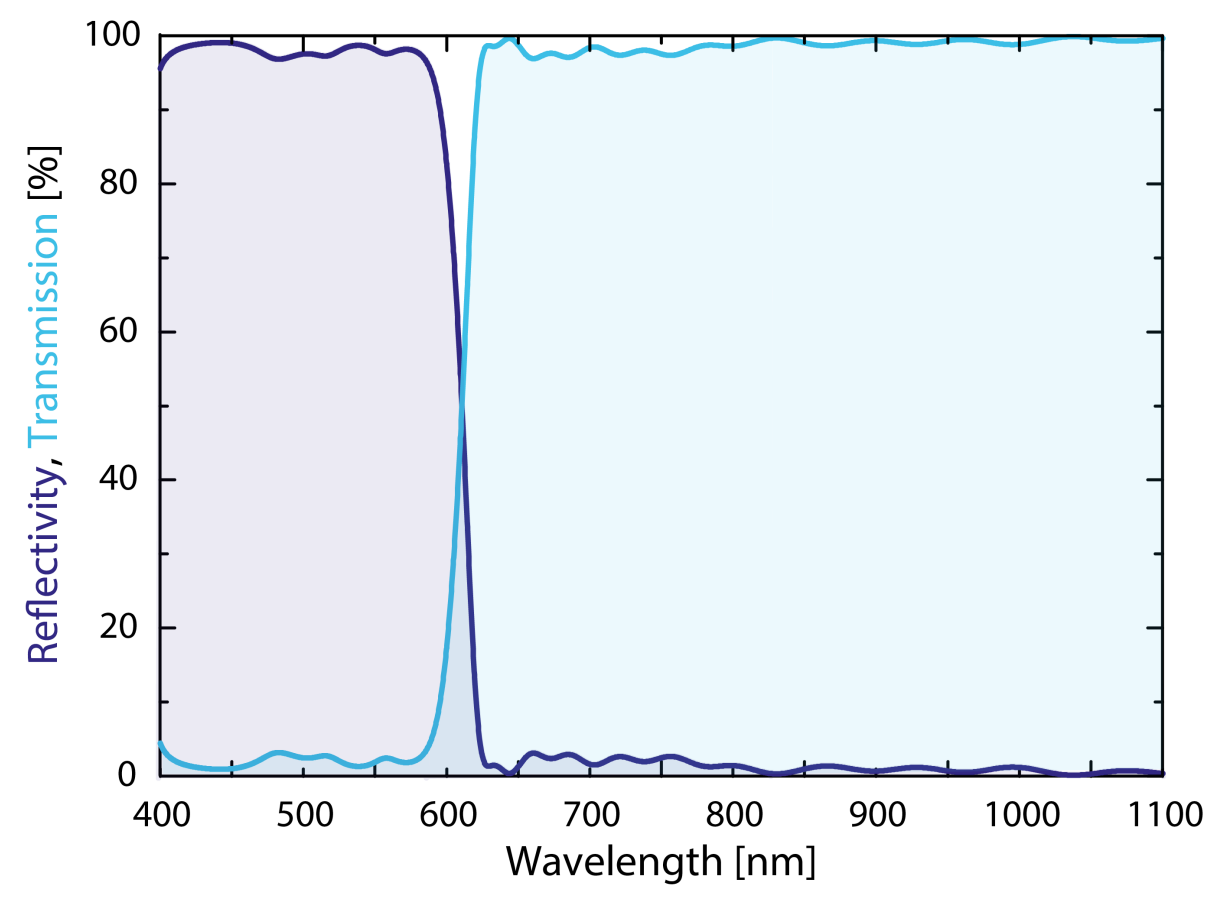

Figure 9.24: Reflectivity (dark blue line) and transmittance (light blue line) of the beamsplitter dividing the spectrum at $600 \mathrm{~nm}$ (fabricated by Olga Razskazovskaya, MPQ) 


\section{Chapter 10}

\section{Data Archiving}

All figures, raw data and evaluation software analyzed and developed in the course of this thesis is stored on the data archive computer of the Max Planck Institute for Quantum Optics, Division Attosecond Physics (Prof. Dr. Krausz). The following table of contents presents how the data is organized.

\begin{tabular}{|c|c|}
\hline \multicolumn{2}{|l|}{ chapter 1} \\
\hline Fig. 1.1 & schematic sketch (.png, .ai) \\
\hline \multicolumn{2}{|l|}{ chapter 2} \\
\hline Fig. 2.1 & simulation code (.m), schematic sketch (.png, .ai) \\
\hline Fig. 2.2 & schematic sketch (.png, .ai) \\
\hline Fig. 2.3 & schematic sketch (.png, .ai) \\
\hline Fig. 2.4 & schematic sketch (.png, .ai) \\
\hline Fig. 2.5 & schematic sketch (.png, .ai) \\
\hline \multicolumn{2}{|l|}{ chapter 3} \\
\hline \multicolumn{2}{|l|}{ chapter 3.1} \\
\hline Fig. 3.1 & schematic sketch (.png, .ai) \\
\hline Fig. 3.2 & schematic sketch (.png, .ai) \\
\hline fused silica & Fig. 3.3 (.png, .ai), raw data, evaluation code (.m) \\
\hline sapphire & Fig. 3.4 (.png, .ai), raw data, evaluation code (.m) \\
\hline \multicolumn{2}{|l|}{ chapter 3.2} \\
\hline Fig. 3.5 & schematic sketch (.png, .ai) \\
\hline fused silica & Fig. 3.6 (.png, .ai), raw data, evaluation code (.m) \\
\hline sapphire & Fig. 3.7 (.png, .ai), raw data, evaluation code (.m) \\
\hline \multicolumn{2}{|l|}{ chapter 4} \\
\hline Fig. 4.1 & schematic sketch (.png, .ai) \\
\hline Fig. 4.2 & schematic sketch, processed data (.png, .ai) \\
\hline Fig. 4.3 & processed data (.png, .ai) \\
\hline
\end{tabular}




\begin{tabular}{|c|c|}
\hline \multicolumn{2}{|l|}{ chapter 5} \\
\hline Fig. 5.1 & schematic sketch (.png, .ai) \\
\hline Fig. 5.2 & processed data (.png, .ai) \\
\hline Fig. 5.3 & schematic sketch, processed data (.png, .ai) \\
\hline Fig. 5.4 & processed data (.png, .ai) \\
\hline \multicolumn{2}{|l|}{ chapter 6} \\
\hline \multicolumn{2}{|l|}{ chapter 6.1} \\
\hline Fig. 6.1 & schematic sketch (.png, .ai) \\
\hline Fig. 6.2 & schematic sketch (.png, .ai) \\
\hline \multicolumn{2}{|l|}{ chapter 6.2} \\
\hline Fig. 6.3 & schematic sketch (.png, .ai) \\
\hline Fig. 6.4 & processed data (.png, .ai), raw data, evaluation code (.m) \\
\hline \multicolumn{2}{|l|}{ chapter 6.3} \\
\hline Fig. 6.5 & processed data (.png, .ai), raw data, evaluation code (.m) \\
\hline Fig. 6.6-6.8 & processed data (.png, .ai), raw data, evaluation code (.m) \\
\hline \multicolumn{2}{|l|}{ chapter 6.4} \\
\hline \multicolumn{2}{|l|}{ chapter 6.4 .1} \\
\hline Fig. 6.9-6.116.14 & processed data (.png, .ai), raw data, evaluation code (.m) \\
\hline Fig. 6.12-6.13 & processed data (.png, .ai), raw data, evaluation code (.m) \\
\hline \multicolumn{2}{|l|}{ chapter 6.4 .2} \\
\hline Fig. 6.15 & processed data (.png, .ai), raw data, evaluation code (.m) \\
\hline Fig. 6.16 & processed data (.png, .ai), raw data, evaluation code (.m) \\
\hline Fig. 6.17 & processed data (.png, .ai), raw data, evaluation code (.m) \\
\hline \multicolumn{2}{|l|}{ chapter 6.4 .3} \\
\hline Fig. 6.18 & processed data (.png, .ai), raw data, evaluation code (.m) \\
\hline Fig. 6.19 & processed data (.png, .ai), raw data, evaluation code (.m) \\
\hline Fig. 6.20 & processed data (.png, .ai), raw data, evaluation code (.m) \\
\hline \multicolumn{2}{|l|}{ chapter 6.4 .4} \\
\hline Fig. 6.21-23 & processed data (.png, .ai), raw data, evaluation code (.m) \\
\hline Fig. 6.24-25 & processed data (.png, .ai), raw data, evaluation code (.m) \\
\hline \multicolumn{2}{|l|}{ chapter 6.4 .6} \\
\hline Fig. 6.26-28 & processed data (.png, .ai), raw data, evaluation code (.m) \\
\hline \multicolumn{2}{|l|}{ chapter 7} \\
\hline Fig. 7.1 & processed data (.png, .ai), raw data, evaluation code (.m) \\
\hline Fig. 7.2-7.3 & processed data (.png, .ai), raw data, evaluation code (.m) \\
\hline Fig. 7.6 & processed data (.png, .ai), raw data, evaluation code (.m) \\
\hline \multicolumn{2}{|l|}{ chapter 9} \\
\hline \multicolumn{2}{|l|}{ chapter 9.1} \\
\hline Fig. 9.1 & processed data (.png, .ai), raw data, evaluation code (.m) \\
\hline \multicolumn{2}{|l|}{ chapter 9.2} \\
\hline Fig. 9.2 & schematic sketch (.png, .ai) \\
\hline Fig. 9.3 & schematic sketch (.png, .ai) \\
\hline
\end{tabular}




\begin{tabular}{|ll|}
\hline chapter 9.3 & \\
\hline Fig. 9.4 & schematic sketch (.png, .ai) \\
Fig. 9.5 & schematic sketch (.png, .ai) \\
Fig. 9.6 & schematic sketch (.png, .ai) \\
Fig. 9.7 & processed data (.png, .ai), raw data \\
\hline chapter 9.4 & \\
\hline Fig. 9.8 & schematic sketch (.png, .ai) \\
Fig. 9.9 & schematic sketch (.png, .ai) \\
Fig. 9.10 & schematic sketch (.png, .ai) \\
Fig. 9.11 & processed data (.png, .ai), raw data, evaluation code $(. m)$ \\
\hline chapter 9.5 & \\
\hline Fig. 9.12 & schematic sketch (.png, .ai) \\
Fig. 9.13 & schematic sketch (.png, .ai), simulated data, evaluation code $(. m)$ \\
Fig. 9.14 & schematic sketch (.png, .ai), simulation code (.m) \\
Fig. 9.15-9.16A & processed data (.png, .ai), raw data, evaluation code $(. m)$ \\
Fig. 9.16B & processed data (.png, .ai), evaluation code $(. m)$ \\
Fig. 9.17 & processed data (.png, .ai) \\
\hline chapter 9.6 & \\
\hline Fig. 9.18 & processed data (.png, .ai), raw data \\
\hline chapter 9.7 & \\
\hline Fig. 9.19 & schematic sketch (.png, .ai) \\
Fig. 9.20 & processed data (.png, .ai) \\
\hline chapter 9.8 & \\
\hline Fig. 9.21 & processed data (.png, .ai), raw data \\
\hline chapter 9.9 & \\
\hline Fig. 9.22 & processed data (.png, .ai), raw data, evaluation code $(. m)$ \\
Fig. 9.23 & \\
Fig. 9.24 & \\
\hline
\end{tabular}




\section{Bibliography}

[1] T. BRABEC, F. KRAUSZ: Intense few-cycle laser fields: Frontiers of nonlinear optics. In: Rev. Mod. Phys. 72 (2000), S. 545-591

[2] H. J. CAulfield, S. DOlEv: Why future supercomputing requires optics. In: Nature Photon. 4 (2010), S. 261-263

[3] D. Milam: Review and Assessment of Measured Values of the Nonlinear RefractiveIndex Coefficient of Fused Silica. In: Appl. Opt. 37 (1998), S. 546

[4] E. Yablonovitch et al: Virtual Photoconductivity. In: Phys. Rev. Lett. 63(9) (1989), S. 976-979

[5] Y. TAUR, T. H. NiNG: Fundamentals of Modern VLSI devices. 2nd edition. Cambridge : Cambridge Universtity Press, 2009

[6] WIKIPEDIA: Moore's law. - https://en.wikipedia.org/wiki/Moore

[7] D. P. SANDERS: Advances in Patterning Materials for $193 \mathrm{~nm}$ Immersion Lithography. In: Chemical Reviews 110 (2010), Nr. 1, S. 321-360

[8] IBM: IBM Z13. - http://www-03.ibm.com/systems/de/z/hardware/z13.html

[9] R. C. JOHNSON: IBM Leapfrogs Intel to 7nm. In: EETimes 7(9) (2015)

[10] D. KAHNG: Electric field controlled semiconductor device. In: US patent 3102230 (1963)

[11] J. J. Liou, F. SChWierz: Modern Microwave Transistors: Theory, Design and Performance. In: Wiley-Interscience (2003)

[12] J. J. LIOU, F. SCHWIERZ: RF transistors: recent developments and roadmap toward terahertz applications. In: Solid-State Electron. 51 (2007), S. 1079-1091

[13] W. R. DeAl ET AL.: Low noise amplification at 0.67THz using 30nm InP HEMTs. In: IEEE Microw. Wirel. Compon. Lett. 21 (2011), S. 368-370

[14] D. MamaluY, X. GaO: The fundamental downscaling limit of field effect transistors. In: Applied Physics Letters 106 (2015), Nr. 19, S. 193503 
[15] R. Gross, A. MARx: Festkörperphysik. 2nd edition. München : de Gruyter Oldenbourg, 2014

[16] C. ZENER: Theory of the Electrical Breakdown of Solid Dielectrics. In: Royal Society of London Proceedings Series A 145 (1934), S. 523-529

[17] G. WANNIER: Wave Functions and Effective Hamiltonian for Bloch Electrons in an Electric Field. In: Physical Review 117 (1960), S. 432-439

[18] W. FRANZ: Einfluss eines elektrischen Feldes auf eine optische Absorptionskante. In: Zeitschrift Naturforschung Teil A 13 (1958), S. 484

[19] L. V. Keldysh: Behavior of Non-metallic Crystals in Strong Electric Fields. In: Soviet Journal of Experimental and Theoretical Physics 6 (1958), S. 763

[20] R. Atanasov, A. Hachee, H. M. vam Driel, J. E. Sipe: Coherent Control of Photocurrent Generation in Bulk Semicondurctors. In: Phys. Rev. Lett. 76(10) (1996), S. 1703-1706

[21] T. Nagatsuma: Photonic measurement technologies for high-speed electronics. In: Meas. Sci. Technol. 13 (2002), S. 1655-1663

[22] D. H. Auston: Picosecond optoelectronic switching and gating in silicon. In: Appl. Phys. Lett. 26 (1975), S. 101-103

[23] D. H. Auston: Ultrafast optoelectronics. In: Top. Appl. Phys. 60 (1988), S. 183-233

[24] H. Shimosato, M. Ashida,T. ItOH, S. SAito, K. SAKAi: Ultrabroadband Detection of Terahertz Radiation from 0.1 to $100 \mathrm{THz}$ with Photoconductive Antenna. In: Ultrafast Optics V, Springer Series in Optical Sciences Volume 132

[25] R. Huber, A. A. Anappara, G. Günter, A. Sell, S. De liberato, C. Ciuti, G. BiAsiol, L. SORBA, A. TREDICUCCI, A. LeITENSTORFER: Switching ultrastrong lightmatter coupling on a subcycle scale. In: Journal of Applied Physics 109(10) (2011), S. 102418

[26] N. S. Pate, K. L. Hall, K. A. Rauschenbach: Interferometric all-optical switches for ultrafast signal processing. In: Appl. Opt. 37(14) (1998), S. 2831-2842

[27] R. Ulbricht, E. Hendry, J. Shan, T. F. Heinz, M. Bonn: Carrier dynamics in semiconductors studied with time-resolved terahertz spectroscopy. In: Rev. Mod. Phys. 83 (2011), S. 543-586

[28] W. KUeHn ET AL.: Terahertz-induced interband tunneling of electrons in GaAs. In: Phys. Rev. B 82 (2010), S. 075204 
[29] A. Schiffrin, T. PaAsch-Colberg, N. Karpowicz, V. Apalkov, D. Gerster, S. MÜhlbrandt, M. L. Korbman, J. Reichert, M. Schultze, S. Holzner, J. V. Barth, R. Kienberger, R. ERnstorfer, V. S. YAKOVleV, M. I. StOckman, F. KRAUSZ: Optical-field-induced current in dielectrics. In: Nature 493 (2012), S. 70-74

[30] A. P. Pati, I. S. Wahyutama, A. N. Pfeiffer: Subcycle-resolved probe retardation in strong-field pumped dielectrics. In: Nat. Commun. 6 (2015), S. 7746

[31] M. LenZER: Femtosecond Induced Laser-Induced Damage of Dielectrics. In: International Journal of Modern Physics B 13 (1999), Nr. 13, S. 1559-1578

[32] M. Lenzner, J. Krüger, S. Sartania, Z. Cheng, Ch. Spielmann, G. Mourou, W. KAUteK, F. KRAUSZ: Femtosecond Optical Breakdown in Dielectrics. In: Phys. Rev. Lett. 80 (1998), S. 4076

[33] R. BoyD: Nonlinear Optics. 3rd edition. Burlington : Academic Press, 2008

[34] G. AgraWAL: Nonlinear Fiber Optics (Fourth Edition). 4th edition. San Diego : Academic Press, 2006

[35] T. Brabec, F. Krausz: Nonlinear Optical Pulse Propagation in the Single-Cycle Regime. In: Phys. Rev. Lett. 78 (1997), S. 3282-3285

[36] J.-C. Diels, R. WolfGANG : Ultrashort laser pulse phenomena. Academic press, 2006

[37] L. Gaeta, L. Alexander: Catastrophic Collapse of Ultrashort Pulses. In: Phys. Rev. Lett. 84 (2000), S. 3582-3585

[38] G. A. ReIDER: Photonik, eine Einführung in die Grundlagen. zweite, überarbeitete und erweiterte Auflage. Wien : Springer-Verlag, 2005

[39] M. GeISSLER ET AL.: Light Propagation in Field-Ionizing Media: Extreme Nonlinear Optics. In: Phys. Rev. Lett. 83 (1999), S. 2930-2933

[40] E. L. BuCKLAND, R. W. BOYD: Electrostrictive contribution to the intensity-dependent refractive index of optical fibers. In: Opt. Lett. 21 (1996), S. 1117

[41] A. J. TAylor, G. Rodriguez, T. S. Clement: Determination of $\mathrm{n} 2$ by direct measurement of the optical phase. In: Opt. Lett 21 (1996), S. 1812

[42] M. Sheik-Bahae, A. Said, T. Wei, D. J. Hagan, E. W. Van Stryland: Sensitive Measurement of Optical Nonlinearities Using a Single Beam. In: IEEE Journal of Quantum Electronics 26 (1990), S. 760

[43] A. Dementev, A. Jovaisa: Pulse Shape Influence on the Accuracy of Z-scan Measurements. In: Nonlinear Analysis: Modelling and Control 10 (2005), S. 119-136 
[44] F. A. Oguama, H. Garcia, A. M. Johnson: Simultaneous measurement of the Raman gain coefficient and the nonlinear refractive index of optical fibers: theory and experiment. In: J. Opt. Soc. Am. B. 22(2) (2005), S. 426

[45] K. F. RenK: Basics of Laser Physics: For Students of Science and Engineering. 1st edition. Springer Science Business Media, 2012

[46] J. N. Sweetser, D. N. Fittinghoff, R. Trebino: Transient-grating frequencyresolved optical gating. In: Optics Letters 22 (1997), S. 706-709

[47] M. Schultze, E. M. Bothschafter, A. SOMmer, S. Holzner, W. Schweinberger, M. Fiess, M. Hofstetter, R. Kienberger, V. Apalkov, V. S. Yakovlev, M. I. STOCKMAN, F. KRAUSZ: Controlling dielectrics with the electric field of light. In: Nature 493 (2012), S. 75-78

[48] V. Apalkov, M. I. StockmanN: Theory of Dielectric Nanofilms in Strong Ultrafast Optical Fields. In: Phys. Rev. B 86 (2012), S. 165118-1-13

[49] R. Huber ET AL.: Femtosecond Formation of Coupled Phonon-Plasmon Modes in InP: Ultrabroadband THz Experiment and Quantum Kinetic Theory. In: Phys. Rev. Lett. 94 (2005), S. 027401

[50] F. JUNGINGER ET AL.: Nonperturbative Interband Response of a Bulk InSb Semiconductor Driven Off Resonantly by Terahertz Electromagnetic Few-Cycle Pulses. In: Phys. Rev. Lett. 109 (2012), S. 6147403

[51] M. C. Hoffmann et Al.: Terahertz Kerr Effect. In: Appl. Phys. Lett. 95 (2009), S. 23

[52] M. CoRnet ET AL.: Terahertz Kerr effect in gallium phosphide crystal. In: J. Opt. Soc. Am. B. 31 (2014), S. 7

[53] M. ZalKovsKIJ ET AL.: Terahertz-induced Kerr effect in amorphous chalcogenide glasses. In: Appl. Phys. Lett. 103 (2013), S. 221102

[54] WIKIPEDIA: Michelson Interferometer. - https://de.wikipedia.org/wiki/MichelsonInterferomete

[55] J. Itatani, F. Quere, G. L. Yudin, M. Yu. Ivanov, F. Krausz, P. B. Corkum: Attosecond Streak Camera. In: Phys. Rev. Lett. 88(17) (2002), S. 173903

[56] V. S. POPOV: Tunnel and multiphoton ionization of atoms and ions in a strong laser field. In: Physics-Uspekhi 47(9) (2004), S. 855-885

[57] WIKIPEDIA: Ionization Energy. - https://en.wikipedia.org/wiki/Ionization energies of the elements

[58] WIKIPEDIA: Hilbert-Transformation. - https://de.wikipedia.org/wiki/HilbertTransformation 
[59] S. Wilks, J. Dawson, W. Mori, T. Katsouleas, M. Jones: Photon accelerator. In: Phys. Rev. Lett. 62 (1989), S. 2600-2603

[60] C. Bree, A. Demircan, G. Steinmeyer: Saturation of the All-Optical Kerr Effect. In: Phys. Rev. Lett 106 (2011), S. 183902

[61] C. KÖHLER ET AL.: Saturation of the nonlinear refractive index in atomic gases. In: Phys. Rev. A 87 (2013), S. 043811

[62] J. K. Wahlstrand, Y.-H. Cheng, H. M. Milchberg: High Field Optical Nonlinearity and the Kramers-Kronig Relations. In: Phys. Rev. Lett. 109 (2012), S. 113904

[63] V. Loriot, E. Hertz, O. FAUCher, B. LaVorel: Measurement of high order Kerr refractive index of major air components. In: Opt. Express 18 (2009), S. 13429

[64] M. V. Ammosov, N. B. Delone, V. P. Krainov: Tunnel ionization of complex atoms and of atomic ions in an alternating electromagnetic field. In: Zh. Eksp. Teor. Fiz. 91 (1986), S. 2008-2013

[65] A. Scrinzi, M. Geissler, T. Brabec: Ionization Above the Coulomb Barrier. In: Phys. Rev. Lett. 83 (1990), S. 706-709

[66] B. Borchers, C. Bree, S. Birkholz, A. Demircan, G. Steinmeyer: Saturation of the all-optical Kerr effect in solids. In: Opt. Lett 37 (2012), S. 1541â3

[67] V. Temnov et AL.: Multiphoton Ionization in Dielectrics: Comparison of Circular and Linear Polarization. In: Phys. Rev. Lett. 97 (2006), S. 237403

[68] M. YAMANishi: Field-Induced Optical Nonlinearity Due to Virtual Transitions in Semiconductor Quantum-Well Structures. In: Phys. Rev. Lett. 59(9) (1987), S. 1014-1017

[69] D. S. Chemla, D. A. B. Miller, S. Schmitt-RinK: Generation of Ultrashort Electrical Pulses through Screening by Vitrual Population in Biased Quantum Wells. In: Phys. Rev. Lett. 59(9) (1987), S. 1018-1021

[70] D. A. B. Miller: Are optical transistors the logical next step? In: Nature Photonics 4 (2010), S. 3-5

[71] L. B. KISH: End of Moore's law: thermal (noise) death of integration in micro and nano electronics. In: Phys. Lett. A 305 (2002), S. 144-149

[72] A. Lipson, S. G. Lipson, H. Lipson: Optical Physics. 4th edition. Cambridge : Cambridge University Press, 1998

[73] T. T. LuU, M. GarG, S. Y. Kruchinin, A. Moulet, M. T. Hassan, E. GoulielMAKIS: Extreme ultraviolet high-harmonic spectroscopy of solids. In: Nature 521 (2015), S. $498-502$ 
[74] R. TReBino: Frequency-resolved optical gating: The measurement of Ultrashort Laser Pulses. 1st edition. Kluwer Academic Publishers Group, 2002

[75] M. Schultze et Al.: Attosecond band-gap dynamics in silicon. In: Science 346 (2014), S. $1348-1352$

[76] D. Fabris, T. Witting, W. A. Okell, D. J. Walke, P. Matia-Hernando, J. Henkel, T. R. BARIllot, M. Lein, J. P. Marangos, J. W. G. Tisch: Synchronized pulses generated at $20 \mathrm{eV}$ and $90 \mathrm{eV}$ for attosecond pump-probe experiments. In: Nature Photonics 9 (2015), S. 383-387

[77] W. FRANZ: EinfluÃ eines elektrischen Feldes auf eine optische Absorptionskante. In: Z. Naturforschung 13a (1958), S. 484-489

[78] L. Cavalieri, E. Goulielmakis, B. Horvath, W. Helml, M. Schultze, M. Fiess, V. Pervak, L. Veisz, V. S. YaKovlev, M. Uiberacker, A. Apolonski, F. Krausz, R. KIENBERGER: Intense 1.5-cycle near infrared laser waveforms and their use for the generation of ultra-broadband soft-x-ray harmonic continua. In: New Journal of Physics 9 (2007), S. 242

[79] F. Krausz, M. E. Fermann, T. Brabec, P.r F. Curley, M. Hofer, M. H. Ober, C. SPIELMANN, E. WinTNER, A .J. SCHMIDT: Femtosecond Solid-State Lasers. In: IEEE Journal of Quantum electronics 28(10) (1992), S. 2097-2122

[80] M. FIESS: Advancing attosecond metrology, Diss., 2010

[81] R. Strickland, G. Mourou: Compression of amplified chirped optical pulses. In: Optics Communications 56(7) (1985), S. 219-221

[82] M. SCHULTZE: Attosecond real time observation of ionization and electron-electron interactions, Diss., 2008

[83] A. Baltuska, T. Udem, M. Uiberacker, M. Hentschel, E. Goulielmakis, Ch. GOHLE, R. HOlzWARTH, V. S. YAKOVleV, A. SCRINZI, T. W. HÄNSCH, F. KRAUSZ: Attosecond control of electronic processes by intense light fields. In: Nature 421 (2003), S. $611-615$

[84] W. Schweinberger, A. Sommer, E. M. Bothschafter, J. Li, F. Krausz, R. KienBERGER, M. SCHULTZE: Waveform-controlled near-single-cycle milli-joule laser pulses generate sub-10nm extreme ultraviolet continua. In: Optics Letters 37(17) (2012), S. 3573-3575

[85] D. N. Papadopoulos, I. Martial, M. Hanna, F. Druon, P. Georges : Active spectral phase control by use of an acousto-optic programmable filter in high-repetitionrate sub- 80fs nonlinear fiber amplifiers. In: Optics Letters 33(13) (2008), S. 1431-1433

[86] FASTLiTE: Dazzler. - http://www.fastlite.com/en/cat465015-Dazzler.html?Cookie=set 
[87] E. B. TREACY: Optical pulse compression with diffraction gratings. In: IEEE J. Quantum Electron 5 (1969), S. 454

[88] N. MatuscheK, F. X. KÄRTNeR, U. Keller, : Theory of Double-Chirped Mirrors. In: IEEE Journal of selected topics in quantum electronics, 4(2) (1998), S. 197

[89] F. X. Kärtner, N. Matuschek, T. Schibli, U. Keller, H. A. Haus, C. Heine, R. MORF, V. SCHEUER, M. TILSCH, T. TSCHUdi : Design and fabrication of doublechirped mirrors. In: Optics Letters 22(11) (1997), S. 831

[90] F. X. Kärtner, U. Morgner, R. Ell, T. Schibli, J. G. Fujimoto, E. P. Ippen,V. SCHEUER, G. ANGELOW, T. TSCHUDI : Ultrabroadband double-chirped mirror pairs for generation of octave spectra. In: J. Opt. Soc. Am. B 18(6) (2001), S. 882

[91] P. B. CORKUM: Plasma perspective on strong-filed multiphoton. In: Phys. Rev. Lett. 71(13) (1993), S. 1994-1997

[92] J. L. Krause, K. J. Schafer, K. C. Kulander: High-order harmonic generation from atoms and ions in the high intensity regime. In: Phys. Rev. Lett. 68(24) (1992), S. 3535-3538

[93] K. L. IsHIKAWA: High-Harmonic Generation. - Photon Science Center, Graduate School of Engineering, University of Tokyo, http://ishiken.free.fr/Publication/intech. pdf

[94] M. Lewenstein, P. Balcou, M. Y. Ivanov, A. L'Huillier, P. B. Corkum: Theory of high-harmonic generation by low-frequency laser fields. In: Phys. Rev. A 49(3) (1994), S. $2117-2132$

[95] M. Hentschel, R. Kienberger, C. Spielmann, G. A. Reider, N. Milosevic, T. Brabec, P. Corkum, U. Heinzmann, M. Drescher, F. Krausz: Attosecond metrology. In: Nature 414 (2001), S. 509

[96] J. Gagnon, V. S. Yakovlev: The direct evaluation of attosecond chirp from a streaking measurement. In: Appl. Phys. B 103 (2011), S. 303-309

[97] M. Hofstetter, M. Schultze, M. Fiess, B. Dennhardt, A. Guggenmos, J. Gagnon, V. S. YaKovlev, E. Goulielmakis, R. Kienberger, E. M. Gullikson, F. KRAUSZ, U. KleineberG: Attosecond dispersion control by extreme ultraviolet multilayer mirrors. In: Optics Express 19(3) (2011), S. 1767-1776

[98] M. HOFSTETTER: Reflectivity of silicon- molybdenum multilayer mirror $\left(A O I 5^{\circ}\right)$

[99] J. A. Shirley, R. J. HAll, A. C. ECKBRETH: Folded BOXCARS for rotational Raman studies. In: Optics letters 5(9) (1980), S. 380 
[100] United States. National Bureau of Standards American Society for TeSting AND Materials United States. OfFice of NAVAl Research United States. ENERGY RESEARCH AND DEVELOPMENT ADMINISTRATION: Laser induced damage in optical materials, 1975: proceedings of a symposium. U.S. Dept. of Commerce, National Bureau of Standards, 1976 


\section{Acknowledgement}

At first I would like to thank my PhD supervisor Prof. Dr. Ferenc Krausz for giving me the opportunity to be part of his group. It is an honor to work with such a skilled and experienced expert. He always has interesting ideas for new research and provides laboratories offering perfect conditions for experimental work. I want to thank him for his scientific and human advise.

The same is true for Dr. Martin Schultze who coordinates the project I work in. From my first day in the group he shared his extensive scientific and technical knowledge with me (with endless patience!) and taught me basically everything I know in the laboratory. Besides he endured me as an office mate and helped me in many personal matters.

I want to thank Dr. Elisabeth Bothschafter for a very effective teamwork and a fun time in the office and in the laboratory.

All my colleagues did their best to make work at MPQ pleasant and enjoyable. I want to thank Dr. Wolfgang Schweinberger, Clemens Jakubeit, Tobias Latka, Dr. Michael Jobst, Vahe Shirvanyan, Alexander Schwarz and Marcus Ossiander for their support at the weekend and in endless night shifts. Dr. Hanieh Fattahi and Dr. Nicholas Karpowicz always answered even the most stupid questions patiently and Olga Razskazovskaya coated uncountable pizza and non-pizza mirrors.

As the list of names of all people who helped me during the last years would be too long to fit on one page, I would like to thank all employees of the Krausz group and MPQ for the great working atmosphere and fun time.

Last but for sure not least I have to mention my parents who have always supported me in every possible way and Dr. Simon Holzner: I guess now both of us are officially 'nerds'! 